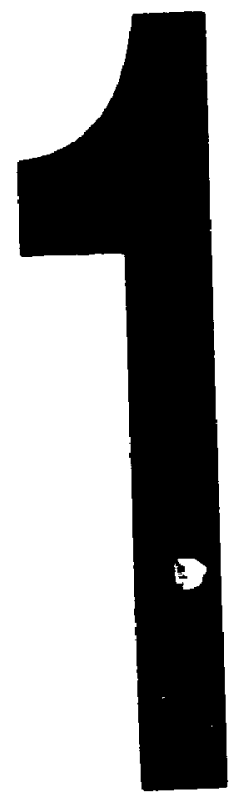

PM-1 31/2" 4 4" PHOTOGRAPHIC MICROCOPY TARGET NBS 1010 a ANSI/ISO \#2 EQUIVALENT

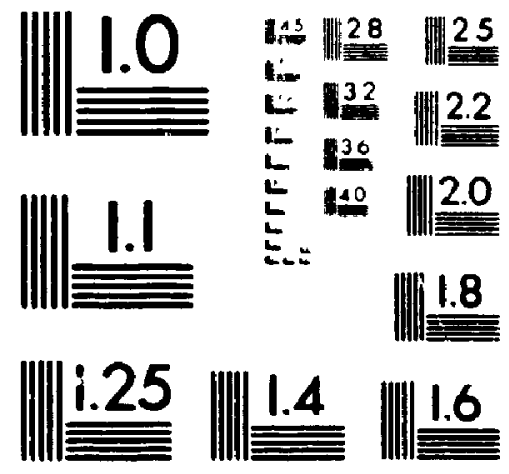

PRECISIONSM RESOLUTION TARGETS 
Nationai Litran

of Canada

Acquisitions and

Bibliographic Services Branch

395 Wellungton Street

Onawa. Ontarno

KIA ON4
Biblıothèque natconale

du Canada

Direction des acquisitions et des services bibliographiques

395. ne Wielhnglon

Ottawa (Ontarno)

KIA ONA
NOTICE

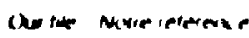

AVIS
The quality of this microform is heavily dependent upon the quality of the original thesis submitted for microfilming. Every effort has been made to ensure the highest quality of reproduction possible.

If pages are missing, contact the university which granted the degree.

Some pages may have indistinct print especially if the original pages were typed with a poor typewriter ribbon or if the university sent us an inferior photocopy.
La qualité de cette microforme dépend grandement de la qualité de la thès: soumise au microfilmage. Nous avons tout fait pour assurer une qualité supérieure de reproduction.
S'il manque des pages, veuillez communiquer avec l'université qui a conféré le grade.

La qualité d'impression de certaines pages peut laisser à désirer, surtout si les pages originales ont été dactylographiées à l'aide d'un ruban usé ou si l'université nous a fait parvenir une photocopie de qualité inférieure.

La reproduction, même partielle, de cette microforme est soumise à la Loi canadienne sur le droit d'auteur, SRC 1970, c. C-30, et ses amendements subséquents.
Reproduction in full or in part of this microform is governed by the Canadian Copyright Act, R.S.C. 1970, C. C-30, and subsequent amendments. 


\title{
A High Order Simulation Model for the Bell 205 Helicopter
}

\author{
by \\ Abdol-Reza Neshat. B.Sc. \\ A thesis submitted to \\ the Faculty of Graduate Studies and Research \\ in partial fulfilment of \\ the requirements for the degree of \\ Master of Engineering \\ Ottawa-Carleton Institute for \\ Mechanical and Aerospace Engineering
}

\author{
Department of \\ Mechanical and Aerospace Engineering \\ Carleton University \\ Ottawa, Ontaric \\ Augusc $14^{\text {th }}, 1995$
}

(C) Copyright

1995, A-Reza Neshat 
National Library

of Canada

Acquisitions and

Bibliographic Senvices Branch

395 Wellington Street

Onawa. Omano

K1A ON4
Bibliothèque nationale

du Canada

Direction des acquisitions et

des services bibliographıques

395. ne Wellinglon

Ontawa (Onterno)

rour pile vore itumence

The author has granted an irrevocable non-exclusive licence allowing the National Library of Canada to reproduce, loan, distribute or sell copies of his/her thesis by any means and in any form or format, making this thesis available to interested persons.
L'auteur a accordé une licence irrévocable et non exclusive permettant à la Bibliothèque nationale du Canada de reproduire, prêter, distribuer ou vendre des copies de sa thèse de quelque manière et sous quelque forme que ce soit pour mettre des exemplaires de cette thèse à la disposition des personnes intéressées.

L'auteur conserve la propriété du droit d'auteur qui protège sa thèse. Ni la thèse ni des extraits substantiels de celle-ci ne doivent être imprimés ou autrement reproduits sans son autorisation.

ISBN $\quad 0-612-08962-2$ 
Nom ABDOL-REZA NESHAT

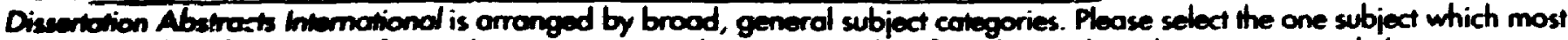
marty deveribes the combent of your dissertution. Enter the comesponding four-digit code in the spaces provided.

\section{Salind Congories}

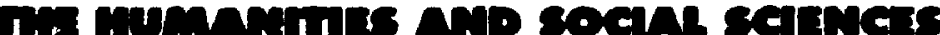

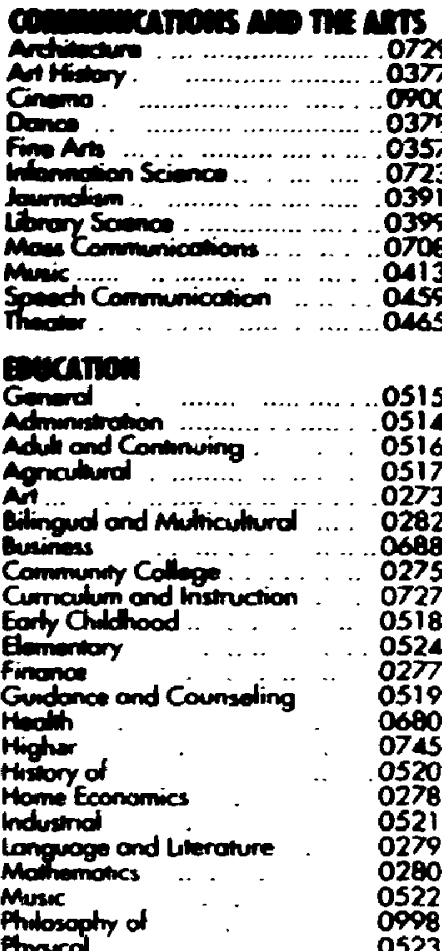

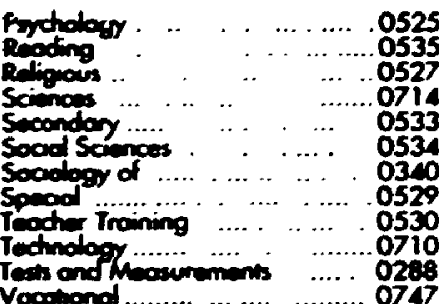

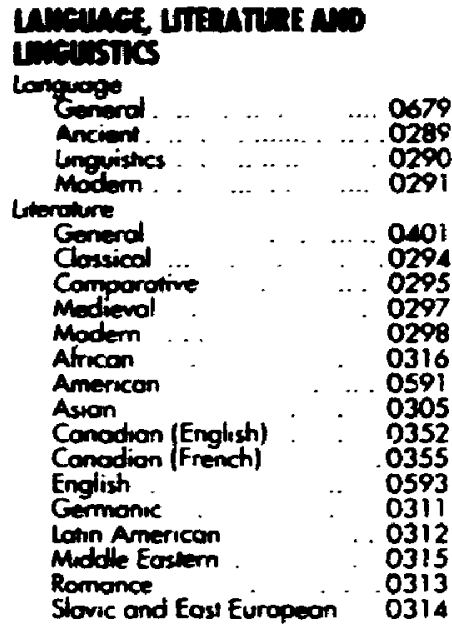

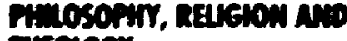
Timology

Philosophy

Religion

Etricat Sudves

Clergy

Filosophy of

Thoology

soanisainas

American Sudves

Antimupologr

Anchoeology

Cuturod

Primess Administrotion

Gerend.

Accounting

Manogement

conodion Shidies

Economion

Generol

Agriculturol

Commerce-Busines:

finonce

History

Theor

Foltulore

Googropty

Gerontology

story

Generd!
0422

0318

032

0318

032

as69

0323

0324

0326

0327

0310

0272

0770

045

0338

0385

0501

0503

0505

0508

0509

0510

0511

0358

0.366

0351

0578

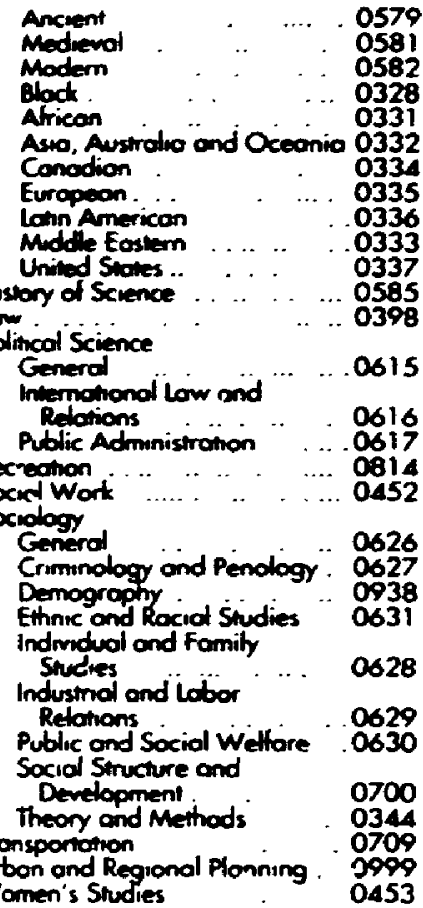

ricology

\section{Prrstcal sazmas}

Pure Sciences

Chemisiny

Gencrai

Anolyticol

Biochemisiny

Inorgonic

Nucleor

Onganic

Phomoceutical

Pinjucol

Raduotion

Mathemoincs

Physics

Generd

Acoushes

Astronomy and

Astrophysuc:

Almosphetic Serence

Alomic

Electronics and Electricity

Ekementory Porticles ond

Hugh Energy

Flud and Plosmo

Molerulor

Nucleor

Opres

Rodianon

Solid Sion

0571

0419

0382

Phormocolosy

Prriced therop

Public Hoolith

Rodiologr
Recruotion

0573

O575
Stahencs

Apiud Soninses

Applind Mechonies

Compuler science
0460
0383

0386

Engineering
Genero!

Acrospace

Agricuifuro

Automotive

Bromedicol

Chemical

Cmi

0485

0749

0486

0488

0738

0490

0491

0494

0495

075

0405

0605

0986

0606

0608

0748

$0<07$

0798

0759

0609

0810

0752

0756

0463

Electronues and Electricol

Heat and Thermodynamies

Hydrouluc

industinal

Marine

Mechonicol

Metallurgy

Mining

Nucloor

Pockoging

Sonitory and Munieipol

Sratem Scrence

Geotechnology

Operctions Reseorch

Plasncs Texhnology

Textile Technology

psravoloor

General

Astraviorol

Clinical

Derrelopmenial

Experimento

Indusitiol

Persondiny

Physologicol

Prychobiology

Piychometrics

0346

Sociol
Moterials Science
0537

0538

0540

0541

0542

O54a

0348

0545

0546

0547

0794

0548

0743

0551

0552

0549

0554

0700

0428

0796
0795

0994

0621

0384

0622

0620

0624

0625

0989

0349

0632 
The undersigned recommend to

The Faculty of Ciraduate studiess and llemearet) acceptance of the thesis

A High Order Simulation Model for the Bell 205 Helicopter submitted by

Abdol-Reza Xisliat

in partial fulfilment of the requirements for thro degree of Master of Enginmering

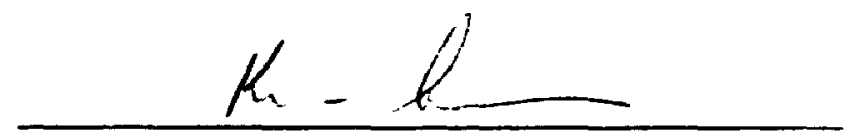

Dr. K.R. Ciohern!

Thesis Stipervisor

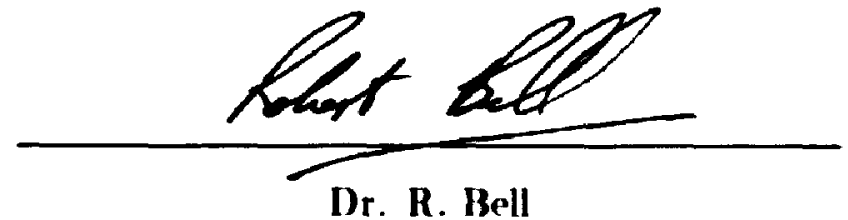

Chair. Department of Mechanical and Arosplacr linginerering

Carleton Eniversity

A ugust 30. 1995 


\section{Abstract}

A nonlinear 12 degree of freedom computer simulation is developed to simulate the Bell 205A-1 helicopter. The fuselage rotation, fuselage translation, main rotor flapping, main rotor inflow, tail rotor inflow, and the rotor-fuselage linkage dynamics are represented. A linearizing routine allows the linearization and order reduction about desired trim points.

The model is validated by comparison against actual Bell 205 flight test data and also to linear versions of the AGAJ7407 version of the manufacturer's C81 Rotorcraft Flight Simulation Computer program.

This higher order model is suitable for the design of high bandwidth, tight, feedback control systems. 


\section{Acknowledgements}

I would like to thank foremost my supervisor. Dr. K.R. Goheen, for his endless patience and guidance.

I would like to express my appreciation to Stewart Baille and Murray Morgan of the Flight Research Laboratory of the Institute for Aerospace Research. National Research Council of Canada ( NRC) for providing the flight test data, assistance on control design. and their many helpful comments. I am also grateful to Ken Hui of NRC. Dr. P. Barrington of Carleton C̈niversity. Dr. R. Kind of Carleton University, and Dr. J.H. deLeeuw of Toronto University for many conversations regarding aircraft modeling.

Financial assistance was provided by Carleton University and the NSERC scholarship program.

Special thanks must be directed to my parents, Laeya and Ali. for their unbounded understanding and generosity. 


\section{Dedication}

to Laeva and the memory of Golam-Reza 


\section{Contents}

$\begin{array}{ll}\text { Abstract } & \text { iii }\end{array}$

Acknowledgements iv

Nomenclat ure $\quad$ xiv

1 Introduction 1

1.1 Background ....................... 1

1.2 Physical Description of the NRC Bell 205 Helicopter . . . . . . . . . 2

1.3 Analysis of Coupled Rotor/Fuselage systems . . . . . . . . . . . 3

1.3.1 Mode Displacement . . . . . . . . . . . . . . . . .

1.3.2 Matrix Displacement . . . . . . . . . . . . . . . . 3

1.3.3 Force Integration . . . . . . . . . . . . . . . . .

1.4 Previous Modeling Efforts at the NRC $\ldots \ldots \ldots \ldots \ldots \ldots$

1.5 Scope of Present Work . . . . . . . . . . . . . . . . . . . ;

2 Modeling $\quad 7$

2.1 Overview of Simulator Goals $\ldots \ldots \ldots \ldots \ldots \ldots$

2.1.1 Main Rotor Flapping Dynamics . . . . . . . . . . . . . i

2.1 .2 Dynamic Inflow . . . . . . . . . . . . . . . y

2.1.3 Empennage Modeling and Main Rotor Interference . . . . . . . . 9

2.2 Reference Frames . . . . . . . . . . . . . . . . . . 10

2.3 Main Rotor . . . . . . . . . . . . . . . . . . 13 


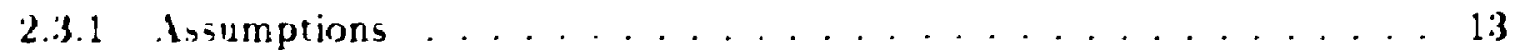

2.3.2 Tip-Path-Plane Approximation ............... 14

2.3.3 Flapping Equations of Motion . . . . . . . . . . . . . . 1.5

2.3.4 Main Rotor Aerodynamic Loads . . . . . . . . . . . . . . . $\mathbf{2 3}$

2.3 .5 Dynamic Inflow . . . . . . . . . . . . . . . 27

2.4 Tail Rotor . . . . . . . . . . . . . . . . . . . . 33

2.4 .1 Flapping Angle . . . . . . . . . . . . . . 33

2.4.2 Tail Rotor Aerodynamic Loads . . . . . . . . . . . . . 36

2.4.3 Main Rotor Interference . . . . . . . . . . . . . . . . 36

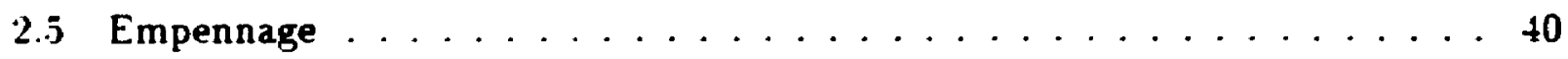

2.5.1 Horizontal Stabilizer . . . . . . . . . . . . . . . 42

2.5 .2 Vertical Fin ..................... 46

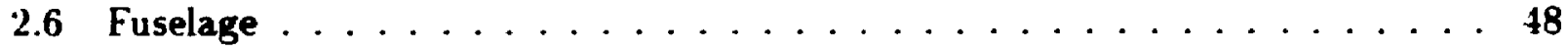

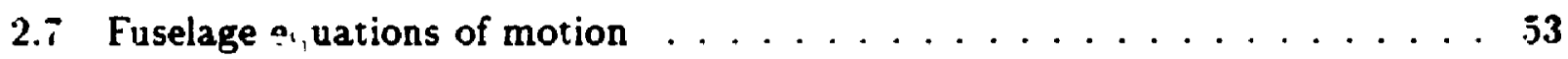

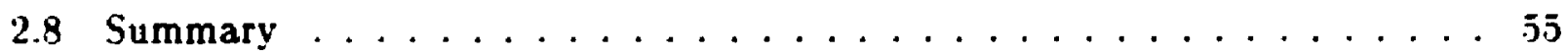

3 Computer Simulation $\quad 56$

3.1 Integration Method . . . . . . . . . . . . . . 56

3.2 Stabilization ............................

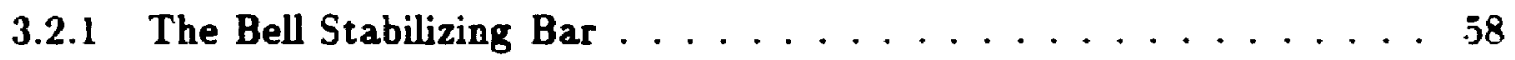

3.3 Trim Point Solution . . . . . . . . . . . . . . . . . . . 59

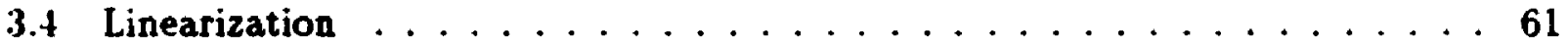

3.4.1 Model Reduction . . . . . . . . . . . . . . . 63

3.5 Summary ......................... 63

4 Model Validation $\quad 64$

4.1 Frequency Domain Method .................... 64

4.1 .1 Data Analysis . . . . . . . . . . . . . . . 65

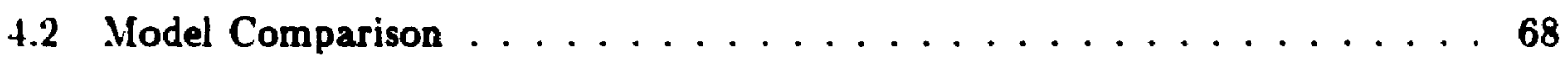

4.2 .1 On-Axis Response ......................... 69

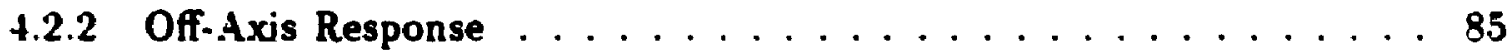


4.3 Main Rotor Fore Transmission Mlodeling . . . . . . . . . . . . . .

t.t Trim Point Verification . . . . . . . . . . . . . . . . . . 4.4

4.5 Summary . . . . . . . . . . . . . . . . a

5 Conclusions and Recommendations $\quad 100$

5.1 Conclusions . . . . . . . . . . . . . . . . . 100

5.2 Recommendations . . . . . . . . . . . . . . 101

$\begin{array}{lr}\text { APPENDICES } & 102\end{array}$

$\begin{array}{ll}\text { A Trigonometric Identities } & 102\end{array}$

B NRC Bell 205 Flight Test Data 104

B.1 Hover Data . . . . . . . . . . . . . . . . . . . . 105

B.2 40 knots Data . . . . . . . . . . . . . . . 107

B.3 80 knots Data. . . . . . . . . . . . . . . . 109

$\begin{array}{ll}\text { C Software Operating Manual } & 111\end{array}$

C.1 Compiling . . . . . . . . . . . . . . . . . . 112

C.2 Program Main Menu . . . . . . . . . . . . . . . 113

C.2.1 (1) FIND A,B MATRICES (about some trim point) . . . . . . . 113

C.2.2 (2) RUN SIMULATION USING CONTROL . . . . . . . . 114

C.2.3 (3) FIND TRIM POINT . . . . . . . . . . . . . . 11.5

C.2.4 (4) RUN SIMULATION USING NO CONTROL . . . . . . . . 115

C.3 Integration of States . . . . . . . . . . . . . 115

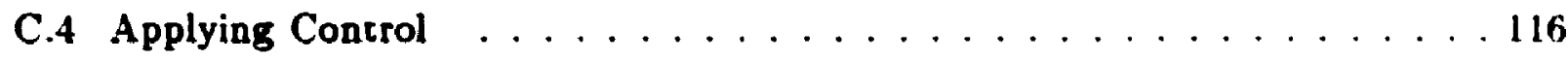

$\begin{array}{ll}\text { D Helicopter Parameters[3],(16-48] } & 120\end{array}$

E Linear Model Parameters (Part I) 124

F Linear Model Parameters (Part II) 144

$\begin{array}{ll}\text { References } & 149\end{array}$ 


\section{List of Tables}

4.1 Comparison of RBMI and Cs1 trim states . . . . . . . . . 97

4.2 Comparison of RBM and NRC Bell 205 trim states . . . . . . . . . 97

4.3 Control trim comparison with zero shift $\ldots \ldots \ldots \ldots$

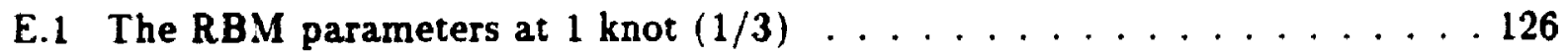

E.2 The RBM parameters at 1 knot $(2 / 3) \ldots \ldots \ldots \ldots \ldots \ldots \ldots \ldots$

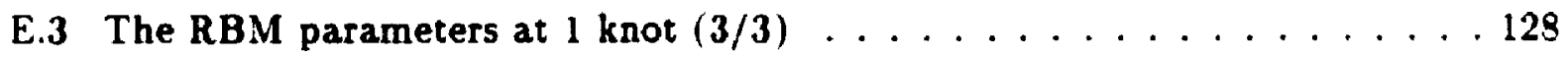

E.4 The RBM parameters at 1 knot with $\Delta v_{v_{R}}=0.01 \mathrm{~m} / \mathrm{s}(1 / 3) \ldots \ldots 12 S$

E.5 The RBM parameters at 1 knot with $\Delta v_{t_{R A}}=0.01 \mathrm{~m} / \mathrm{s}(2 / 3) \ldots \ldots 130$

E.6 The RBM parameters at 1 knot with $\Delta v_{\text {th }}=0.01 \mathrm{~m} / \mathrm{s}(3 / 3) \ldots \ldots 131$

E. 7 The RBM parameters at 40 knots $(1 / 3) \ldots \ldots \ldots \ldots$

E.3 The RBM parameters at 40 knots $(2 / 3) \ldots \ldots \ldots \ldots \ldots \ldots$

E.9 The RBM parameters at 40 knots $(3 / 3) \ldots \ldots \ldots \ldots$

E.10 The RBM parameters at 40 knots with $\Delta \bar{\phi}=0.01 \mathrm{deg}(1 / 3) \ldots \ldots 135$

E.11 The RBM parameters at 40 knots with $\Delta \bar{\phi}=0.01 \mathrm{deg}(2 / 3) \ldots \ldots \ldots$

E.12 The RBM parameters at 40 knots with $\Delta \bar{\phi}=0.01 \mathrm{deg}(3 / 3) \ldots \ldots 137$

E.13 The RBM parameters at 80 knots $(1 / 3) \ldots \ldots \ldots \ldots \ldots$

E.14 The RBM parameters at 80 knots $(2 / 3) \ldots \ldots \ldots \ldots \ldots$

E.15 The RBM parameters at 80 knots $(3 / 3) \ldots \ldots \ldots \ldots$

E.16 The RBM parameters at 80 knots with $\Delta \bar{\phi}=0.01 \operatorname{deg}(1 / 3) \ldots \ldots \ldots 14$

E.17 The RBM parameters at 80 knots with $\Delta \bar{\phi}=0.01 \operatorname{deg}(2 / 3) \ldots \ldots 142$

E.18 The RBM parameters at 80 knots with $\Delta \bar{\phi}=0.01 \mathrm{deg}(3 / 3) \ldots \ldots \ldots 1+3$ 
F.1 The RBM parameters at 1 knot with : ransmusion mode $11: 31 \ldots$. I fi

F.2 The RBII parameters at 1 knot with transmission mode $(23) \ldots \ldots$

F.3 The RB.I parameters at 1 knot with transmussion mode $(3 ; 3)$. . . . . If 


\section{List of Figures}

2.1 Body fixed axes: Fuselage(F). Shaft(S). and Shaft-Tail-Rotor(STR). . . . 11

2.2 Inertial frame to fuselage frame transformation $\ldots \ldots \ldots \ldots 11$

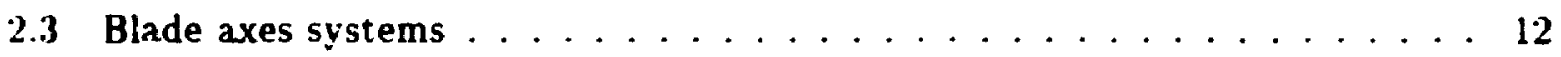

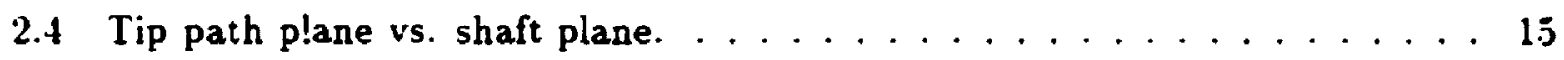

$2.5 \mathrm{Hub}$ fixed axes $\mathrm{H}$ and $\mathrm{H}^{\prime} \ldots \ldots \ldots \ldots \ldots \ldots \ldots$

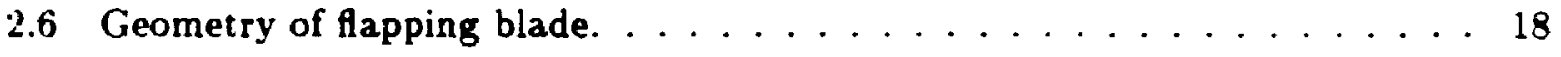

2.7 Airflow geometry at blade element $\ldots \ldots \ldots \ldots \ldots \ldots$

2.8 Ifain rotor aerodynamic loads. . . . . . . . . . . . 25

2.9 Airflow geometry at rotor disk $\ldots \ldots \ldots \ldots \ldots \ldots \ldots$

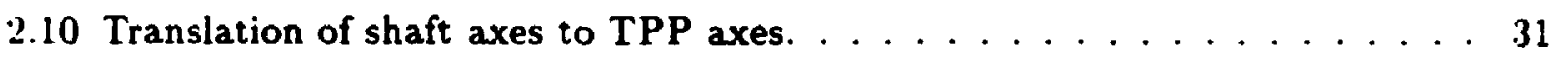

2.11 Longitudinal induced velocity ratio $(x=0)^{[33]} \ldots \ldots \ldots \ldots$

2.12 Least-squares fit of downwash at tail rotor . . . . . . . . . . 39

2.13 Typical variation of lift and drag on empennage surfaces . . . . . . . 41

2.14 Downwash variation for zero sideslip $(\mu=0.22) \ldots \ldots \ldots \ldots+\ldots \ldots$

2.15 Force/Moment geometry at horizontal stabilizer . . . . . . . . . . 4.5

2.16 Force/Moment geometry at vertical fin . . . . . . . . . . . . ti

2.17 Force/Moment geometry at fuselage $\ldots \ldots \ldots \ldots \ldots$

2.18 Fuselage models $\ldots \ldots \ldots \ldots \ldots \ldots \ldots \ldots \ldots \ldots$

2.19 Phasing between low and high angle models $\ldots \ldots \ldots \ldots \ldots \ldots$

3.1 Schematic of the Bell Stabilizer Bar ${ }^{[25]} \ldots \ldots \ldots \ldots \ldots$

3.2 Schematic of the Bell stabilizer bar control law . . . . . . . . . . 60 
3.3 Response to a one second step input at hoter .............. (ill

3.t Response to a step input at 20 knots . . . . . . . . . . . . . (it

4.1 Typical frequency sueep pattern ................. tii

4.2 YRC Bell 205 longitudinal cyclic to pitch rate response at hoter . . . . . iv

4.3 Longitudinal cyclic to pitch rate at hover . . . . . . . . . . . . . . ill

4.4 Longitudinal cyclic to pitch rate at hover $\left(\Delta r_{\zeta_{R}}=0.01 \mathrm{~m} / \mathrm{s}\right) \ldots \ldots$

4.5 Longitudinal cyclic to pitch rate at 40 knots . . . . . . . . . . . . .

4.6 Longitudinal cyclic to pitch rate at 80 knots . . . . . . . . . . . il

4.7 Lateral cyclic to roll rate at hover . . . . . . . . . . . . . . .

4.8 Lateral cyclic to roll rate at 40 knots . . . . . . . . . . . . . . . is

4.9 Lateral cyclic to roll rate at 80 knots . . . . . . . . . . . . . . . . 3

4.10 Pedal to yaw rate at hover . . . . . . . . . . . . . . . it

4.11 Pedal to yaw rate at 40 knots ... . . . . . . . . . . . . . . . .

4.12 Pedal to yaw rate at 80 knots ... . . . . . . . . . . . . . .

4.13 Pedal to yaw rate at 40 knots $(\Delta \bar{\phi}=0.01 \mathrm{deg}) \ldots \ldots \ldots$

$4.1+$ Pedal to yaw rate at 80 knots $(\Delta \bar{\phi}=0.01 \mathrm{deg}) \ldots \ldots \ldots$

4.15 Collective to vertical acceleration at hover . . . . . . . . . . . . . it

4.16 Collective to vertical acceleration at 40 knots . . . . . . . . . is

4.17 Collective to vertical acceleration at 80 knots . . . . . . . . . . . 8

4.18 Collective to vertical acceleration at 40 knots $(\Delta \bar{\phi}=0.01 \mathrm{deg}) \ldots \ldots$

4.19 Collective to vertical acceleration at 80 knots $(\Delta \bar{\phi}=0.01 \mathrm{deg}) \ldots \ldots$

4.20 Longitudinal cyclic to forward acceleration at hover . . . . . . . . . 0

4.21 Longitudinal cyclic to forward acceleration at 40 knots . . . . . . . . . \$ü

4.22 Longitudinal cyclic to forward acceleration at 80 knots . . . . . . . . . 81

4.23 Longitudinal cyclic to forward acceleration at 40 knots $(\Delta \vec{\phi}=0.01 \mathrm{deg}) \quad$. 82

4.24 Longitudinal cyclic to forward acceleration at $80 \mathrm{knots}(\Delta \bar{\phi}=0.01 \mathrm{deg}) \quad . \quad 82$

4.25 Lateral cyclic to lateral acceleration at hover . . . . . . . . . . . . . \3

4.26 Lateral cyclic to lateral acceleration at 40 knots . . . . . . . . . . . \$.3

4.27 Lateral cyclic to lateral acceleration at 80 knots . . . . . . . . . . . . 44 
4.29 Lateral cyclic to lateral acceleration at 40 knots $(\Delta \overline{0}=0.01 \mathrm{deg}) \ldots \ldots$

4.29 Lateral cyclic to lateral acceleration at $80 \operatorname{knots}(\Delta \bar{\phi}=0.01 \mathrm{deg}) \ldots . . .85$

1.30 Pedal to lateral acceleration at hover . . . . . . . . . . . . . 86

4.31 Pedal to lateral acceleration at 40 knots . . . . . . . . . . . . 86

1.32 Pedal to lateral acceleration at 80 knots $\ldots \ldots \ldots$

$\$ .33$ Longitudinal cyclic to roll rate at hover . . . . . . . . . . . . . 87

4.34 Longitudinal cyclic to roll rate at 40 knots . . . . . . . . . . . . . 88

4.35 Longitudinal cyclic to roll rate at 80 knots . . . . . . . . . . . . . . 88

4.36 Lateral cyclic to pitch rate at hover . . . . . . . . . . . . . . 89

4.37 Lateral cyclic to pitch rate at 40 knots . . . . . . . . . . . . . . . . . 90

4.38 Lateral cyclic to pitch rate at 80 knots . . . . . . . . . . . . . 90

4.39 Collective to yaw rate at hover ... . . . . . . . . . . . 91

4.40 Collective to yaw rate at 40 knots . . . . . . . . . . . . . . . 91

4.41 Collective to yaw rate at 80 knots . . . . . . . . . . . . . . . . 92

4.42 Longitudinal transmission mode . . . . . . . . . . . . . . . . 93

4.43 Longitudinal cyclic to pitch rate (hover) . . . . . . . . . . . . . 95

4.44 Longitudinal cyclic to forward acceleration (hover) . . . . . . . . . . 95

4.45 Lateral cyclic to pitch rate (hover) . . . . . . . . . . . . . . . 96

B.1 Lateral channel frequency sweep . . . . . . . . . . . . . 105

B.2 Longitudinal channel frequency sweep . . . . . . . . . . . 105

B.3 Vertical and Yaw channel frequency sweeps . . . . . . . . . . 106

B.4 Lateral channel frequency sweep . . . . . . . . . . . . . . 107

B.5 Longitudinal channel frequency sweep . . . . . . . . . . . . . 107

B.6 Vertical and Yaw channel frequency sweeps . . . . . . . . . . . 108

B.7 Lateral channel frequency sweep . . . . . . . . . . . . . . . . . . 109

B.8 Lougitudinal channel frequency sweep . . . . . . . . . . . . . I09

B.9 Vertical and Yaw channel frequency sweeps . . . . . . . . . . . 110

C.1 Sudden forward velocity command of $8 \mathrm{~m} / \mathrm{s}$ from hover . . . . . . . 119 


\section{Nomenclature}

\begin{tabular}{|c|c|}
\hline$a$ & lift curve slope \\
\hline .4 & disk area \\
\hline $.1=$ & lateral cyclic pitch \\
\hline$a_{0}$ & lift curve slope at zero sideslip \\
\hline A, & longitudinal cyclic pitch \\
\hline$b$ & number of blades \\
\hline BL & buttline \\
\hline$B P$ & tip-loss factor \\
\hline$c$ & blade cord \\
\hline$C_{1}$ & rotor aerodynamic rolling moment coefficient \\
\hline$C_{2}$ & rotor aerodynamic pitching moment coefficient \\
\hline$c_{1}$ & profile drag coefficient \\
\hline$\left(c_{d_{0}}, c_{d_{1}}, c_{d_{2}}\right)$ & profile drag constants \\
\hline$C_{D}$ & drag coefficient \\
\hline$C_{D_{p}}$ & profile drag coefficient \\
\hline$C_{L}$ & surface lift coefficient \\
\hline$C_{L_{m}}$ & maximum lift coefficient of airfoil \\
\hline$c_{0}$ & built in precone angle of the blade-hub assembly \\
\hline$c$ & bar to hiade linkage factor of Bell stabilizer bar \\
\hline$C_{T}$ & thrust coefficient \\
\hline $\mathbf{D}$ & airfoil profile drag \\
\hline$D_{\alpha=90^{\circ}}$ & fuselage drag at $\alpha=90^{\circ}$ \\
\hline$D_{3=90}$ & fuselage drag at $\beta=90^{\circ}$ \\
\hline$D, Y, L$ & wind axes drag, sideforce, and lift \\
\hline$d D_{s}$ & component of differential profile drag in rotor plane \\
\hline$d D$ & blade element differential profile drag \\
\hline$d \mathcal{F}$ & total differential force acting on blade element $=d \vec{C}+d \vec{D}$ \\
\hline
\end{tabular}




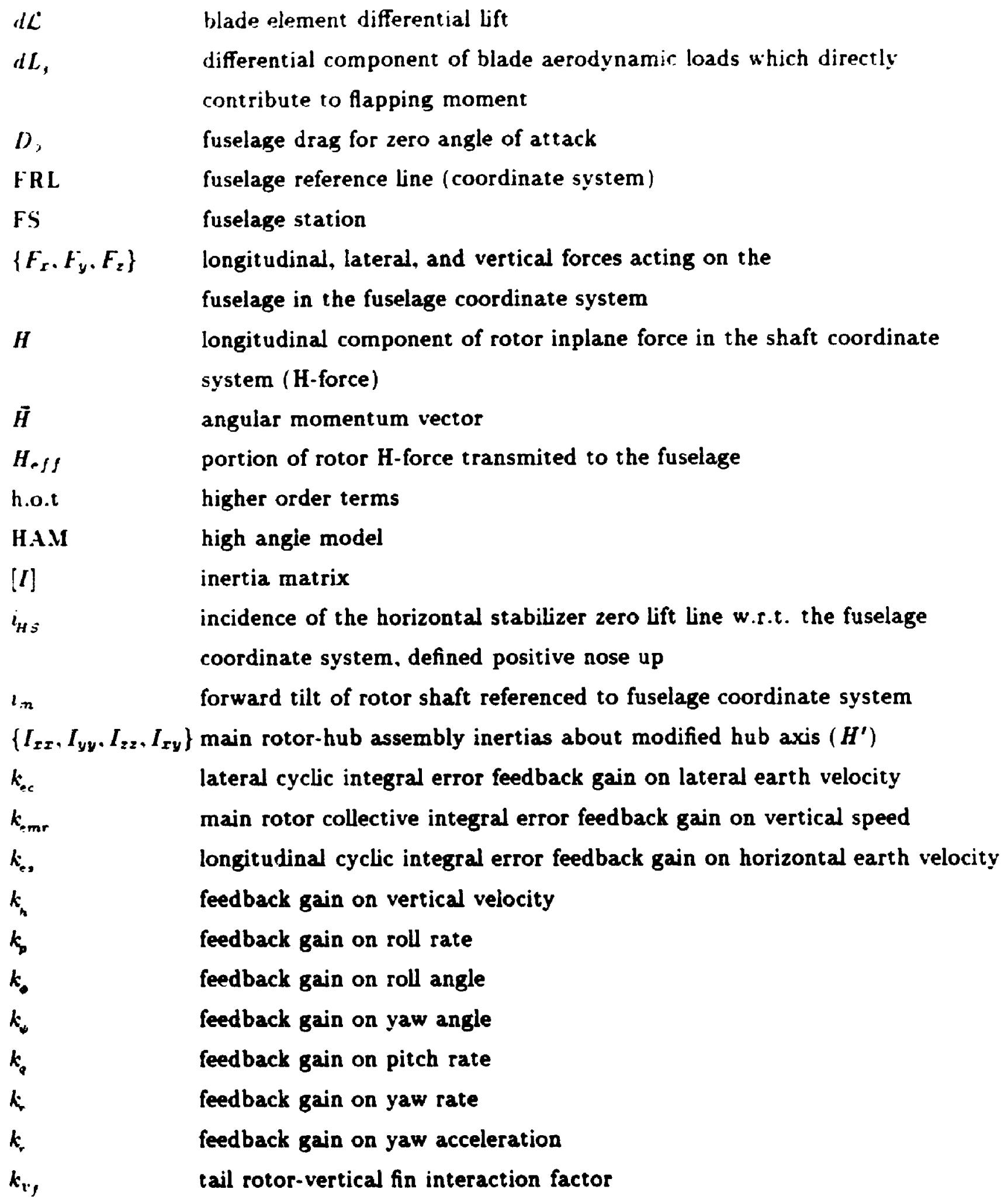




\begin{tabular}{|c|c|}
\hline$k_{9}$ & feedback gain on pitch angle \\
\hline$k_{=:}$ & feedback gain on horizontal earth velocity \\
\hline$k_{y e}$ & feedback gain on lateral earth velocity. \\
\hline$l_{j=90^{\circ}}$ & fuselage rolling moment at a sideslip angle of $3=90^{\circ}$ \\
\hline $\mathbf{L}$ & airfoil lift \\
\hline$[\dot{L}]$ & $\begin{array}{l}\text { nonlinear version of the inflow gain matrix translated to the } \\
\text { TPP-body coordinate system }\end{array}$ \\
\hline$\{l, . M, . V\}$ & wind axes roiling , pitching, and yawing moments respectively \\
\hline L.A.M & low angle model \\
\hline$L_{0}$ & fuselage lift for zero angle of attack $(\alpha)$ \\
\hline$m_{2}$ & $\begin{array}{l}63.7 \% \text { of the air mass of the circumscribed sphere defined by the tail } \\
\text { rotor radius }\end{array}$ \\
\hline $.1 K_{\alpha=90^{\circ}}$ & fuselage pitching moment at an angle of attack of $\alpha=90^{\circ}$ \\
\hline.$H_{3}$ & rotor flapping moment \\
\hline$[. M]$ & apparent mass matrix \\
\hline$M_{0}$ & fuselage pitch moment for zero angle of attack $(\alpha)$ \\
\hline$\left\{M_{x}, M_{y}, M_{z}\right\}$ & rolling, pitching, and yawing moments respectively \\
\hline.$v_{3=90^{\circ}}$ & fuselage yawing moment at a sideslip angle of $3=90^{\circ}$ \\
\hline$\{p, q, r\}$ & $\begin{array}{l}\text { roll, pitch, and yaw angular velocities of the fuselage in the fuselage } \\
\text { coordinate system }\end{array}$ \\
\hline$P \cdot M$ & aerodynamic pitching moment of the rotor \\
\hline$Q$ & dynamic pressure \\
\hline$q$ & fuselage dynamic pressure \\
\hline QSM & the quasi-static model of reference \\
\hline$\vec{r}$ & position vector \\
\hline $\boldsymbol{R}$ & main rotor radius \\
\hline RBM & the rotor body model developed in this thesis \\
\hline R.M & aerodynamic rolling moment of the rotor \\
\hline$S$ & tail rotor disk area blocked by the vertical fin \\
\hline$S_{V F}$ & planform area of vertical fin \\
\hline
\end{tabular}




\begin{tabular}{|c|c|}
\hline$t$ & time \\
\hline$T$ & thrust \\
\hline$T_{4}$ & transformation matrix (shaft to TPP-body) \\
\hline$\Gamma_{B \cdot R}$ & transformation matrix (rotor plane to supplementary blade) \\
\hline$I_{f}$ & Bell stabilizer bar time constant \\
\hline$T_{F I}$ & transformation matrix (earth to fuselage) \\
\hline$T_{F W}$ & transformation matrix (wind to fuselage) \\
\hline$T_{H A}$ & transformation matrix (rotor plane to hub) \\
\hline$T_{\text {net }}$ & tail rotor net thrust \\
\hline$T_{R S}$ & transformation matrix (shaft to rotor plane) \\
\hline$T_{S F}$ & tra. :formation matrix (fuselage to shaft) \\
\hline$T_{S_{T R} F}$ & transformation matrix (fuselage to tail rotor shaft) \\
\hline $\bar{u}$ & control vector \\
\hline $\bar{u}$ & perturbation control vector \\
\hline$\left\{u_{F}, v_{F}, w_{F}\right\}$ & fuselage velocity components in fuselage coordinate system \\
\hline$\left\{u_{H S}, v_{H S}, w_{H S}\right\}$ & horizontal stabilizer center of pressure velocity components \\
\hline $\bar{u}_{o}$ & trim control vector \\
\hline$\left\{U_{R}, U_{T}, U_{P}\right\}$ & $\begin{array}{l}\text { relative wind velocities of blade element in radial, tangential, and } \\
\text { normal directions respectively }\end{array}$ \\
\hline $\bar{u}_{\text {TRSM }}$ & trim control vector \\
\hline$\left\{u_{i F}, v_{v F}, w_{v F}\right\}$ & velocity components of the vertical fin in fuselage system \\
\hline $\boldsymbol{V}$ & total velocity at blade element \\
\hline$\bullet$ & inflow mass-flow parameier \\
\hline$V^{\prime \prime}$ & total velocity through tail rotor \\
\hline$[l]$ & mass flow parameter matrix \\
\hline $\bar{V}_{F}$ & total velocity at the fuselage aerodynamic reference point \\
\hline$v_{H S}$ & lateral velocity component at horizontal stabilizer center of pressure \\
\hline$l_{\text {its }}$ & center of pressure velocity of the horizontal stabilizer $=\left\{u_{H S}, v_{H S}, w_{H S}\right\}$ \\
\hline$v_{1}$ & momentum theory main rotor induced velocity $=\lambda_{m} \cdot(\Omega R)$ \\
\hline r & an tail rotor induced velocity \\
\hline
\end{tabular}


$\vec{r}_{R}$

$v_{T}$

$r_{T R}$

$\vec{r}_{V F}$

$u$

$w_{\text {HS }}$

$u_{\text {TR }}$

$u_{w F}$

WL

$x$

$\overline{\boldsymbol{x}}$

$\hat{\boldsymbol{x}}$

$\left\{X_{F H S}, Y_{F H S}\right.$,

$\left.Z_{F H S}\right\}$

$\left\{X_{F V F}, Z_{F V F}\right\}$

$x_{0}$

$\bar{x}_{0}$

$X_{S F}$

$X_{S H S}$

$X_{S_{T R} F}$

$\bar{x}_{T R I N}$

$Y$

Yesf

$Z_{s F}$

$Z_{S H}$

$Z_{S_{T R^{F}}}$ resultant normalized velocity of oncoming flow at rotor

total resultant flow through the rotor disk

velocity of oncoming flow at tail rotor

center of pressure velocity of the vertical fin

coordinate frame angular velocity

main rotor downwash at the left and right horizontal stabilizers

main rotor downwash at tail rotor

main rotor downwash at the vertical fin. defined positive down

water line

non-dimensional span distance $r / R$

state vector

perturbation state vector

$x, y$, and $z$ displacement of the horizontal stabilizer center of pressure from fuselage c.g.

$x$ and $z$ displacement of the vertical fin center of pressure from fuselage c.g.

root cutout factor (non-dimensional radius to inner blade location)

trim state vector

longitudinal distance between fuselage c.g. and flapping hinge of main rotor

$x$ location of the horizontal stabilizer measured in the Hub-body coordinate system

longitudinal distance between fuselage c.g. and tail rotor flapping hinge trim state vector

component of rotor inplane force in shaft coordinate system (Y-force)

portion of rotor $\mathrm{Y}$-force transmited to the fuselage

vertical distance between fuselage c.g. and main rotor flapping hinge

hub undersling

vertical distance between fuselage c.g. and tail rotor flapping hinge 


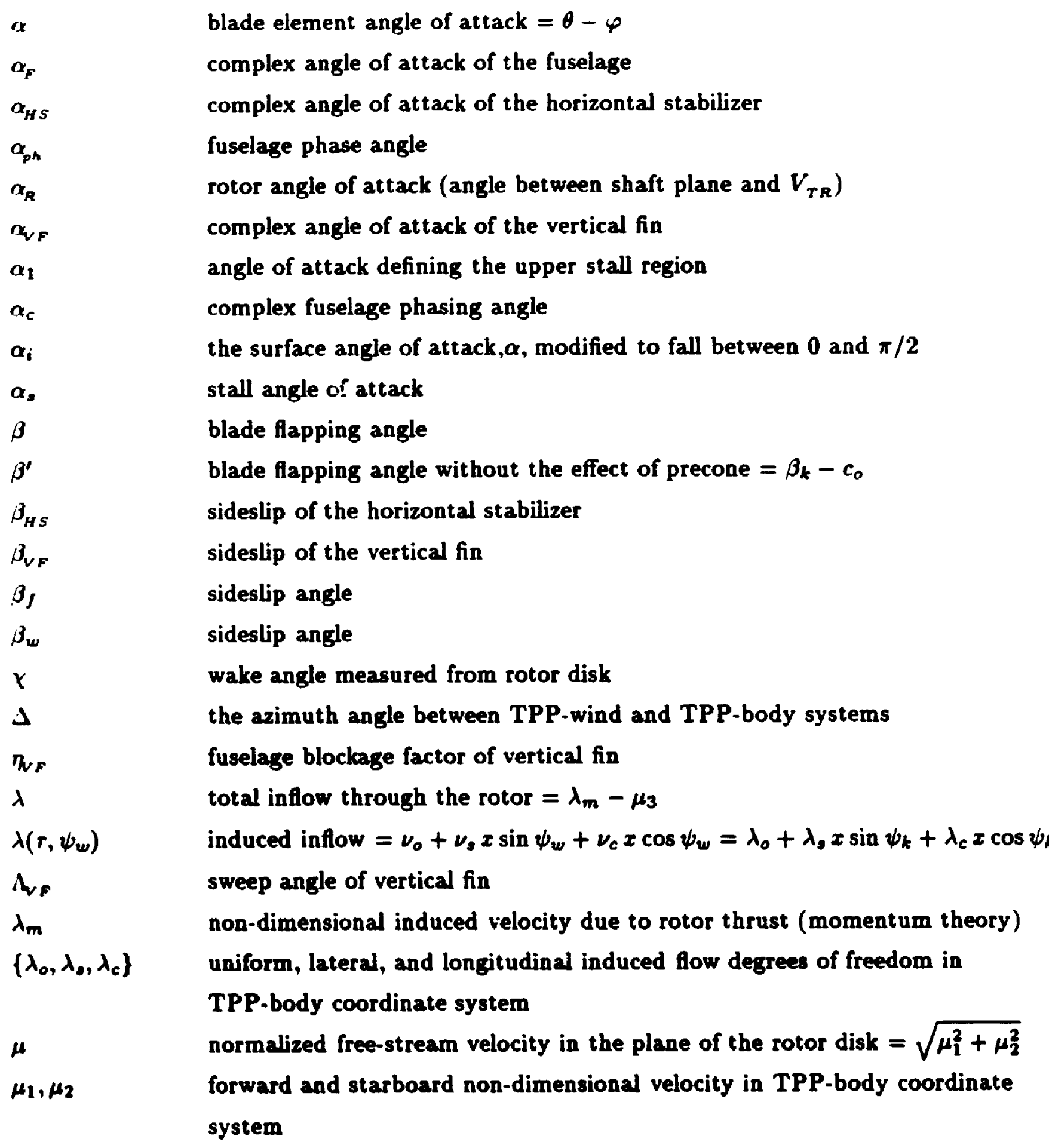


$\mu_{3}$

$\left\{\mu_{x}+\mu_{y}, \mu_{z}\right\}$

$\left\{\nu_{2}, \nu_{3} \cdot \nu_{s}\right\}$

$\Omega$

$\Phi$

$O_{R}$

$\Psi$

$u_{k}$

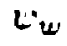

$\rho$

$\sigma$

$\theta$

$\Theta$

$\theta_{\text {R }}$

$\theta$ 。

$\theta_{t}$

$\uparrow$

\section{Subscripts}

$B^{\prime}$

F

H

$H^{\prime}$

HS

I

k

MR

R vertical non-dimensional velocity in TPP-body coordinate "yatem normalized wind velocity at the hub and in the shaft coordinate system uniform. lateral. and longitudinal wake degrees of freedom in IPP-wind coordinate system

rotor angular velocity $=\frac{t_{u k}}{1 t}$

Euler roll angle

lateral flapping angle

Euler yaw angle

$\mathrm{k}^{\text {th }}$ blade azimuth angle

rotor azimuth measured in TPP-wind coordinate system

air density

blade solidity

blade pitch angle measured from blade hub $=\theta_{o}+\theta_{t} x+A_{c} \cos \psi_{k}{ }^{\prime}+A_{g} \sin \psi$

Euler pitch angle

longitudinal flapping angle

blade root collective pitch measured from hub plane

linear twist of blade (blade tip twist w.r.t. blade root)

blade inflow angle

supplementary blade

fuselage

hub axes

hub axes shifted to flapping hinge

horizontal stabilizer

inertial

$\mathbf{k}^{\text {th }}$ blade

main rotor

rotor/rotor plane 


$\begin{array}{ll}S & \text { ihaft } \\ S T R & \text { tail-rotor shaft } \\ I R & \text { tail rotor } \\ V F & \text { vertical fin } \\ u & \text { wind axes }\end{array}$

Special . Votation

(i) differentiation with respect to rotor azimuth

${ }_{\theta^{\prime}} \vec{x}_{P} \quad$ the absolute velocity vector of point $\mathrm{P}$ resolved in the $\mathrm{B}^{\prime}$ coordinate system

$\mathbb{B}^{\prime} \bar{v}_{P / S}$ the velocity vector of point $P$ relative to point $A$ resolved in the $B^{\prime}$ coordinate system

$\vec{w}_{F / I}$

$\bar{r}_{s / F}$ absolute angular velocity of the $F$ coordinate system resolved in the $F \cdot f$ ame position vector from point $F$ to point $S$ 


\section{Chapter 1}

\section{Introduction}

\subsection{Background}

Present day requirements on helicopter performance and pilot workload place greater emphasis on "tight" high-bandwidth flight control systems. Low order quasi-static helicopter models which ignore the dynamics of the rotor and the inflow through it have been used for many years to design feedback control systems. However, these models limit the effective bandwidth of the controllers, and are unsuitable for the design and evaluation of high-bandwidth systems ${ }^{(1,2)}$.

For over 25 years, a Bell 205A-1 (Bell 205) helicopter has been used for airborne simulation, automatic control, and handling qualities research at the Flight Research Laboratory of the Institute for Aerospace Research, National Research Council of Canada (NRC). The alteration of the Bell 205 dynamics to represent that of other aircraft or simply control design and evaluation requires the use of a mathematical model which represents the openloop characteristics of the "raw" helicopter. Until now, a low order 6 degree of freedom (6 D.O.F.) stability derivative model which lacks the rotor and inflow degrees of freedom has been used for this purpose. In addition, this model, as presented in reference [3], represents a Bell UH-1H helicopter which, although similar to the Bell 205, has some differences whuch affect both trim attitude and dynamic response.

In this study a computer simulation for a Bell 205A-1 helicopter is developed from which higher order linear state space models can be extracted and used for design purposes. 
The resulting designs can then be tested on the nonlinear simulation prior to evaluation on the actual helicopter.

\subsection{Physical Description of the NRC Bell 205 Helicopter}

The NRC Bell 205 helicopter is a modified version of the Bell 205A-1 general purpose helicopter. The standard Bell 205A-1 has an all-aluminum teetering main rotor on an underslung feathering axis hub with a stabilizer bar mounted at right anglr 3 to the main rotor blades to increase dynamic stability. The fuselage is all-metal semi-monocoque and is powered by a single Lycoming $\mathrm{T5313A}$ turboshaft engine. The anti-torque tail rotor is right side mounted (tractor configuration) and is also of the teetering type.

Many modifications have been made to the basic aircraft by NRC since its purchase in 1969. A detailed description of these modifications can be found in reference [4]. The changes which affect the dynamics of the aircraft are:

1. Addition of the necessary hardwart and software to allow full authority fly $y$-wire actuation. The evaluation pilot's control inputs are directed to the onboard computers which generate the necessary full authority fly-by-wire actuator commands necessary to control the aircraft.

2. Removal of the stabilizer bar to provide increased aircraft contsol authority at the expense of reduced dynamic stability.

3. Removal of the original tail-rotor assembly and installation of a Bell 212 wide-cord, right side mounted tail-rotor. This modification, which improved yaw axis control, was a result of an airworthiness directive for all commercial Bell 205 helicopters.

4. The fixed longitudinal cyclic io horizontal stabilizer gearing was replaced by an electro-hydraulic actuator allowing the stabilizer to be programmed as a second source for pitch col. rol.

A general schematic of the NRC Bell 205 is shown in figure 2.1 and the pertinent parameters are listed in Appendix D. 


\subsection{Analysis of Coupled Rotor/Fuselage systems}

The rotor ard airframe of a helicopter comprise a coupled dynamic system. Sieveral approaches are available to perform a coupled rotor-fuselage analysis. Among these, the most popular are Mode Displacement. Matrix displacement. and Force Integration methods.

\subsubsection{Mode Displacement}

In the mode displacement method, the modal method of structural analysis is used to describe the rotor. The blade natural frequencies, mode shapes, and associated coefficients are first derived using a technique such as a finite element method.

A detailed analysis of the coupled rotor-fuselage system will yield inertial coupling between the rotor and fuselage in both sets of equations. The mode displacement method, however, simplifies the equations by replacing rotor inertia couplings in the fuselage equations with simpler stiffness type couplings and thus eliminating the acceleration terms in the fuselage equations that are due to rotor degrees of freedom ${ }^{[5]}$. The need to simultaneously solve all equations of motion has been removed in favor of a simpler sequential solution of the coupled equations. The drawback of this method is that other techniques are required to calculate the modal coefficients and also this method tends to be more suitable for helicopter vibration analysis.

\subsubsection{Matrix Displacement}

The Matrix Displacement method allows the analysis of structural components in rotating and nonrotating reference frames by applying a generalized coupling procedure. This procedure is outlined in reference [5].

The equations of motion are derived for each component of a coupled system separately ignoring the constraints and then the system is coupled by transforming the states using a coupling matrix which is derived by multiplying a coordinate transformation matrix by a constraint relationship matrix. The resulting stiffness, mass, and damping matrices are time dependent and require algebraic manipulation by the computer at each time point. Potential disadvantages of this method are longer computation time and poor numerical accuracy due to a lack of complete cancellation of terms. In addition, this method is geared toward linear analysis. 


\subsubsection{Force Integration}

Is stated earlier the rotor equations of motion contain inertial coupling terms due to the fuselage. and thus rotor shaft. motions. In the Force Integration method the fuselage accelerations are assumed to be known at a particular time of interest. In order to solve the rotor equations of motion a time $t$, the necessary fuselage accelerations are obtained from the sulution of the non-rotating system (fuselage) at the previous time point. It is standard to use some type of predictor-corrector method for numerical integration of rotor accelerations. In order to solve the fuselage equations, the hub shears and moments are required. These are obtained by integrating the aerodynamic and inertial loadings from blade root to blade tip. The force integration is performed at a time for which the predictor-corrector has converged to a solution of the rotor equations. The fuselage equations can now be solved giving the hub motions required in the rotor equations of the next time point.

The disadvantages of this method are excessive computer time and an occasional numerical instability which is due to an unfavorable apparent mass-ratio between the rotating and non-rotating system. This method. however. accurately accounts for the rotor fuselage coupling and is favorable for calculating the response of a coupled rotor fuselage system. In this thesis, with the help of the symbolic processor Mathematica, the Force Integration method is used to simulate the Bell 205 helicopter.

\subsection{Previous Modeling Efforts at the NRC}

The modeling efforts at the NRC have been geared towards parameter estimation methods for identification of aerodynamic derivatives from flight test data.

The work essentially started with Gould ${ }^{[6]}$ in 1973. The lateral-directional stability derivatives of the Bell 205 were estimated by a least squares quasilinearization method which employed a priori estimates for the derivatives to help convergence. Higher order effects were not considered and the quasi-steady body stability derivatives were only approximated at three speeds.

The next major effort was credited to J.H. deLeev and K. Huil?]. In this wcrk, the linear maximum likelihood parameter estimation method, based on a linear aircraft model, was used to estimate the complete 6 D.O.F. quasi-steady rigid body stability 
derivatives for the Bell 205 and Bell 206 helicopters. This procedure effectively varies the coefficients of the adopted model. in a structured fashion. to obtain a best fit to the Hight test data. Again. higher order dynamics were neglected and the shortcomings in the effort was stated to be the exclusive use of a linear base model. lack of rotor dynamics. lack of inflow dynamics. and omission of engine governor behavior.

Later efforts by hui and Baillie ${ }^{[8]}$ involved the incorporation of simple rotor dynanics to improve the validity of the parameter estimation model for a Bell $412 \mathrm{HP}$ helicopter. As in $[i]$, the maximum likelihood parameter estimation method was first used with a 6 D.O.F quasi-steady rigid body base model to arrive at the stability derivative estimates. It was shown that models derived in this fashion predicted body responses to control inputs whirh were always ahead of measured body responses. The deficiency in the estimated response, being due in part to rotor dynamics, was alleviated by addition of rotor flapping effects. This was accomplished by using a simple theoretical rotor flapping model to generate the rotor flapping states from the available flight data prior to stability derivative estimation of an 8 D.O.F. rigid body-rotor flapping model. It was concluded that with rotor dynamics, the estimated model possessed superior prediction capabilities.

\subsection{Scope of Present Work}

The nonlinear equations for the flapping motion of a teetering rotor at arbitrary speeds and with non-flexible blades are derived in chapter 2 using quasi-steady aerodynamic theory and the tip-path-plane approximation. The rotor thrust, drag, and torque are derived with the assumption that compressibility and reverse flow effects are small. Dynamic inflow is used to describe the induced flow for both rotors and the induced flow interaction with the fuselage and empennage surfaces are accounted for. The aerodynamic loads on the fuselage, vertical fin, and horizontal stabilizer are approximated for all angles of attack and sideslip.

Chapter 3 outlines the numerical integration technique used to solve the differential equations describing the aircraft. In addition, the state feedback control laws used to stabilize and command the simulation are defined. A simple Taylor series expansion is used to numerically linearize the nonlinear equations of motion.

In chapter 4 , the model is compared to the 6 D.O.F. quasi-static model of [3] and 
also to flight test data obtained from the Flight Research Laboratory of the NRC. A frequency-domain identification approach for modeling of the flexible rotor-fuselage interface is proposed and evaluated.

Finally, chapter 5 presents the conciusions and recommendations for future work. 


\section{Chapter 2}

\section{Modeling}

This chapter outlines the development of the equations of motion for a single teetering rotor helicopter. The dynamics of the main rotor, fuselage, and rotor induced velocity are represented. In addition, the aerodynamic loads on the fuselage and empennage are approximated for all angles of attack and sideslip including vertical and rearward flight.

\subsection{Overview of Simulator Goals}

In order for the computer model to be useful in both handling qualities simulation and control applications, it must represent the major modes of the aircraft. The main features that are incorporated into the model are listed bellow followed by an explanation as to their importance.

1. Main rotor flapping dynamics;

2. Main rotor dynamic inflow;

3. Empennage modeling and main rotor interference effects.

\subsubsection{Main Rotor Flapping Dynamics}

Chen ${ }^{[9]}$ shows that the flapping dynamics should be considered in assessing the flight dynamics and handling qualities of hingeless rotor helicopters or helicopters with heavy rotor blades. The three natural modes of an aiticulated rotor are the coning, advancing, and the regressing modes. The coning mode can be ignored for a teetering rotor since the blades are rigidly coupled. Using Chen's analysis it is possible to show that for 
the NAE Bell 205 the regressing and advancing flapping modes have undamped natural frequencies of $13.5 \mathrm{rad} / \mathrm{sec}$ and $64.5 \mathrm{rad} / \mathrm{sec}$, respectively. Of these modes, the regressing flapping mode is most significant since its frequency is low enough to alter the fuselage modes thereby effecting the handling characteristics of the aircraft. The impact of the advancing mode on the aircraft handling characteristics is considerably less due to its high frequency (of the order of two times the rotational speed of the main rotor). For a complete simulation, at least the regressing fapping mode should be modelled.

The six degree of freedom model presently used at NRC as presented in Heffley[3] uses a well known quasi-static rotor assumption which removes the dynamic characteristics of the tip-path-plane by assuming the rotor plane to have the same angular velocity as the rotor shaft; cyclic control applications are instantaneously converted to tip-path-plane tilt angles. This simplification is adequate for analysis associated with low frequency (such as the phugoid or low-gain feedback design) and steady state flight dynamics. Ellis ${ }^{[10]}$ shows that any analysis of high gain attitude and angular rate feedback should include the dynamics of the rotor. Heimbold ${ }^{[1]}$ staies that the quasi-static rotor assumption is inadequate because the flapping regressive modes are not accounted for giving the deceptive impression of greater aircraft stability. In [1] and [12] the quasi-static rotor model is directly compared to models that include rotor dynamics; it has been found that a complete negligence of the rotor dynamics in the aircraft control design leads to deteriorated response and sometimes produces unstable closed-loop response on the model with rotor dynamics. Hall[1] concluded that the design of tight controllers required the inclusion of rocor dynamics. It is shown that the relative bandwidth ${ }^{1}$ of an eighth order model which includes the tip-path-plane dynamics is considerably higher than the conventional 6 D.O.F. model without rotor dynamics[13]. In addition the 6 D.O.F. model tends to incorrectly predict the initial transient response and the phase response for the subsequent motion.

\subsubsection{Dynamic Inflow}

An important part of helicopter modeling is calculating the induced-velocity of air through the rotor disc. Not only does the induced-velocity directly affect flapping dynamics and

\footnotetext{
'defined a that bandwidth for which a given model will duplicate the true frequency reaponse characteristics of the ectual aireraft
} 
rotor forces but it also impinges upon surfaces of the fuselage (vertical/horizontal stabilizers. tail rotor. and the fuselage itself ) to alter fuselage aerodynamics. References [1.1] to ii! outline the evolution of induced-velocity research starting with the pioneering work of Glauret in 1926 and ending with the model used in this thesis. the Pitt-Peters dynamic inflow model.

In reference [18] a linear analysis was carried out to determine the effect of dynamic inflow on the vertical response of a helicopter. Good agreement was observed between the model and flight test data. It was noted that the model predicts well the initial vertical acceleration overshoot to a step collective input and is far superior to a quasi-static inflow model (no dynamic inflow). Goankar ${ }^{[17]}$ compares the Pitt-Peters dynamic inflow model to flight test data and to other models which are not dynamic in nat::e. They conclude that dynamic inflow is necessary for correlation with flight test data and that the Pitt-Peters model appears to be the best currently available model for rotor analysis. In reference [19] a linear flight simulation program was developed which included the Pitt-Peters inflow model. They concluded that dynamic inflow plays an important part for correlation in hover but is not as important in forward flight.

It was stated earlier that for high gain control applications it becomes necessary to model higher-order dynamics such as rotor flapping. References [16] and [17] suggest that the frequencies of the inflow dynamic modes are the same order of magnitude as those for rotor blade flapping.

\subsubsection{Empennage Modeling and Main Rotor Interference}

Modeling of the horizontal and vertical stabilizers are essential for correct forward flight characteristics, and the importance of main rotor induced velocity interaction with these surfaces is well documented.

The wind tunnel tests of reference [20] show that loads produced at the horizontal stabilizer are directly related to impingement of main rotor downwash. The loads on the horizontal stabilizer are smaller at low speeds and also at high speeds when compared to the range of speeds that cause a direct impingement of rotor downwash upon the horizontal stabilizer. These tests also show that the main rotor downwash directly influences the thrust production capability of the tail rotor. Studies carried out at the Bell Helicopter Company ${ }^{[21]}$ show that the aerodynimic interaction between tail rotor and vertical fin 
can significantly influence a helicopter's low speed yaw response. This reference outlines formulas which may be used to account for the interference encountered. They also suggest that main rotor downwash may effect the vertical fin-tail rotor interaction. In reference $[19]$, the main rotor downwash and sidewash affects upon the surfaces of the fuselage are accounted for. They conclude that the static influences of the rotor wake on the tail rotor and fixed tail surfaces are the most important factor for forward flight dynamical analysis after the basic configuration modeling.

\subsection{Reference Frames}

The body-fixed fuselage coordinate system ( F) has its origin at the aircraft centre of gravity and is parallel to the fuselage reference line coordinate system (FRL). All specifications of the Bell 205 are given in terms of the body fixed FRL system which is scaled in terms of waterline (WL), fuselage station (FS), and butt line (BL). The body fixed reference frames used are shown in 2.1. The orientation of the $F$ coordinate system with respect to an earth system (assumed inertial) can be defined by the Euler angles $\Phi, \Theta$, and $\Psi$ as shown is figure 2.2. The transformation of a vector from the earth axes (I) to the $F$ axes can be defined by the following transformation matrix:

$$
\begin{aligned}
T_{F I} & =\left[\begin{array}{ccc}
C \Psi C \Theta & S \Psi C \Theta & -S \Theta \\
-S \Psi C \Phi+C \Psi S \Theta S \Phi & C \Psi C \Phi+S \Psi S \Theta S \Phi & C \Theta S \Phi \\
S \Psi S \Phi+C \Psi S \Theta C \Phi & -C \Psi S \Phi+S \Psi S \Theta C \Phi & C \Theta C \Phi
\end{array}\right] \\
C & \rightarrow \cos \\
S & \rightarrow \sin
\end{aligned}
$$

where the subscript $F I$ is used to signify that the transformation matrix $T_{F I}$ operates on vectors in the I frame and translates them to the $F$ frame.

It is also necessary to locate each blade element with respect to the $F$ axes. To do this, several intermediate axes are defined: Shaft (S), Rotor plane (R), Hub (H), and supplementary Blade $\left(B^{\prime}\right)$. Of these systems, only the shaft system is body fixed; the others are blade fixed and thus rotate with the blade.

The origin of the shaft coordinate system is at the flapping hinge and is defined by $\hat{z}_{s}$ pointing upward along the shaft, $\dot{x}_{s}$ pointing aft, and $\hat{y}_{s}$ starboard. Figure 2.3 shows the orientation of the shaft system with respect to the blade fixed systems. The rotor plane 


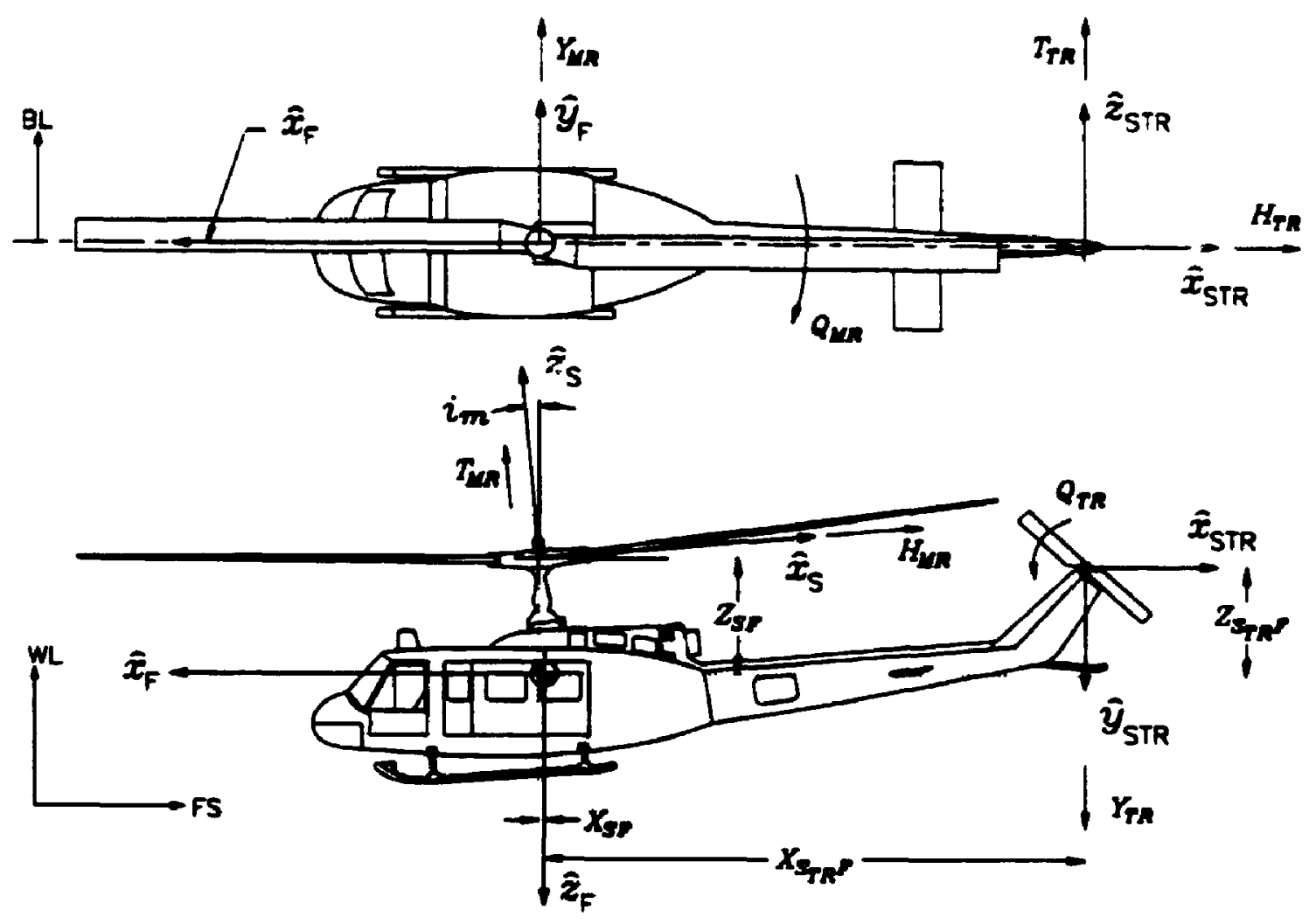

Figure 2.1: Body fixed axes: Fuselage(F), Shaft(S), and Shaf' $\cdot$ Tail-Rotor(STR).

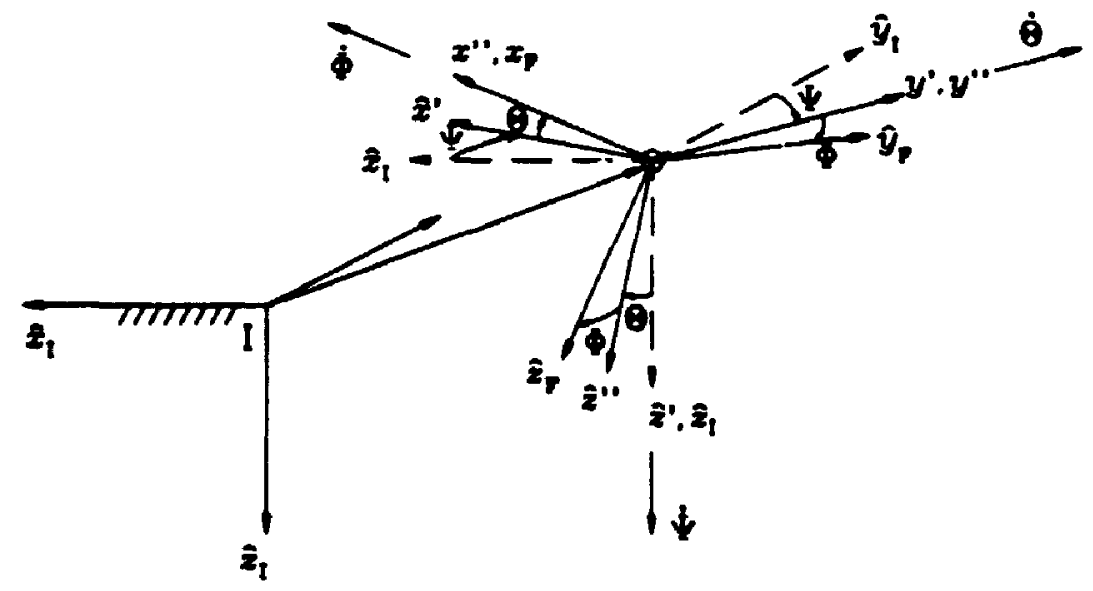

Figure 2.2: Inertial frame to fuselage frame transformation 


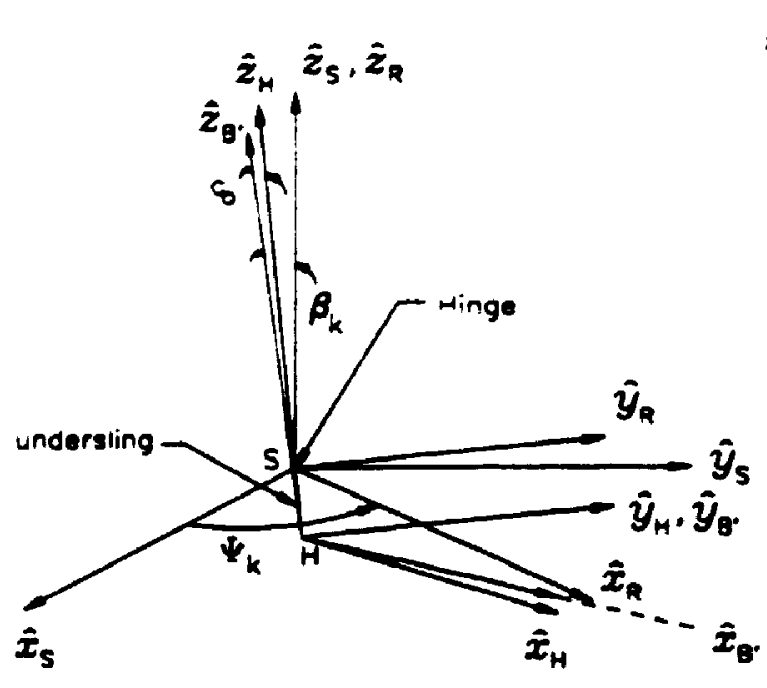

(a)
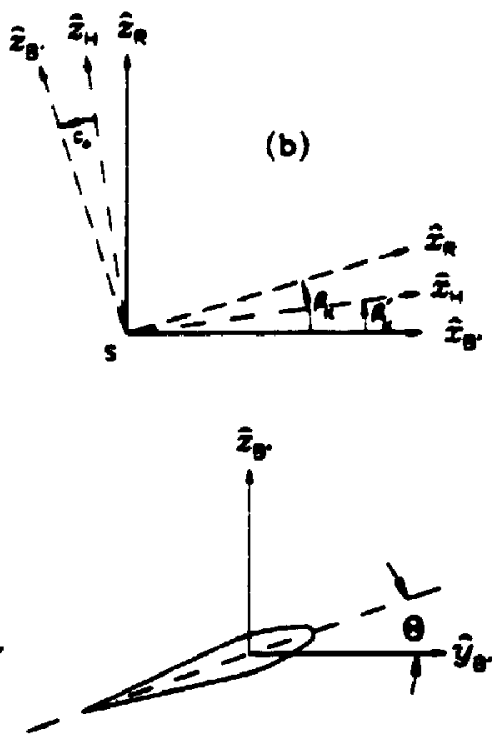

(c)

Figure 2.3: Blade axes systems

coordinate system is obtained by rotating the shaft axes about $\bar{z}_{s}$ by an angle of $i k$. This rotates $\dot{x}_{s}$ into a plane which contains the blades and as a result the angle $\psi_{k}$ is referred to as the blade azimuth. Two operations are required to translate system $\mathbf{R}$ to system $B^{\prime}$. First. a rotation about $\left(-\bar{y}_{R}\right)$ by the flapping angle $\beta_{k}$ to take $\hat{z}_{s}$ into $\hat{z}_{B^{\prime}}$. Second, a translation $\left(-Z_{S H} \dot{z}_{B^{\prime}}\right)$ to define the new origin $B^{\prime}$ at the centre of the hub. Axus $\dot{x}_{B^{\prime}}$ now defines the quarter cord line of the blade, however, axis $\hat{y}_{B}$, only lies along the cord of the blade when the pitch angle is zero (fig. 2.3(c)). The same steps can be used to define the hub coordinate system if the angle $\beta_{k}^{\prime}=\beta_{k}-c_{o}$ is used in place of $\beta_{k}$. The angle $c_{o}$ is the built in precone of the blade hub assembly and is clarified in figure 2.5 .

In the work that follows some use is made of a hub-wind coordinate system which is the shaft coordinate system rotated about $\hat{z}_{s}$ until the relative wind lies in the $\dot{x}_{s} \cdot \hat{z}_{s}$ plane.

The transformation matrices used in developing the equations of motion are:

$$
T_{S F}=\left[\begin{array}{ccc}
-C i_{m} & 0 & -S i_{m} \\
0 & 1 & 0 \\
S i_{m} & 0 & -C i_{m}
\end{array}\right]
$$




$$
\begin{aligned}
T_{R S} & =\left[\begin{array}{ccc}
C L_{k} & S L_{k} & 0 \\
-S L_{k} & C L_{k} & 0 \\
0 & 0 & 1
\end{array}\right] \\
T_{B^{\prime} R} & =\left[\begin{array}{ccc}
C 3_{k} & 0 & S 3_{k} \\
0 & 1 & 0 \\
-S 3_{k} & 0 & C 3_{k}
\end{array}\right] \\
T_{H R} & =\left[\begin{array}{ccc}
C 3_{k}^{\prime} & 0 & S 3_{k}^{\prime} \\
0 & 1 & 0 \\
-S 3_{k}^{\prime} & 0 & C 3_{k}^{\prime}
\end{array}\right]
\end{aligned}
$$

where $i_{m}$ is the forward tilt of the main rotor shaft with respect to $\dot{\xi}_{F}$ (fig. 2.1).

\subsection{Main Rotor}

The main rotor equations which consist of a set describing the tip path plane dynamics and also a set describing the forces and moments produced by the main rotor are derived in a body-fixed coordinate system. The advantage of a body-fixed approach rather than

a wind-axis approach ${ }^{[22-25]}$ is that it avoids the hover simulation problems which occur in conventional models that are caused by large instantaneous wind-axis angle changes for small changes in body velocities ${ }^{[26]}$.

\subsubsection{Assumptions}

The following is a list of assumptions and model simplifications that are used to simplify the modeling task:

1. rigid rotor in bending and torsion.

2. reverse flow effects are not modelled (negligible for $\mu<0.25$ or 120 knots $^{[24]}$ ).

3. compressibility and stall effects are not modelled.

4. inflow angle and flapping angle are assumed to be small thus:

- $\sin \beta_{k} \approx \beta_{k}, \cos \beta_{k} \approx 1$

- $\sin \varphi \approx \varphi, \cos \varphi \approx 1$ 
j. quasi-steady strip theory is used to determine aerodynamic loads.

f. radial flow along the blade is assumed to have no effect on blade aerodynamics.

\subsubsection{Tip-Path-Plane Approximation}

The differential equations which describe the flapping dynamics of the blade are nonlinear and have periodic coefficients [25]. There is no known closed form solution but it must be periodic in the azimuth angle $\left(w_{k}\right)$. It is therefore possible to express the solution as a Fourier series:

$$
\begin{aligned}
3(t)= & a_{0}(t)-a_{1}(t) \cos \left(\psi_{k}\right)-b_{1}(t) \sin \left(\psi_{k}\right)-a_{2}(t) \cos \left(2 \psi_{k}\right)- \\
& b_{2}(t) \sin \left(2 \psi_{k}\right)-a_{3}(t) \cos \left(3 \psi_{k}\right)-b_{3}(t) \sin \left(3 \psi_{k}\right)-\ldots
\end{aligned}
$$

Bramwell ${ }^{[25]}$ and McLarty ${ }^{[27]}$ point out that the magnitude of the above coefficients decrease very rapidly with order of harmonic. The magnitude of the coefficients are roughly one tenth of the value of that of the lower harmonic $\left(b_{i+1}=\frac{1}{10} b_{i}\right)$. In addition, it is noted that the effect of the ignored blade bending modes are at least as great as the higher harmonics of the rigid blade mode. It is therefore common practice to drop all harmonics above the first one leaving:

$$
\beta(t)=a_{0}(t)-a_{1}(t) \cos \left(\psi_{k}\right)-b_{1}(t) \sin \left(\psi_{k}\right)
$$

It can be shown that the second harmonics are not present for a rigid teetering rotor and in fact only odd harmonics exist for this type of rotor ${ }^{[28,29]}$. Therefore the above approximation is a very good description of the rigid blade mode of the teetering rotor. The following notation for flapping angle will be used in this thesis:

$$
\begin{aligned}
& \dot{\beta}(t)=c_{0}-\theta_{R} \cos \left(\psi_{k}\right)-\phi_{R} \sin \left(\psi_{k}\right) \\
& \dot{\beta}(t)=-\left(\dot{\theta}_{R}+\phi_{R} \Omega\right) \cos \left(\psi_{k}\right)+\left(\theta_{R} \Omega-\dot{\phi}_{R}\right) \sin \left(\dot{\psi}_{k}\right) \\
& \ddot{j}(t)=\left(-\ddot{\theta}_{R}-2 \dot{\phi}_{R} \Omega+\theta_{R} \Omega^{2}\right) \cos \left(\psi_{k}\right)+\left(2 \dot{\theta}_{R} \Omega+\phi_{R} \Omega^{2}-\ddot{\phi}_{R}\right) \sin \left(\psi_{k}\right)
\end{aligned}
$$

Equation (2.1) shows that the frequency of rotor flapping is exactly one per revolution forcing the blade tips to carve out a plane during a revolution (fig. 2.4). This plane is called the tip-path-plane or rotor disk and its orientation can be described by two angles: $\theta_{R}$ the longitudinal flapping angle, and $\phi_{R}$ the lateral flapping angle. The average value of 


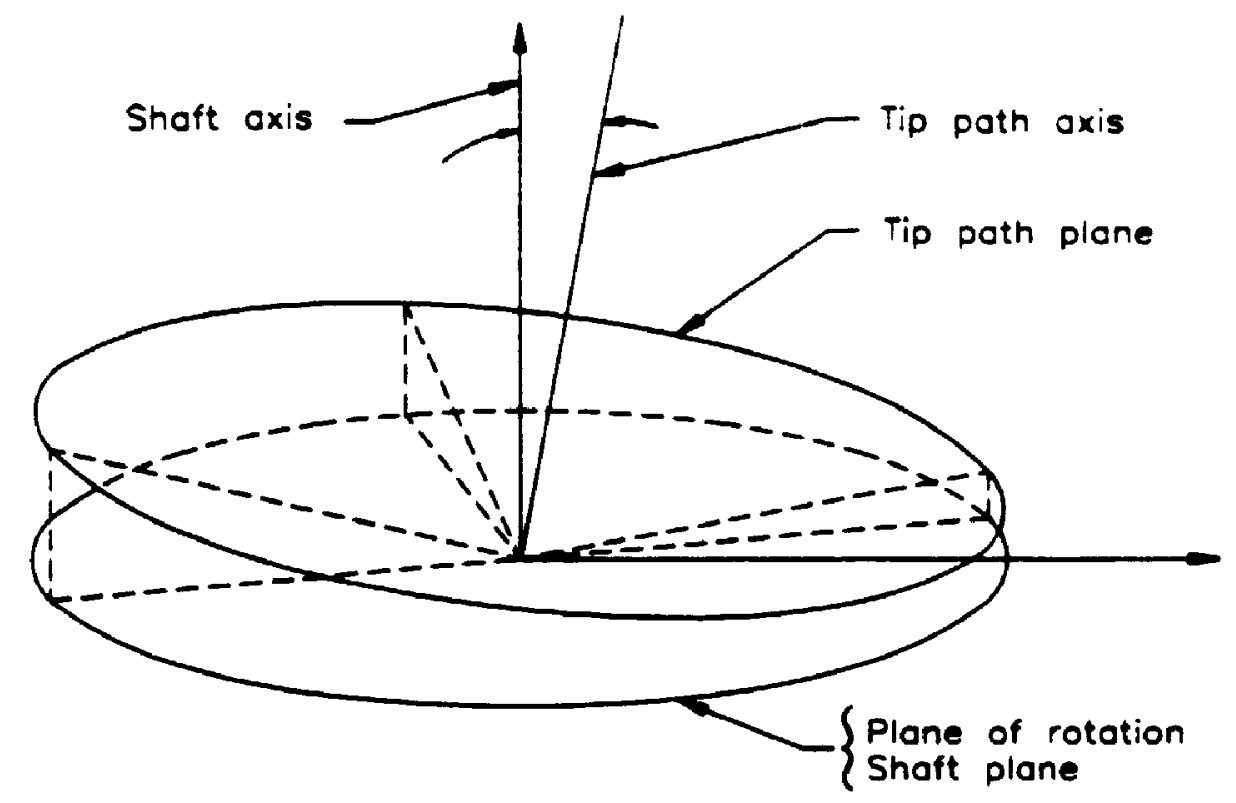

Figure 2.4: Tip path plane vs. shaft plane.

flapping represents a general upward tilt of all blades. For a teetering rotor this angle, $c_{0}$, is constant thus simplifying the analysis.

\subsubsection{Flapping Equations of Motion}

A Newtonian formulation is used to derive the equations of the rotor with the shaft axis as the base reference frame. Since the centre of gravity of the rotor system (two blades and hub assembly) is at the flapping hinge it is beneficial to study the complete rotor system as a unit rather than individual blades. The derivation as outlined below is straightforward but tedious. The symbolic processor of Mathematica is used to perform all algebraic manipulations and integrations that follow.

The sum of all external moments about the flapping hinge is equal to the time rate of change of the angular momentum about the hinge. Here, rotor fixed $\mathrm{H}^{\prime}$ axes are used which are the $\mathrm{H}$ axes shifted to have a centre at the flapping hinge (fig. 2.5). The angular momentum equation is thus:

$$
\vec{M}_{H^{\prime}}=\frac{d}{d t} \vec{H}=(\dot{\vec{H}})_{r}+\vec{\Xi}_{H^{\prime \prime} / t} \times \vec{H}
$$

where $د_{H^{\prime} / l}$ is the absolute angular velocity of the $H^{\prime}$ axes, $\vec{H}$ is the angular momentum, and $\vec{M}_{H^{\prime}}$ is the sum of all external moments acting on the rotor. The angular momentum 


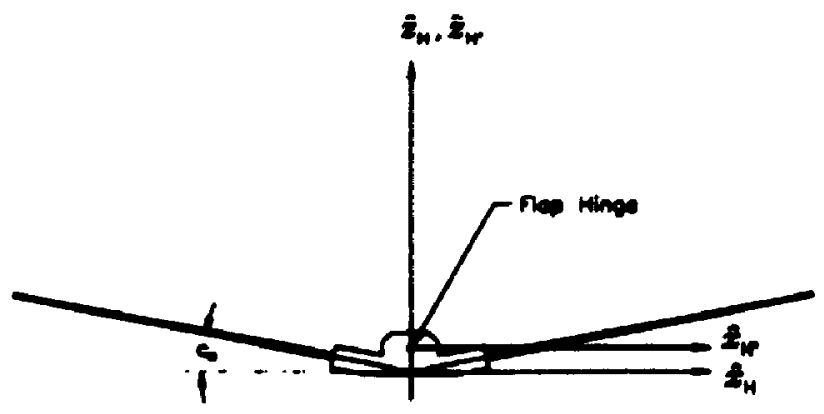

Figure 2.5: Hub fixed axes $H$ and $H^{\prime}$

of the rotor can be defined according to:

$$
\vec{H}=[I] \cdot \vec{\omega}_{H^{\prime} / I}
$$

where,

$$
\begin{aligned}
& {[I]=\left[\begin{array}{rrr}
I_{x x} & -I_{x y} & -I_{z x} \\
-I_{x y} & I_{y y} & -I_{y z} \\
-I_{z x} & -I_{y z} & I_{z z}
\end{array}\right]} \\
& I_{x y}=\int_{v} x y d m \\
& I_{x x}=\int_{v}\left(y^{2}+z^{2}\right) d m \\
& I_{y y}=\int_{v}\left(x^{2}+z^{2}\right) d m \\
& I_{x z}=\int_{v}\left(x^{2}+y^{2}\right) d m \\
& I_{x z}=I_{y z} \approx 0
\end{aligned}
$$

The inertias $I_{x z}$ and $I_{y z}$ are assumed negligible as the blade thickness is small compared to its span and cord. If $\omega_{H \prime \prime}$ is expressed as $\left(\omega_{x}, \omega_{y}, \omega_{z}\right)$, then equation (2.3) can be expanded to give:

$$
\left[\begin{array}{l}
M_{z} \\
M_{y} \\
M_{z}
\end{array}\right]_{H^{\prime}}=\left[\begin{array}{l}
I_{x z} \dot{\omega}_{z}+\left(I_{z z}-I_{y y}\right) \omega_{y} \omega_{z}-I_{x y}\left(\dot{\omega}_{y}-\omega_{x} \omega_{z}\right) \\
I_{y y} \dot{\omega}_{y}+\left(I_{x z}-I_{z z}\right) \omega_{z} \omega_{z}-I_{x y}\left(\dot{\omega}_{x}+\omega_{y} \omega_{z}\right) \\
I_{z z} \dot{\omega}_{z}+\left(I_{y y}-I_{x x}\right) \omega_{x} \omega_{y}-I_{x y}\left(\omega_{z}^{2}-\omega_{y}^{2}\right)
\end{array}\right]
$$

Since the flapping hinge is aligned with the $y$ axis of the $H^{\prime}$ coordinate system, only the y component of equation (2.4) is directly applicable to flapping dynamics.

The angular velocity of the rotor may be developed by considering the fuselage angular rates $(p, q, r)$ in the fuselage axes, rotor constant angular rate $\left(\Omega=d \Psi_{k} / d t\right)$ in the shaft 
axes, and flapping rate in the hub axes.

$$
\begin{aligned}
\vec{y}_{H^{\prime} / l} & =\{p . q, r\} \cdot\left\{\dot{x}_{F}, \dot{y}_{F}, \dot{z}_{F}\right\}+\Omega \dot{z}_{R}-\dot{j}_{k} \dot{y}_{H} \\
\vec{\omega}_{H^{\prime} / 1} & =T_{H R} \cdot T_{R S} \cdot T_{S F} \cdot{ }_{F} \vec{y}_{F / I}+T_{H R} \cdot \Omega \dot{j}_{R}-\dot{j}_{k} \dot{y}_{H}
\end{aligned}
$$

Substituting ${ }_{H} \overline{\vec{w}}_{H^{\prime \prime} / I}$, its component time derivative, equation (2.2), and the relation $. b_{k}^{\prime}=$ $\beta_{k}-c_{0}$ into equation (2.4) yields the flapping equation as a function of time. A change is made from differentiation with respect to time to differentiation with respect to rotor azimuth according to:

$$
\frac{d(\cdot)}{d t}=\frac{d(\cdot)}{d \psi_{k}} \frac{d \psi_{k}}{d t}=\Omega \frac{d(\cdot)}{d \psi_{k}}=\Omega(\cdot)
$$

where the tilde is used to denote differentiation with respect to azi uth. The resulting equation contains some higher order harmonic terms (appearing as powers of sine and cosine) which were previoussly explained to be negligible. In order to remove these terns, the trigonometric identit.es listed in Appendix $A$ are used to set a'l harmonics above one to zero. For example:

$$
\sin ^{3}(\psi) \equiv \frac{3 \sin (\psi)}{4}-\frac{\sin (3 \psi)}{4} \Rightarrow \frac{3 \sin (\psi)}{4}
$$

The resulting flapping equation is:

$$
\begin{aligned}
& \left(M_{y}\right)_{H^{\prime}}=\Omega^{2} \cos \psi_{k}\left\{\theta_{R}\left(-I_{x x z z}-I_{y y}+I_{x y} \tilde{\dot{\phi}} \sin i_{m}-I_{x y} \tilde{\dot{\psi}} \cos i_{m}\right)\right. \\
& +I_{y y} \tilde{\theta}_{R}+2 I_{y y} \bar{\phi}_{R}+2 I_{x y} \tilde{\theta}+I_{y y} \tilde{\theta}+I_{x y} \bar{\phi} \cos i_{m} \\
& \left.+\left(I_{y y}-I_{x x z s}\right)\left(\ddot{\phi} \cos i_{m}+\tilde{\psi} \sin i_{m}\right)\right\} \\
& +\Omega^{2} \sin \psi_{k}\left\{\phi_{n}\left(-I_{x x z z}-I_{y y}-I_{x y} \tilde{\tilde{\psi}} \cos i_{m}+I_{x y} \tilde{\dot{\phi}} \sin i_{m}\right)\right. \\
& +I_{y y} \tilde{\phi}_{k}-2 I_{y y} \dot{\phi}_{k}-I_{x y} \tilde{\tilde{\theta}}+I_{y y} \tilde{\phi} \cos i_{m}-2 I_{x y} \dot{\phi} \cos i_{m} \\
& \left.-\left(I_{y y}-I_{x x z z}\right) \tilde{\theta}-2 I_{x y} \tilde{\psi} \sin i_{m}+I_{y y} \overline{\bar{\psi}} \sin i_{m}\right\}+ \text { h.o.t }
\end{aligned}
$$

where $I_{x x z s}=I_{x z}-I_{z z}$, and $\left(M_{y}\right)_{H^{\prime}}$ is the sum of all external moments that contribute to flapping which may be derived by considering the aerodynamic loads produced by the rotor.

\section{Aerodynamic Flapping Moment}

The aerodynamic moment is determined by defining the incremental lift and drag acting on a blade element and then integrating from the blade root to blade tip. This requires 


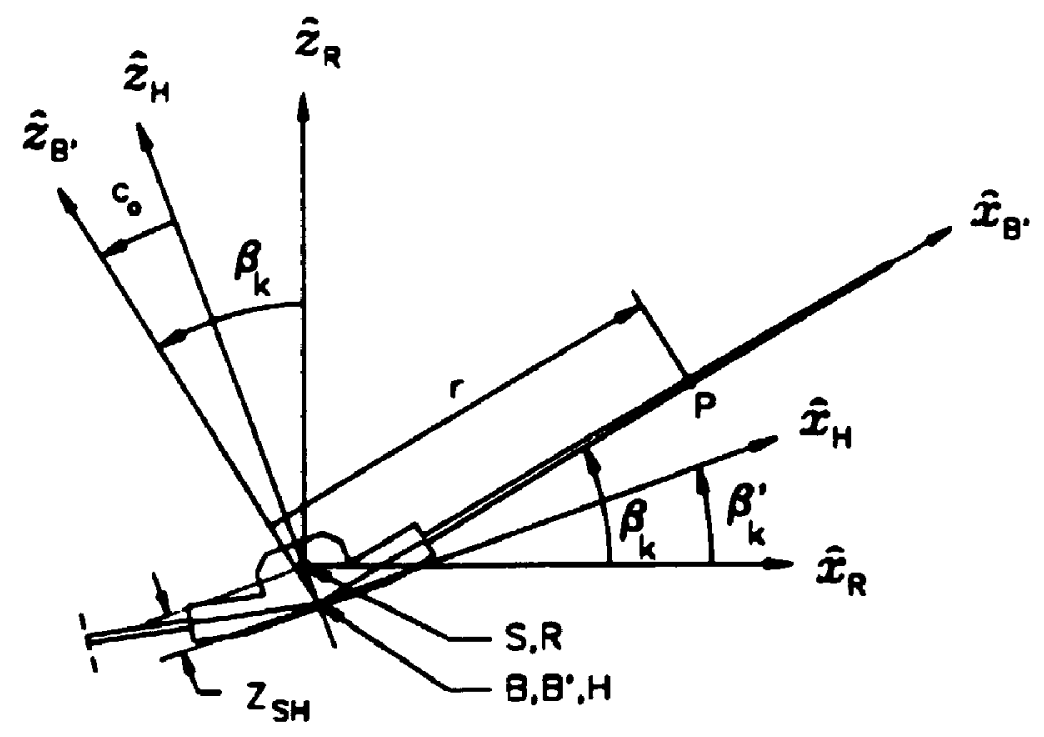

Figure 2.6: Geometry of flapping blade.

an expression for the blade element relative-wind velocity as a function of radial station and azimuth. In doing this the common assumption will be made that the radial velocity at the blade element (in the direction of $\dot{x}_{B^{\prime}}$ ) has no effect on lift and drag of the element.

Consider an element (P) on a blade as shown in figure 2.6. Its velocity in vector form is:

$$
{ }_{B}, \vec{v}_{P}={ }_{B}, \vec{v}_{S}+{ }_{B^{\prime}} \vec{v}_{P / S}=\left\{U_{R}, U_{T}, U_{P}\right\}_{B}
$$

where $U_{R}, U_{T}, U_{P}$ are the blade element velocities in the radial, tangential, and normal directions respectively. The first component, $\theta^{\prime} \vec{v}_{S}$, is the velocity of the flapping hinge and is defined by:

$$
{ }_{B^{\prime}} \vec{v}_{S}=T_{B^{\prime} R} \cdot T_{R S} \cdot T_{S F} \cdot \vec{v}_{S}
$$

where,

$$
\begin{aligned}
& \vec{v}_{s}=\vec{v}_{F}+\vec{w}_{F / I} \times \vec{r}_{s / F}=\text { velocity of hinge (in } F \text { system) } \\
& \vec{v}_{F}=u_{F} \hat{x}_{F}+v_{F} \hat{y}_{F}+w_{F} \hat{z}_{F}=\text { c.g. velocity of helucopter } \\
& \left.\vec{r}_{S / F}=X_{S F} \hat{x}_{F}-Z_{S F} \hat{z}_{F}=\text { position vector (F to } S\right) \\
& \vec{\omega}_{r / l}=p \dot{x}_{r}+q \dot{y}_{r}+r \dot{\xi}_{r}=\text { fuselage angular rate }
\end{aligned}
$$

The term ${ }_{B}, \vec{v}_{p / s}$ in equation (2.8) is the relative velocity of point $\mathrm{P}$ to the flapping hinge. 
It may be described by:

$$
\vec{v}_{P / s}=\vec{j}_{B^{\prime} / 1} \times \vec{r}_{P / S}
$$

where $\vec{J}_{B^{\prime} / l}$ is the absolute angular velocity of the blade and $\vec{r}_{P / S}$ is the position vector $S$ to $P$. The former translated to the $B^{\prime}$ system is:

$$
{ }_{B^{\prime}} \bar{B}_{B^{\prime} / I}=T_{B^{\prime} R} \cdot T_{R S} \cdot T_{S F, \bar{F}_{F / I}}+T_{B^{\prime} R} \cdot\left\{0-\dot{\beta}_{k} \Omega\right\}^{T}
$$

Figure 2.6 can be used to derive the position vector $\vec{r}_{P / S}$ :

$$
{ }_{B^{\prime}} \vec{r}_{P / S}=\left(r-Z_{S H} \sin \left(c_{o}\right)\right) \dot{x}_{B^{\prime}}-Z_{S H} \cos \left(c_{o}\right) \dot{z}_{B^{\prime}}
$$

where,

$$
\begin{aligned}
Z_{s H} & =\text { hub undersling }(f g \cdot 2.6) \\
r & =\text { radius from } B^{\prime} \text { to } P
\end{aligned}
$$

Equation (2.8) may now be solved for $U_{T}$ and $U_{P}$ :

$$
\begin{aligned}
U_{T}= & \left(\Omega-\dot{\psi} \cos i_{m}+\dot{\phi} \sin i_{m}\right)\left(r+Z_{s H} \cos c_{o} \beta_{k}-Z_{s H} \sin c_{o}\right)+ \\
& \cos \psi_{k}\left\{v_{F}+\dot{\psi} X_{s F}+\dot{\phi} Z_{s F}+\left(\dot{\psi} \cos i_{m}+\dot{\phi} \cos i_{m}\right)\left(r \beta_{k}-\right.\right. \\
& \left.\left.Z_{s H} \cos c_{o}-Z_{s H} \beta_{k} \sin c_{o}\right)\right\}+ \\
& \sin \psi_{k}\left\{u_{F} \cos i_{m}+w_{F} \sin i_{m}+\dot{\theta}\left(-r \beta_{k}+\right.\right. \\
& \left.\left.Z_{s H} \cos c_{o}+Z_{s H} \beta_{k} \sin c_{o}-Z_{s F} \cos i_{m}-X_{s F} \sin i_{m}\right)\right\} \\
U_{P}= & \dot{\beta}_{k}\left(r-Z_{s H} \sin c_{o}\right)+\dot{\theta}\left(X_{s F} \cos i_{m}-Z_{s F} \sin i_{m}\right)- \\
& w_{F} \cos i_{m}+u_{F} \sin i_{m}+ \\
& \cos \psi_{k}\left\{\dot{\theta}\left(-r-Z_{s F} \beta_{k} \cos i_{m}+Z_{s H} \sin c_{o}-X_{s F} \beta_{k} \sin i_{m}\right)+\right. \\
& \left.u_{F} \beta_{k} \cos i_{m}+w_{F} \beta_{k} \sin i_{m}\right\}+ \\
& \sin \psi_{k}\left\{\dot{\phi}\left(-r \cos i_{m}-Z_{s F} \beta_{k}+Z_{s H} \cos i_{m} \sin c_{o}\right)+\right. \\
& \left.\dot{\psi}\left(-r \sin i_{m}-X_{s F} \beta_{k}+Z_{s H} \sin c_{o} \sin i_{m}\right)-v_{F} \beta_{k}\right\}
\end{aligned}
$$

where the small angle approximation has been used for $\beta_{k}$, and $U_{R}$ has been omitted since it is assumed to have negligible effect upon the blade aerodynamics.

In order to calculate aerodynamic loads, we require the relative wind velocities as seen by a blade element. Thus, the blade element velocities defined in equation (2.9) must be modified by reversing their directions (graphically, fig. 2.7) and including the induced velocity generated by the main rotor, $v_{i}(r, \psi)$. It is also convenient to non-dimensionalise 
equation (2.9) by dividing by the tip speed $(\Omega R)$ and changing from differentiation with respect to time to differentiation with respect to rotor azimuth (equation(2.6)). The overbar is used to denote a non-dimensional quantity; distances are converted with rotor radius ( $R$ ), velocities are converted with rotor-tip-speed, and angular rates are converted with rotor angular rate $(\Omega)$. The resulting non-dimensional form of $U_{T}$ and $U_{P}$ are:

$$
\begin{aligned}
& \bar{U}_{T}=\left(1-\bar{\psi} \cos i_{m}+\tilde{\phi} \sin i_{m}\right)\left(x+\bar{Z}_{S H} \beta_{k} \cos c_{o}-\bar{Z}_{S H} \sin c_{o}\right)+ \\
& \cos \psi_{k}\left\{\bar{v}_{r}+\bar{\psi} \bar{X}_{s F}+\bar{\phi} \bar{Z}_{s F}+\left(\bar{\psi} \cos i_{m}+\bar{\phi} \cos i_{m}\right)\left(x \beta_{k}-\right.\right. \\
& \left.\left.\bar{Z}_{S H} \cos c_{o}-\bar{Z}_{S H} \beta_{k} \sin c_{o}\right)\right\}+ \\
& \sin \psi_{k}\left\{\bar{\psi}_{r} \cos i_{m}+\bar{w}_{F} \sin i_{m}+\tilde{\theta}\left(-x \beta_{k}+\right.\right. \\
& \left.\left.\bar{Z}_{S H} \cos c_{o}+\bar{Z}_{S H} \beta_{k} \sin c_{o}-\bar{Z}_{S F} \cos i_{m}-\bar{X}_{S F} \sin i_{m}\right)\right\} \\
& \bar{U}_{P}=\bar{\beta}_{k}\left(x-\bar{Z}_{S H} \sin c_{o}\right)+\dot{\theta}\left(\bar{X}_{S F} \cos i_{m}-\bar{Z}_{S F} \sin i_{m}\right)- \\
& \bar{w}_{F} \cos i_{m}+\bar{u}_{f} \sin i_{m}+\lambda_{0}+ \\
& \cos \psi_{k}\left\{\bar{\theta}\left(-x-\bar{Z}_{S F} \beta_{k} \cos i_{m}+\bar{Z}_{S H} \sin c o-\bar{X}_{S F} \beta_{k} \sin i_{m}\right)+\right. \\
& \left.\bar{u}_{m} \beta_{k} \cos i_{m}+\bar{w}_{F} \beta_{k} \sin i_{m}+\lambda_{c}\right\}+ \\
& \sin \psi_{k}\left\{\tilde{\phi}\left(-x \cos i_{m}-\bar{Z}_{s F} \beta_{k}+\bar{Z}_{s H} \cos i_{m} \sin c_{o}\right)+\right. \\
& \left.\bar{\psi}\left(-x \sin i_{m}-\bar{X}_{s F} \beta_{k}+\bar{Z}_{s H} \sin c_{o} \sin i_{m}\right)-\bar{v}_{F} \beta_{k}+\lambda_{s}\right\}
\end{aligned}
$$

where the inflow variation $\bar{v}_{i}\left(r, \psi_{k}\right)=\lambda_{0}+\lambda_{s} \sin \psi_{k}+\lambda_{c} \cos \psi_{k}$ has been introduced and $x$ is the non-dimensional span distance $r / R$. The form of the inflow variation will be discussed in more detail in section 2.3.5.

The flow at the blade element is shown in figure 2.7 along with the differential lift, $d \mathcal{L}$, and profile drag, $d \mathcal{D}$, generated by the blade element. The component of the total force, $d \mathcal{F}$, that contributes to flapping moment is aligned with $\hat{z}_{B^{\prime}}$, and can be expressed for small inflow angle $(\varphi)$ as:

$$
d L_{a}=d \mathcal{C}-d D \varphi
$$

The quasi-static lift and profile drag per unit span on an airfoil are:

$$
\begin{aligned}
\mathcal{L} & =\frac{1}{2} \rho a c V^{2} \alpha \\
\mathcal{D} & =\frac{1}{2} \rho c c_{d} V^{2}
\end{aligned}
$$

where,

$$
\rho=\text { air density }\left(\mathrm{kg} / \mathrm{m}^{3}\right)
$$




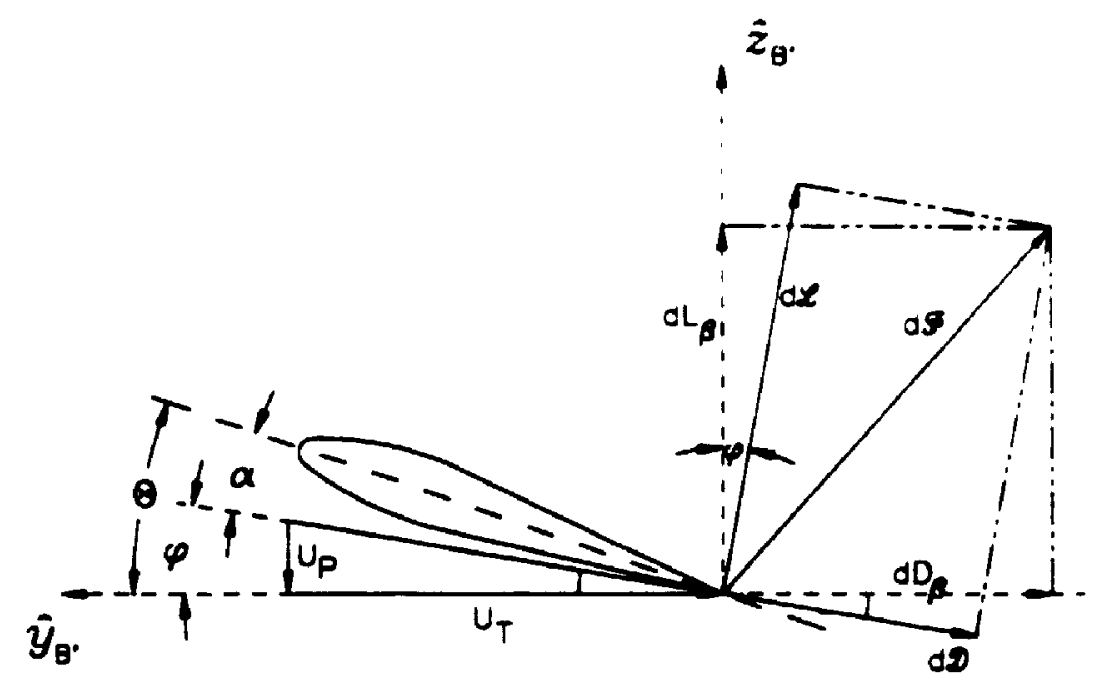

Figure 2.7: Airflow geometry at blade element

$$
\begin{aligned}
a & =\text { lift curve slope }(1 / \mathrm{rad}) \\
c & =\text { blade cord }(\mathrm{m}) \\
V & =\sqrt{U_{P}^{2}+U_{T}^{2}}(\mathrm{~m} / \mathrm{s}) \\
c_{d} & =\text { drag coefficient } \\
\alpha & =\text { angle of attack of blade element } \\
& =\theta-\varphi \approx \theta-\tan (\varphi)=\theta-\frac{U_{P}}{U_{T}} \\
\theta & =\text { blade pitch angle measured from hub plane }(\mathrm{rad})
\end{aligned}
$$

In approximating the drag coefficient, the induced drag contribution is ignored in order to simplify the equations and allow a closed form solution ${ }^{2}$. The profile drag contribution is approximated according to:

$$
c_{d}=c_{d_{0}}+c_{d_{1}}\left(\frac{6 C_{T}}{a \sigma}\right)+c_{d_{2}}\left(\frac{6 C_{T}}{a \sigma}\right)^{2}
$$

where $C_{T}$ is the rotor thrust coefficient $\frac{T}{\rho \pi \Omega^{2} R^{T}}$, and $c_{d_{0}}, c_{d_{1}}$, and $c_{d_{2}}$ are chosen to match the blade section characteristics and $\frac{6 C_{T}}{a \sigma}$ is the average rotor blade angle of attack ${ }^{[24]}$. In the above expression, the blade pitch has been used which may be defined as:

$$
\theta=\theta_{0}+\theta_{t} \frac{r}{R}+A_{c} \cos \left(\psi_{k}\right)+A_{0} \sin \left(\psi_{k}\right)
$$

\footnotetext{
${ }^{2}$ the induced drag is small when compared to profile drag because of the high blade appect ratio
} 
Whare $\theta_{\text {, is }}$ ise blade-root collective pitch measured from the hub plane. $\theta_{\text {, }}$ is the linear iwist of the blade tip with respect to blade root. and $t_{0}$ and $t_{\text {s }}$ are the lateral and Iongitudinal ryclic pitch. respectively. measured from the hub plane and in the shaft sistem.

Equation $(2.11)$ in non-dimensional form becomes:

$$
d L_{3}\left(r . L_{k} k\right)=\frac{1}{2} \rho c \Omega^{2} R^{3}\left[a\left(\bar{C}_{T}^{2} \theta-\bar{l}_{T} \bar{l}_{P}\right)-c_{1} \bar{C}_{T} \bar{C}_{P}\right] d x
$$

where $\tau_{p} \ll 1$ has been used to simplify the equation. In calculating the flappirg moment both blades must be considered, one blade at azimuth $u_{k} k$ and the other at $\dot{u}_{k}+\pi$. The flapping moment is thus:

$$
\begin{aligned}
& M_{s}=R \int_{x_{0}}^{B P} x\left[d L_{g}\left(r, \iota_{k}\right)-d L_{g}\left(r, \iota_{k}+\pi\right)\right] \quad\left\{-\hat{y}_{B^{\prime}}\right\} \\
& =\frac{1}{2} \rho c R^{4} \Omega^{2} \int_{r_{0}}^{B P} x\left[a\left(\bar{U}_{T_{1}}^{2} \theta_{1}-\bar{C}_{T_{2}}^{2} \theta_{2}-\bar{U}_{T_{1}} \bar{l}_{P_{1}}+\bar{U}_{T_{2}} \bar{l}_{P_{2}}\right)\right. \\
& \left.+c_{d}\left(\bar{U}_{T_{2}} \bar{U}_{P_{2}}-\bar{U}_{T_{1}} \bar{U}_{P_{1}}\right)\right] d x
\end{aligned}
$$

where the subscripts $(\cdot)_{1}$ and $\left(\cdot h_{2}\right.$ are used to denote values for blade one and two and it has been assumed that $\left(r-Z_{S H} \sin c_{o}\right) \approx \cdots$. The velocity and pitch of blade one $\left(U_{T_{1}} \cdot r_{P_{1}}, \theta_{1}\right)$ are obtained from equations $(2.10)$ and (2.13) respectively. The ve ocity and pitch of blade two may be derived from that of blade one according to:

$$
(\cdot)_{2}=\left(\cdot h \rightarrow \left\{\begin{array}{l}
\dot{\psi}_{k}-\psi_{k}+\pi \\
\dot{i}-2 c_{o}-3_{k} \\
\tilde{3}_{k} \rightarrow-\overline{3}_{k}
\end{array}\right.\right.
$$

The root cutout factor, $x_{0}$, takes into account the fact that the blades are installed at a distance of $r=x_{0} R$ from the centre of rotation. Also, due to the air flow around the blade tips the lift of the rotor blade goes to zero at $r=R$ and also starts to fall off at some distance inboard of the tip. To account for this "tip-loss", the blade lift is integrated only from $x_{0}$ to $B P$. A well known approximate relation for the tip-loss factor, $B P$, as developed by Prandtl and Betz(1927) is:

$$
B P=1-\frac{\sqrt{2 C_{T}}}{b}
$$

where.

$$
\begin{aligned}
& C_{T}=\text { rotor thrust coefficient } \\
& b=\text { No. of blades }
\end{aligned}
$$


Before $(2.15)$ can be integrated expressions (2.2) are used to replace blade flapping anglts and rate. It is once again necessary to use the trigonometric identities from appendix $A$ to reduce all harmonics to the first order.

The aerodynamic moment term found in equation (2.i) may now be replaced by tho result of equation (2.15) noting that $M_{y_{H^{\prime}}}=-M_{s}$. The result is an identity of the form:

$$
\left(\cdots k_{\text {onot }}+(\cdots)_{\text {oc }} \cos \left(\psi_{k}\right)+(\cdots)_{\text {on }} \sin \left(\psi_{k}\right)=0\right.
$$

that must be satisfied for all $\psi_{k}$. This results in three equations:

$$
\begin{aligned}
& (\cdots)_{\text {onse }}=0 \\
& (\cdots)_{\text {ose }}=0 \\
& (\cdots)_{\text {ie }}=0
\end{aligned}
$$

For an articulated rotor, expression (2.17) is a differential equation describing the coning degree of freedom. Since a teetering rotor has a constant coning angle, this relation is not used. Expressions (2.18) and (2.19) are differential equations describing the longitudinal and lateral flapping degrees of freedom respectively. If no simplifications are made, these expressions are very large (over 1000 terms) and cpu intensive in a computer simulation, thus, some simplifying assumptions are made before their use.

Now that the rotor degrees of freedom have been defined, it is possible to determine the loads generated by the rotor.

\subsubsection{Main Rotor Aerodynamic Loads}

In this section the aerodynamic forces and torque generated by the rotor will be derived. It is common practice to call the generated forces that are aligned with the main rotor shaft $\left(i_{s}\right)$, thrust. The other components of the generated force lie in the rotor plane, and are generally called rotor drag, and may be split into components and $\hat{y}_{s}$ (rotor $\mathrm{Y}$-force). The strictly proper definitions of $\mathrm{H} / \mathrm{Y}$-force are in the hub-wind coordinate system. Therefore, the H/Y-force derived in this thesis will only match other so urces when the relative wind is in the longitudinal plane of the helicopter and pointing aft. However, the notation adopted here lends itself to a computer simulation of the full helicopter, where as the classical notation is generally used for rotor-only analysis. 


\section{Thrust}

The blade elemental forces were shown in figure 2.7 and the component of the total force along the $\dot{z}_{B^{\prime}}$ axis was given by equation (2.11). The component of this force contributing to thrust is $d L_{3} \cos 3 \approx d L_{3}$ and is shown in figure 2.8. The total thrust is obtained by integrating the differential thrust. $d L_{3}$, along the blade:

$$
T\left(L_{k}\right)=\int_{\text {bladel }} d L_{3} d r+\int_{\text {blade2 }} d L_{3} d r
$$

This equation describes the total thrust produced by the rotor as a function of azimuth. It is highly desirable to remove any dependence on $v_{k}$ if possible. since it is not usually measured on the helicopter and it adds complexity. It is possible to define an average thrust per blade by integrating the thrust produced by a blade $T_{1}\left(\psi_{k}\right)$ around the azimuth and dividing by $2 \pi$, thus removing an explicit dependence on azimuth. Since the average thrust produced by each blade must be equal, an additional simplification can be made by considering only one blade and doubling the resulting thrust. The total average thrust is now:

$$
\begin{aligned}
T_{M R}= & \frac{1}{\pi} \int_{0}^{2 \pi} \int_{b l a d e_{1}} d L_{g} d r d w_{k} \\
= & \frac{1}{2 \pi} \rho c \Omega^{2} R^{3} \int_{0}^{2 \pi}\left[\int_{I 0}^{B P} a\left(\bar{C}_{T}^{2} \theta-\bar{C}_{T} \bar{C}_{P}\right) d x\right. \\
& \left.-\int_{0}^{1} c_{1} \bar{U}_{T} \bar{U}_{P} d x\right] d \psi_{k}
\end{aligned}
$$

where equation (2.14) has been used for $d L_{s}$ as before but the drag terms have been integrated from the centre of rotation $(x=0)$ to the normalized blade tip $(x=1)$. In additic $u$, before integrating, relations $(2.2)$ and $(2.13)$ are used to replace 3 and $\theta$.

\section{Rotor Drag}

The aerodynamic loads contributing to the $H$ and $Y$ forces are shown in figure 2.8 where:

$$
\begin{aligned}
d D_{3} & =d \mathcal{L} \sin \varphi+d \mathcal{D} \cos \varphi \\
& \approx d \mathcal{L} \varphi+d \mathcal{D}
\end{aligned}
$$

The diffecential $\mathbf{H}$-force and $\mathrm{Y}$-force are:

$$
d H_{M R}=d D_{g} \sin \psi_{k}-3 d L_{g} \cos \psi_{k}
$$




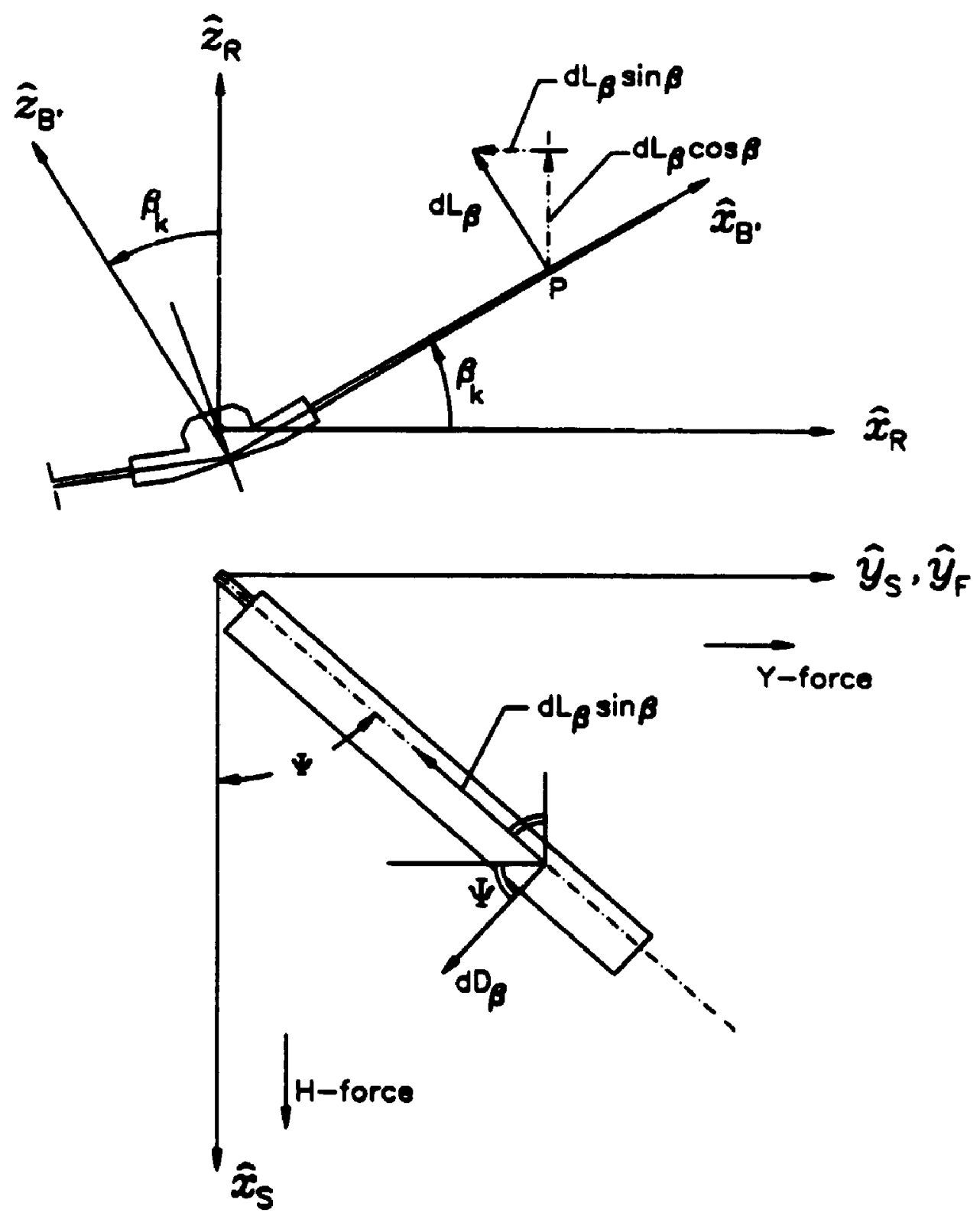

Figure 2.8: Main rotor aerodynamic loads. 


$$
\begin{aligned}
& =d \mathcal{L}\left(\varphi \cos \psi_{k}-3 \cos \psi_{k}\right)+d \mathcal{D}(\sin \psi_{k}+\overbrace{\varphi 3 \cos \psi_{k}}^{\approx 0}) \\
d Y_{M R} & =-d D_{g} \cos \psi_{k}-d L_{s}, 3 \sin \psi_{k} \\
& =d \mathcal{L}\left(-\varphi \cos \psi_{k}-3 \sin \psi_{k}\right)+d \mathcal{D}(-\cos \psi_{k}+\overbrace{-3 \sin \psi_{k}}^{\approx 0})
\end{aligned}
$$

As with thrust, the dependence of these forces on azimuth will be removed by considering their averages around the azimuth:

$$
\begin{aligned}
H_{M R}= & \frac{1}{2 \pi} \rho c \Omega^{2} R^{3} \int_{0}^{2 \pi}\left[\int_{x o}^{B P} a\left(\bar{U}_{T}^{2} \theta-\bar{U}_{T} \bar{U}_{P}\right)\left(\frac{\bar{U}_{P}}{\bar{U}_{T}} \sin \psi_{k}-\beta \cos \psi_{k}\right) d x\right. \\
& \left.+\int_{0}^{1} c_{d}\left(\bar{U}_{T}^{2}+\bar{U}_{P}^{2}\right) \sin \psi_{k} d x\right] d \psi_{k} \\
Y_{M R}= & \frac{1}{2 \pi} \rho c \Omega^{2} R^{3} \int_{0}^{2 \pi}\left[-\int_{x o}^{B P} a\left(\bar{U}_{T}^{2} \theta-\bar{U}_{T} \bar{U}_{P}\right)\left(\frac{\bar{U}_{P}}{\bar{U}_{T}} \cos \psi_{k}+\beta \sin \psi_{k}\right) d x\right. \\
& \left.-\int_{0}^{1} c_{d}\left(\bar{U}_{T}^{2}+\bar{U}_{P}^{2}\right) \cos \psi_{k} d x\right] d \psi_{k}
\end{aligned}
$$

\section{Torque}

The aerodynamic torque produced by the rotor will be considered positive in the $-\dot{z}_{S}$ direction. The differential torque from figure 2.8 is:

$$
d Q_{M R}=r d D_{\theta}=r(d \mathcal{L} \varphi+d \mathcal{D})
$$

Thus the average torque becomes:

$$
\begin{aligned}
Q_{N R}= & \frac{1}{2 \pi} \rho c \Omega^{2} R^{4} \int_{0}^{2 \pi}\left[\int_{x 0}^{B P} x\left[a\left(\bar{U}_{T} \bar{U}_{P}\right)\left(\theta-\frac{\bar{U}_{P}}{\bar{U}_{T}}\right)\right] d x\right. \\
& \left.+\int_{0}^{1} x c_{d}\left(\bar{U}_{\tau}^{2}+\bar{U}_{P}^{2}\right) d x\right] d \psi_{k}
\end{aligned}
$$

\section{Main rotor Contribution to the Total Forces and Moments}

The orientation of the forces and moments produced by the main rotor are shown in figure 2.1. It follows that:

$$
\begin{aligned}
& F_{I_{M R}}=-H_{M R} \cos i_{m}+T_{M R} \sin i_{m} \\
& F_{Y_{M R}}=Y_{M R}
\end{aligned}
$$




$$
\begin{aligned}
& F_{Y_{Y R}}=-H_{Y R} \sin i_{m}-I_{Y R} \cos i_{m} \\
& M_{Y_{M R}}=Y_{M R} Z_{S F}-Q_{M R} \sin i_{m} \\
& M_{Y_{M R}}=H_{M R}\left(Z_{\xi F} \cos \imath_{m}+X_{F F} \sin \imath_{m}\right)+\Gamma_{Y R}\left(X_{F F}\left(\cos l_{m}-Z_{F F} \sin l_{m}\right)\right. \\
& H_{s_{M R}}=Q_{M R} \cos \imath_{m}+Y_{M R} \mathbf{K}_{s F}
\end{aligned}
$$

where $F_{Y_{M R}} . F_{Y_{M R}}$, and $F_{s_{M R}}$ are the longitudinal. lateral. and vertical forces respectively in the fuselage coordinate system and $M_{I_{M R}}, M_{Y_{M R}}$, and $M_{z_{M R}}$ are the rolling. pitching. and yawing moments respectively.

\subsubsection{Dynamic Inflow}

The variation of induced inflow over the rotor disk is defined in a tip-path-plane(TPP). wind coordinate system by:

$$
\lambda\left(r . \nu_{w^{\prime}}\right)=\nu_{0}+\nu_{s} \frac{r}{R} \sin \psi_{w}+\nu_{c} \frac{r}{R} \cos \psi_{w}
$$

where $\psi_{w}$ is the rotor azimuth measured in the TPP-wind system and $\nu_{0}, \nu_{0}$, and $\nu_{\text {. are }}$ the wake degrees of freedom (uniform, lateral, and longitudinal). The theory of dynamic inflow relates these states to the aerodynamic loads on a rotor (thrust, pitching moment, and rolling moment). There are two drawbacks to the way the theory is presented in the original paper by Pitt and Peters ${ }^{[30]}$ and most work that follows. First, the model is a perturbation model suited for linear analysis. Second, the formulation is presented in a wind coordinate system making it less suitable for a full computer simulation. These problems are addressed in a paper by Peters and HaQuang ${ }^{[14]}$ where a more suitable rotor frame system is used and a nonlinear version is also included. The remainder of this section will present the inflow equations of reference [14] and define its components in terms of the notation used in this thesis.

The dynamic inflow model is given by the following differential equations:

$$
[., U]\left\{\begin{array}{l}
\bar{\lambda}_{0} \\
\bar{\lambda}_{s} \\
\bar{\lambda}_{c}
\end{array}\right\}+[\hat{L}]^{-1}\left\{\begin{array}{l}
\lambda_{0} \\
\lambda_{s} \\
\lambda_{c}
\end{array}\right\}=\left\{\begin{array}{r}
C_{T} \\
C_{1} \\
-C_{2}
\end{array}\right\}_{\text {zero }}
$$

where $\left(\lambda_{0}, \lambda_{3}, \lambda_{c}\right)$ define the inflow states as do $\left(\nu_{0}, \nu_{s}, \nu_{c}\right)$ but are in a TPP.Body axes system. The matrix $[M]$ is an apparent mass matrix and $[\hat{L}]$ is a nonlinear version of the 
inflow gain matrix transformed to the TPP.body system. The coefficients $C_{T}, C_{1}$, and $C_{2}$ are the thrust. roling, and pitching moment coefficients respectively: the subscript "aero" Innotes that these are due to aerodynamic loads only. The teetering rotor of the Bell 205 does not produce a net moment. however. considerable aerodynamic moments may exist which are cancelled out by the inertial moments. The inflow variation over the rotor disk in the TPP body system is:

$$
\lambda\left(r, L_{w}\right)=\lambda_{0}+\lambda_{s} x \sin \psi_{k}+\lambda_{c} x \cos \dot{\psi}_{k}
$$

and the matrix $[. M]$ and $[\hat{L}]^{-1}$ are defined:

$$
\begin{gathered}
{[. M]=\left[\begin{array}{ccc}
\frac{128}{i 5 \pi} & 0 & 0 \\
U & \frac{16}{15 \pi} & 0 \\
0 & 0 & \frac{16}{45 \pi}
\end{array}\right]} \\
{[\dot{L}]^{-1}=[V][\xi]^{-1}}
\end{gathered}
$$

where.

$$
\begin{gathered}
{[V]=\text { mass flow parameter matrix }} \\
=\left[\begin{array}{ccc}
V_{T} & 0 & 0 \\
0 & V & 0 \\
0 & 0 & V
\end{array}\right] \\
{[\xi]=\left[\begin{array}{ccc}
1 / 2 & -B \cos \Delta \\
B \sin \Delta & E \cos ^{2} \Delta+D \sin ^{2} \Delta \quad(D-E) \sin \Delta \cos \Delta \\
B \cos \Delta & (D-E) \cos \Delta \sin \Delta \quad E \sin ^{2} \Delta+D \cos ^{2} \Delta
\end{array}\right]} \\
E=\frac{4}{1+\sin x}, \quad D=\frac{4 \sin x}{1+\sin x} \\
\quad B=\frac{15 \pi}{64} \sqrt{\frac{1-\sin x}{1+\sin x}} \\
x=\frac{\tan ^{-1}\left[\frac{\left|\lambda_{m}-\mu_{3}\right|}{\mu}\right]}{V}=\frac{\mu^{2}+\left(2 \lambda_{m}-\mu_{3}\right)\left(\lambda_{m}-\mu_{3}\right)}{V_{T}} \\
\lambda_{m}=\frac{1}{2}\left\{\begin{array}{l}
1 \\
0 \\
0
\end{array}\right\} \begin{array}{l}
{[\xi]^{-1}\left\{\begin{array}{l}
\lambda_{0} \\
\lambda_{s} \\
\lambda_{c}
\end{array}\right\}}
\end{array}
\end{gathered}
$$


Here, $V_{T}$ is the total resultant flow through the rotor disk. $I$ is the inflow mass-flow parameter, and $x$ is the wake angle measured from rotor disk. The remaining terms are most easily described using figure $\mathbf{2 . 9}$ modified from reference [1 14 . The coordinate system $\left(\bar{b}_{1}^{T}, \bar{b}_{2}^{T}, \dot{b}_{3}^{T}\right)$ is a TPP-body coordinate system with $\left(\dot{b}_{1}^{T}, \dot{b}_{2}^{T}\right)$ defining the tip-path-plane and $\hat{b}_{3}^{T}$ completing the right handed coordinate system. These axes are a body systent because $\hat{b}_{1}^{T}$ remains in the longitudinal plane of the helicopter and $\hat{b}_{2}^{T}$ remains in the lateral plane. Thus the azimuth measured from $\dot{b}_{i}^{T}$ is the same as that measured from $\dot{r}_{\dot{s}}$ and therefore corresponds to notation used thus far. It should be clarified that $\dot{b}_{i}^{r}$ and $\boldsymbol{b}_{2}^{r}$ can only be expected to remain in the longitudinal and lateral planes when one or both of the flapping angles $\theta_{R}$ and $\phi_{k}$ are zero. When both are non-zero these axes deviate from their desired planes, but do so in a negligible fashion since the flapping angles are small. The axes $\left(\hat{b}_{1}^{\omega}, \hat{b}_{2}^{\omega}, \hat{b}_{3}^{\omega}\right)$ are the TPP-wind coordinate system that are used for most rotor-only analysis including dynamic inflow (Pitt's original model is in this system).

The resultant normalized velocity of oncoming flow is $\vec{V}_{R}$, which breaks down into $\mu$ and $\mu_{3}$ in the TPP-wind system. The components of $\mu$ in the TPP-body system are $\mu_{1}$ (the longitudinal velocity) and $\mu_{2}$ (the lateral velocity). The resultant flow through the rotor disk is:

$$
V_{T}=\sqrt{\left(\lambda_{m}-\mu_{3}\right)^{2}+\mu^{2}}
$$

where $\lambda_{m}$ is the non-dimensional induced velocity due to rotor thrust derived using momentum theory. The quantity $\left(\lambda_{m}-\mu_{3}\right)$, which is the total inflow through the rotor, is given the symbol $\lambda$.

$$
\lambda=\lambda_{m}-\mu_{3}
$$

The angle, $\Delta$, between the TPP-wind and TPP-body systems is given by:

$$
\begin{aligned}
& \sin \Delta=\frac{\mu_{2}}{\mu}=\frac{\mu_{2}}{\sqrt{\mu_{1}^{2}+\mu_{2}^{2}}} \\
& \cos \Delta=\frac{\mu_{1}}{\mu}=\frac{\mu_{1}}{\sqrt{\mu_{1}^{2}+\mu_{2}^{2}}}
\end{aligned}
$$

In a computer simulation the dynamic inflow equations just described can be solved in the following manner:

1. Calculate the TPP-body velocities $\left(\mu_{1}, \mu_{2}, \mu_{3}\right)$ and the aerodynamic coefficients $\left(C_{T}, C_{1}, C_{2}\right)$. 

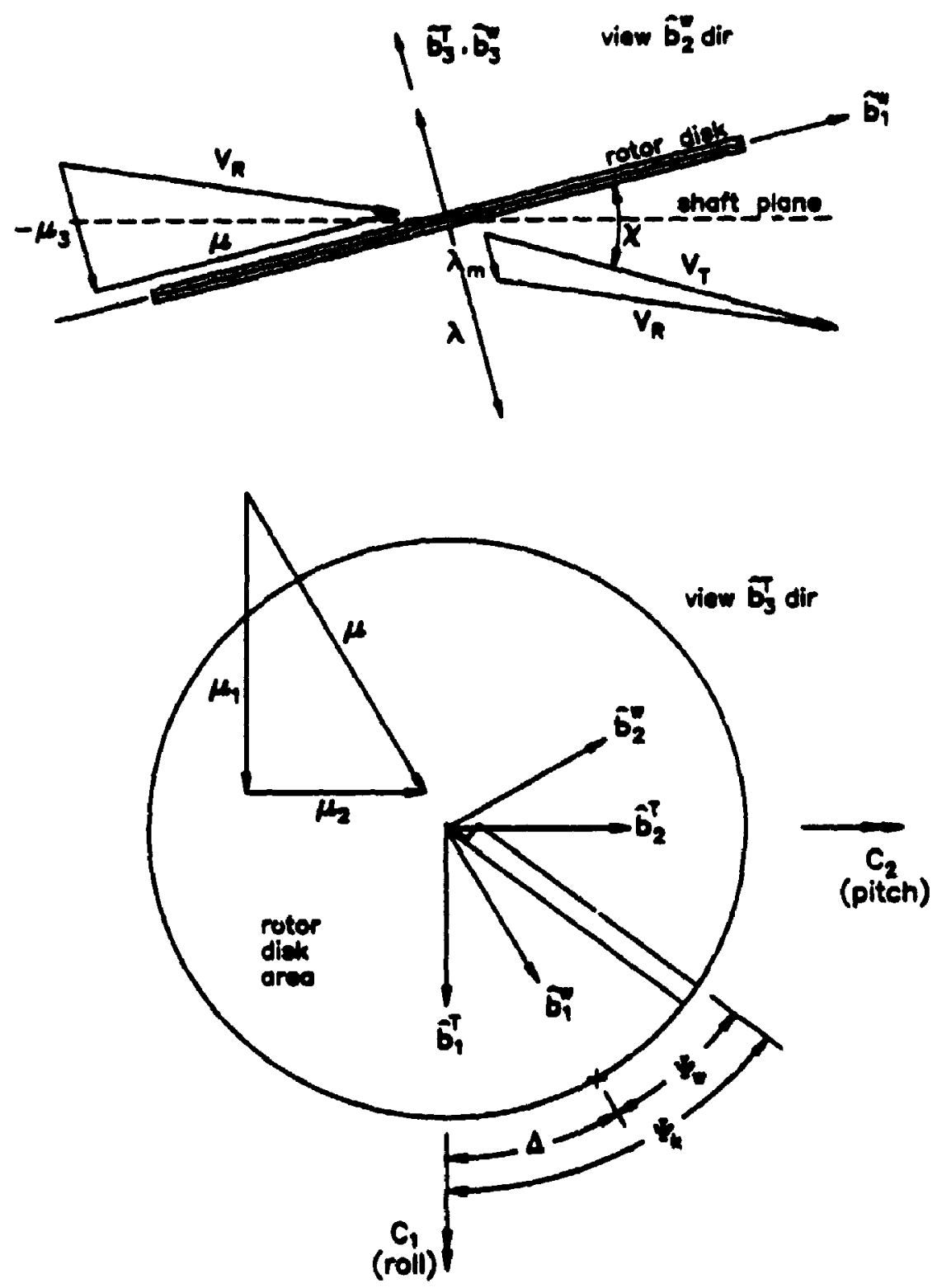

Figure 2.9: Airflow geometry at rotor disk 


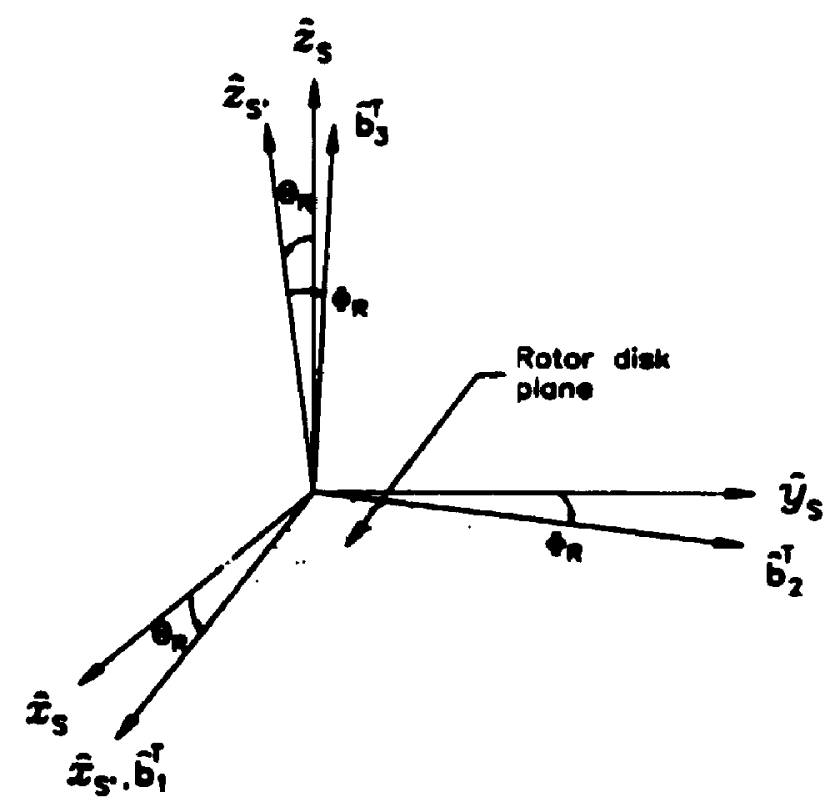

Figure 2.10: Translation of shaft axes to TPP axes.

2. Use $\left(\lambda_{0}, \lambda_{s}, \lambda_{c}\right)$ and $\times$ from the last time step to solve equation $(2.30)$ for $\lambda_{r n}$.

3. Find the new value of $x$ by using the above $\lambda_{m}$ in equation (2.28).

4. Solve differential equation (2.23) and use it to propagate the inflow states.

The relations required to perform step 1 above will now be derived. The required transformation from the shaft axis to the TPP-body system is shown in figure 2.10. A rotation of $\theta_{R}$ about $\hat{y}_{s}$ followed by a rotation of $-\phi_{n}$ about $\hat{x}_{s^{\prime}}$ will take vectors from the shaft axis to the TPP-body system (" $A^{n}$ system), labelled with subscripts 1 , 2 , and 3 . It should be clarified that this use of $\phi_{n}$ does not strictly comply with its definition (the angle from $\hat{y}_{s}$ to the TPP). This set of transformations have been used because they force i to be in the longitudinal plane of the helicopter which is important for measurement of azimuth. The y component of the lesulting coordinate system will vary slightly form the lateral plane at times as discussed earlier. The transformation matrix is:

$$
\left[T_{A} S\right]=\left[\begin{array}{ccc}
C \theta_{R} & 0 & -S \theta_{R} \\
-S \phi_{n} S \theta_{R} & C \phi_{R} & -S \phi_{n} C \theta_{R} \\
C \phi_{n} S \theta_{R} & S \phi_{n} & C \phi_{R} C \theta_{R}
\end{array}\right]
$$


Thus can be used to derive the TPP velocities according to:

$$
\begin{aligned}
& \left\{\begin{array}{l}
\mu_{1} \\
\mu_{2} \\
\mu_{3}
\end{array}\right\}=i T_{A S}: \frac{1}{\Omega R}\left(-\bar{s}_{\xi}\right)=\left[T_{A S}\right]\left[T_{S F}\right] \frac{1}{\Omega R}\left(-_{F} \bar{c}_{\xi}\right)=\left[T_{A S}\right]\left\{\begin{array}{l}
\mu_{x} \\
\mu_{y} \\
\mu_{z}
\end{array}\right\} \\
& =\left[\begin{array}{l}
\mu_{x} C \theta_{R}-\mu_{z} S \theta_{R} \\
-\mu_{x} S \phi_{R} S \theta_{R}+\mu_{y} C O_{R}-\mu_{z} S o_{R} C \theta_{F} \\
\mu_{x} C o_{R} S \theta_{R}+\mu_{y} S o_{R}+\mu_{z} C o_{R} C \theta_{R}
\end{array}\right]
\end{aligned}
$$

where ${ }_{s} \vec{v}_{s}$ is the hub velocity and $\left\{\mu_{x} \mu_{y} \mu_{z}\right\}$ are the normalized wind velocity at the hub. all in the shaft coordinate system.

The thrust coefficient, $C_{T}$. is:

$$
C_{T}=\frac{T}{\rho \pi \Omega^{2} R^{4}}
$$

Here, $\mathrm{T}$ is the thrust perpendicular to the tip-path-plane and is given by integrating $d L_{y} \cos c_{o}$ along the blade and averaging over the azimuth. As a result of the sionplifying assumption made for the derivation of thrust along the shaft $\left(d L_{s} \cos 3_{k} \approx d L_{s}\right)$, it is possible to multiply the thrust of equation (2.21) by $\cos c_{0}$ and use it here.

The differential pitching and rolling moments are obtained by taking the differentias force perpendicular to the TPF and multiplying it by the moment arm to the longitudinal and lateral axes $\hat{b}_{1}^{T}$ and $\hat{b}_{2}^{T}$ respectively.

$$
\begin{aligned}
d R M= & d L_{g} \cos c_{0} r \sin \psi_{k} \\
R M= & \frac{k_{c} \rho c \Omega^{2} R^{4}}{2 \pi} \int_{0}^{2 \pi}\left[\int_{x 0}^{B P} a\left(\bar{U}_{T}^{2} \theta-\bar{U}_{T} \bar{U}_{P}\right) x d x\right. \\
& \left.-\int_{0}^{1} c_{d} \bar{U}_{T} \bar{U}_{P} x d x\right] \sin \psi_{k} d \psi_{k} \\
d P M= & d L_{s} \cos c_{0} r \cos \psi_{k} \\
P M= & \frac{k_{c} \rho c \Omega^{2} R^{4}}{2 \pi} \int_{0}^{2 \pi}\left[\int_{x o}^{B P} a\left(\bar{U}_{T}^{2} \theta-\bar{U}_{T} \bar{U}_{P}\right) x d x\right. \\
& \left.-\int_{0}^{1} c_{d} \bar{U}_{T} \bar{U}_{P} x d x\right] \cos \psi_{k} d \psi_{k}
\end{aligned}
$$

The required pitching and rolling moment coefficients are thus:

$$
C_{1}=\frac{R M}{\rho \pi \Omega^{2} R^{5}}, \quad C_{2}=-\frac{P M}{\rho \pi \Omega^{2} R^{5}}
$$




\subsection{Tail Rotor}

The tail rotor of the Bell 205 is a teetering rotor with zero precone angle and zero rotor undersling. The rotor is also without cycisc pitch and blade twist. Due to the high frequency of flapping brought on by the higher rotational rate. the flapping dynamics of the tail rotor can be neglected. The procedure outlined in section 2.3 is used here with minor modification to derive the tail rotor equations.

A set of coordinate systems similar to those of figure 2.3 is adopted to model the tail rotor. The hub $(\mathrm{H})$, modified hub $\left(\mathrm{H}^{\prime}\right)$, and the supplementary blade axes are now identical. and the origin of all axes are at the blade flapping hinge.

\subsubsection{Flapping Angle}

For the tail rotor, the rotor flapping approximation of equation (2.2) reduces to:

$$
\begin{aligned}
& 3_{T R}(t)=\theta_{R_{T R}} \cos (\psi)-\phi_{R_{T R}} \sin (\psi) \\
& \dot{j}_{T R}(t)=-\phi_{R_{T R}} \Omega_{T R} \cos (\psi)+\theta_{R_{T R}} \Omega_{T R} \sin (\psi) \\
& \ddot{J}_{T R}(t)=\theta_{R_{T R}} \Omega_{T R}^{2} \cos (\psi)+\phi_{R_{T R}} \Omega_{T R}^{2} \sin (\psi)
\end{aligned}
$$

The absolute angular velocity of the rotor can be expressed as:

$$
\left({ }_{H^{\prime}} \overrightarrow{\bar{\omega}}_{H^{\prime} / 1}\right)_{T R}=T_{H R} \cdot T_{R S} \cdot T_{S_{T R} F} \cdot \overrightarrow{\bar{\omega}}_{F / I}+T_{H R} \cdot \Omega_{T R} \dot{z}_{R}-\dot{j}_{b_{T R}} \dot{y}_{H}
$$

and the velocity of a blade element $P$ becomes:

$$
\begin{aligned}
{ }_{B^{\prime}} \vec{v}_{P}= & { }_{B^{\prime}} \vec{v}_{S}+{ }_{B^{\prime}} \vec{v}_{P / S}+\text { main rotor effect } \\
= & T_{B^{\prime} R} \cdot T_{R S} \cdot T_{S_{T R} F}\left(\vec{v}_{F}+\vec{w}_{F / I} \times \vec{r}_{S_{T R} / F}\right)+\left(T_{B^{\prime} R} \cdot T_{R S} \cdot T_{S_{T R} F F} \vec{w}_{F / I}\right. \\
& \left.+T_{B^{\prime} R} \cdot\left\{0-\dot{3}_{k_{T R}} \Omega_{T R}\right\}^{T}\right) \times r \hat{x}_{B^{\prime}}+w_{T R} \dot{y}_{S_{T R}}
\end{aligned}
$$

where,

$$
\begin{aligned}
\vec{r}_{S_{T R} / F} & =-X_{S_{T R} F} \hat{x}_{F}+Y_{S_{T R} F} \hat{y}_{F}-Z_{s_{T R} F} \hat{z}_{F} \\
w_{i R} & =\text { main rotor interference term } \\
T_{S_{C R} F} & =\left[\begin{array}{rrr}
-1 & 0 & 0 \\
0 & 0 & 1 \\
0 & 1 & 0
\end{array}\right]
\end{aligned}
$$


The above can be solved to yield the blade element velocities:

$$
\begin{aligned}
& r_{T_{T R}}=\left(\dot{\theta}+\Omega_{T R}\right) r+\cos v_{k}\left(u_{F}+u_{T R}+\dot{\theta} X_{s_{T R} F}+\dot{o} Y_{s_{T R} F}+\dot{o r} 3_{K_{T R}}\right) \\
& +\sin v_{k}\left(u_{F}-i \gamma_{s_{T R} F}-\dot{o} Z_{s_{T R} F}-\dot{i} \cdot 3_{k_{T R}}\right) \\
& l_{P_{T H}}=\dot{j}_{K_{T R}} r+v_{F}-u x_{s_{T R} F}+\dot{O} Z_{s_{T A} F} \\
& +\cos u_{k}\left(-\dot{u} r+u_{F} 3_{k_{T R}}-\dot{v} Y_{s_{T R} F} 3_{k_{T R}}-\dot{\theta} Z_{s_{T R} F} 3_{k_{T R}}\right) \\
& +\sin w_{k}\left(-\dot{o} r-w_{F} 3_{k_{T R}}-w_{T R} \beta_{k_{T R}}-\dot{\theta} X_{s_{T R} F} \beta_{k_{T R}}-\dot{o} Y_{s_{T R} F} 3_{k_{T R}}+v_{T T R}\right.
\end{aligned}
$$

where $v_{\text {TR }}$ is the mean tail rotor induced velocity. A momentum theory expression for induced velocity that is applicable for both forward velocity and hover can be found in reference [29]:

$$
v_{T R}=\frac{T}{2 \rho \pi R_{T R}^{2} B^{2} V^{\prime}}
$$

where

$$
\begin{aligned}
T & =\text { tail rotor thrust } \\
\rho & =\text { air density } \\
R_{T R} & =\text { tail rotor radius } \\
B & =\text { tip loss factor from }(2.16) \approx 0.97 \\
V^{\prime} & =\sqrt{\left(v_{T R}-V_{T R} \sin \alpha_{R}\right)^{2}+\left(V_{T R} \cos \alpha_{R}\right)^{2}} \\
& =\text { total velocity through the rotor } \\
V_{T R} & =\text { velocity of on coming flow } \\
\alpha_{R} & =\text { rotor angle of attack } \\
& =\text { taken as angle between shaft plane and } V_{T R}
\end{aligned}
$$

To account for transient flow changes resulting from thrust variations, an "apparent additional mass" of air term, $m_{a}$, is introduced to account for the flow acceleration through the tail rotor ${ }^{[31]}$ :

$$
T=m_{a} \dot{v}_{T R}+2 \rho \pi R_{T R}^{2} B^{2} V^{\prime} v_{t R}
$$

where $m_{a}$ is defined to be $63.7 \%$ of the air mass of the circumscribed sphere defined by the tail rotor radius.

The remaining equation derivations are identical to that performed for the main rotor and will be omitted. 
The tail rotor happing angles are found by simultaneously solving equations $(2.16)$ and (2.19) for the tail rotor:

$$
\begin{aligned}
& \theta_{R_{T R}}=\frac{F 3(F 1 F 2-2 F+F 5)}{6 F 1(-F 3 F 4+12 F 1 F 6)}-\frac{F 5}{3 F 1}+\text { h.o.t } \\
& o_{R_{T A}}=-\frac{F 1 F 2-2 F 4 F 5}{12 F 1 F 6-F 3 F 4}+\text { h.o.t }
\end{aligned}
$$

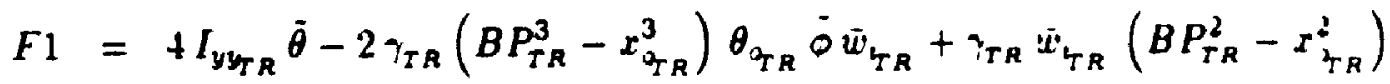

$$
\begin{aligned}
& \left(\bar{u}_{F}-\tilde{\theta} \bar{Z}_{s_{T R} F}\right) \\
& F 2=12\left(3 \gamma_{T R}\left(B P_{T R}^{4}-x_{\mathrm{o}_{T R}}^{4}\right) \dot{\phi}+6 I_{y y_{T R}}(\dot{\bar{\phi}}-2 \dot{\psi})\right. \\
& +8 \gamma_{T R} \theta_{\partial_{T R}}\left(B P_{T R}^{3}-x_{\partial_{T R}}^{3}\right)\left(\bar{u}_{T}-\bar{\theta} \bar{L}_{S_{T R^{F}}}\right) \\
& \left.+6 \gamma_{T R} \bar{u}_{T R R}\left(B P_{T R}^{2}-x_{T_{T R}}^{2}\right)\left(\tilde{\theta} \bar{Z}_{s_{T R} F}-\bar{u}_{F}\right)\right) \\
& F 3=\gamma_{T R}\left(6\left(B P_{T R}^{4}-x_{q_{T R}}^{4}\right)(1+\bar{\theta})+4\left(B P_{T R}^{3}-x_{q_{T R}}^{3}\right) \theta_{T_{T R}} \bar{\psi}_{\bar{\psi}} \bar{\psi}_{T R}\right. \\
& \left.+6\left(B P_{T R}^{2}-x_{Q_{T R}^{2}}^{2}\right) \bar{w}_{i_{T R}}\left(\bar{\theta} \bar{X}_{s_{T R F}}-\bar{w}_{F}\right)\right) \\
& F 4=\gamma_{T R}\left(-6\left(B P_{T R}^{4}-x_{\sigma_{T R}}^{4}\right)(1+\bar{\theta})+4\left(B P_{T R}^{3}-x_{\partial_{T R}}^{3}\right) \theta_{T_{T R}} \bar{\psi} \bar{\omega}_{T R}\right. \\
& \left.+6\left(B P_{T R}^{2}-r_{q_{T R}^{2}}^{2}\right) \bar{w}_{L_{T R}}\left(\tilde{\theta} \bar{X}_{S_{T R} F}-\bar{w}_{F}\right)\right) \\
& F 5=6 I_{y y_{T R}}(\tilde{\dot{\psi}}+\bar{\phi})+\gamma_{T R}\left\{3\left(B P_{T R}^{4}-x_{o_{T R}}^{4}\right) \tilde{\psi}\right. \\
& +8\left(B P_{\tau R}^{3}-x_{T_{T R}}^{3}\right) \theta_{O_{T R}}\left(\bar{w}_{F}+\bar{w}_{i_{T R}}+\bar{w}_{i_{T R}} \bar{\theta}\right) \\
& +6\left(B P_{T R}^{2}-x_{q_{T R}}^{2}\right)\left(\bar{v}_{T R}\left(-\bar{w}_{F}-\dot{\theta} \bar{X}_{s_{T R} F}-\bar{w}_{T R}\right)\right. \\
& \left.\left.+6 \bar{w}_{i_{T R}}\left(-\bar{v}_{F}+\bar{\psi} \bar{X}_{s_{T R F}}-\dot{\phi} \bar{\nu}_{s_{T R F}}\right)\right)\right\} \\
& F 6=12 I_{y y_{T R}} \bar{\theta}+\gamma_{T R} \bar{w}_{T R}\left(-2\left(B P_{T R}^{3}-x_{T_{T R}}^{3}\right) \theta_{T_{T R}} \bar{\phi}\right. \\
& \left.+3\left(B P_{T R}^{2}-x_{q_{T R}}^{2}\right)\left(\bar{\theta} \bar{z}_{s_{T R^{F}}}-\bar{u}_{r}\right)\right)
\end{aligned}
$$




$$
\gamma_{T R}=\frac{1}{2} \rho_{T R} a_{T R} c_{T R} R_{T R}^{4}
$$

where $a_{T R}, c_{T R}$, and $\rho_{T R}$ are the tail rotor lift curve slope, blade cord. and air density respectively. The tail rotor quantities are nondimensionalized with the tail rotor radius. $R_{T R}$ and rotational rate. $\Omega_{T R}$.

\subsubsection{Tail Rotor Aerodynamic Loads}

The rotor loads are calculated according to the formulation outlined in section 2.3.4. The only difference being the thrust reduction factor which is used to account for the interaction between the tail rotor and the vertical tail.

A study carried out by the Bell Helicopter company[21] showed that the impingement of tail rotor wake on the vertical tail caused significant thrust reduction. The net thrust for a tractor tail configuration was found to vary approximately as:

$$
T_{\text {net }}=T(1-0.75 \stackrel{S}{A})
$$

where $A$ is the tail rotor disk area and $S$ is the disk area blocked by the vertical fin: The ratio $\frac{S}{A}$ is approximately 0.14 for the Bell 205 resulting in a $10 \%$ thrust reduction.

\subsubsection{Main Rotor Interference}

The main rotor downwash/upwash at the tail rotor is approximated by way of induced velocity charts found in references [32] and [33]. These charts give the time-averaged induced velocities near a lifting rotor at various wake angles. The model used to incorporate these charts into the computer simulation is taken from reference [22] and is presented in this subsection.

Figure 2.11 shows an induced velocity chart as presented in reference [33]. It is a contour plot of induced velocity ratio $\left(\frac{w_{x}}{v_{1}}\right)$ in the longitudinal plane of the rotor at zero wake angle and triangular disk loading. Triangular disk loading has been chosen over uniform disk loading because it better represents the actual rotor and it is consistent with the earlier derivations. Charts are given for eight wake angles in the longitudinal plane and five wake angles in the lateral plane. The coordinate system used in reference [33] is the TPP-wind system used for dynamic inflow but labelled $(X, Y, Z)$ instead of $\left(\hat{b}_{1}^{T}, \hat{b}_{2}^{T}, \hat{b}_{3}^{T}\right)$. The location of a point on the body in this coordinate system is a function of TPP tilt. 


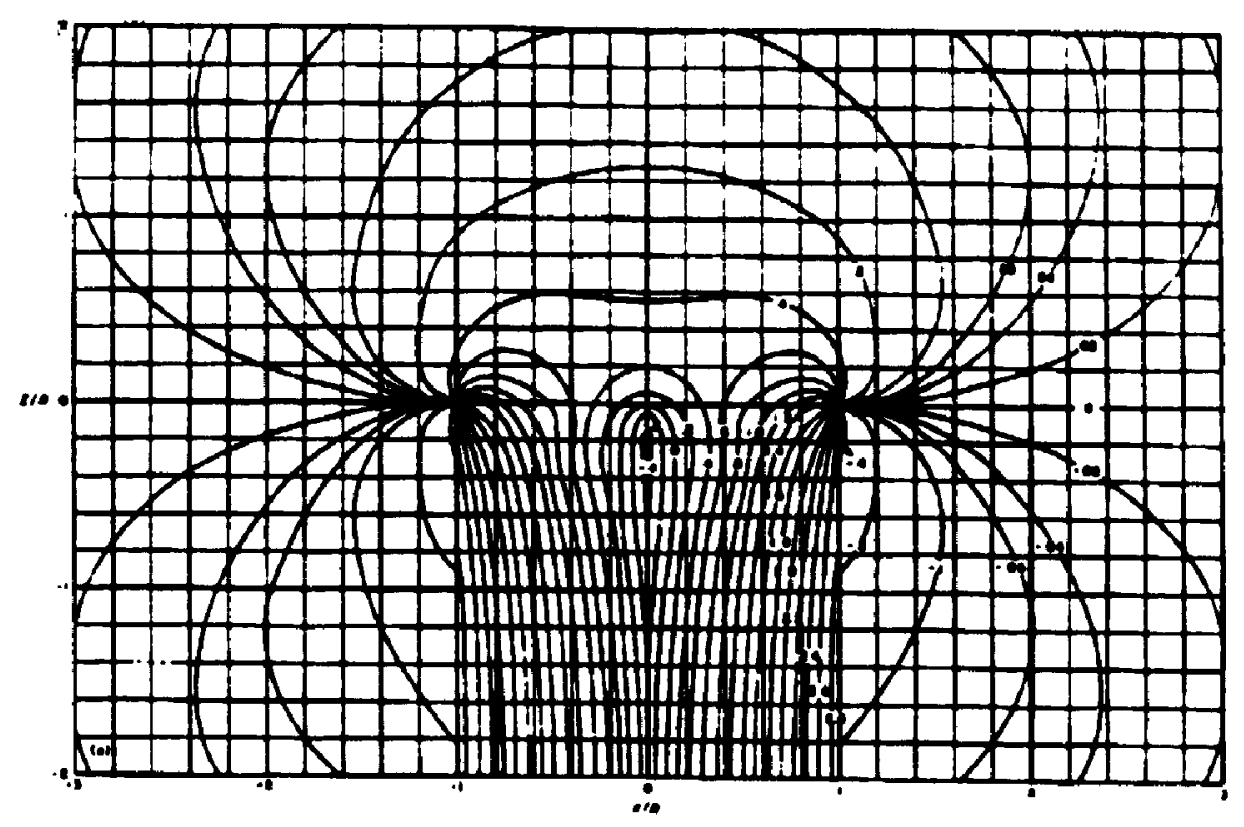

Figure 2.11: Longitudinal induced velocity ratio $(x=0)^{[33]}$

Due to the approximate nature of the wake estimate, which can vary widely with disk load distribution, inclusion of this level of detail may be unwarranted. A simpler wake model may be obtained if a hub-wind coordinate system is employed with the wake angle and tcil position referred to that system. Therefore:

$$
x=\tan ^{-1}\left(\frac{\mu}{\lambda}\right)
$$

where

$$
\begin{aligned}
& \mu=\sqrt{\mu_{x}^{2}+\mu_{y}^{2}} \\
& \lambda=\lambda_{m}-\mu_{z}
\end{aligned}
$$

The downwash can now be expressed as a power series in wake angle at angles of sideslip of $0^{\circ}, \pm 90^{\circ}$, and $180^{\circ}$ according to:

$$
\left(\frac{w_{i n R}}{v_{i}}\right)=\sum_{n=0}^{4} k_{n} x^{n}
$$

where the factors $k_{n}$ are determined by a least-squares fit to the data obtained from the velocity charts and $v_{i}$ is the momentum theory induced velocity produced by the main rotor $\left(v_{i}=\lambda_{m} \Omega R\right)$. Wake angles between $0^{\circ}$ and $84^{\circ}$ are only considered since the main 
foror model is only valid to tip speed ratios of $\mu=0.3$ (given that $\lambda$ is approximately $0.00 \times 1$ at these speeds). The resulting downwash ratio expressions for $X / R=1.2$ and $Z_{i} R=-0.1$ are.

$$
\frac{u_{T R}}{v_{t}}= \begin{cases}0.15 i-0.768 x+0.209 \chi^{2}-2.007 x^{3}+1.186 \chi^{4} & 3_{u}=0^{\circ} \\ 0.150+0.219 x-0.063 \chi^{2}+0.581 \chi^{3}-0.302 \chi^{4} & 3_{w}= \pm 90^{\circ} \\ 0.146+0.153 x+0.575 \chi^{2}-0.727 \chi^{3}+0.232 \chi^{4} & 3_{w}=180^{\circ}\end{cases}
$$

where the sign of each expression has been chosen to correspond to the earlier definition of $w_{\text {TR }}$ (i.e. positive for upwash). The relative fit of these functions to the induced velocity data are shown in figure 2.12. Downwash at arbitrary sideslip angles can be interpolated according to:

$$
\frac{w_{T R}}{v_{z}}= \begin{cases}{\left[\left(\frac{w_{T R}}{v_{1}}\right)_{3_{w}=00}\left|\cos 3_{w}\right|+\left(\frac{w_{T R}}{v_{1}}\right)_{s_{\nu=90}}\left|\sin 3_{w}\right|\right]} & \left|3_{w}\right| \leq 90^{\circ} \\ {\left[\left(\frac{w_{T R}}{v_{1}}\right)_{s_{w}=180^{\circ}}\left|\cos 3_{w}\right|+\left(\frac{w_{T R}}{v_{1}}\right)_{s_{w}=90^{\circ}}\left|\sin 3_{w}\right|\right]} & 90^{\circ}<\left|3_{w}\right|<270^{\circ}\end{cases}
$$

where $3_{w}$ is the complex sideslip angle defined by:

$$
3_{w}=-\tan ^{-1}\left(\frac{\mu_{y}}{\mu_{x}}\right)
$$

and $\lambda_{w}=0$ for $\mu_{x}=\mu_{y}=0$.

\section{Tail Rotor Contribution to the Total Forces and Moments}

The orientation of the forces and moments produced by the tail rotor are shown in fig. ure 2.1. It follows that:

$$
\begin{aligned}
& F_{I_{T R}}=-H_{T R} \\
& F_{Y_{T R}}=T_{\text {net }} \\
& F_{\text {YXR }}=Y_{\text {TR }} \\
& M_{x_{T R}}=T_{\text {netR }} Z_{s_{T R} F} \\
& M_{Y_{T R}}=H_{T R} z_{s_{T R F}}+Y_{T R} X_{s_{T R F}}-Q_{T R} \\
& M_{\tau_{T R}}=-T_{\text {net }} X_{s_{T R} F}+H_{T R} X_{s_{T R} F}
\end{aligned}
$$

where $F_{T_{T R}}, F_{Y_{T R}}$ and $F_{Y R}$ are the longitudinal, lateral, and vertical forces respectively in the fuselage coordinate system and $M_{x_{T R}}, M_{Y_{T R}}$, and $M_{Y_{T R}}$ are the rolling, pitching, and yawing moments respectively. 

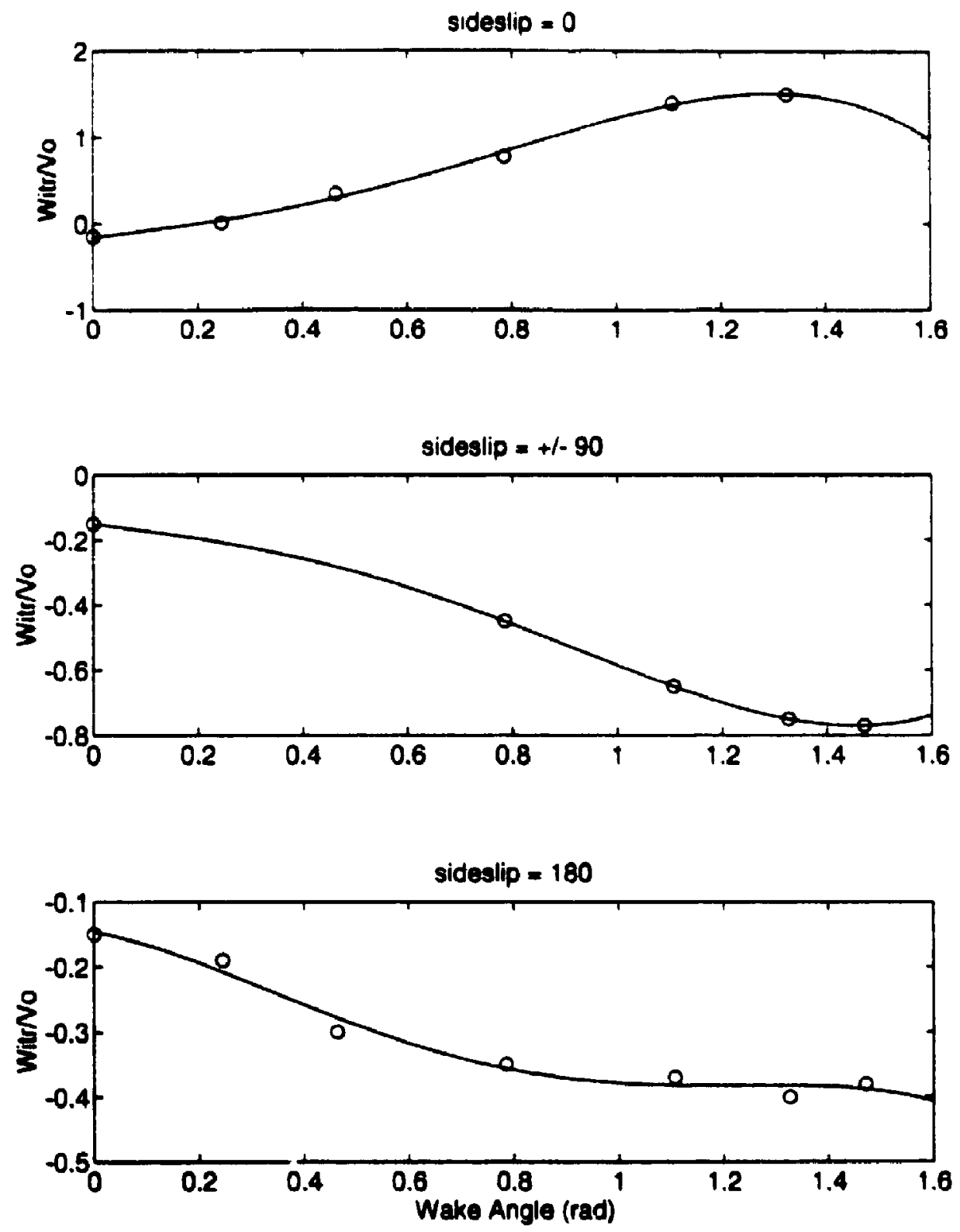

Figure 2.12: Least-squares fit of downwash at tail rotor 


\subsection{Empennage}

The horizontal stabilizer and vertical fin models apply to the full operating range of the helicopter including sideward, vertical, and rearward fight. Main rotor interference effects are accounted for on both surfaces and tail rotor interference effects are accounted for on the vertical fin.

The model used for the empennage surfaces is that of reference [22] modified to account for the variation of main-rotor downwash across the span of the horizontal stabilizer. Reference [22] makes the following assumptions in developing the expressions for lift and drag on the empennage surfaces:

1. The lift and drag forces are applied at the quarter chord of each surface at the spanwise location of the centre of area.

2. The airfoil profiles are symmetrical.

3. The lift curve slope prior to stall is given by simple lifting-line theory assuming an elliptical lift distribution with uniform downwash. Corrections are applied for sideslip and for sweep of the vertical fin.

4. Post stall variation of lift coefficient is based on $C_{L_{m}}$ decreasing by $20 \%$ as the angle of attack is increased by $20 \%$, and following a particular variation thereafter to reach zero lift at an angle of attack of $\pi / 2$.

5. Lift coefficient in rearward flight is $80 \%$ of that in forward flight.

6. The profile drag coefficient varies as the square of the calculated lift coefficient.

For this study, assumption 1 is not used since the centre of pressure data are available and thus the lift and drag forces are applied at the centre of pressure. Assumption 2 is only used to simplify the variation of lift and drag for the full range of angle of attack, however. in the calculation of angle of attack the effects of airfoil camber are accounted for.

The variation of the lift and drag coefficients for the full range of angle of attack can be found in reference [34] where the typical variation is as shown in figure 2.13. This figure 

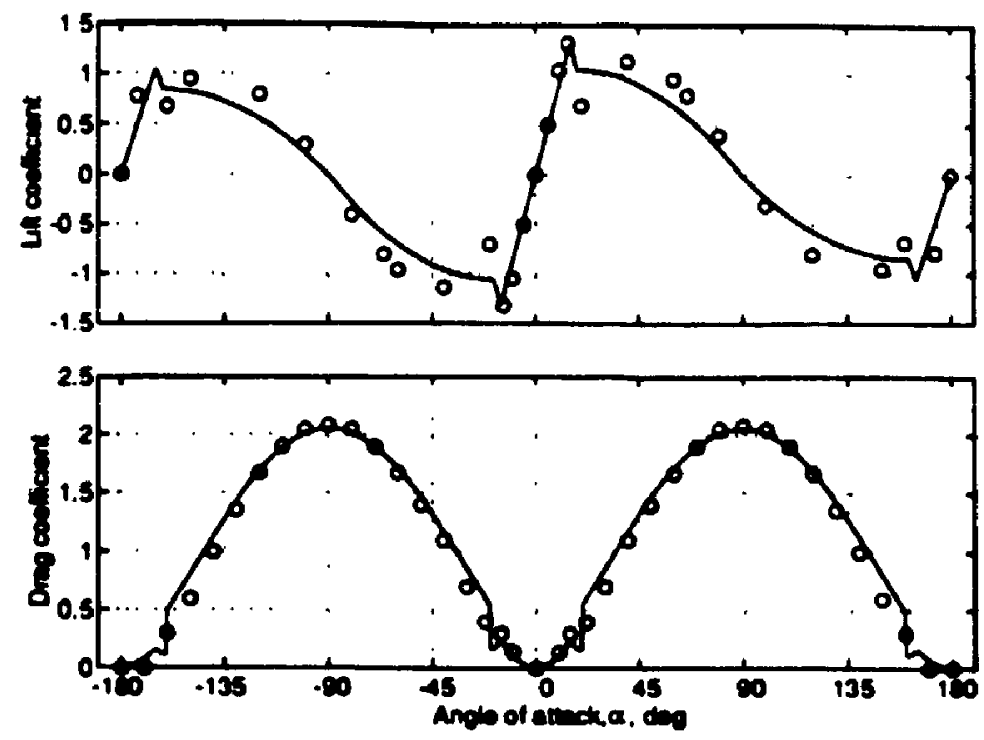

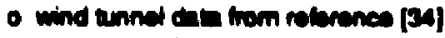

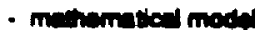

Figure 2.13: Typical variation of lift and drag on empennage surfaces

is applicable to both empennage surfaces and can be expressed mathematically as:

$$
\begin{aligned}
& C_{L_{0}}=\left\{\begin{array}{lc}
a \alpha_{i} & 0 \leq \alpha_{i}<\alpha_{,} \\
C_{L_{m}}-a\left(\alpha_{i}-\alpha_{s}\right) & \alpha_{s}<\alpha_{i}<\alpha_{1} \\
0.8 C_{L_{m}}\left[1-\left(\frac{a-\alpha_{2}}{\pi / 2-\alpha_{1}}\right)^{2}\right] & \alpha_{1} \leq \alpha_{i} \leq \frac{\pi}{2}
\end{array}\right. \\
& C_{D_{p}}=\left\{\begin{array}{lc}
0.009+0.11 \alpha_{i}^{2} & 0 \leq \alpha_{i} \leq 0.35 \\
-0.1254+0.9415 \sin \alpha_{i}+0.977525 \sin ^{2} \alpha_{i} & 0.35<\alpha_{i}<\frac{\pi}{2}
\end{array}\right. \\
& C_{D}=C_{D_{p}+\frac{C_{L}^{2}}{0.8 \pi A . R}} \\
& C_{L}=\left\{\begin{array}{lc}
C_{L_{0}} & 0 \leq \alpha<\frac{\pi}{2} \\
-C_{L_{0}} & -\frac{\pi}{2} \leq \alpha<0 \\
-0.8 C_{L_{0}} & \frac{\pi}{2} \leq \alpha<\pi \\
0.8 C_{L_{0}} & -\pi \leq \alpha<-\frac{\pi}{2}
\end{array}\right.
\end{aligned}
$$

In the above expressions $C_{L}$ is the surface lift coefficient, $C_{D}$ is the drag coefficient, $C_{D_{p}}$ is the profile drag coefficient, $a_{\mathrm{a}}$ is the stall angle of attack, $C_{L_{m}}$ is the maximum lift coefficient, $\alpha_{1}$ defines the angle of attack of the upper stall region, and $\alpha_{i}$ is the surface 
angle of attack. $\alpha$. modified to fall between 0 and $\frac{\overline{2}}{2}$ according to:

$$
\alpha_{i}=\left\{\begin{array}{cc}
\alpha & 0 \leq \alpha<\frac{\pi}{2} \\
-\alpha & -\frac{\pi}{2} \leq \alpha<0 \\
\pi-\alpha & \frac{\pi}{2} \leq \alpha<\pi \\
\pi+\alpha & -\pi \leq \alpha<-\frac{\pi}{2}
\end{array}\right.
$$

also

$$
\begin{aligned}
& \alpha_{s}=\frac{C_{L_{m}}}{a} \\
& \alpha_{1}=1.2 \alpha_{s}
\end{aligned}
$$

where $a$ is the lift curve slope.

\subsubsection{Horizontal Stabilizer}

The horizontal stabilizer is modeled as two separate wings with fuselage blockage effects. In the following formulation subscript 1 denotes the right stabilizer and subscript 2 the left.

\section{Velocity}

The velocity experienced by the center of pressure of the stabilizers are:

$$
\vec{v}_{H S_{1,2}}=\vec{v}_{F}+\vec{w}_{F / I} \times \vec{r}_{H S / F_{1,2}}=\left\{u_{H S}, v_{H S}, w_{H S}\right\}_{1,2}
$$

where

$$
\begin{aligned}
& \vec{r}_{H S / F 1}=-X_{F H S} \hat{x}_{F}+Y_{F H S} \hat{y}_{F}-Z_{F H S} \hat{z}_{F} \\
& \vec{r}_{H S / F 2}=-X_{F H S} \hat{x}_{F}-Y_{F H S} \hat{y}_{F}-Z_{F H S} \hat{z}_{F}
\end{aligned}
$$

thus,

$$
\begin{aligned}
u_{H S_{1,2}} & =u_{F}-\dot{\theta} Z_{F H S}-\dot{\psi}\left( \pm Y_{F H S}\right) \\
v_{H S_{1,2}} & =v_{F}+\dot{\phi} Z_{F H S}-\dot{\psi} X_{F H S} \\
w_{H S_{1}} & =w_{F}+\dot{\theta} X_{F H S}-w_{H S .1}+\dot{\phi} Y_{F H S} \\
w_{H S_{2}} & =w_{F}+\dot{\theta} X_{F H S}-w_{H S .2}-\dot{\phi} Y_{F H S}
\end{aligned}
$$


where $w_{i H s_{1,2}}$ are the main rotor downwash at the left and right horizontal stabilizers. defined positive downward. The expressions for $w_{i H s}$ are der'ved in the same manner as for the tail rotor using $X / R=0.77, Z / R=-0.32$ :

$$
\frac{w_{i H S}}{v_{1}}= \begin{cases}1.799-1.456 x+4.489 \chi^{2}-8.410 \chi^{3}+4.023 \chi^{4} & \beta_{w}=0^{\circ} \\ 1.800+2.714 x-1.599 \chi^{2}-2.336 \chi^{3}+1.015 \chi^{4} & \beta_{w}= \pm 90^{\circ} \\ 1.800-1.878 x+9.311 \chi^{2}-3.761 x^{3}-12.026 \chi^{4} & \beta_{w}=180^{\circ}\end{cases}
$$

The above expressions for main-rotor downwash assumes a uniform downwash across the span of the stabilizer. Reference [19] shows that the main-rotor downwash variation can cause an angle of attack variation across the span of the stabilizer of the order of 10 degrees. In addition, downwash variation across the span of the horizontal stabilizer contributes significantly to yaw pitch coupling. These effects are small at low forward speeds and are thus neglected by using the model above up to a tip speed ratio of $\mu=0.064$ or 30 knots. At tip speed ratios greater than $0.00 \hat{4}$ the spanwise variation of main-rotor downwash is calculated in the TPP-wind coordinate system $(x, y, z)$ according to:

$$
v_{z}(y)= \begin{cases}-\frac{10 \Omega C_{x}}{\mu R^{2}}(\mu R+y)\left(2 R-\frac{3 \pi y}{4}\right) y & (R>y>0) \\ -\frac{10 \Omega C_{x}}{\mu R^{2}}(\mu R+y)\left(2 R+\frac{3 \pi y}{4}\right) y & (0>y>-R)\end{cases}
$$

This expression for main rotor downwash is from reference [19] where the flat vortex wake model is used in its derivation. The details of the derivation will not be given here but it should be noted that the velocity distribution at the horizontal tail is assumed to be that on the wake, i.e. at $z=0$. In addition, the distribution is a function of $y$ which is measured from the centerline of the wake, not the fuselage. Therefore in order to account for sideslip the following expression must be used:

$$
y=u_{h}+X_{s H s} \sin \beta_{f}
$$

where $z_{h}$ is measured from the centerline of the fuselage $\left(\hat{z}_{F}-\hat{z}_{F}\right.$ plane), $X_{s H s}$ is the $x$ location of the horizontal stabilizer measured in the Hub-body coordinate system, and $\beta_{f}$ is the sideslip angle.

$$
\beta_{f}=-\tan ^{-1}\left(\frac{\mu_{y}}{\mu_{x}}\right)
$$

The variation of downwash, normalized with $\frac{10 C_{r} \Omega R}{\mu}$, at the horizontal stabilizer for $\mu=0.22$, given by equation (2.34), is shown in figure 2.14 . It can be seen that for 


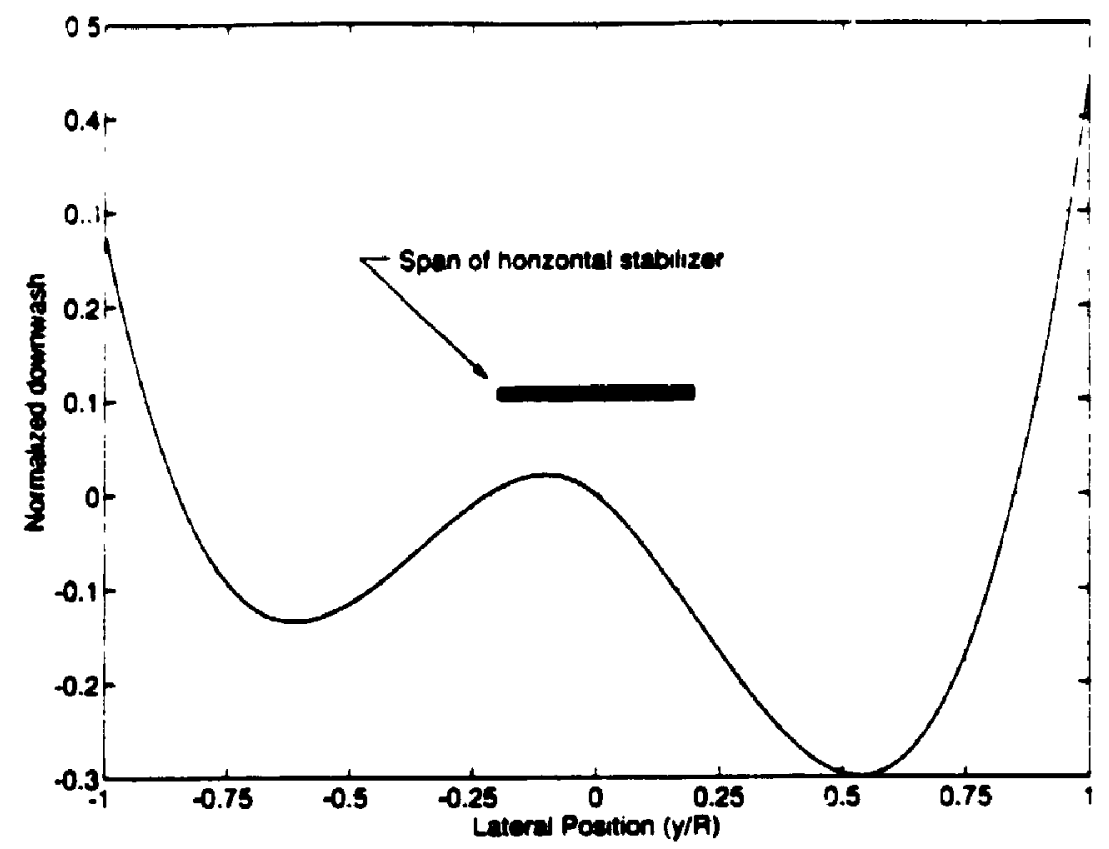

Figure 2.14: Downwash variation for zero sideslip $(\mu=0.22)$

these conditions the left side of the stabilizer experiences upwash whereas the right side experiences downwash resulting in a net rolling moment. As a first order approximation, the terms $w_{i H s_{1}}$ and $w_{i H s_{2}}$ are determined by averaging the downwash over the span of the right and left stabilizers respectively.

\section{Force and Moment Contribution}

The orientation of the lift and drag forces as produced by the horizontal stabilizer are shown in figure 2.15 where $\alpha_{H S}$ is the complex angle of attack and $\beta_{H S}$ is the sideslip. The drag is aligned with the total velocity $V_{H S}$ which makes an angle of $\beta_{H S}$ with the longitudinal plane $\left(\hat{x}_{r}-\hat{z}_{\vec{r}}\right)$ of the helicopter. The lift is also in the longitudinal plane tilted at an angle of $\alpha_{H S}$ from the vertical axis $\dot{z}_{F}$. The horizontal stabilizer contribution to the total force and moment on the helicopter follows from figure 2.15:

$$
\begin{aligned}
& F_{x_{H S_{1,2}}}=Q_{H S}\left(-C_{D_{H S}} \cos \beta_{H S} \cos \alpha_{H S}+C_{L_{H S}} \sin \alpha_{H S}\right)_{1,2} \\
& F_{Y_{H S_{1,2}}}=Q_{H S}\left(-C_{D_{H S}} \sin \beta_{H S}\right)_{1,2} \\
& F_{z_{H S_{1,2}}}=Q_{H S}\left(-C_{L_{H S}} \cos \alpha_{H S}-C_{D_{H S}} \cos \beta_{H S} \sin \alpha_{H S}\right)_{1,2} \\
& M_{x_{H S}}=\left(F_{z_{H S_{1}}}-F_{z_{H S_{2}}}\right) Y_{F H S}+\left(F_{Y_{H S}}+F_{Y_{H S_{2}}}\right) Z_{F H S}
\end{aligned}
$$


CHAPTER 2. MODELING

lis

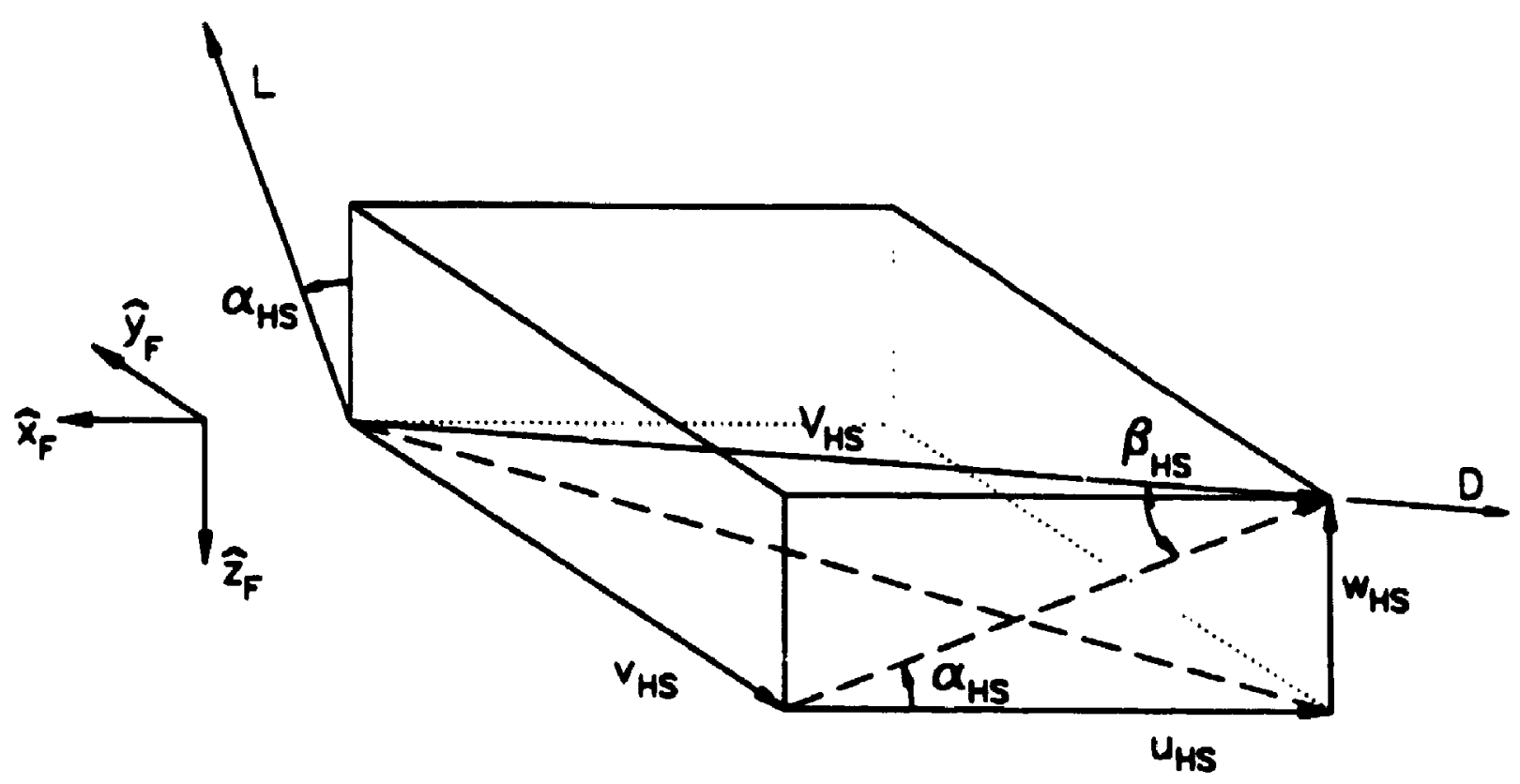

Figure 2.15: Force/Moment geometry at horizontal stabilizer

$$
\begin{aligned}
& M_{y_{H S}}=\left(F_{z_{H S_{1}}}+F_{z_{H S_{2}}}\right) X_{F H S}-\left(F_{I_{H_{H}}}+F_{\Sigma_{Y_{H} S_{2}}}\right) Z_{F H S} \\
& M_{z_{H S}}=-\left(F_{V_{H S}}+F_{Y_{H S_{2}}}\right) X_{F H S}+\left(F_{x_{H S}}-F_{x_{H S} S_{1}}\right) Y_{F H S}
\end{aligned}
$$

where,

$$
\begin{aligned}
Q_{H S}= & \frac{1}{2} \rho V_{H S}^{2} S_{H S} \eta_{H S} \\
S_{H S}= & \text { planform area } \\
\eta_{H S}= & \text { fuselage blockage factor } \\
\alpha_{H S}= & \tan ^{-1}\left(\frac{w_{H S}}{u_{H S}}\right)+i_{H S} \\
i_{H S}= & \text { incidence of stabilizer zero lift line w.r.t. fuselage } \\
& \text { coordinate system, positive nose up } \\
\beta_{H S}= & \sin ^{-1}\left(\frac{v_{H S}}{V_{H S}}\right) \\
V_{H S}= & \sqrt{u_{H S}^{2}+v_{H S}^{2}+w_{H S}^{2}}
\end{aligned}
$$

$F_{x_{H S}}, F_{Y_{H S}}, F_{Y H S}=$ longitudinal, lateral, and vertical forces in the fuselage system $M_{I_{H S}}, M_{Y_{H S}}, M_{2 H S}=$ roll, pitch, and yaw moments in the fuselage system

Finally, the lift curve slope as a function of sideslip is given by:

$$
a_{y s}=a_{0} \cos ^{2} \beta_{H s}
$$


whare 4, is the lift curve slope at zero sideslip. The effect of wing sweep is not considered as the ,weep angle is zero.

\subsubsection{Vertical Fin}

The vertical fin is modeled as a symmetrical wing with sweep. Main rotor downwash affects are accounted for using induced velocity charts.

\section{Velocity}

The center of pressure of the fin is located at $\left\{X_{F V F}, 0, Z_{F V F}\right\}$ in the fuselage coordinate system. The velocity is given by:

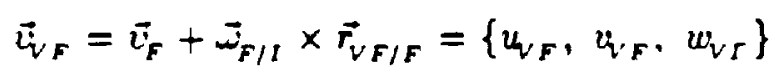

thus.

$$
\begin{aligned}
& u_{v F}=u_{F}-\dot{\theta} Z_{F V F} \\
& v_{V F}=v_{F}-\dot{\psi} X_{F V F}+\dot{\phi} Z_{F V F}+v_{V_{T R}} k_{v_{f}} \\
& w_{V F}=w_{F}+\dot{\theta} X_{F V F}-w_{V F}
\end{aligned}
$$

where $w_{V_{F}}$ is the main rotor downwash defined positive downward and $v_{T R}$ is the tailrotor induced velocity. The tail rotor-fin interaction was accounted for earlier as a thrust reduction. thus the factur $k_{v}$, is left as an unknown to be determined at the model verification stage of the model development. The main rotor downwash is calculated as before using $X / R=1.1$ and $Z / R=-0.21$ :

$$
\frac{w_{w F}}{v_{i}}= \begin{cases}-0.324-3.800 \chi+29.877 \chi^{2}-35.682 \chi^{3}+11.820 \chi^{4} & \beta_{w}=0^{\circ} \\ -0.300-0.437 \chi-0.224 \chi^{2}-0.044 \chi^{3}+0.112 \chi^{4} & \beta_{w}= \pm 90^{\circ} \\ -0.300+0.542 \chi-2.230 \chi^{2}+0.278 \chi^{3}+1.959 \chi^{4} & \beta_{w}=180^{\circ}\end{cases}
$$

\section{Force and Moment Contribution of Vertical Fin}

The orientation of the lift and drag forces produced by the vertical fin are shown in figure 2.16 where $\alpha_{v r}$ is the complex angle of attack and $\beta_{v_{r}}$ is the sideslip. The drag is aligned with the total velocity $V_{V F}$ which makes an angle of $\beta_{V F}$ with the $\dot{x}_{F}-\dot{y}_{F}$ plane, and 
CHAPTER 2. MODELING

$i$
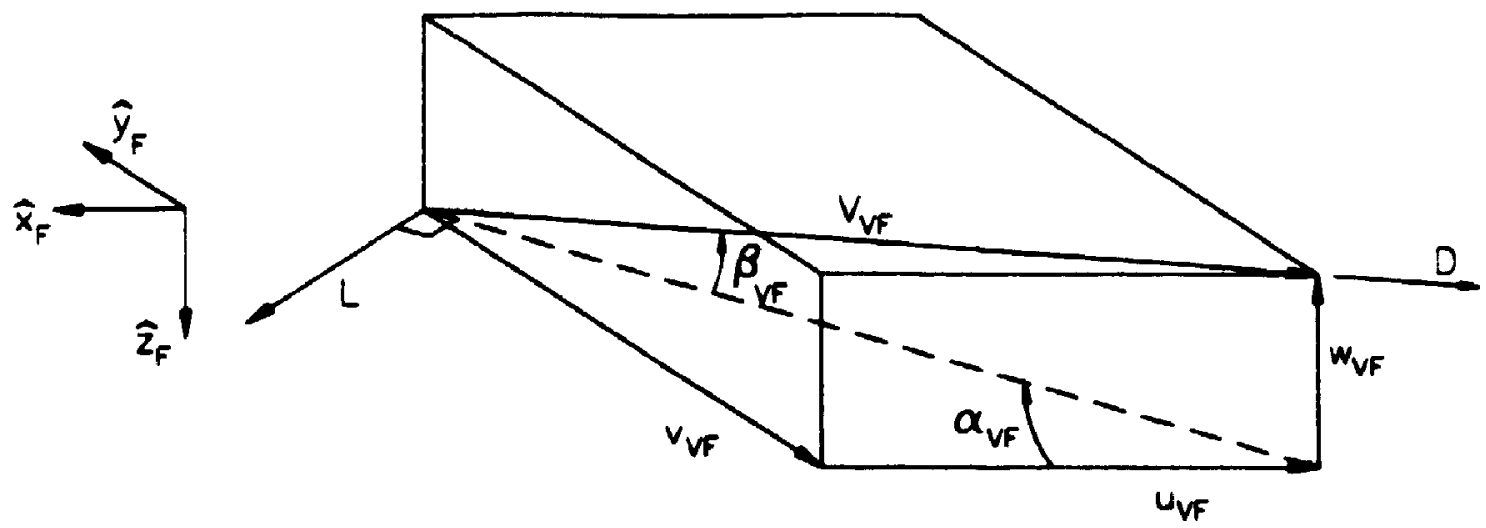

Figure 2.16: Force/Moment geometry at vertical fin

the lift is in this same plane tilted by $\alpha_{q_{F}}$ from the $\dot{y}_{F}$ axis. The vertical fin contribution to the total force and moment on the fuselage can be derived using figure 2.16:

$$
\begin{aligned}
& F_{\bar{\nu}_{F}}=Q_{V_{F}}\left(-C_{Q_{V_{F}}} \cos \beta_{V F} \cos \alpha_{V_{F}}+C_{L_{V_{F}}} \sin \alpha_{V_{F}}\right) \\
& F_{V_{F}}=Q_{V F}\left(-C_{D_{V F}} \cos \beta_{V F} \sin \alpha_{V F}-C_{L_{F}} \cos \alpha_{V_{F}}\right) \\
& F_{i_{F}}=Q_{V F}\left(-C_{Q_{F}} \sin 3_{V_{F}}\right) \\
& M_{V_{V F}}=F_{W_{F}} Z_{F V F} \\
& M_{W_{F}}=-F_{V_{V F}} Z_{F V F}+F_{V_{F}} X_{F V F} \\
& M_{q_{F}}=-F_{W_{F}} X_{F V F}
\end{aligned}
$$

where.

$$
\begin{aligned}
& Q_{V F}=\frac{1}{2} \rho V_{V F}^{2} S_{V F} \eta_{F F} \\
& S_{v f}=\text { platform area } \\
& \eta_{F F}=\text { fuselage blockage factor } \\
& \alpha_{v_{F}}=\tan ^{-1}\left(\frac{v_{V F}}{u_{v_{F}}}\right) \\
& \beta_{v F}=\sin ^{-1}\left(\frac{w_{V F}}{V_{V F}}\right) \\
& V_{V F}=\sqrt{u_{V F}^{2}+v_{V F}^{2}+w_{V F}^{2}} \\
& F_{x_{V F}}, F_{W_{F}}, F_{\psi_{F}}=! \text {; gitudinal, lateral, and vertical forces in the fuselage system } \\
& M_{z_{F}}, M_{W_{F}}, M_{v_{F}}=\text { roll, pitch, and yaw moments in the fuselage system }
\end{aligned}
$$


The lift curve slope modified to account for sideslip and sweep becomes:

$$
a_{V F}=\left(\frac{2 \pi}{1+2 / A R_{V F}}\right) \cos ^{2}\left(\beta_{V F}+L_{V F}\right)
$$

where $\lambda_{v F}$ is the sweep angle of the vertical fin.

\subsection{Fuselage}

The fuselage is modeled for all orientations of the fuselage with respect to the wind. For small angles of attack and sideslip $\left( \pm 15^{\circ}\right)$, wind tunnel test data is employed to obtain a good approximation to the actual characteristics of the fuselage. At high angles of attack and sideslip $\left( \pm 30^{\circ}\right.$ to $\left.\pm 180^{\circ}\right)$, a crude approximation is used due to the lack of data in this flight regime. The importance of the fuselage forces at these high attitudes is diminished by the rotor forces, and thus an accurate model is not necessary. The high and low angle models presented here are taken from reference [27] and [22].

Due to the lack of data, the longitudinal forces and moments are assumed to be a function of angle of attack only and the lateral forces and moments are assumed to be a function of sideslip angle only. However, it is possible to represent the drag forces in terms of both angle of attack and sideslip.

The orientarion of the forces and moments are shown in figure 2.17 where $D, Y$, $L, l, M$, and $N$ are the wind axes drag, sideforce, lift, rolling moment, pitching moment, and yawing moment respectively. The relative wind vector $\vec{V}_{F}$ has the components $\left\{u_{r_{w}}, v_{F_{w}}, w_{r_{w}}\right\}$ in the body axes. The transformation matrix from the wind axes $\left(\hat{x}_{w}, \hat{u}_{w}, \hat{z}_{w}\right)$ to the fuselage coordinate system is given by:

$$
T_{F W}=\left[\begin{array}{ccc}
-\cos \alpha_{F} \cos \beta_{F} & -\cos \alpha_{F} \sin \beta_{F} & \sin \alpha_{F} \\
\sin \beta_{F} & \cos \beta_{F} & 0 \\
-\sin \alpha_{F} \cos \beta_{r} & -\sin \alpha_{F} \sin \beta_{F} & -\cos \alpha_{F}
\end{array}\right]
$$

where $\alpha_{F}$ is the complex angle of attack and $\beta_{F}$ is the sideslip angle defined according to:

$$
\begin{aligned}
& \alpha_{r}=\tan ^{-1}\left(\frac{w_{r_{w}}}{u_{r_{w}}}\right) \\
& \beta_{r}=\sin ^{-1}\left(\frac{v_{r_{v}}}{V_{r}}\right)
\end{aligned}
$$




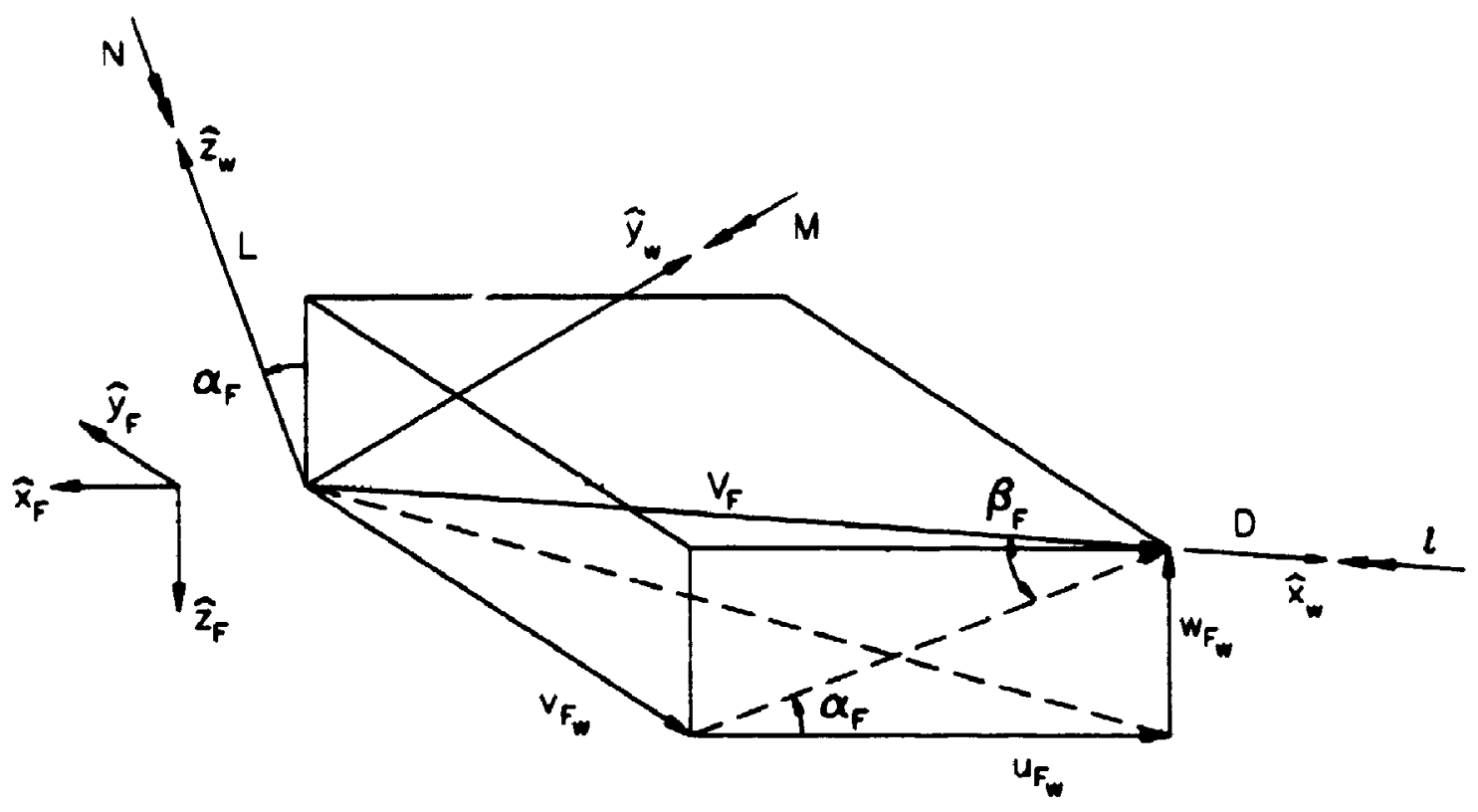

Figure 2.1i: Force/Moment geometry at fuselage

where,

$$
\begin{aligned}
u_{F_{W}} & =u_{F} \\
v_{F_{W}} & =v_{F} \\
w_{F_{\omega}} & =w_{F}-w_{i_{F}} \\
v_{F} & =\sqrt{u_{F_{W}}^{2}+v_{F_{W}}^{2}+w_{F_{\omega}}^{2}}
\end{aligned}
$$

The main rotor downwash at the fuselage aerodynamic reference point, $w_{F_{F}}$, is calculated as before by way of induced velocity charts. However, since the longitudinal location ", the fuselage aerodynamic reference point lies at $X_{I} R \approx 0$, the need for induced velocity modeling as a function of sideslip is eliminated. In addition, the induced velocity at a single point in the main rotor wake can not be used to determine the main rotor interference on the entire fuselage as was done with the empennage surfaces and the tail rotor. Therefore, the main rotor downwash at the fuselage is determined with the induced velocity charts for unifrrm disk loading. The uniform disk loading is chosen instead of the triangular disk loading because it provides an averaging effect. The main rotor downwash at the fuselage is thus:

$$
\frac{w_{i_{5}}}{v_{1}}=1.291-0.544 x+1.765 \chi^{2}-1.420 \chi^{3}+0.182 \chi^{4}
$$


The lift. drag. and moment expressions for the small angle model (SA.M) are given by:

$$
\begin{aligned}
L & =q_{F}\left(\frac{L_{0}}{q}+\frac{\partial L / q}{\partial \alpha} \alpha\right) \\
Y & =q_{F}\left(\frac{\partial Y / q}{\partial 3} 3\right) \\
D_{\alpha} & =q_{F}\left(\frac{D_{0}}{q}+\frac{\partial D}{\partial \alpha} \alpha+\frac{\partial^{2} D / q}{\partial \alpha^{2}} \alpha^{2}\right) \\
D_{3} & =q_{F}\left(\frac{\partial^{2} D / q}{\partial 3^{2}} 3^{2}\right) \\
D & =D_{\alpha}+D_{3} \\
l & =q_{F}\left(\frac{\partial l / q}{\partial 3} 3\right) \\
M & =q_{F}\left(\frac{M_{0}}{q}+\frac{\partial M / q}{\partial \alpha} \alpha\right) \\
v & =q_{F}\left(\frac{\partial N / q}{\partial 3} 3\right) \\
q_{F} & =\frac{1}{2} \rho V_{F}^{2}
\end{aligned}
$$

where $L_{o}, D_{o}$, and.$H_{o}$ are the lift, drag, and pitching moment for zero angle of attack and sideslip.

The high angle model (HA.M) makes use of drag data at an angle of attack and sideslip of $90^{\circ}$. If the angle of attack is $90^{\circ}$ then the drag force could be expressed as:

$$
D=q_{r}\left(\frac{D_{a=90^{\circ}}}{q}\right)
$$

and the lift could be assumed to be negligible. For angles of attack different from $90^{\circ}$ it is possible to find the portion of $q_{F}$ which contributes to $D_{\alpha=90^{\circ}}$ as shown in figure 2.18 (a):

$$
\begin{aligned}
q_{F_{a=90^{\circ}}} & =q_{F} \sin ^{2} \alpha \\
\mathcal{D}_{\alpha} & =q_{F}\left(\frac{D_{\alpha=90^{\circ}}}{q} \sin ^{2} \alpha\right)
\end{aligned}
$$

where $\sin ^{2} \alpha$ has been used for the $V_{F}^{2}$ term in $q_{F_{0=000}}$. The portion of $q_{F}$ which contributes to $L_{o}$ has been assumed to be small when compared to the drag. Then, the lift and drag variation with angle of attack can be expressed as:

$$
L=\mathcal{D}_{\alpha} \cos \alpha=q_{F}\left(\frac{D_{\alpha=90^{\circ}}}{q} \cos \alpha|\sin \alpha| \sin \alpha\right)
$$




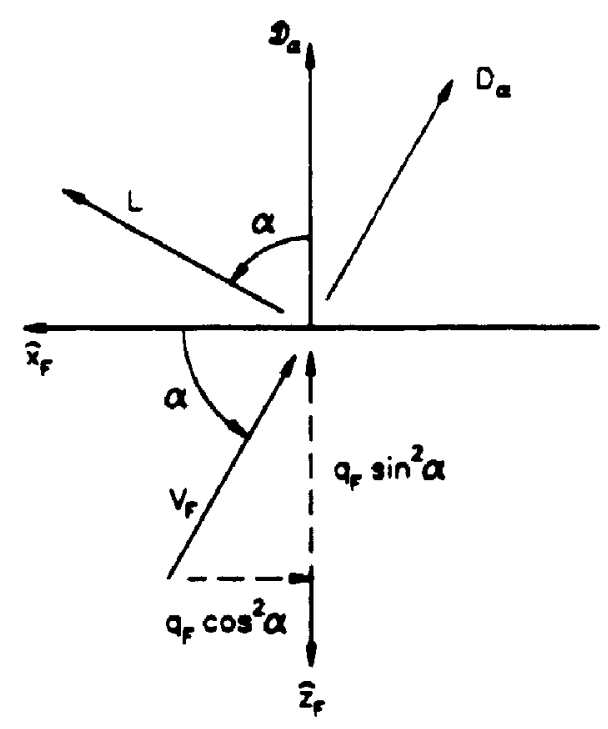

(a)

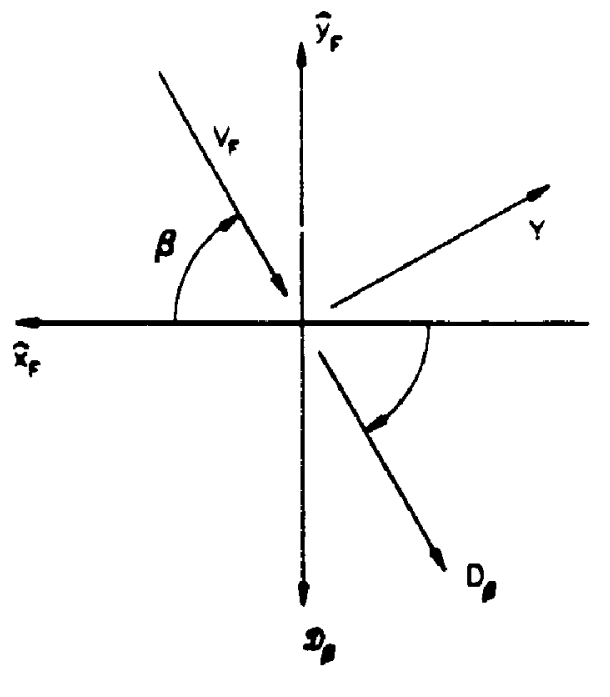

(b)

Figure 2.18: Fuselage models

$$
D_{\alpha}=\mathcal{D}_{\alpha} \sin \alpha=q_{F}\left(\frac{D_{\alpha=90^{\circ}}}{q} \sin ^{2} \alpha|\sin \alpha|\right)
$$

where the form of the expression has been chosen to give the correct sign of lift and drag $(L-+v e$ for $\alpha \rightarrow+v e, L \rightarrow-v e$ for $\alpha \rightarrow-v e, D \rightarrow+v e$ ). The same reasoning can be used or sideslip and $\mathrm{Y}$ force as shr $\mathrm{n}$ in figure 2.18 (b):

$$
\begin{aligned}
D_{\beta} & =\mathcal{D}_{\beta} \sin \beta=q_{F}\left(\frac{D_{\beta=90^{\circ}}}{q} \sin ^{2} \beta|\sin \beta|\right) \\
Y & =\mathcal{D}_{\beta} \cos \beta=q_{F}\left(\frac{-D_{\beta=90^{\circ}}}{q}|\sin \beta| \sin \beta \cos \beta\right)
\end{aligned}
$$

The form of the rolling, pitching, and yawing moments are different form those of lift, drag, and sideforce. Their variation with attitude have been modified to match wind-tunnel tests of bodies of revolution:

$$
\begin{aligned}
l & =q_{F}\left(\frac{l_{B=90^{\circ}}}{q}|\sin \beta| \sin \beta\right) \\
M & =q_{F}\left(\frac{M_{\alpha=90^{\circ}}}{q}|\sin \alpha| \sin \alpha\right) \\
N & =q_{F}\left(\frac{N_{B=90^{\circ}}}{q}|\sin \beta| \sin \beta\right)
\end{aligned}
$$

At attitudes between the low and high angle models a complex phase angle is used to phase one model in while phasing the other model out in order to form a smooth transition 


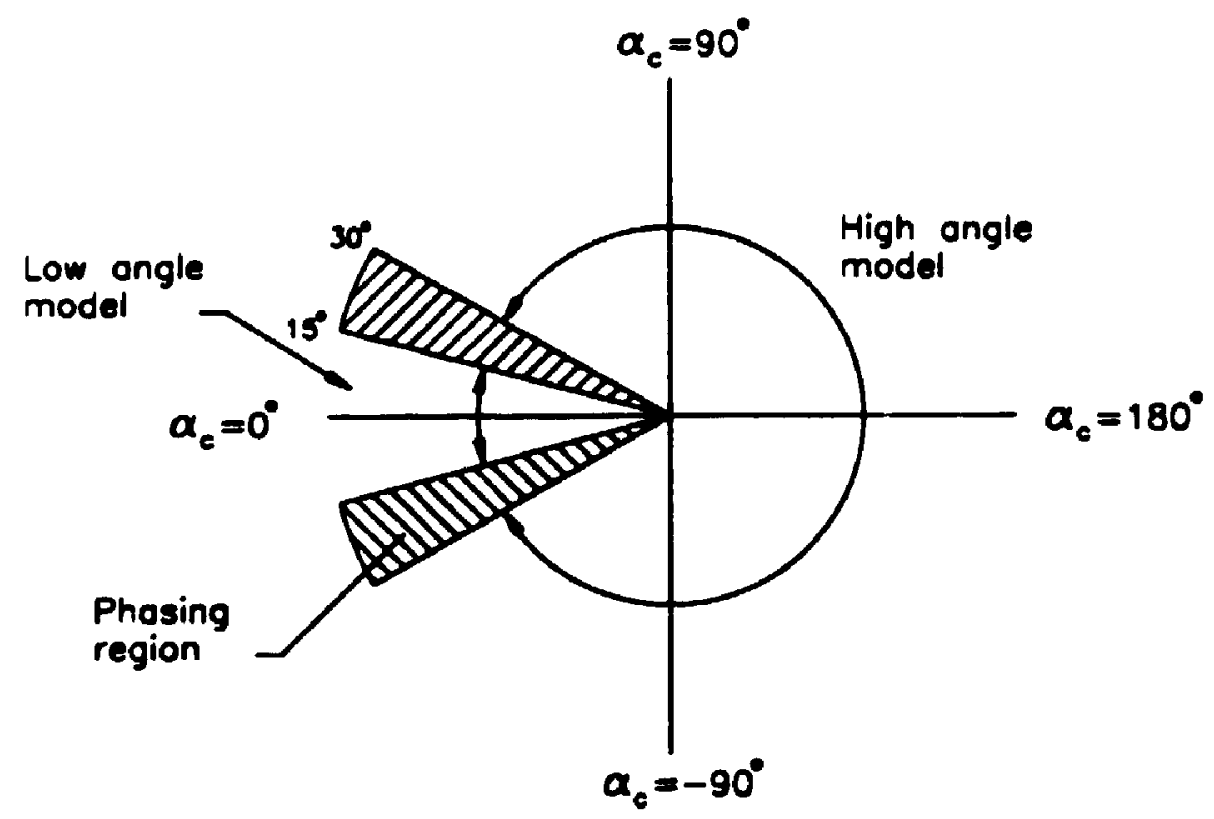

Figure 2.19: Phasing between low and high angle models

between the models. This helps avoid sudden accelerations which are not representative of the flight characteristics of the Bell 205. The complex phase angle, $\alpha_{c}$, defined as the angle between the wind vector, $\vec{V}_{F}$, and the positive body axis, $\dot{x}_{F}$, is given by:

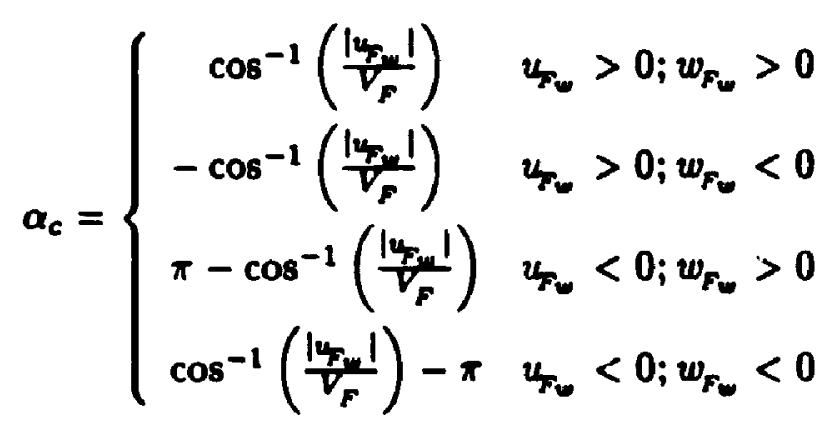

This angle has been chosen over the fuselage angle of attack because it depends on both angle of attack and sideslip of the fuselage. Figure 2.19 shows the phasing region on the complex phasing plane. In the phasing region, linear interpolation is used to calculate a phase angle:

$$
\alpha_{p h}=\left(\frac{\alpha_{c}-15}{30-15}\right) \frac{\pi}{2}
$$

where $\alpha_{c}$ is in degrees and $\alpha_{p h}$ is the phase angle in radians used as input to the force and moment equations according to:

$$
F=F_{L A M} \cos ^{2} \alpha_{p h}+F_{H A M} \sin ^{2} \alpha_{p h}
$$


where

$$
\begin{aligned}
F & =\text { Fuselage force or moment } \\
F_{\text {LAM }} & =\text { Fuselage force or moment from LAM } \\
F_{H A M} & =\text { Fuselage force or moment from HAM }
\end{aligned}
$$

The fuselage contribution to the total forces on the helicopter are:

$$
\left\{\begin{array}{l}
F_{x_{F}} \\
F_{y_{F}} \\
F_{z_{F}}
\end{array}\right\}=T_{F W}\left\{\begin{array}{l}
D \\
Y \\
L
\end{array}\right\}=\left\{\begin{array}{l}
-D \cos \alpha_{F} \cos \beta_{F}-Y \cos \alpha_{F} \sin \beta_{F}+L \sin \alpha_{F} \\
-D \sin \beta_{F}+Y \cos \beta_{F} \\
-D \sin \alpha_{F} \cos \beta_{F}-Y \sin \alpha_{F} \sin \beta_{F}-L \cos \alpha_{F}
\end{array}\right\}
$$

and the contribution to helicopter moments are given by:

$$
\begin{aligned}
\left\{\begin{array}{l}
M_{x} \\
M_{y} \\
M_{z}
\end{array}\right\} & =\left\{\begin{array}{lll}
-1 & 1 & -1\} T_{F} w \\
M \\
-N
\end{array}\right\} \\
& =\left\{\begin{array}{c}
-l \\
l \cos \alpha_{F} \cos \beta_{F}-M \cos \alpha_{F} \sin \beta_{F}-N \sin \alpha_{F} \\
l \sin \beta_{F}+M \cos \beta_{F} \\
l \sin \alpha_{F} \cos \beta_{F}-M \sin \alpha_{F} \sin \beta_{F}+N \cos \alpha_{F}
\end{array}\right\}
\end{aligned}
$$

\subsection{Fuselage eyuations of motion}

The fuselage equations are derived using a Newtonian approach where the linear momentum obeys:

$$
\vec{F}=\frac{d}{d t}\{m \vec{V}\}=m\left(\left(\dot{\vec{V}}_{r}\right)+\vec{w} \times \vec{V}\right)
$$

and the angular momentum obeys:

$$
\vec{M}_{a}=\frac{d}{d t} \vec{H}=(\dot{\vec{H}})_{r}+\vec{\omega} \times \vec{H}
$$

where,

$$
\begin{aligned}
\vec{F} & =\text { sum of all external forces } \\
\vec{M}_{0} & =\text { sum of all external moments } \\
\vec{H} & =\text { angular momentum } \\
m & =\text { mass } \\
\vec{V} & =\text { absolute velocity } \\
\vec{w} & =\text { absolute angular velocity }
\end{aligned}
$$


Issuming that the mass of the body is constant and the earth coordinate system (I) is inertial. equation $(2.35)$ gives:

$$
\begin{aligned}
\vec{F}=\left\{\begin{array}{c}
F_{x} \\
F_{y} \\
F_{z}
\end{array}\right\} & =m\left(\dot{u}_{F} \dot{x}_{F}+\dot{i}_{F} \dot{y}_{F}+\dot{u}_{F} \dot{z}_{F}+\vec{w}_{F} \times \vec{v}_{F}\right) \\
& =m\left[\begin{array}{c}
\dot{u}_{F}+q w_{F}-r v_{F} \\
\dot{v}_{F}+r u_{F}-p w_{F} \\
\dot{u}_{F}+p v_{F}-q u_{F}
\end{array}\right]
\end{aligned}
$$

where,

$$
\begin{aligned}
& F_{x}=F_{x_{M R}}+F_{x_{T R}}+F_{x_{H S}}+F_{z_{Y F}}+F_{x_{F}}+F_{y_{x}} \\
& F_{y}=F_{y_{M R}}+F_{y_{T R}}+F_{y_{H S}}+F_{\psi_{F}}+F_{y_{F}}+F_{g_{y}} \\
& F_{z}=F_{z_{M R}}+F_{\tau_{T R}}+F_{z_{H S}}+F_{z_{y F}}+F_{z_{F}}+F_{g_{z}} \\
&\left\{\begin{array}{c}
F_{y_{x}} \\
F_{g_{y}} \\
F_{g_{x}}
\end{array}\right\}=T_{F I}\left\{\begin{array}{c}
0 \\
0 \\
m g
\end{array}\right\}=\left\{\begin{array}{l}
-m g \sin \Theta \\
m g \cos \Theta \sin \Phi \\
m g \cos \Theta \cos \Phi
\end{array}\right\}
\end{aligned}
$$

The rotational equations of motion follow from equation (2.36):

$$
\vec{M}_{G}=\left\{\begin{array}{c}
M_{x} \\
M_{y} \\
M_{z}
\end{array}\right\}=\left\{\begin{array}{l}
I_{x x} \dot{p}-I_{x z}(\dot{r}+p q)+\left(I_{z z}-I_{y y}\right) q r \\
I_{y y} \dot{q}+\left(I_{x x}-I_{z z}\right) p r-I_{x z}\left(r^{2}-p^{2}\right) \\
I_{z z} \dot{r}+\left(I_{y y}-I_{x x}\right) p q-I_{x z}(\dot{p}-q r)
\end{array}\right\}
$$

where,

$$
\begin{aligned}
& I_{x y}=I_{y z}=0 \text { (due to symmetry) } \\
& M_{x}=M_{x_{M R}}+M_{x_{T R}}+M_{x_{H S}}+M_{x_{Y F}}+M_{x_{F}} \\
& M_{y}=M_{y_{M R}}+M_{y_{T R}}+M_{y_{H S}}+M_{Y_{F F}}+M_{y_{F}} \\
& M_{z}=M_{z_{M R}}+M_{\tau_{T R}}+M_{z_{H S}}+M_{z_{F}}+M_{z_{F}}
\end{aligned}
$$

The rate of change of the Euler angles can be derived using figure 2.2 where it is shown that:

$$
p=\dot{\phi}-\dot{\Psi} \sin \theta
$$




$$
\begin{aligned}
& q=\dot{\Theta} \cos \Phi+\dot{\Psi} \cos \Theta \Phi \\
& r=-\dot{\Theta} \sin \Phi+\dot{\Psi} \cos \Theta \cos \Phi
\end{aligned}
$$

Solving.

$$
\begin{aligned}
\dot{\Phi} & =p+\tan \Theta(r \cos \Phi+q \sin \Phi) \\
\dot{\Theta} & =q \cos \Phi-r \sin \Phi \\
\dot{\Psi} & =\sec \Theta(r \cos \Phi+q \sin \phi)
\end{aligned}
$$

\subsection{Summary}

The nonlinear equations of motion of a teetering rotor helicopter were developed with the fuselage modeled as one rigid body and the spinning rotor as another rigid body that could be tilted with respect to the fuselage by lateral and longitudinal cyclic control or as a result of aerodynamic loads (this model is often called the Rotor Body Model (RB.M)). By deriving all rotor equations in the fuselage axes it was possible to avoid explicit angle calculations which have been documented to cause problems during hovering flight. Quasi-steady aerodynamics was used to calculate the air-loads on the blades and non-linear dynamic inflow theory was used to describe the induced velocity distribution. The aerodynamic loads on the empennage surfaces and the loads and moments on the fuselage were approximated for all angles of attack and sideslip. 


\section{Chapter 3}

\section{Computer Simulation}

The computer program developed is a non-real time helicopter simulator which propagates the state vector in time from a vector of initial conditions. State feedback control is used to stabilize the otherwise unstable helicopter, allowing various maneuvers to be performed. Routines are provided which generate the trim states of the helicopter at desired speeds and also the corresponding linear perturbation models which are intended for control synthesis. The program is written in a modular fashion and can easily be modified to simulate various single rotor helicopters. Further description of the computer software may be found in appendix $C$.

\subsection{Integration Method}

For a non-real time helicopter simulation Davis ${ }^{[35]}$ shows that the fourth-order RungeKutta (RK4) method provides good accuracy as well as good stability characteristics for a fairly large integration step size. RK4 is not very efficient but its simplicity makes it a good first choice.

The routine chosen for the simulation is a fourth-order Runge-Kutta method which uses step doubling to provide adaptive step size control and truncation error correction. The desired accuracy for each equation can be chosen independently of the others; the routine will choose a step size to accommodate the "worse" equation. Details of the integration algorithm can be found in [36].

An important concern in helicopter simulation is the dynamic accuracy of the integration scheme. That is, how accurately does the dynamics of the solution match that of the 
true solution. It is important to choose a step size that "captures" the proper dynamics of all important modes of the helicopter. One way to achieve this is to take the mode with the highest frequency and use the analysis of $[3 i]$ to pick a step size to achieve a desired dynamic accuracy.

Howe ${ }^{[38]}$ gives the damping ratio and fractional frequency errors in simulating an under. damped second order system using an RKt method as:

$$
\begin{aligned}
& \dot{\zeta}-\dot{\zeta} \cong \quad \frac{2}{9}\left(\omega_{n} T\right)^{5} \\
& \frac{\dot{y}_{d}}{\omega_{d}}-1 \cong
\end{aligned}
$$

where $\dot{\zeta}$ and $\dot{\psi}_{\dot{1}}$ are the damping ratio and damped natural frequency obtained by use of an RK4 method ( $\left.\omega_{n} T \ll 1\right)$. Similarly, $\zeta$ and $\omega_{d}$ are the exact damping ratio and damped natural frequency respectively. $T$ is the integration interval and $\omega_{n}$ is the undamped natural frequency of the system. Equation (3.1) can be used to calculate a required integration interval for a desired accuracy in $\zeta$ and $\omega_{d}$.

As discussed earlier the highest frequency mode of the helicopter is that associated with the main rotor flapping, namely the advancing flapping mode. For the Bell 205 the advancing mode can be expected to have an undamped natural frequency of $65 \mathrm{rad} / \mathrm{s}$, a damped natural frequency of $63.7 \mathrm{rad} / \mathrm{s}$, and a damping ratio of 0.21 . Thus if an accuracy of $0.1 \%$ is $s t_{2}$ dired in frequency, the integration interval must be chosen smaller than or equal to 0.0045 seconds. This results in a damping ratio error of $\dot{\zeta}-\zeta=0.00049$. Since the routine chosen adjusts its own step size it is only necessary to limit the maximum step size to 0.004 seconds.

The analysis of reference [33] is geared towards linear systems and can not be expected to give accurate results for a system as complex as a helicopter; however, it provides a good starting point.

\subsection{Stabilization}

A stability augmentation system in the form of simple state feedback is used to stabilize the otherwise unstable dynamics of the helicopter. The uncontrolled helicopter possesses a few unstable modes, some of which are stabilized in forward flight but nevertheless require external control to increase damping and aid performance. The most common type of feedback control law used to stabilize a helicopter is a simple attitude/rate feedback of 
the form:

$$
\begin{aligned}
A_{s} & =k_{\theta} \theta+k_{p} q \\
A_{s} & =k_{p} \theta+k_{p} p \\
\theta_{M R} & =k_{u_{F}} u_{F} \\
\theta_{T R} & =k_{r} r
\end{aligned}
$$

where the feedback gains have been given the symbol $k_{1}$. The vertical and yaw channels of the helicopter behave like an over-damped second order system (in most flight regimes) and do not require stabilization. Feedback laws (3.4) and (3.5) are oniy used to alter the response time of the aircraft.

The rate-attitude feedback control laws are sufficient to stabilize a piloted helicopter. however, for a computer simulation some form of velocity control is also necessary to control translational motion. The final form of the control law used airws direct cortrol over the velocity of the helicopter:

$$
\begin{aligned}
& A_{g}=k_{\theta} \theta+k_{q} q+k_{s e} \dot{x}_{e}+k_{e s} \int\left(\dot{x}_{e}-\dot{x}_{r e f}\right) d t \\
& A_{c}=k_{\phi} \phi+k_{p} p+k_{v e} \dot{y}_{e}+k_{e c} \int\left(\dot{y}_{e}-\dot{y}_{r e f}\right) d t \\
& \theta_{M R}=k_{h} \dot{h}+k_{e m r} \int\left(\dot{h}-\dot{h}_{r e f}\right) d t \\
& \theta_{\tau R}=k_{r} r+k_{\psi} \psi+k_{i} \dot{r}
\end{aligned}
$$

where $\left(\dot{x}_{e}, \dot{y}_{e},-\dot{h}\right)$ are earth velocities defined according to:

$$
\left\{\begin{array}{c}
\dot{x}_{e} \\
\dot{y}_{e} \\
-\dot{h}
\end{array}\right\}=T_{F I}^{\mathrm{T}}\left\{\begin{array}{c}
u_{F} \\
v_{F} \\
w_{F}
\end{array}\right\}
$$

\subsubsection{The Bell Stabilizing Bar}

The stabilizing bar, fig. 3.1, is a mechanical stability augmentation system. It is a bar connected to the main rotor shaft via a single hinge which allows it to change its orientation with respect to the shaft. The bar is viscously damped against rotation relative to the shaft and is connected to the rotor blade feathering mechanism in such $a$ way that a change of orientation with respect to the shaft results in a change in blade pitch. The purpose of the bar is to provide a time lagged feedback of rotor-mast angular rates in pitch and roll; the time lag being a result of bar inertia and viscous damping. 


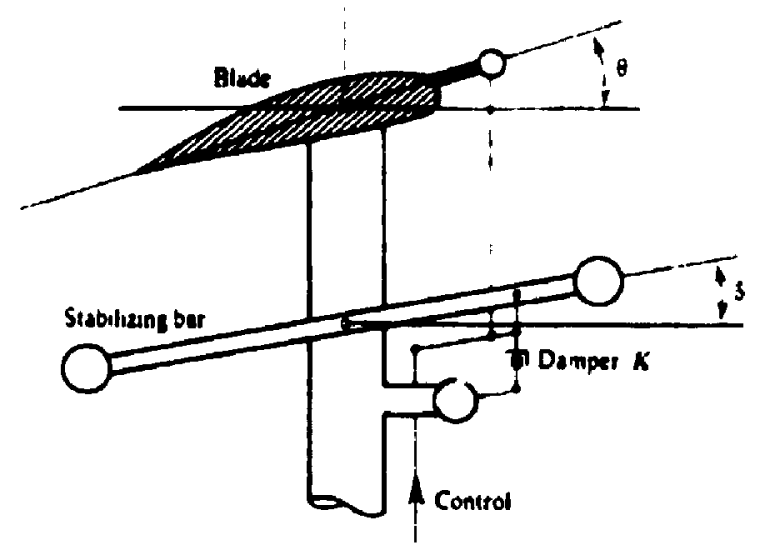

Figure 3.1: Schematic of the Bell Stabilizer Bar ${ }^{[25]}$

The detailed equations of motion for the bar can be found in [25]. If the high frequency mode of the bar, which has a negligible effect on the helicopter, is ignored, the resulting equations become $e^{(25)}$ :

$$
\begin{aligned}
& \frac{d\left(\Delta A_{s}\right)}{d t}=-\frac{1}{T_{f}} \Delta A_{s}+c_{s} q \\
& \frac{d\left(\Delta A_{c}\right)}{d t}=-\frac{1}{T_{f}} \Delta A_{c}-c_{s}\left(p \cos i_{m}\right)
\end{aligned}
$$

where $T_{f}$ is the time constant of the system and $c_{g}$ is the bar to blade linkage factor $\frac{\theta}{q}$ (for the Bell $205 T_{f}=3 \mathrm{sec}, c_{s}=0.16^{[3]}$ ). The schematic of the control law is shown in figure 3.2 and the resulting pitch, roll, and forward velocity responses to a one second longitudinal step input are shown in figures 3.3, and 3.4. The stabilizing effect of the bar is clearly evident.

\subsection{Trim Point Solution}

The usual approach for finding the trim point is to use classical regression analysis to manipulate the controls until the state derivative vector $\left\{\ddot{\theta}_{R}, \dot{\theta}_{R}, \ddot{\phi}_{R}, \dot{\phi}_{R}, \dot{p}, \dot{q}, \dot{r}, p, q, r, \dot{u}, \dot{i}\right.$. $\left.\dot{w}, \dot{\lambda}_{0}, \dot{\lambda}_{s}, \dot{\lambda}_{c}, \dot{v}_{i_{R}}\right\}$ is sufficiently close to zero. This procedure was only performed once with a simplified version of . he equations using Mathematica. Trim points are now found by "flying" the helicopter to the desired point with the control laws described earlier. Possible draw backs of this approach are the increased computer time required to fly from 

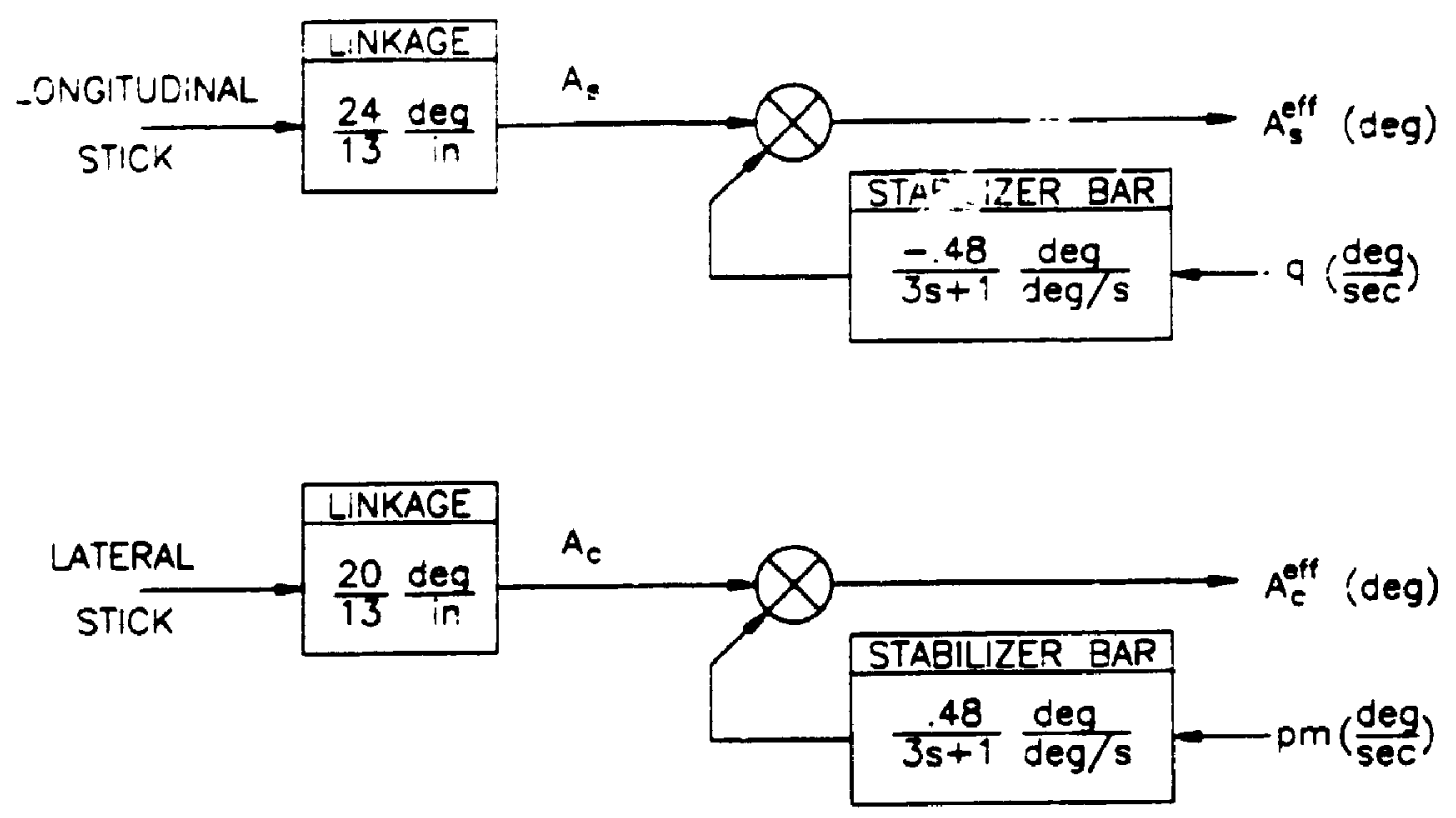

Figure 3.2: Schematic of the Bell st.2bilizer bar control law
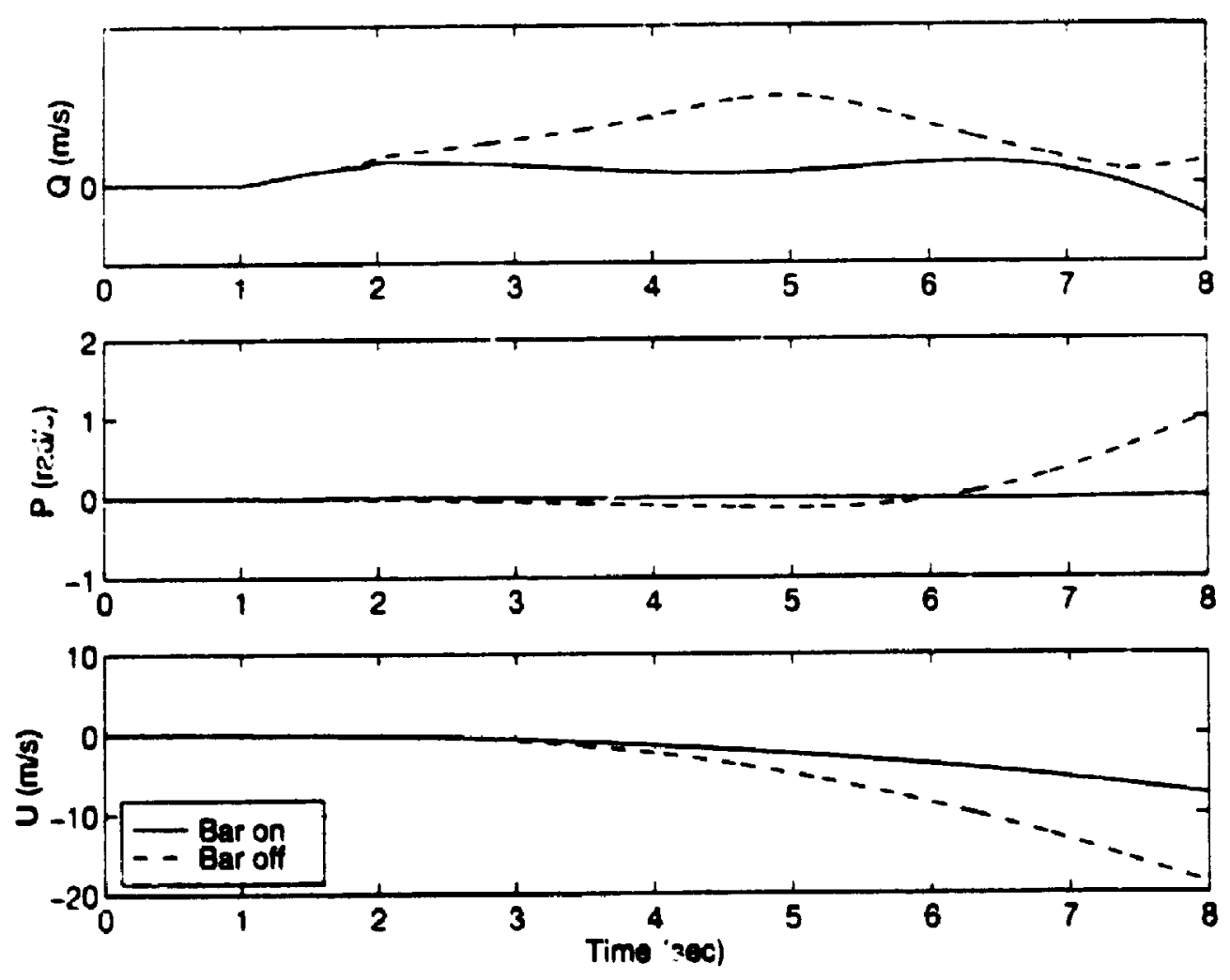

Figure 3.3: Response to a one second step input at hover 

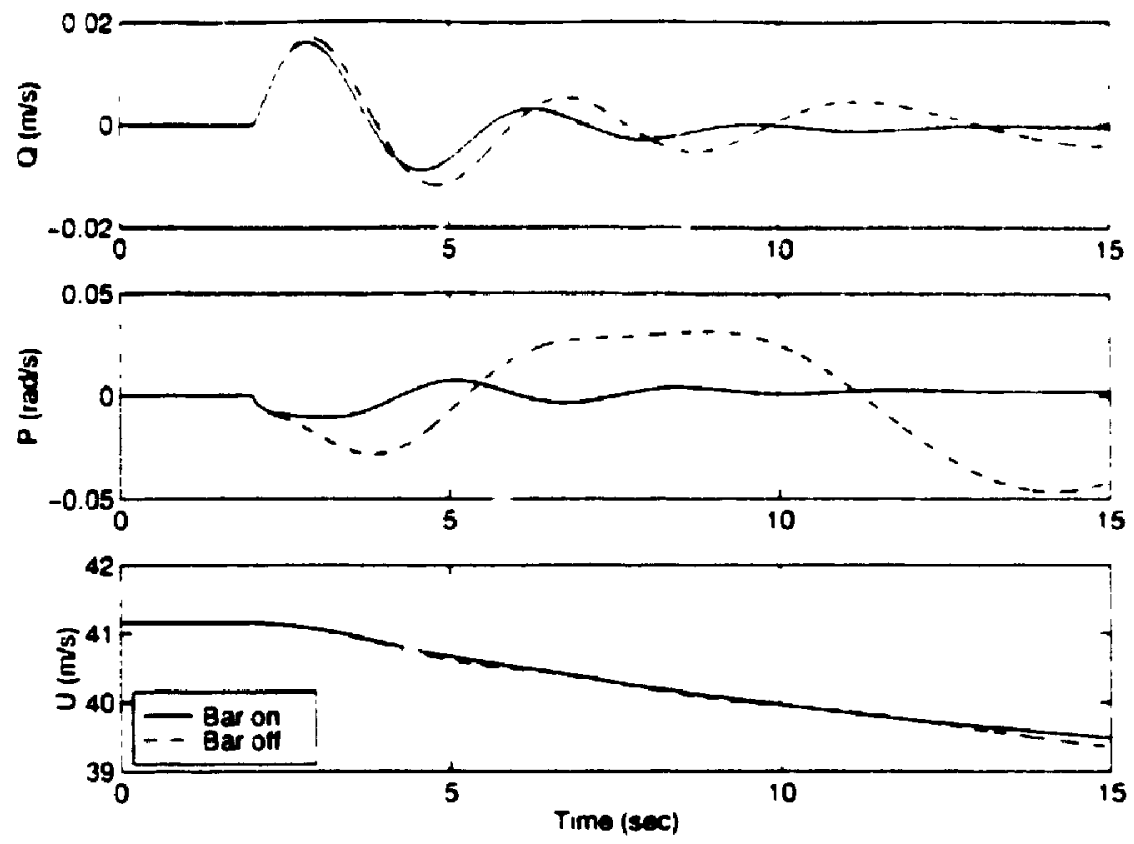

Figure 3... Response to a step input at 80 knots

one state to another and also possible control instabilities introduced by altering propertias of the helicopter.

A linear regression trimming routine can easily be added in the future if the need arises.

\subsection{Linearization}

For the purposes of stability and control analysis it is desired to have linea- mall perturbatiun) models about appropriate trim points. The linear model can be capressed as:

$$
\dot{\hat{x}}=A \dot{x}+B \dot{u}
$$

where $\hat{x}$ represents the perturbation of the state vector from trim and $\dot{u}$ the perturbation of the control vect - from trim. Expressing the nonlinear aircraft model as:

$$
\begin{aligned}
& \dot{\bar{x}}=\bar{f}(\bar{x}, \bar{u}, t) \\
& \bar{x}_{\text {TRIM }}=\bar{x}_{O} \\
& \bar{u}_{\text {TRIM }}=\bar{u}_{O}
\end{aligned}
$$


thasl.

$$
\dot{x}=\bar{x}(t)-\bar{x}_{0}(t)
$$

Substituting expression (3.8) into (3.7) and expanding in a Iaylor series:

$$
\dot{\hat{x}}_{o}+\dot{\dot{x}}=\bar{f}\left(\bar{x}_{0}, \bar{u}_{o} t\right)+\left[\frac{\partial \bar{f}}{\partial \bar{x}}\right]_{0} \dot{\boldsymbol{x}}+\left[\frac{\partial \bar{f}}{\partial \bar{u}}\right]_{0} \dot{u}+\text { h.o.t. }
$$

Ignoring the higher order terms and noting that the trim solution to $(3 . \overline{)})$ satisfies $\dot{\bar{x}}_{0}=$ $\bar{f}\left(\dot{x}_{o}, \bar{u}_{o}, t\right)$ :

$$
\dot{\dot{x}}=\left[\frac{\partial \bar{f}}{\partial \bar{x}}\right]_{0} \dot{x}+\left[\frac{\partial \bar{f}}{\partial \bar{u}}\right]_{0} \dot{u}
$$

where $\left[l_{0}\right.$ denotes evaluation at the trim point. The $A$ and $B$ matrices may now be defined acce ding to:

$$
\begin{aligned}
& A=\left[\begin{array}{ccc}
\frac{\partial f_{1}}{\partial x_{1}} & \cdots & \frac{\partial f_{1}}{\partial x_{n}} \\
\vdots & \ddots & \vdots \\
\frac{\partial f_{n}}{\partial x_{1}} & \cdots & \frac{\partial f_{n}}{\partial x_{n}}
\end{array}\right]_{n \times n} \\
& B=\left[\begin{array}{ccc}
\frac{\partial f_{2}}{\partial u_{1}} & \cdots & \frac{\partial f_{1}}{\partial u_{m}} \\
\vdots & \ddots & \vdots \\
\frac{\partial f_{n}}{\partial u_{1}} & \cdots & \frac{\partial f_{n}}{\partial u_{m}}
\end{array}\right]_{n \times m}
\end{aligned}
$$

The partial derivatives may be approximated by the first-central difference formula:

$$
\frac{\partial f_{i}}{\partial x_{j}}=\frac{f_{1}\left(x_{30}+\Delta x_{\jmath}\right)-f_{1}\left(x_{30}-\Delta x_{\jmath}\right)}{2 \Delta x_{3}}
$$

where,

$$
\begin{aligned}
f_{1} & =\mathrm{i}^{\text {th }} \text { function in (3.7) } \\
x_{j_{0}} & =\text { trim value of } \mathrm{j}^{\text {th }} \text { state } \\
\Delta_{3} & =\text { perturbation in } \mathrm{j}^{\text {th }} \text { state }
\end{aligned}
$$

The perturbations $\Delta x$, are constant values and we chosen according to:

$$
\begin{aligned}
& \Delta \bar{u}=(\Delta u, \Delta v, \Delta w) \quad=0.01 \mathrm{~m} / \mathrm{s} \\
& \Delta \bar{p}=\left(\Delta p, \Delta q, \Delta r, \Delta \dot{\theta}_{R}, \Delta \dot{\phi}_{R}\right)=5 \mathrm{deg} / \mathrm{s} \\
& \Delta \bar{\phi}=\left(\Delta \phi, \Delta \theta, \Delta \psi, \Delta \phi_{R}, \Delta \theta_{R}\right)=1.0 \mathrm{deg} \\
& \Delta \bar{\lambda}=\left(\Delta \lambda_{0}, \Delta \lambda_{0}, \Delta \lambda_{c}\right)=\frac{0.3040}{n R} \\
& \left(\Delta v_{i T R}\right)=\quad=0.05 \mathrm{~m} / \mathrm{s} \\
& \Delta \bar{\delta}=\left(\Delta \theta_{q_{R R}}, \Delta A_{g}, \Delta A_{c}, \Delta \theta_{Q_{T R}}\right)=0.1 \mathrm{in}=0.003222 \mathrm{rad}
\end{aligned}
$$




\subsubsection{Model Reduction}

In some instances it may be desired to obtain a lower order model for analysis or comparison purposes. This can easily be accomplished by simple matrix manipulation as show"I in the following example where the KBM model is reduced to a model with only rigid body dynamics (i.e. the QSM model). The classic 6 D.O.F. quasi-static model treats the helicopter as a single rigid body with "instantaneous" rotor tilting and inflow changes. For this model, the tilt of the rotor and cyclic pitch changes are identical $\left(A_{c}=-\phi_{R}, A_{s}=\theta_{R}\right)$.

The linearized RBM model is partitioned according to:

$$
\begin{aligned}
& \dot{\bar{x}}_{B}=A_{B} \bar{x}_{B}+A_{B R} \bar{x}_{R}+B_{B} \bar{u} \\
& \dot{\bar{x}}_{R}=A_{R B} \bar{x}_{B}+A_{R} \bar{x}_{R}+B_{R} \bar{u}
\end{aligned}
$$

where,

$$
\begin{aligned}
\bar{x}_{B}= & {[u w q v p r \phi \theta]^{T} } \\
\bar{x}_{R}= & \text { state vector containing states of the main rotor } \\
& \text { (and higher order dynamics not included in } \bar{x}_{B} \text { ) }
\end{aligned}
$$

The matrices $A_{B}$ and $B_{B}$ contain body-only dynamics $\mathrm{Ad}$ matrices $A_{R}$ and $B_{R}$ represent rotor-only dynamics, while the other matrices in (3.9) represent the coupling between the two state vectors. Model (3.9) can be reduced to the quasi-static case by letting $\dot{\bar{x}}_{R}$ equal zero and solving for $\overline{\boldsymbol{x}}_{\boldsymbol{R}}$ :

$$
\bar{x}_{R}=-A_{R}^{-1} A_{R B} \bar{x}_{B}-A_{R}^{-1} B_{R} \bar{u}
$$

Substitution of $\vec{x}_{R}$ in the body equations yields the quasi-static model:

$$
\dot{\bar{x}}_{B}=F \bar{x}_{B}+G \bar{u}
$$

where,

$$
\begin{aligned}
& F=A_{B}-A_{B R} A_{R}^{+1} A_{R B} \\
& G=B_{B}-A_{Q R} A_{R}^{1} B_{R}
\end{aligned}
$$

\subsection{Summary}

A general discussion of the integration, linearicatior, and dynamic stabilization methods used for the computer simulation was presented. It was shown that a dynamic accuracy of less than $0.1 \%$ requires an integration time step of less than 0.004 seconds. 


\section{Chapter 4}

\section{Model Validation}

To verify that the model (RBM) satisfactorily represents the true characteristics of the Bell 205, it is compared to flight test data (NRC) obtained from the NRC and also to the 6 D.O.F quasi-static model (QSM) presented in [3]. Comparisons are performed at hover, 40 knots, and 80 knots forward velocity using the linear perturbation version of the RBM model about the appropriate trim point ${ }^{1}$. The goal of these comparisons is to determine if the RBM model performs as well, if not better than the QSM model presently being used at NRC. If the RBM model meets this requirement then the higher order dynamics present make it a better choice for feedback control design.

Due to the unstable nature of the helicopter, the usual time domain identification techniques such as step or $p u$ ulse responses are not used. Such techniques can cause extreme attitude excursions from trim, especially at hover, thus making comparisons to linear perturbation models meaningless. In acilition, these techniques require precisely shaped inputs which often result in the use of control fixtures or digital controllers to assist the pilot. The frequency-domain technique used does not suffer from these drawbacks and it is the most readily available form of flight test data at the NRC.

\subsection{Frequency Domain Method}

The systern is excited with a swept sine wave (frequency sweep) containing a frequency range of approximately 0.3 to $15 \mathrm{rad} / \mathrm{s}$. This range of frequencies ensures the excitation of the rigid body modes as well as the structural modes which are dominant at about 14

\footnotetext{
'Linear model parameters are given in Appendix $E$ and $F$
} 


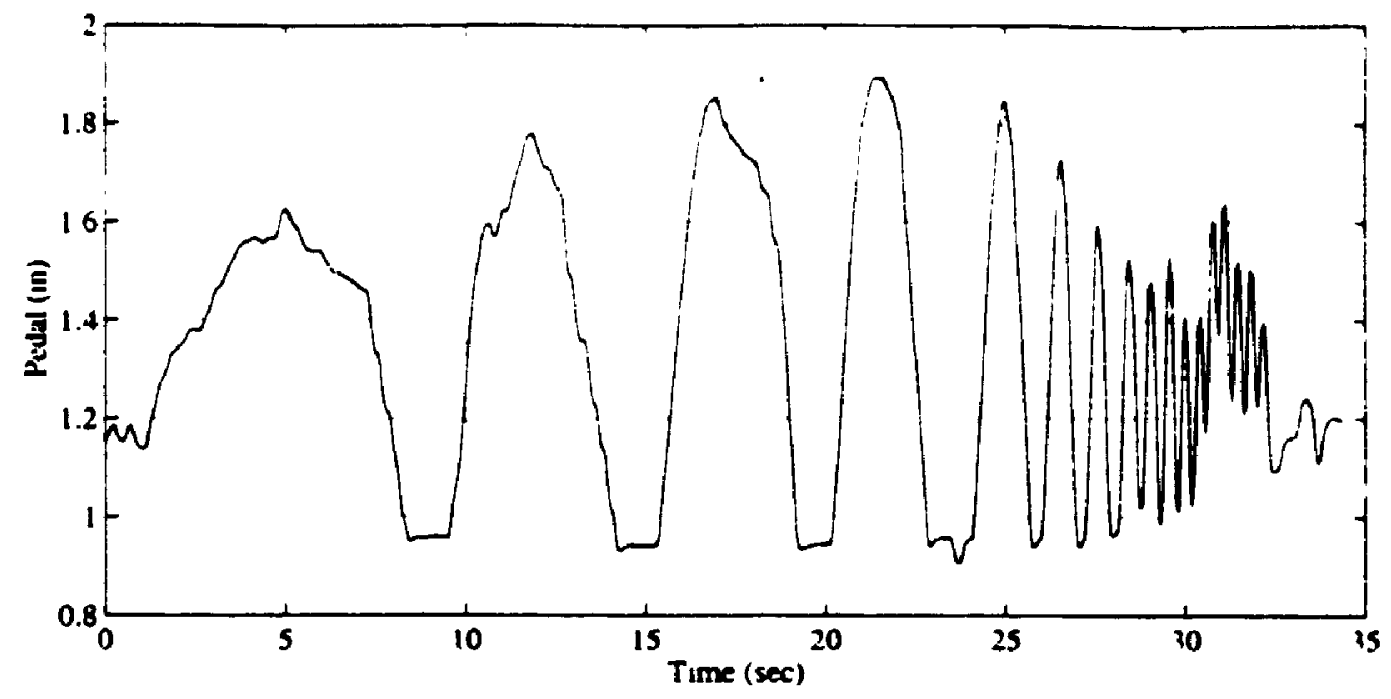

Figure 4.1: Typical frequency sweep pattern

$\mathrm{rad} / \mathrm{s}$.

A frequency sweep is performed by starting from trim and moving the control in a sinusoidal fashion starting with low frequency excursions and progressing to high frequency. The magnitude of the input sinusoid is varied in an attempt to keep the states near trim as can be seen from the sweep shown in figure 4.1. For each trim condition, frequency sweeps of the four input channels (main rotor collective (collective), lateral and longitudinal cyclic, and tail rotor collective (pedal)) are required. At least two frequency sweeps of each channel are desired to ensure a sufficient amount of data for analysis ${ }^{[39]}$. A sampling fiequency of $16 \mathrm{~Hz}$ was used in collecting the flight test data. This frequency being larger indn twice the highest frequency of interest helps avoid frequency aliasing errors which can occur in the sampling process.

\subsubsection{Data Analysis}

The spectral analysis techniques described in [40-43] were employed to calculate the gain an phase relationship between the input-output pairs. Basically, spectral analysis seeks to uncover the linear relationship between two sets of data in terms of a correlation function or its Fourier transiorm (spectral density function). From the measured input, $u(t)$, and output, $y(t)$, the estimated linear system frequency response function (or transfer function) 
may be calculated according to:

$$
H(f)=\frac{G_{u y}}{G_{u v}}
$$

where $G_{u u}$ is the one sided autospectral density function and $G_{u y}$ is the one sided crossspectral density function. Both $G_{u u}$ and $G_{u \nu}$ can be developed in terms of direct Fourier transformation of the original data samples ${ }^{[39]}$. A coherence iunction, $\gamma_{u y}^{2}(t)$, can also be defined between $u(t)$ and $y(t)$ which is a real valued quantity describing their linear relationship (i.e. the fraction of the output spectrum of $y(t)$ which is linearly due to $u(t)$ ):

$$
\begin{gathered}
\gamma_{u y}^{2}(t)=\frac{\left|G_{u y}\right|}{G_{u u} G_{y y}} \\
0 \leq \gamma_{u y}^{2}(t) \leq 1
\end{gathered}
$$

A value of 1 indicates direct linear relationship and a value of 0 indicates that the input and output are uncorrelated. With real data sets, the coherence function is usually less than one but greater than zero. A reduced correlation can be the result of one or more of the following:

1. The system is not linear and a linear relationship does not satisfactorily represent the system.

2. Other (off-axis) inputs besides $u(t)$ are affecting $y(t)$, either correlated to $u(t)$ or uncorrelated.

3. Non-random measurement noise, the random part being eliminated by the transfer function calculation.

4. The spectral estimates contain resolution bias errors.

The two mor importaut factors affecting the coherence function for a helicopter are nonlinearity and off-axis inputs. First, the helicopter possesses nonlinear qualities and second, the dynamics are highly coupled, resulting in extraneuus on-axis motion due to off-axis control inputs which are required to stabilize the helicopter while the frequency sweep is being performed. This is most evident at low frequencies. If this off-axis control is uncorrelated with the on-axis input then there will be a resulting drop in the coherence function. However, if the off-axis control is correlated with the input then the coherence function will not necessarily reflect the resulting error in the transfer function estimate. 
The off-axis control for a helicopter will usually be correlated to the on-axis control and therefore the pilot attempts to limit the off-axis control to the bare minimum required for safety purposes.

Attempts were made at applying the frequency identification method to the nonlinear RBM simulation model. Two problems could not be overcome: First, the input frequency sweep was altered by the controller, resulting in undesirable magnitude variation of the sweep, especially at low frequencies. Second, the amount of off-axis control exerted to scabilize the simulation during a frequency sweep resulted in poor coherence in the offaxis channels. Several attempts were made at controller design to limit the off-axis control inputs to the minimum required to insure stability without contaminating the on-axis response, however no success was found.

Matlab's signal processing toolbox ${ }^{[43]}$ was used to convert the time domain frequency sweeps of the NRC Bell 205 into standard Bode plots. The Matlab routines use Welch's method of power spectrum estimation along with data windowing and overlapping to improve the variance on the estimates. The window type selected was a Hanning window with overlap rates of approximately half the section length ${ }^{2}$. The data sets are first detrended to remove linear drifts and mean values, partitioned into overlapping sections, and then windowed prior to Fourier transform calculation.

The bode plot of the longitudinal cyclic to pitch rate of the Bell 205 at hover, figure 4.2, shows a high coherence function in the frequency range of 1 to $17 \mathrm{rad} / \mathrm{s}$ with a gradual roll off bellow this range and drastic roll off above. The high frequency coherence difficulty is due $x$, the lack of excitation whereas the drop in coherence at low frequency is due to secondary inputs and nonlinearities. The remaining bode plots for the NRC Bell 205 are presented in the next section and the corresponding time series data are presented in Appendix B. For clarity, the Bode plots of the flight test data are presented as continuous curves rather than the discrete points in which they were derived.

\footnotetext{
${ }^{2}$ this has been found to lower the variance on the eatimates sigaificantly $(43)$
} 

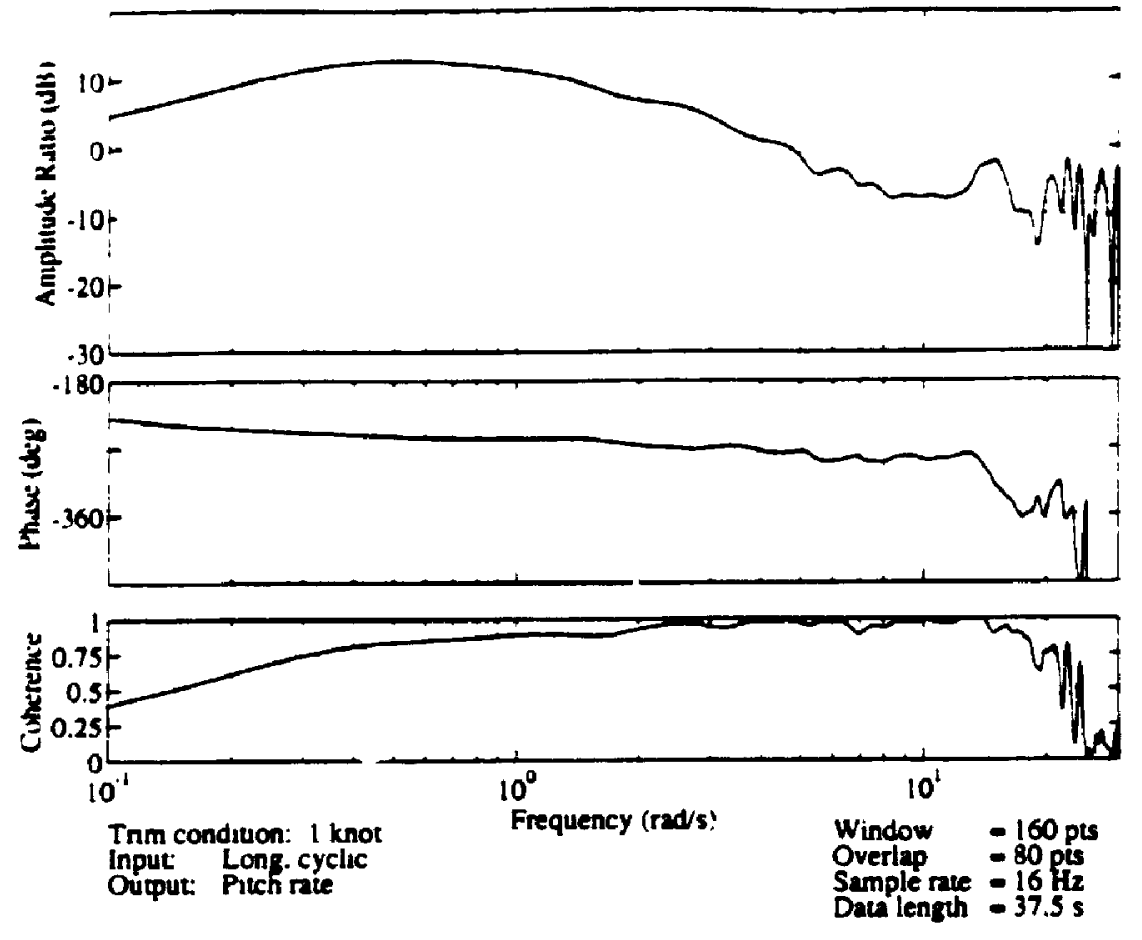

Figure 4.2: NRC Bell 205 longitudinal cyclic to pitch rate response at hover

\subsection{Model Comparison}

Prior to comparing the model to the flight test data and to the quasi-static model, the following control se :ivities $^{[3]}$ were applied to convert all inputs to the same units.

$\begin{aligned} \text { Collective: } & 2.446723 \times 10^{-2} \frac{\mathrm{rad}}{\mathrm{in}} \\ \text { ong. cyclic: } & 3.222146 \times 10^{-2} \frac{\mathrm{rad}}{\mathrm{in}} \\ \text { Lat. cyclic: } & 2.685122 \times 10^{-2} \frac{\mathrm{rad}}{\mathrm{in}} \\ \text { Pedal: } & 7.867407 \times 10^{-2} \frac{\mathrm{rad}}{\mathrm{in}}\end{aligned}$

In addition, the positive direction of the longitudinal and lateral cyclic inputs of the RBM model differ frotn those of the QSM and NRC. It was therefore necessary to shift the phase of the cortesponding RBM plots by $\pm 180^{\circ}$ before direct comparisons could be made. This 


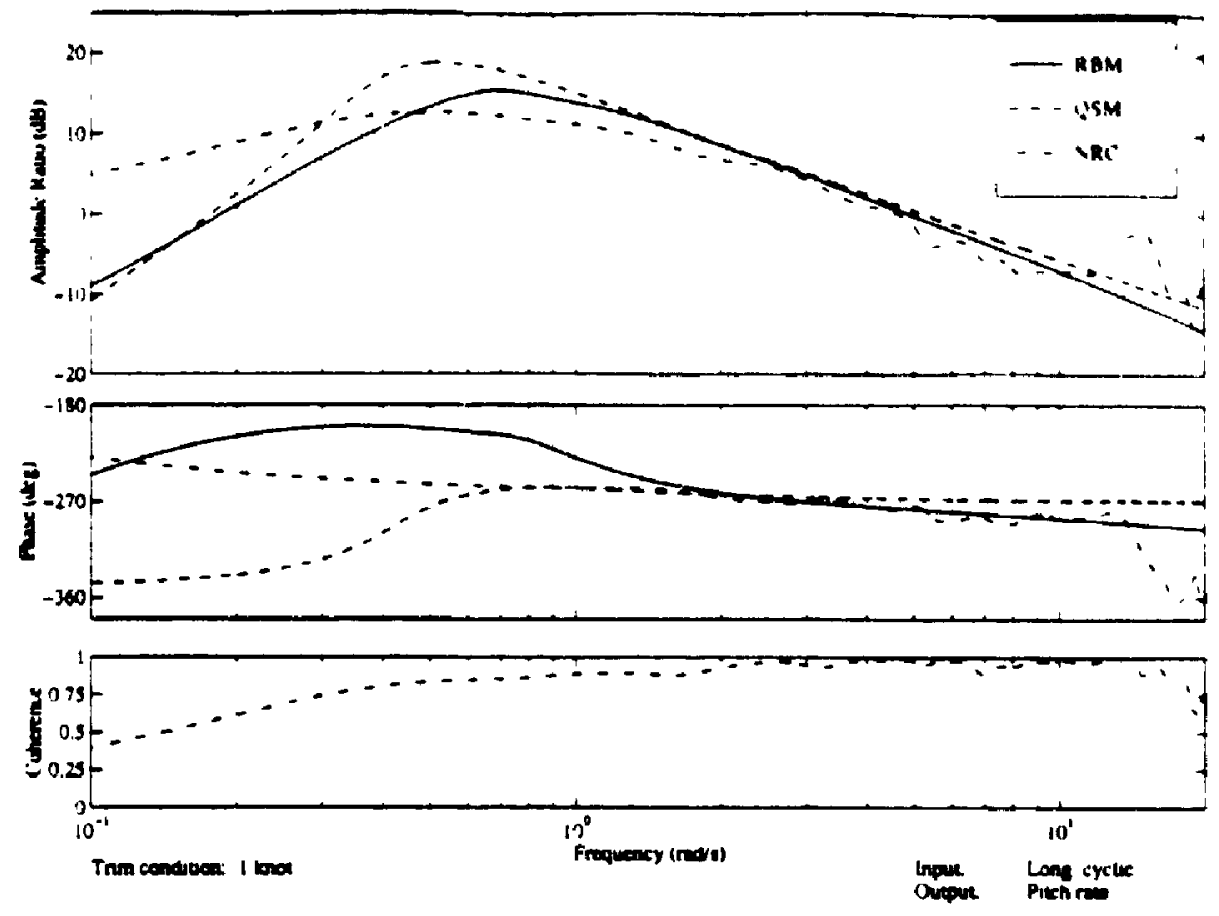

Figure 4.3: Longitudinal cyclic to pitch rate at hover

was accomplished by a change to the $B$ matrix of the RBM model:

$$
B_{R B M} \Rightarrow B_{R B M}\left[\begin{array}{cccc}
1 & 0 & 0 & 0 \\
0 & -1 & 0 & 0 \\
0 & 0 & -1 & 0 \\
0 & 0 & 0 & 1
\end{array}\right]
$$

The frequency domain comparisons are presented in figures 4.3 through 4.45. Each plot contains the magnitude and $\mathrm{p}^{\prime}$ sse of each model and flight test data. The coherence funstion is also plotted to gaug the linearity and/or reliability of the flight test data ${ }^{3}$. The hover trim condition presented is actually for a forward speed of 1 knot which was chosen to correspond to the trim condition of the QSM hover model.

\subsubsection{On-Axis Response}

The longitudinal cyclic to pitch rate responses are plotted in figures 4.3 through 4.6. At hover, figure 4.3 , both models agree well with the flight test data in the frequency range of 0.7 to $11 \mathrm{rad} / \mathrm{s}$ above which au unmodeled resonant peak occurs due to the dynamic nature

${ }^{3}$ The coherence function for the RBM and QSM models has a value of 1.0 for all frequencies 

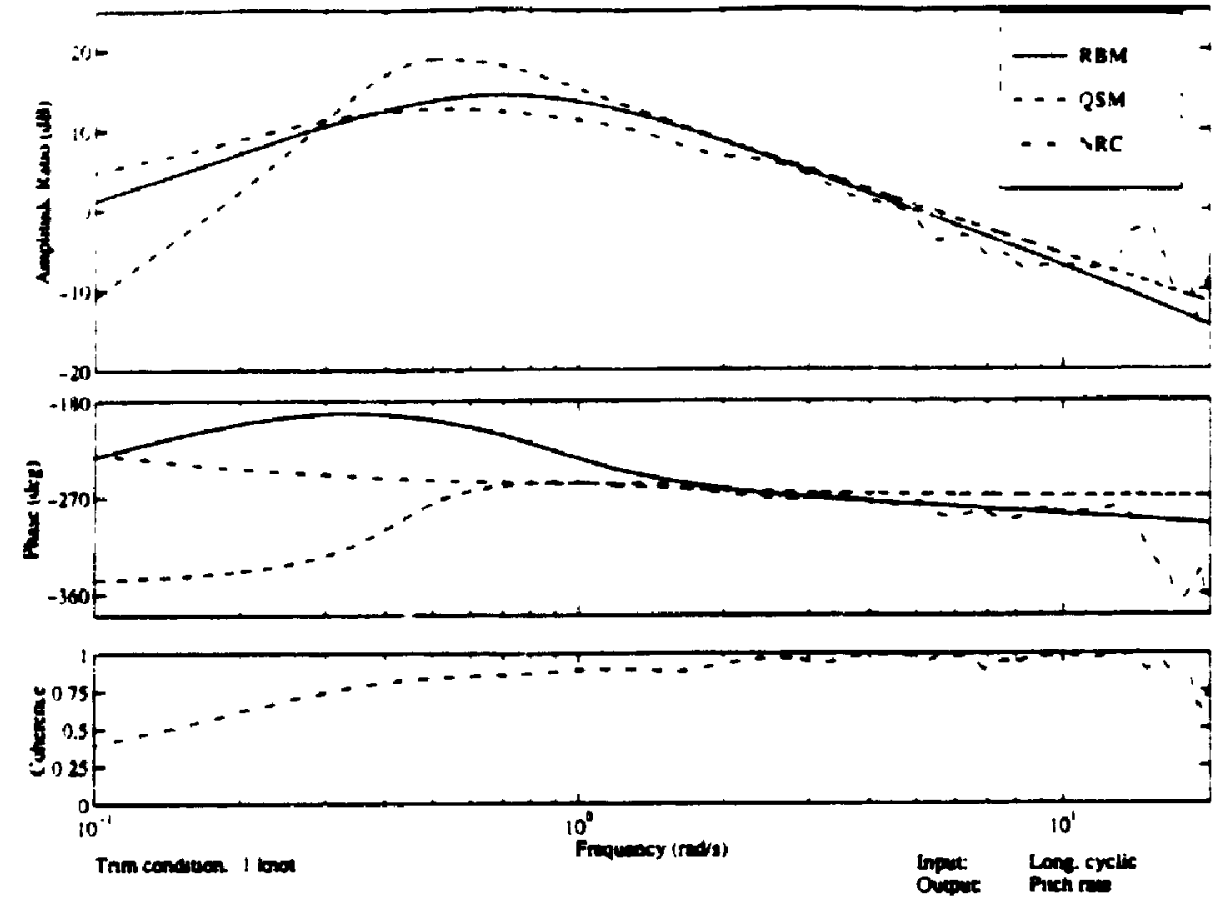

Figure 4.4: Longitudinal cyclic to pitch rate at hover $\left(\Delta v_{r_{R}}=0.01 \mathrm{~m} / \mathrm{s}\right)$

of the rotor-fuselage connection (pylon mode). Bellow this range both models predict a magnitude which is rising at approximately $30 \mathrm{~dB}$ per decade compared to the $15 \mathrm{~dB} / \mathrm{dec}$ rise of the flight test data. The low frequency phase of the RBM model differs from both QSM and NRC. However, the RBM model has a general trend in the right direction as the low frequency phase should tend to $-180^{\circ}$, not $-360^{\circ}$. The QSM model has a better phase match in the frequency range 0.5 to $1.5 \mathrm{rad} / \mathrm{s}$. It is possible to improve the low frequency magnitude of the RBM model by reducing the tail rotor induced velocity perturbation, figure 4.4. However since the low frequency coherence is low in this region it is uncertain whether the corresponding flight test data represents the correct characteristics of the helicopter and in fact it was found that a change in the window length (and overlap rate) which causes a corresponding change in the slope of the coherence function will also change the low frequency slope of the magnitude and phase functions in the low frequency region.

The same general comments apply to the 40 knots trim condition, figure 4.5 , and the 80 knots trim condition, figure 4.6. In bo:h cases the RBM model has a better high frequency phase prediction than the QSM model, whereas in the mid frequency range the opposite is true. The low frequency phase of the RBM model is seen to deteriorate with 


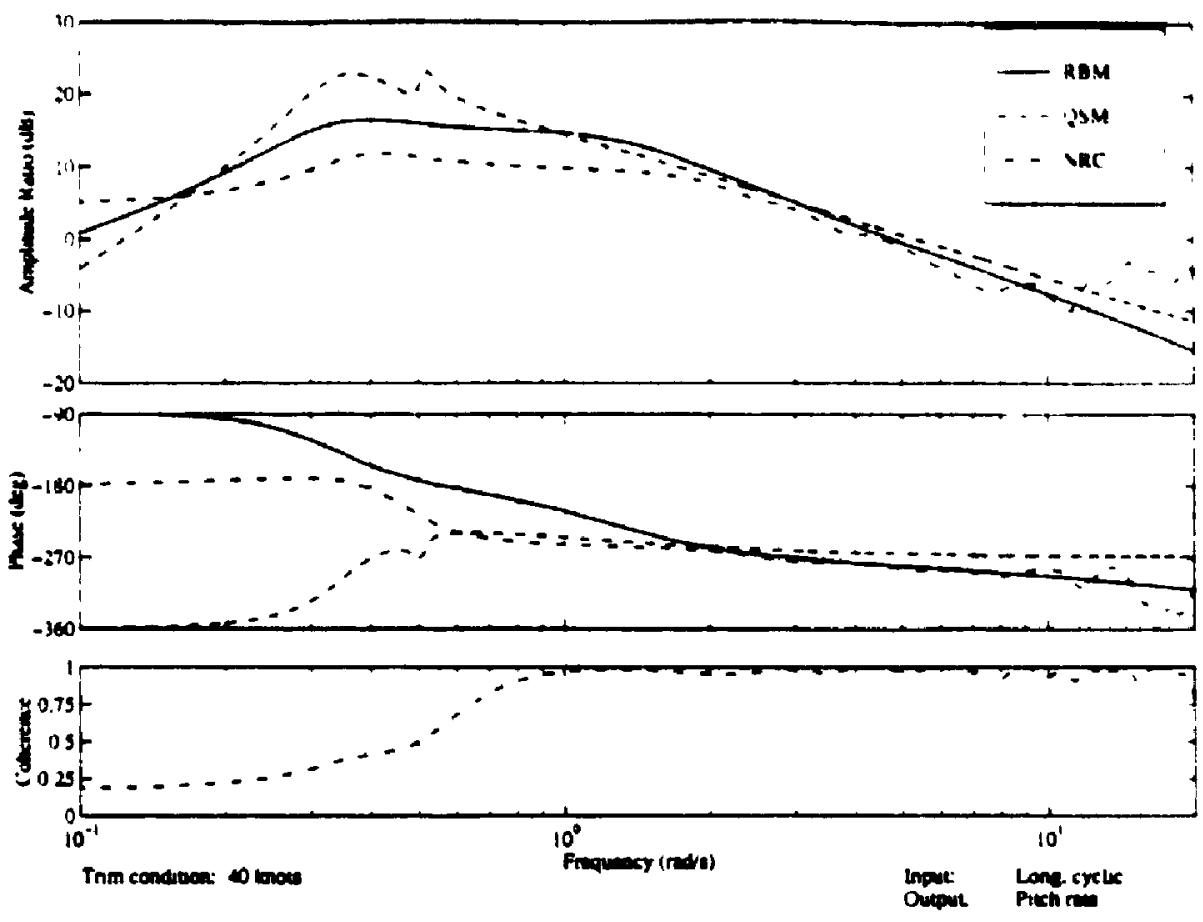

Figure 4.5: Longitudinal cyclic to pitch rate at 40 knots
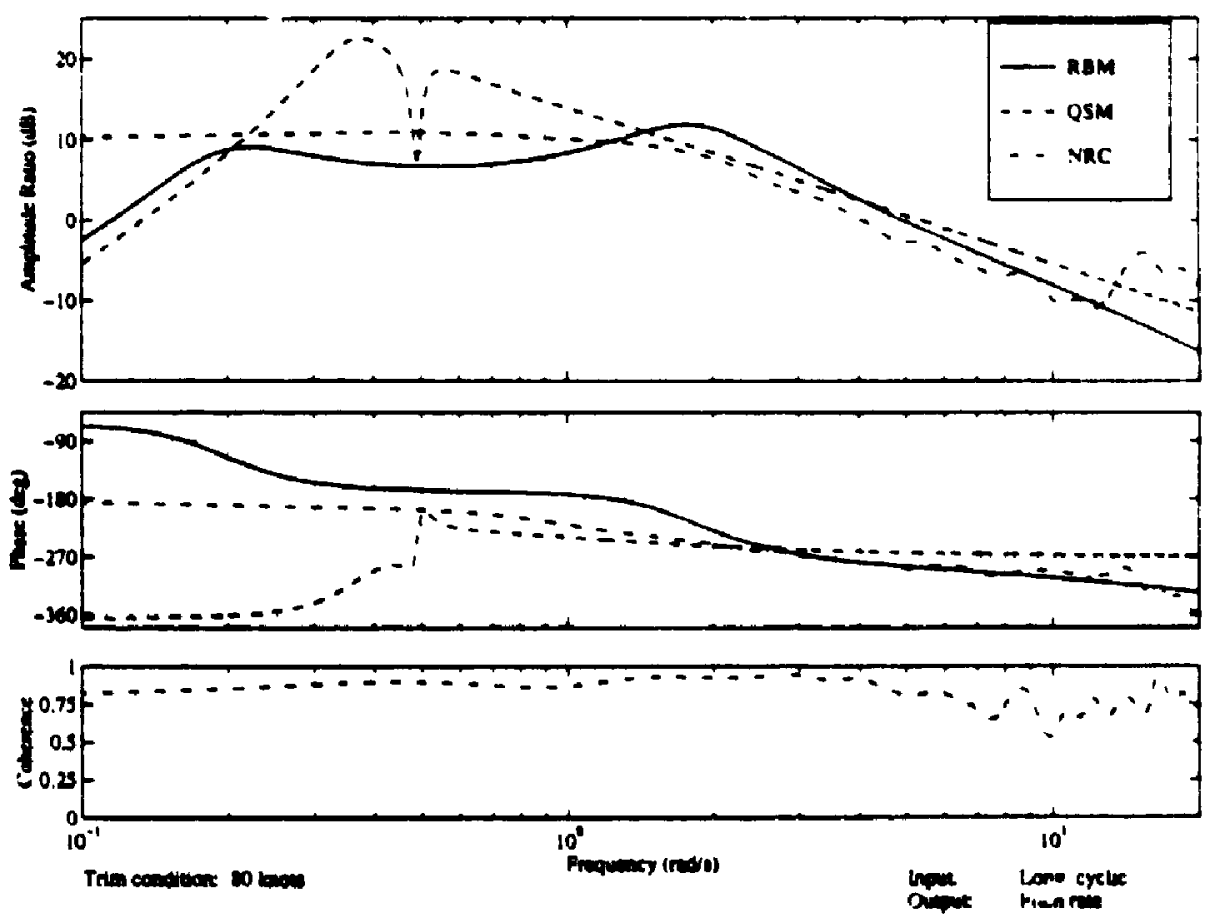

Figure 4.6: Longitudinal cyclic to pitch rate at 80 knots 


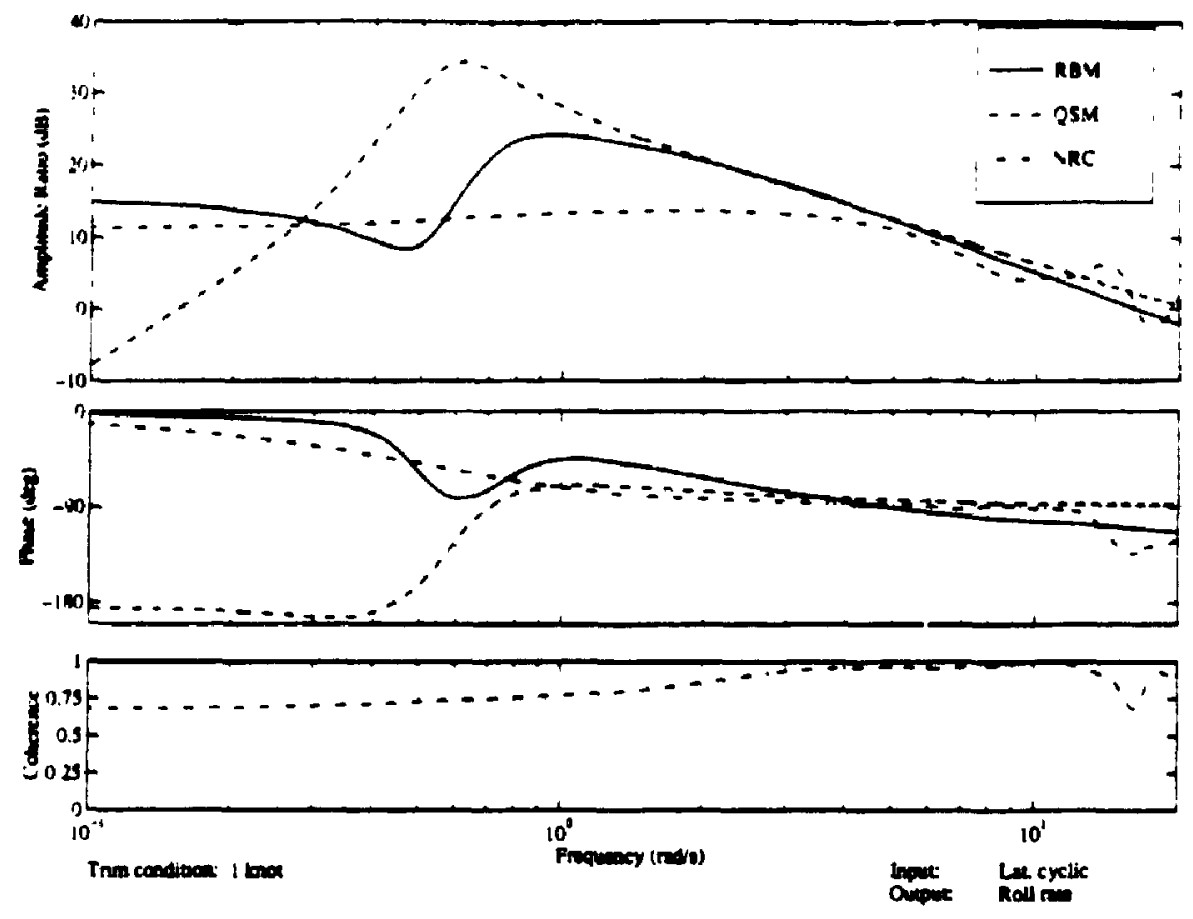

Figure 4.i: Lateral cyclic to roll rate at hover

forward velocity.

The lateral cyclic to roll rate response plots are shown in figures 4.7 through 4.9. The magnitude of the response is over predicted at all speeds with the greatest(approx. $12 \mathrm{~dB}$ ) deviations occurring at hover and in the mid frequency range. The magnitude prediction improves with forward velocity and is in general better than the QSM model. The low frequency phase prediction is an improvement over the QSM model, and also tends to improve with forward velocity. However, the slope of the phase curve in the mid to high frequency range is better predicted by the QSM model.

Pedal to yaw rate frequency response plots are presented in figures 4.10 to 4.12 . The best overall match is at hover, fig. 4.10 , where both models predict similar responses. The high frequency magnitude and phase of the flight test data are not entirely reliable due to the low and erratic coherence function, but it is apparent that both models predict a greater $(5 \mathrm{~dB})$ input to output sensitivity than the Bell 205. Even though there is some uncertainty about the actual value of the lift curve slope of the tail rotor bladest. the uncertainty can only account for $0.8 \mathrm{~dB}$ of the discrepancy found, leading to the

\footnotetext{
tvaluee ranging from 5.73 to 6.6 have been found
} 

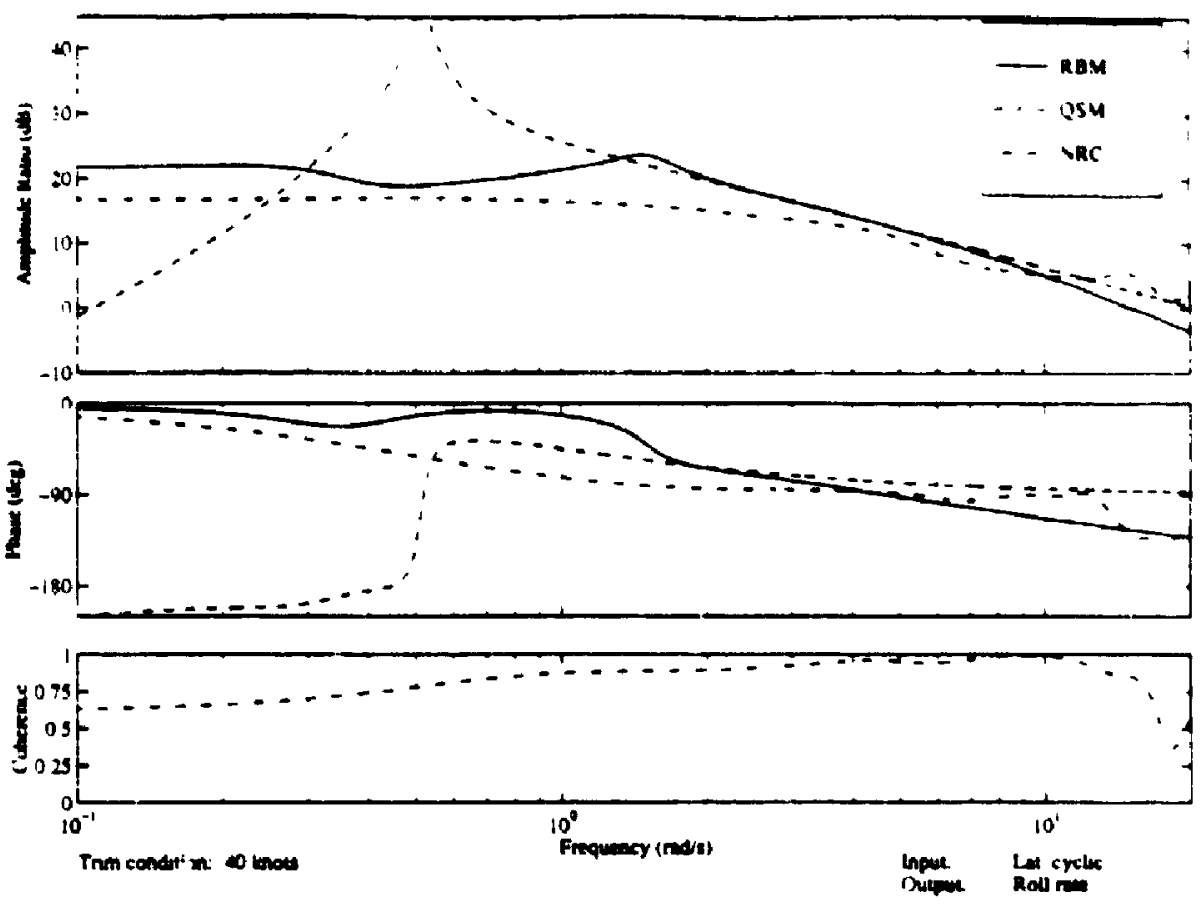

Figure 4.8: Lateral cyclic to roll rate at 40 knots
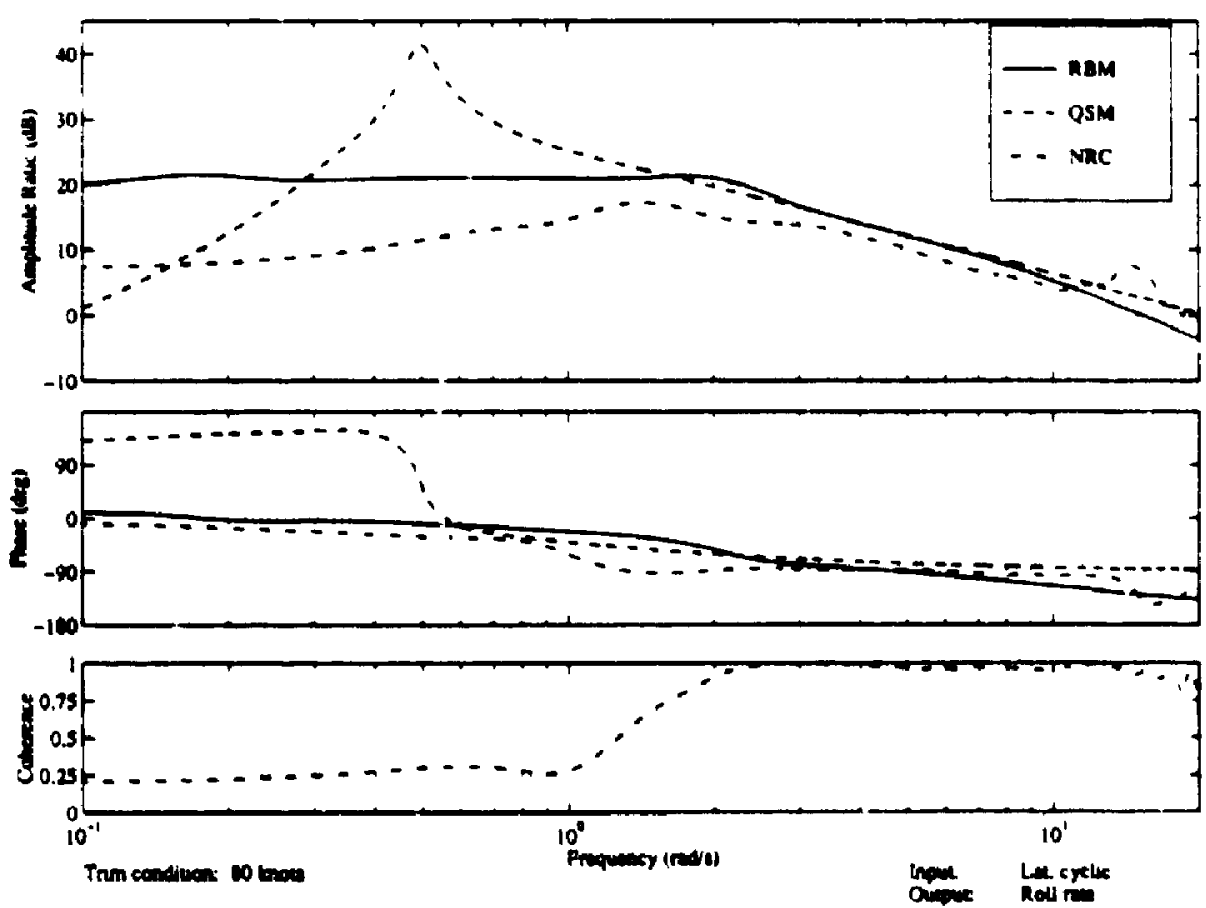

Figure 4.9: Lateral cyclic to roll rate at 80 knots 

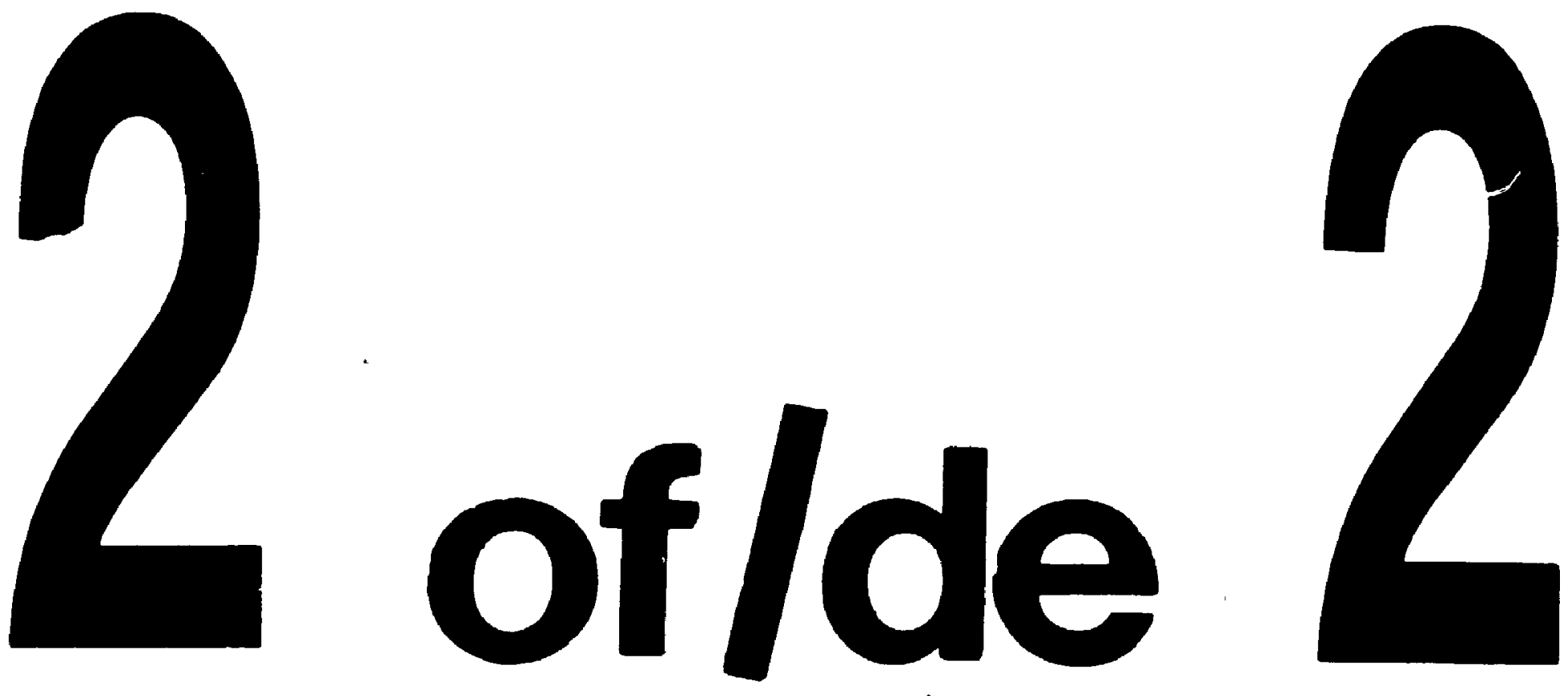

PM-1 31/2"x4" PHOTOGRAPHIC MICROCOPY TARGET NaS 1010a ANSI/ISO \#2 EQUIVALENT

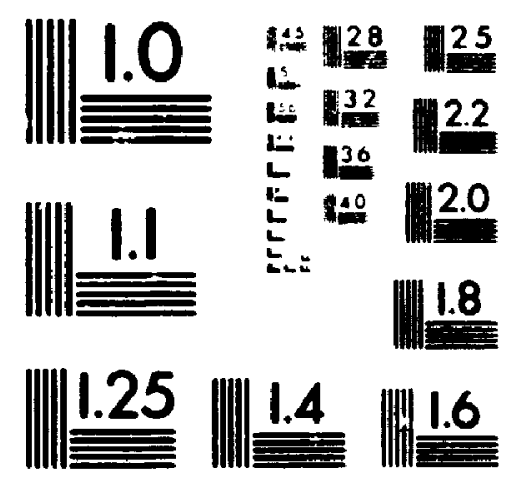

PRECISIONEM RESOLUTION TARGETS 

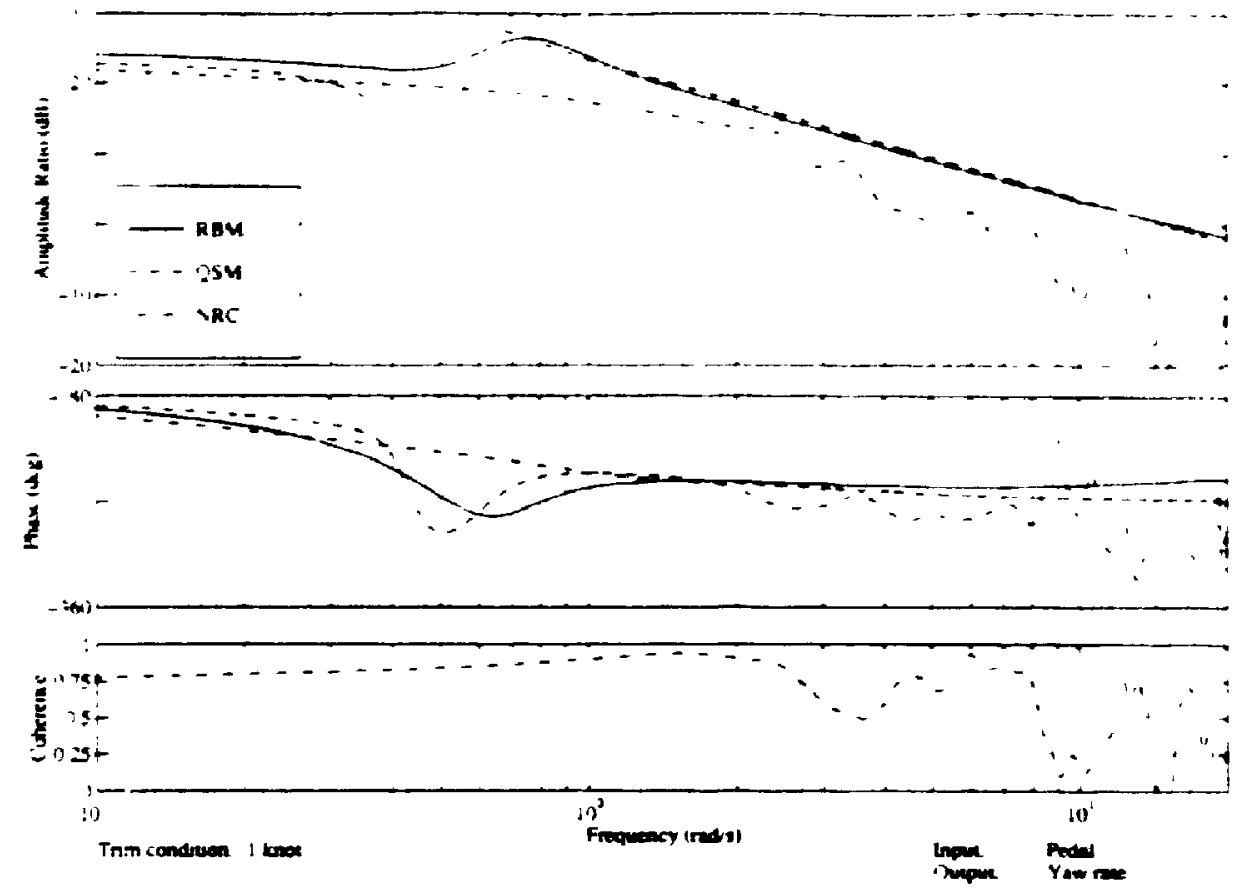

Figure 4.10: Pedal to yaw rate at hover

conclusion that an unmodeled phenomenon exists in the yaw axis. This phenomanon is believed to be the main rotor sidewash which is produced by the swirling motion of the main rotor wake at the tail rotor. The usual approach used in sidewash modeling at the tail rotor is to create a lookup table from either wind tunnel experiments or by using the flat-vortex wake model of the reference [44] as described in [2]. The sidewash offects were left unmodeled because wind tunnel data was not avai'.uble and accurate application of flat-vortex theory was beyond the scope of the present work.

At forward speeds, figures 4.11 and 4.12 , both models have two major slope rhanges which are not ronfirmed by the flight test data. The phase of the RBM model becomes unacceptable below a frequency of $1.1 \mathrm{rad} / \mathrm{s}$ at $40 \mathrm{knots}$ and approximately $2.2 \mathrm{rad} / \mathrm{s} \mathrm{at}$ 80 knots. The QSM model has superior phase characteristics below these frequencies. The nonlinear version of the QSM model ${ }^{[27]}$ incorporates the complete main rotor flow effects at the tail rotor and thus the stability derivatives of the linear QS.M model alst. includes these effects in a quasi-static fashion (resulting in a good low frequency match). Due to the increased sensitivity with tcrward speed, the RBM phase chararteristica ran be improved by choosing a smaller perturbation in body angles (figs. 4.13 and 4.14 ). Fivn 


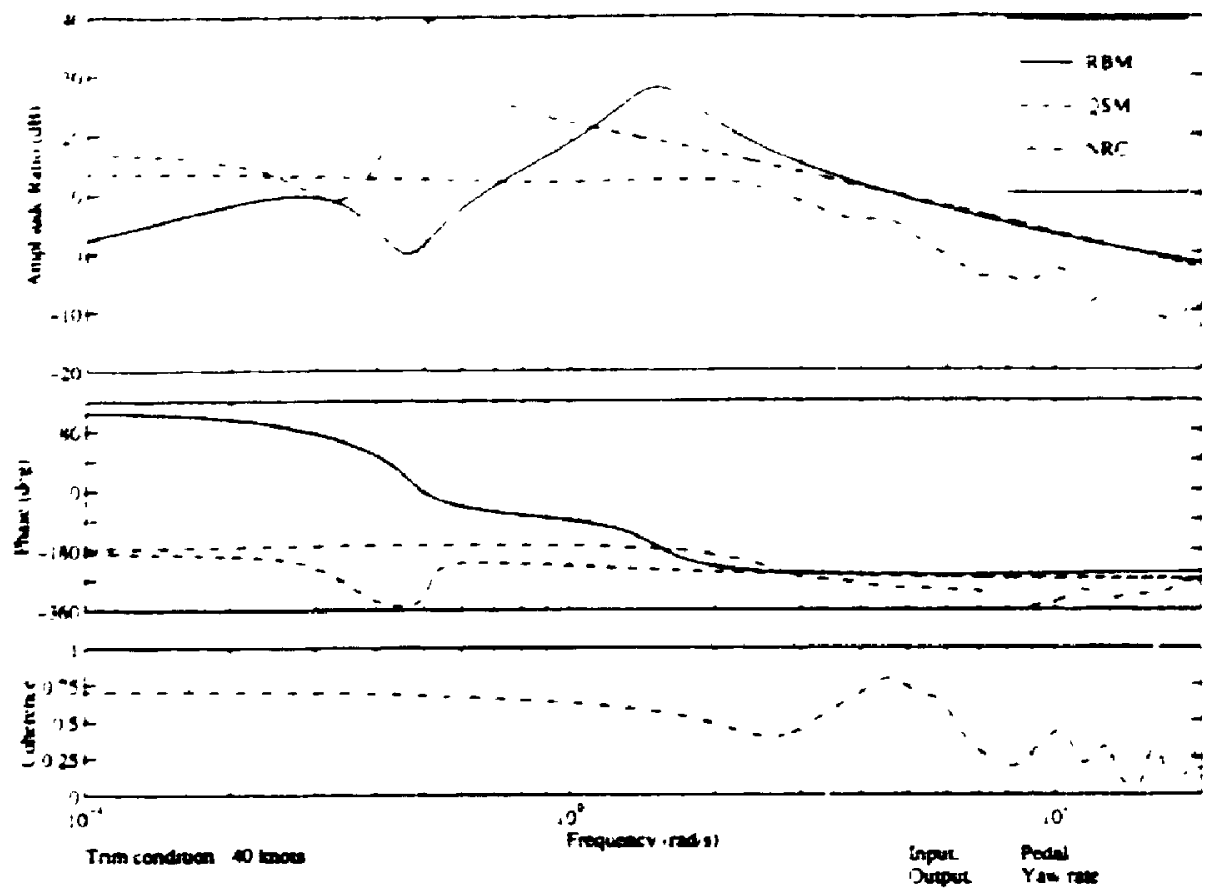

Figure 4.11: Pedal to yaw rate at 40 knots

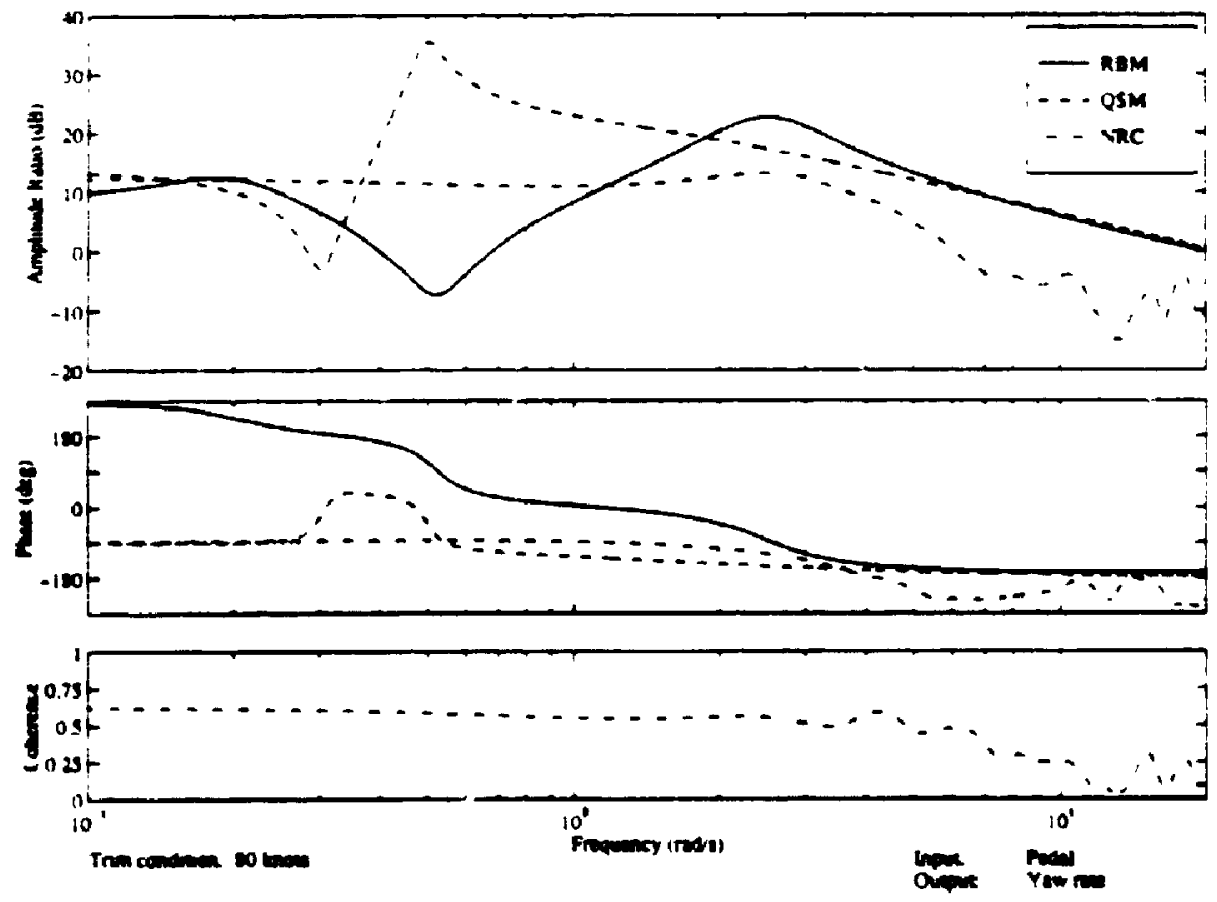

Figure 4.12: Pedal to yaw rate at 80 knots 

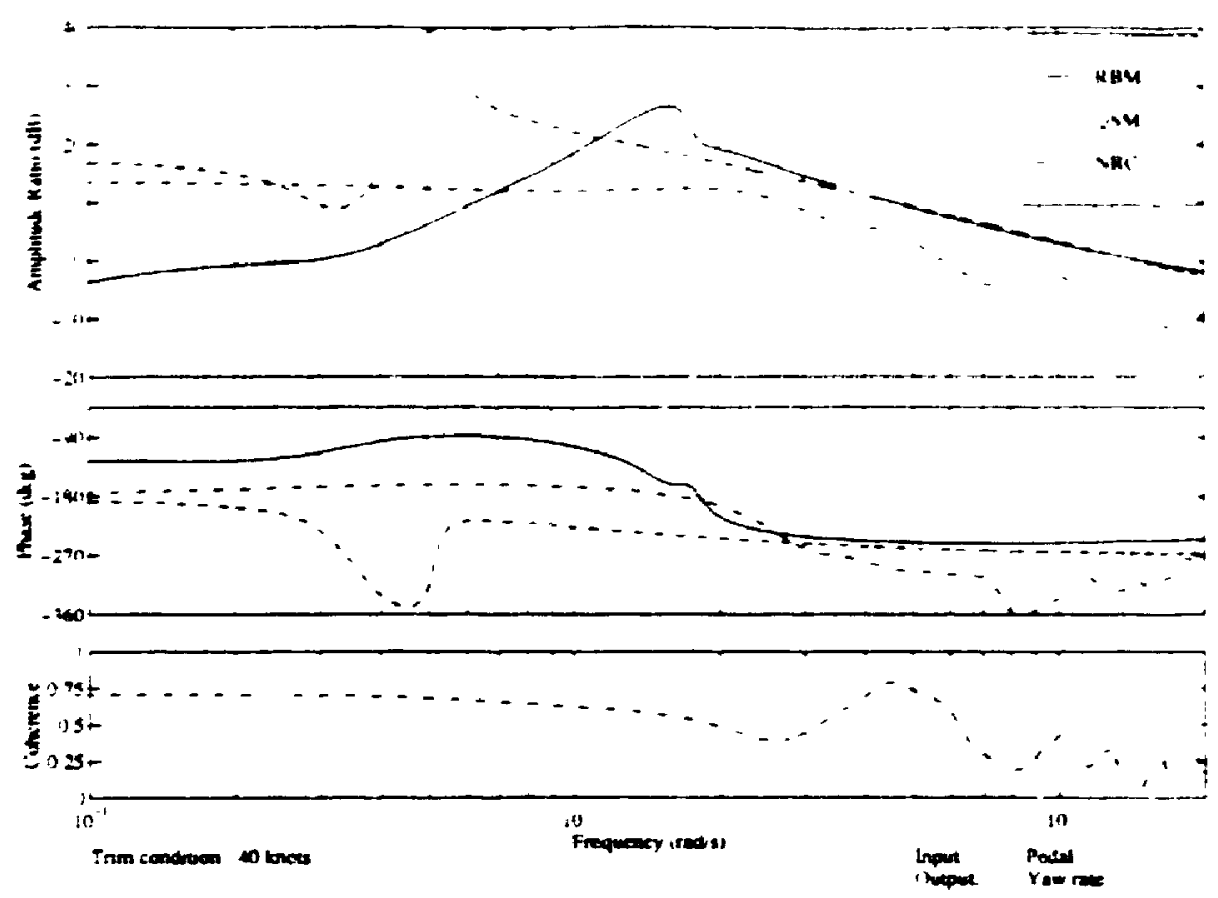

Figure 4.13: Pedal to yaw rate at 40 knots $(\Delta 0=0.01$ deg $)$

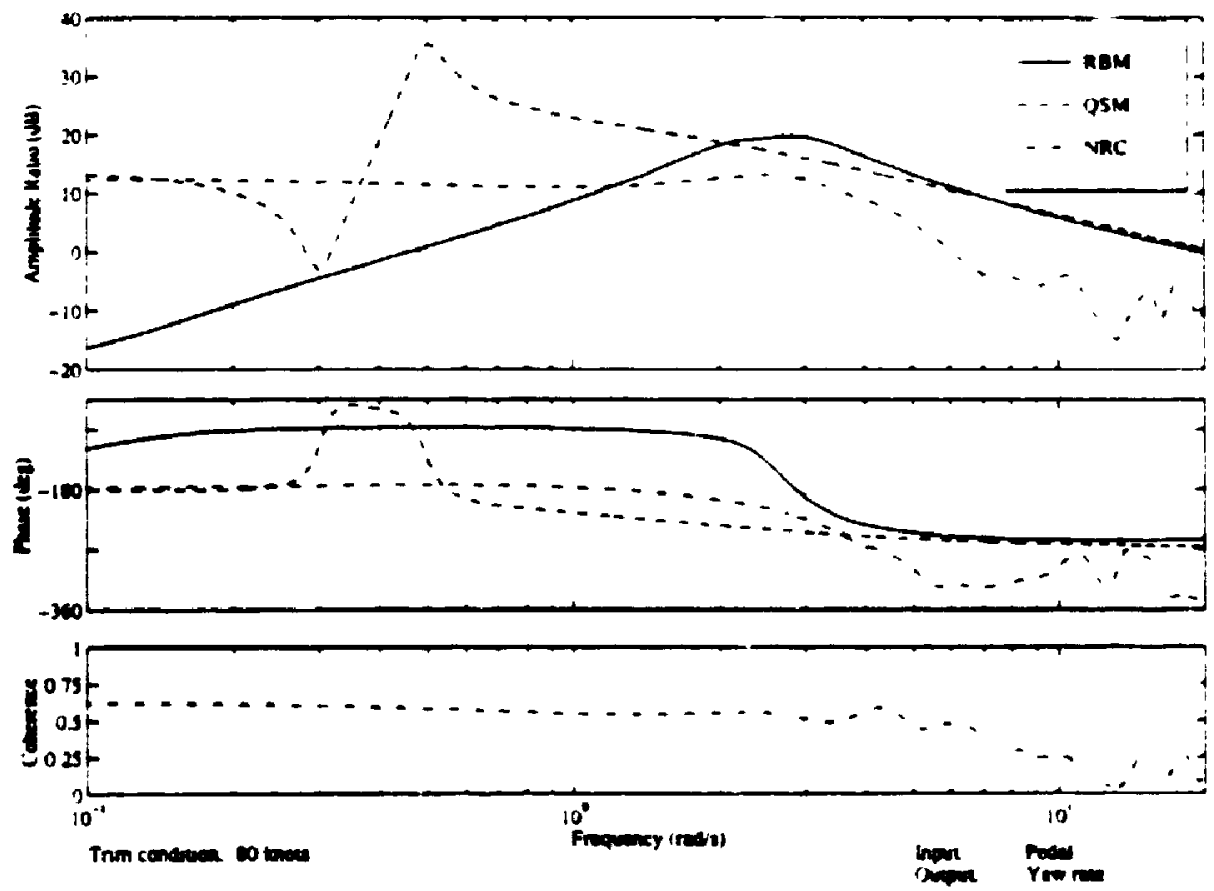

Figure 4.14: Pedal to yaw rate at 80 knota $(\Delta \bar{\phi}=0.01 \mathrm{deg})$ 


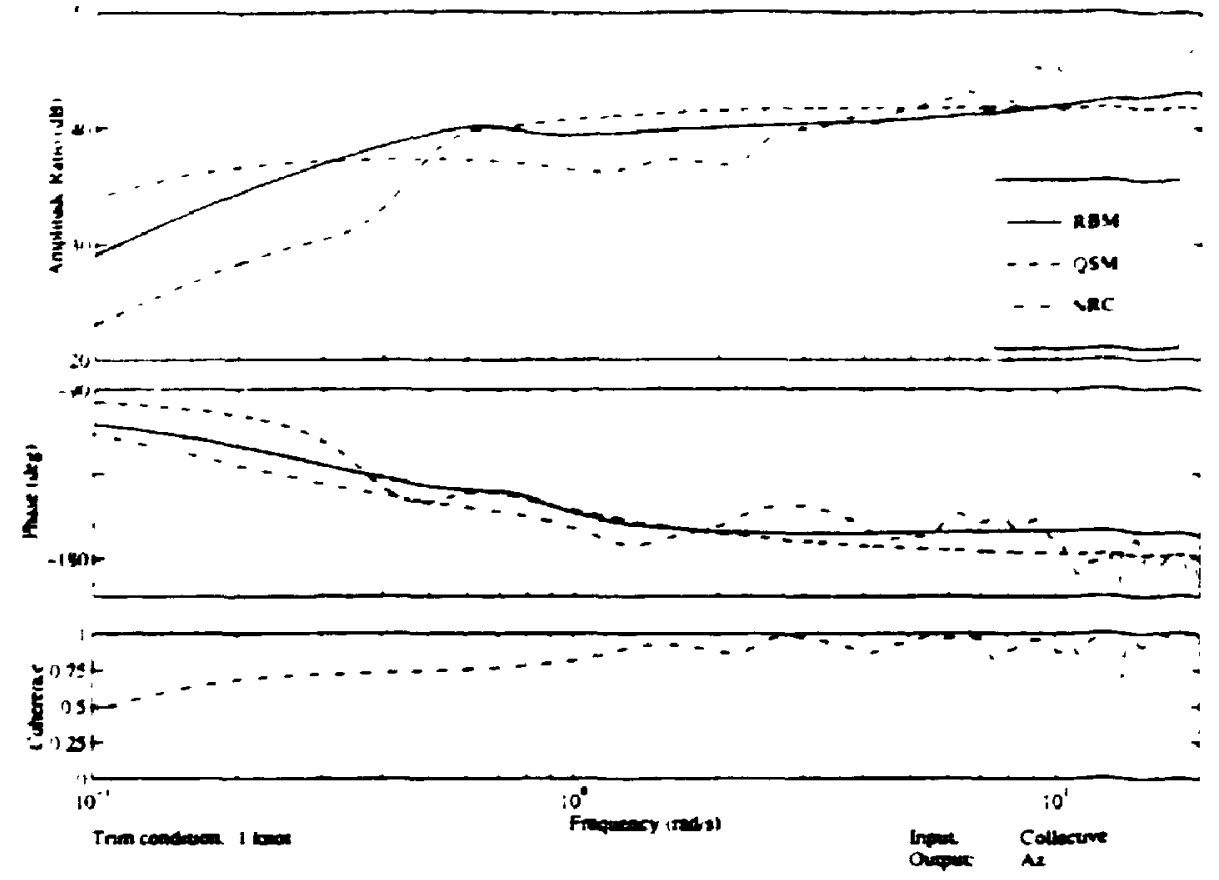

Figure 4.15: Collective to vertical acceleration at hover

though the model is somewhat improved. the cost is a deteriorated $f$, to $q$ and $A$ to $p$ response below $2 \mathrm{rad} / \mathrm{s}$.

Main rotor collective to vertical acceleration, $a_{z}$, frequency response plots are presented in figures 4.15 \% 4.17 . At hover, figure 4.15 , there is good agreement with both the flight test data and the QSM model. The low frequency magnitude and high frequency magnitude slopes are better predicted by the RBM model. At $40 \mathrm{knots}$, figure 4.16 . the match in phase is generally good whereas the magnitude prediction deteriorates below a frequency of $0.5 \mathrm{rad} / \mathrm{s}$. At 80 knots, figure 4.17 , a good match is found above $1 \mathrm{rad} / \mathrm{s}$ and below $0.2 \mathrm{rad} / \mathrm{s}$ where both models predict similar responses in phase $\left( \pm 360^{\circ}\right)$ and magnitude. In the faequency range 0.2 to $1 \mathrm{rad} / \mathrm{s}$ the RBM model has poor mag./phase characteristics which are in part due to the linearizing process; the model in forward flight is greatly improved by choosing a smaller body angle perturbation (figures 4.18 and 4.19 ).

The longitudinal cyclic to forward acceleration responses are portrayed in figures 4.20 to 4.22 . Good model prediction is found only in the high frequency range. $2.5 \mathrm{rad} / \mathrm{s}$ to $1.2 \mathrm{rad} / \mathrm{s}$. The RBMI and QSM models predict similar responses at hover and 40 knots. 


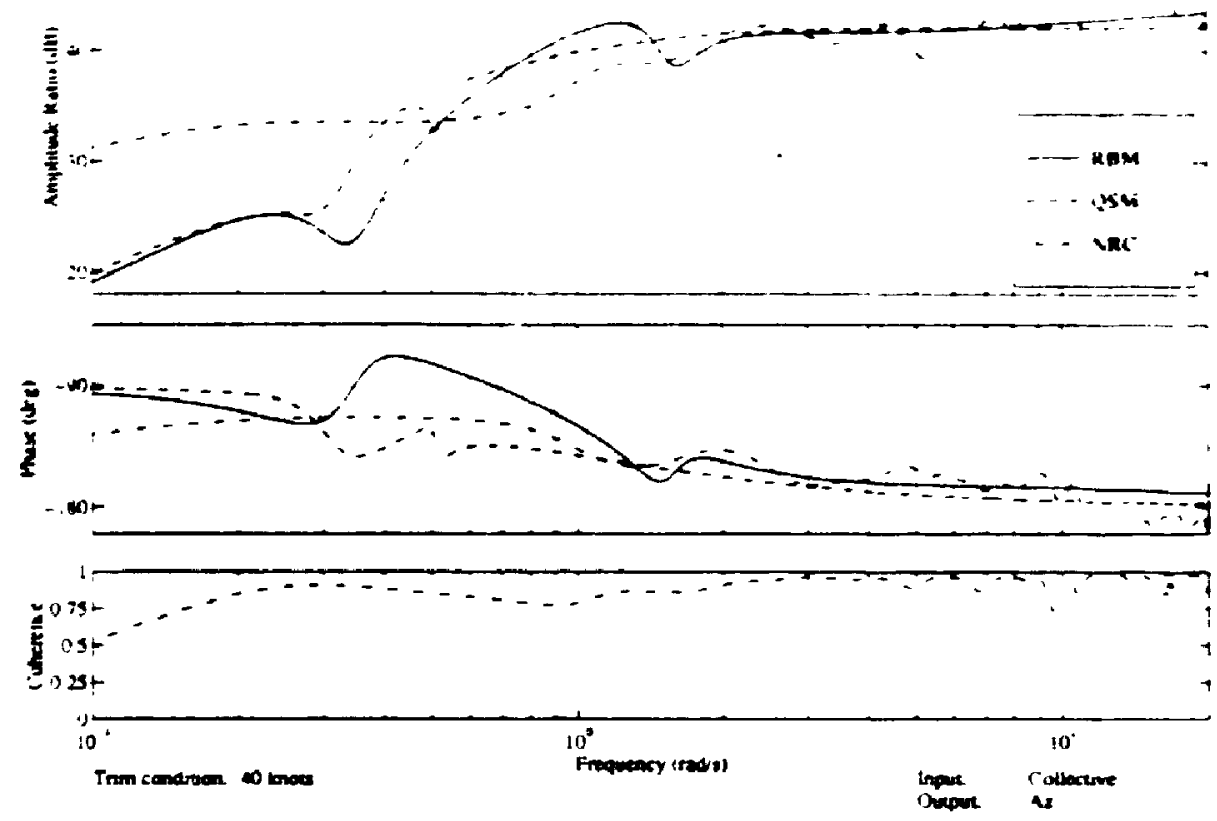

Figure 4.16: Collective to vertical acceleration at 40 knots

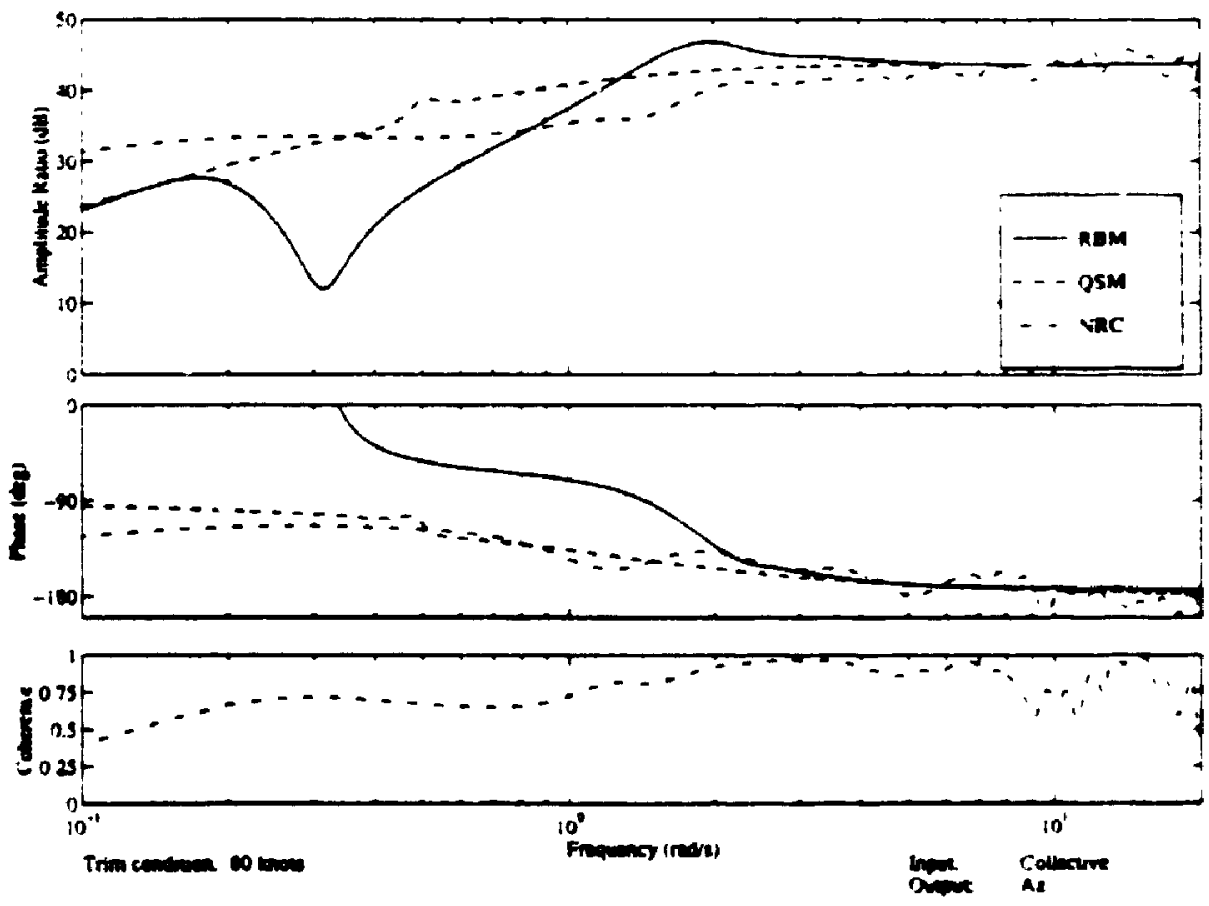

Figure 4.17: Collective to vertical acceleration at 80 knots 


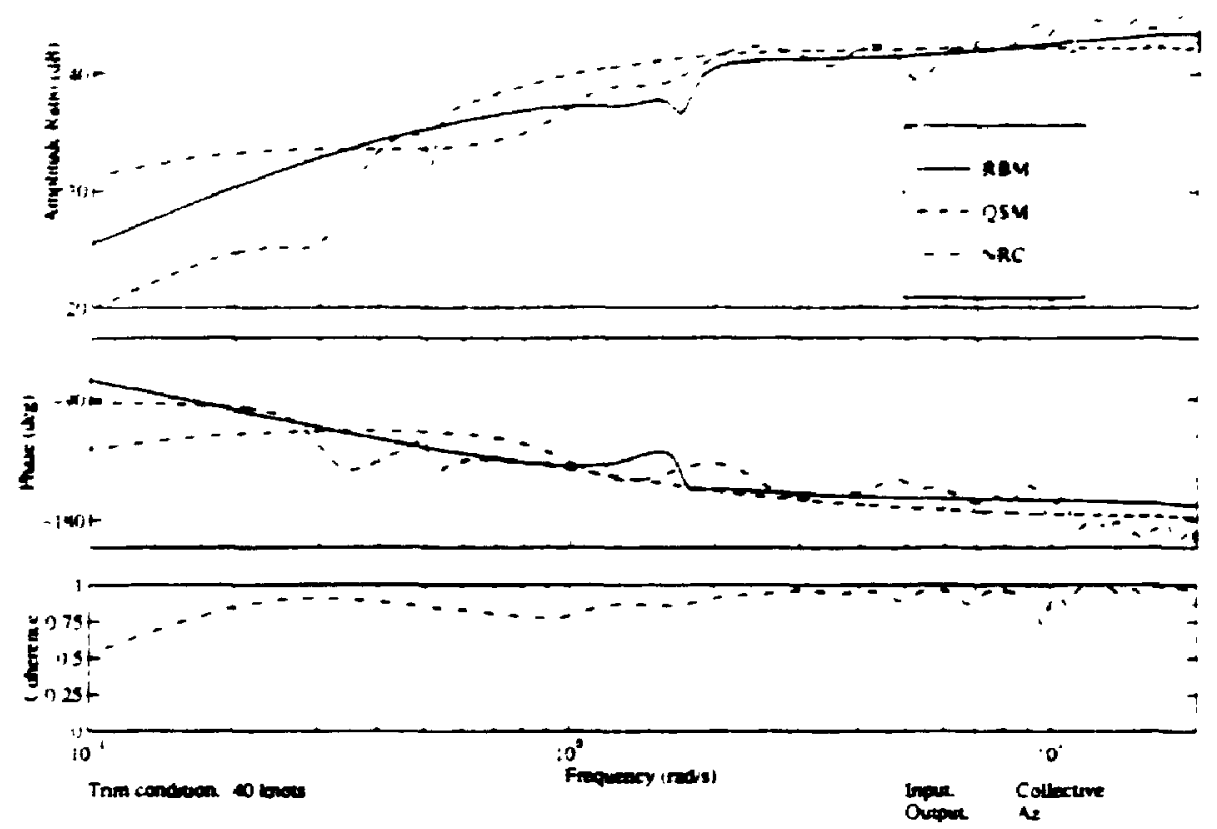

Figure 4.18: Collective to vertical acceleration at $40 \mathrm{knots}(30=0.01 \mathrm{deg})$

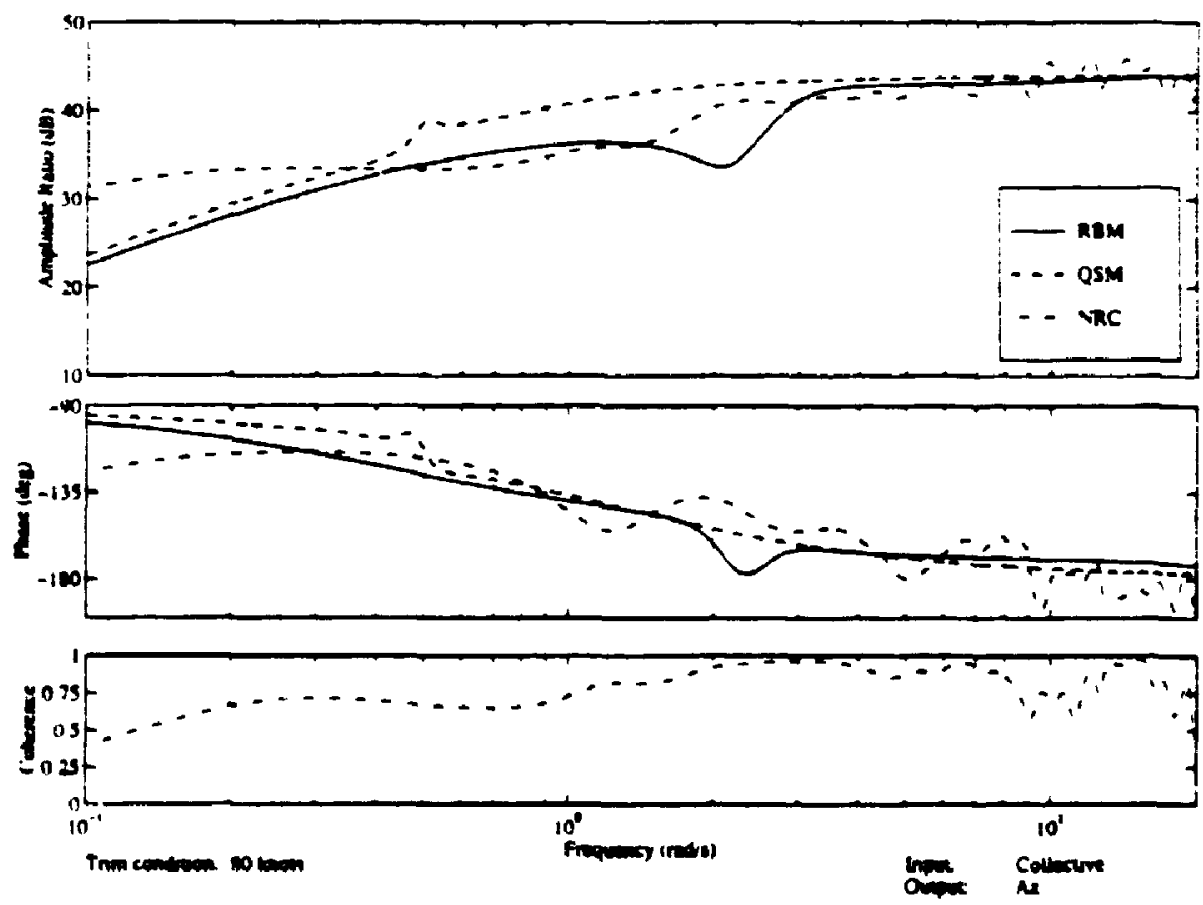

Figure 4.19: Collective to vertical acceleration at 80 knots $(\Delta \bar{\phi}=0.01 \mathrm{deg})$ 
'HA PIER 4. .WODEL IILIDATION

41

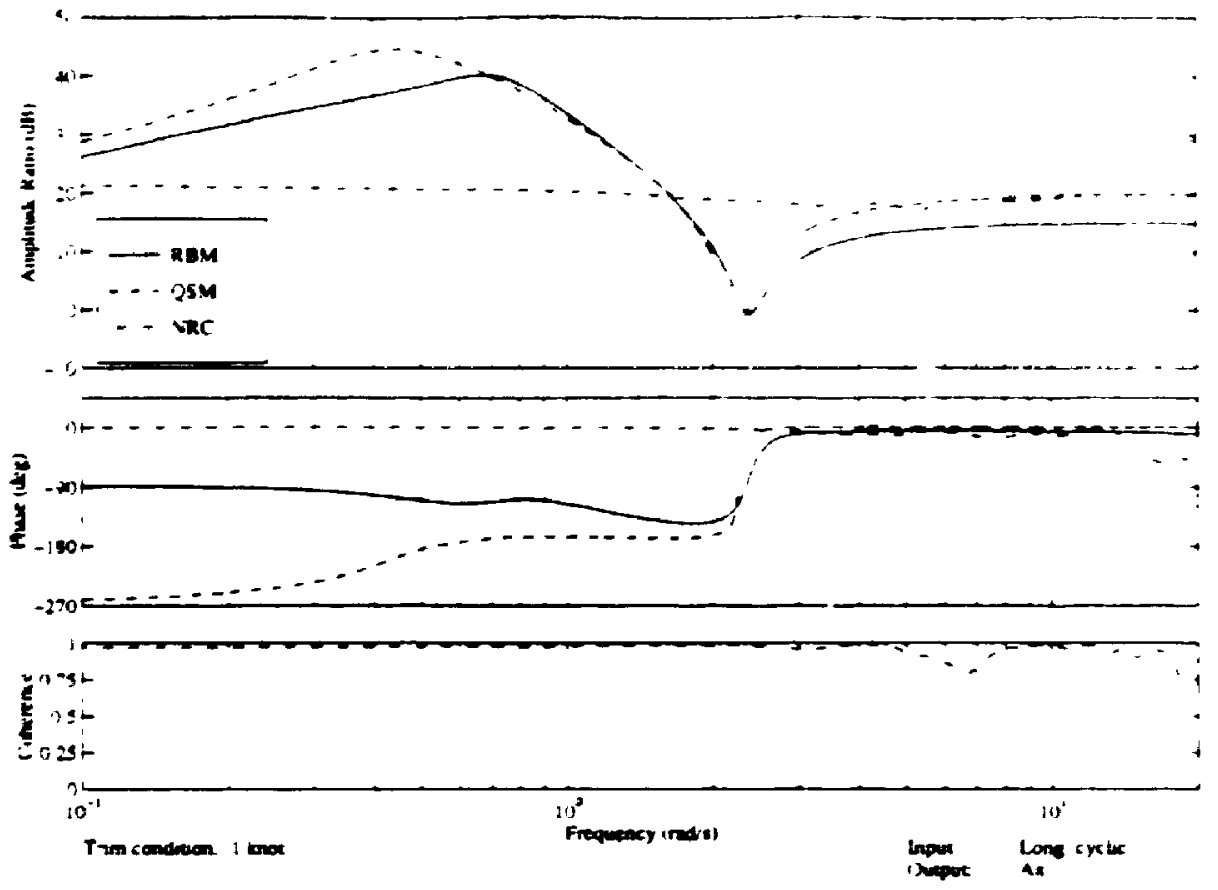

Figure 4.20: Longitudinal cyclic to forward acceleration at hover
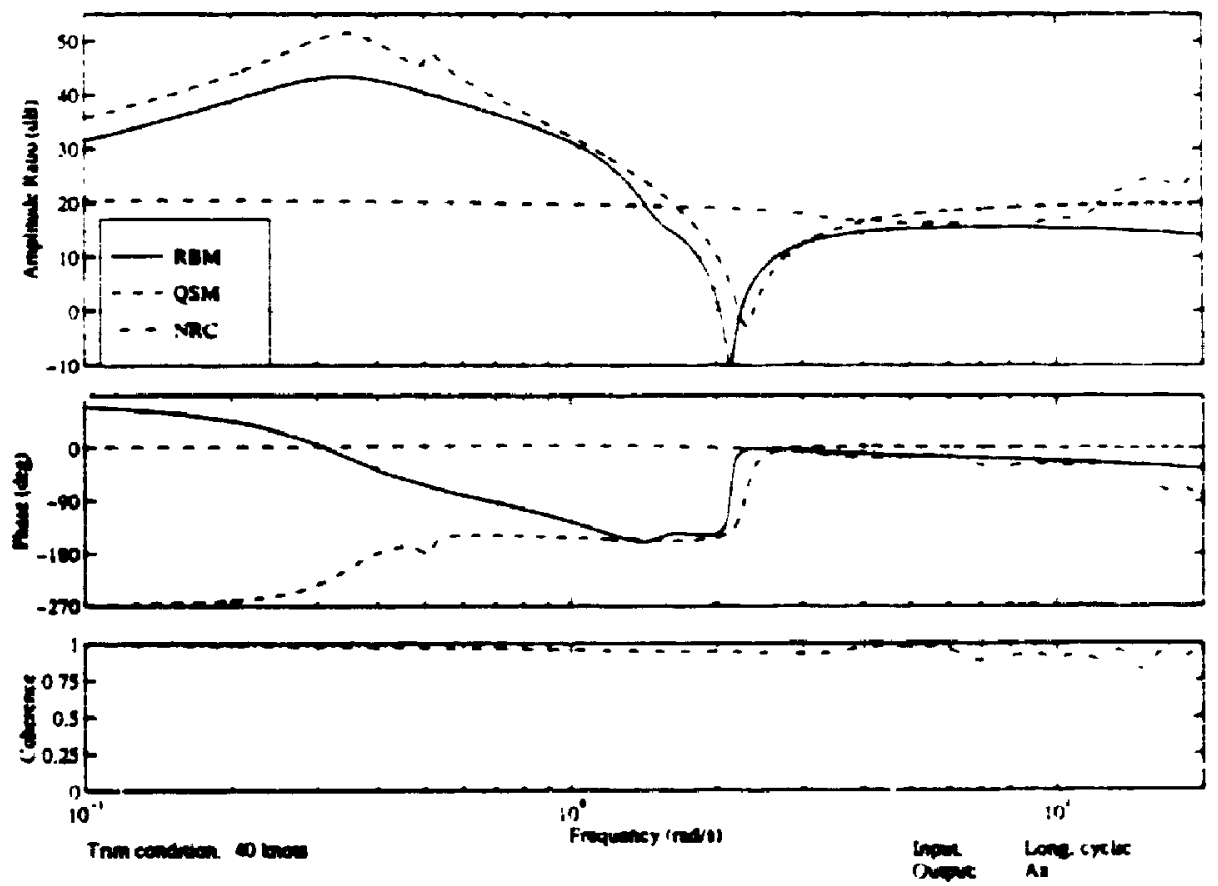

Figure 4.21: Longitudinal cyclic to forward acceleration at $40 \mathrm{knots}$ 


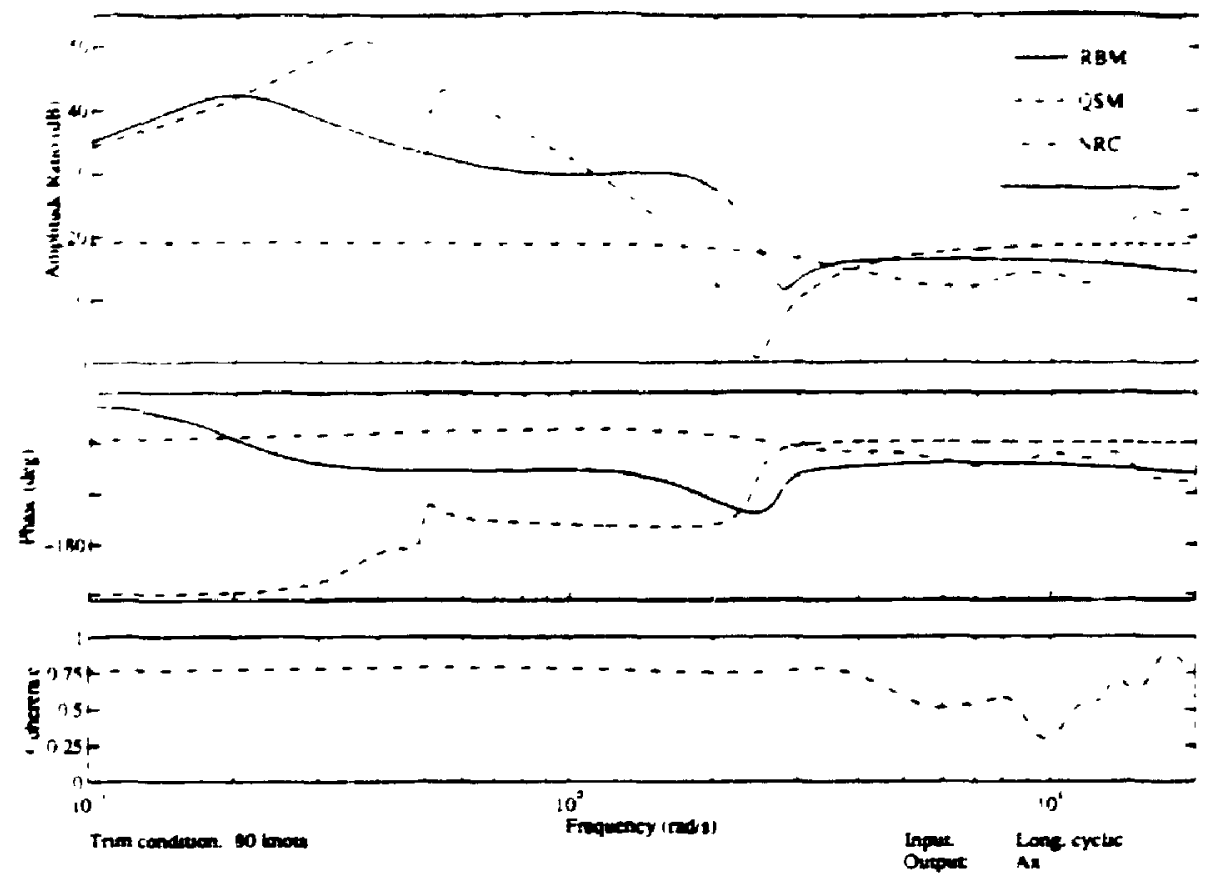

Figure 4.22: Longitudinal cyclic to forward acceleration at 80 knots

however at 80 knots they differ in the mid frequency range, the RBM model predicting a slightly better response in this range. The cause of the model prediction error at low frequencies is uncertain and due to the high coherence, nonlinearities can be ruled out. Is with the previous cases, an improved model prediction can be found by a reduction in body angle perturbations, figs. 4.23 and 4.24 .

The lateral cyclic to lateral acceleration response plots are presented in figures 4.25 to 4.27 . At hover, figure 4.25, there is good agreement between the RBM and QSM models. The predicted response is in good agreement with the flight test data at high frequencies and tends to break down in the mid to low frequency range. Both models predict a response which is higher in magnitude and $50^{\circ}$ out of phase with the NRC data. The response at 40 knots, figure 4.26 , shows a somewhat better magnitude prediction than the hover case but the high frequency phase (wher shifted by $360^{\circ}$ ) does not match as well as the QSM model does. At 80 knots, figure 4.27, good phase prediction is found bellow $2 \mathrm{rad} / \mathrm{s}$ and above $5 \mathrm{rad} / \mathrm{s}$, and the magnitude has the same shape but is offset by approximately $18 \mathrm{~dB}$. The effects of smaller body angle perturbations are shown in figs. 4.28 and 4.29 . 


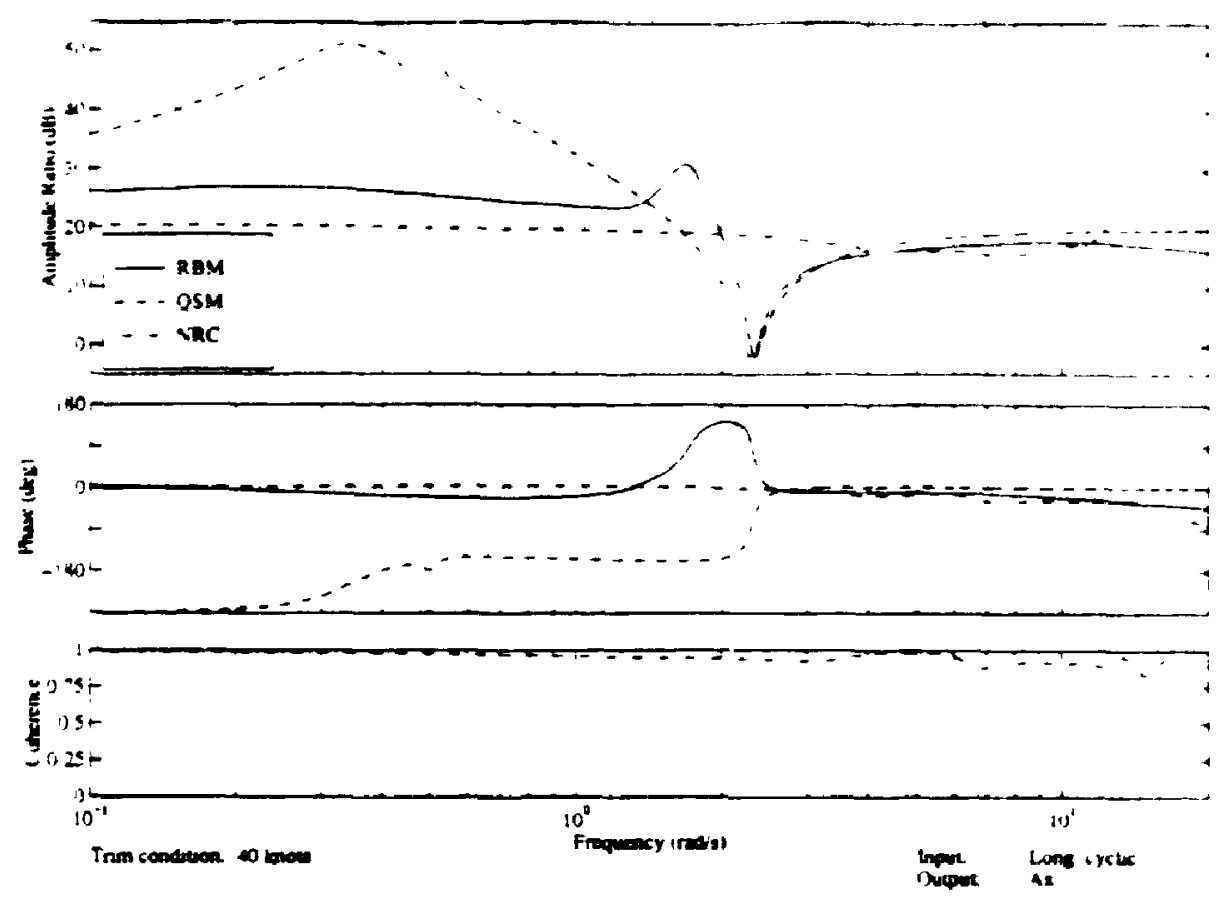

Figure 4.23: Longitudinal cyclic to forward acceleration at $40 \mathrm{knots}(\Delta 0=0.01 \mathrm{~d}(\mathrm{cg})$
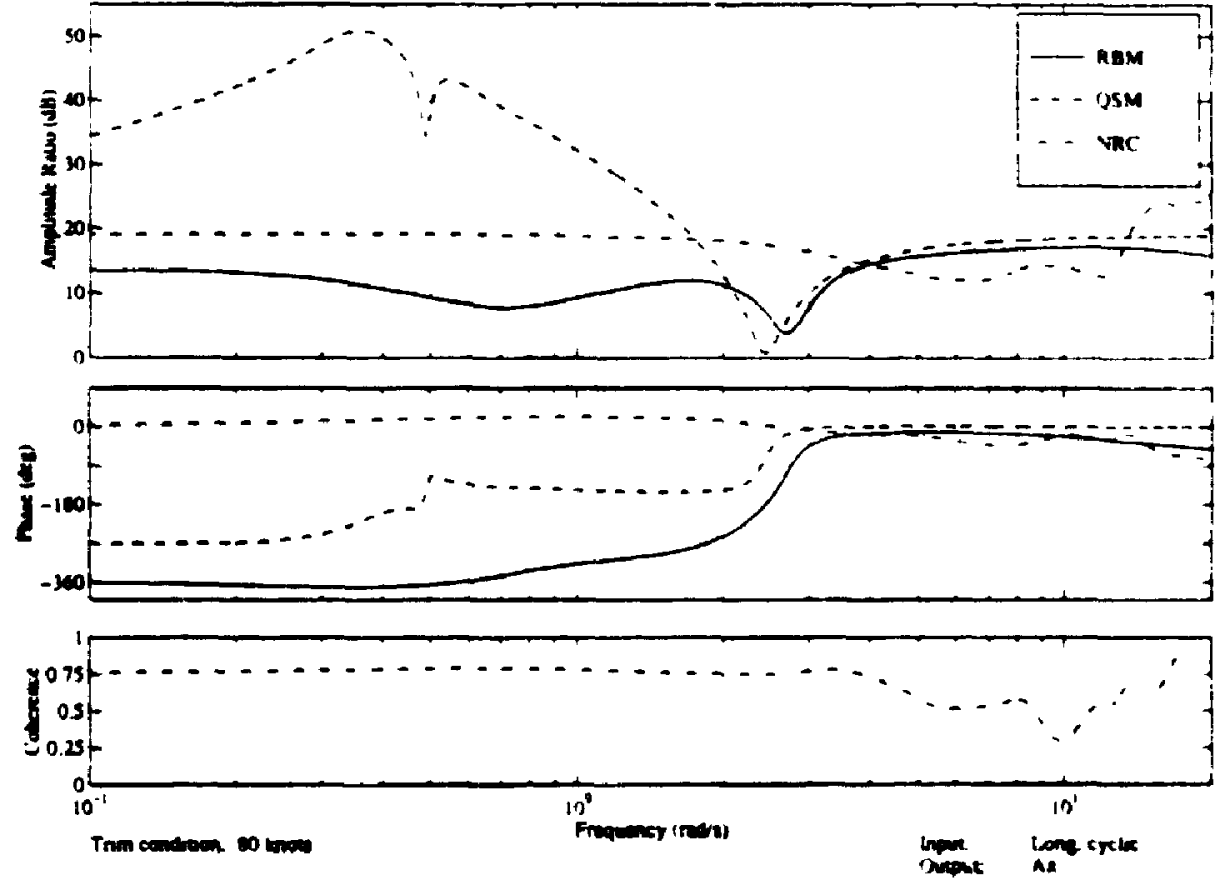

Figure 4.24: Longitudinal cyclic to forward acceleration at $80 \mathrm{knots}(\Delta \bar{\phi}=0.01 \mathrm{dag})$ 

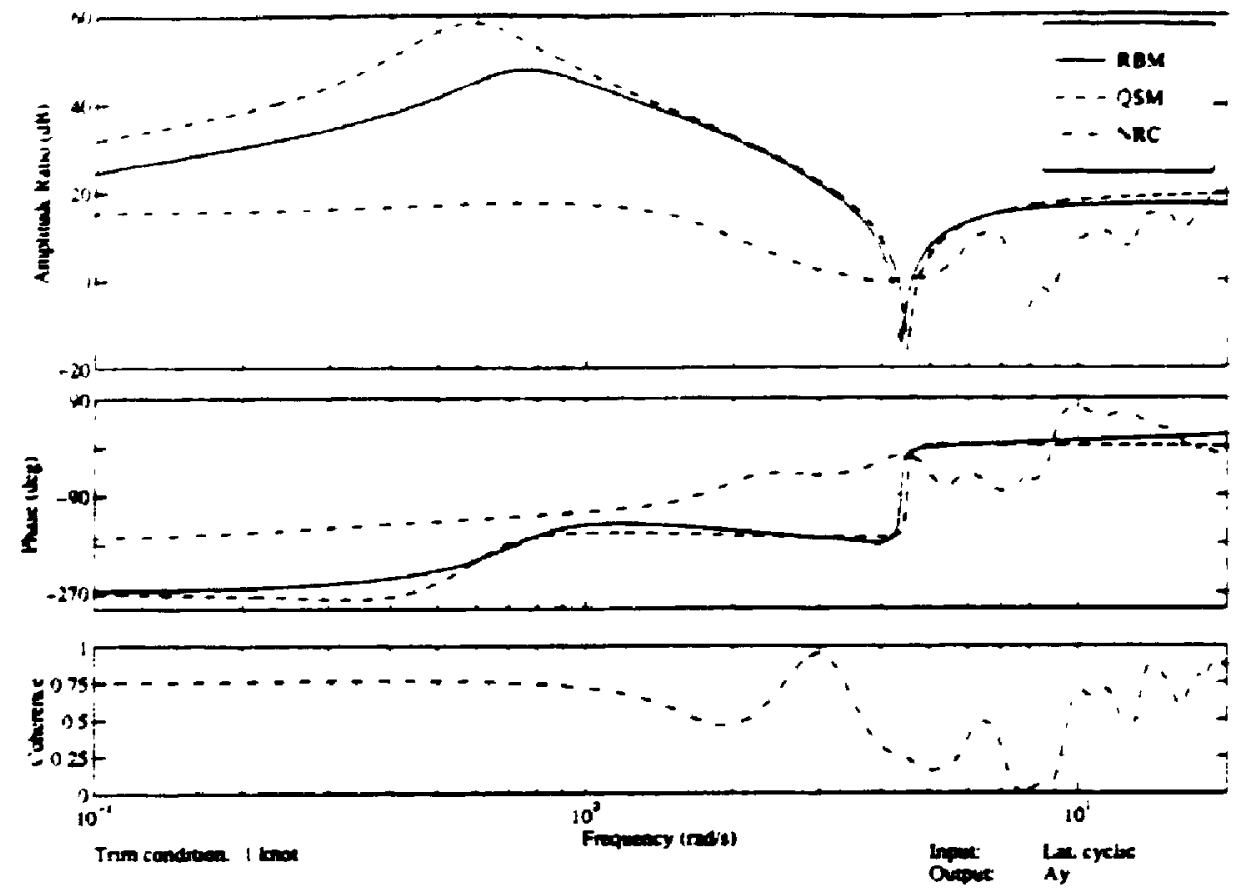

Figure 4.25: Lateral cyclic to lateral acceleration at hover
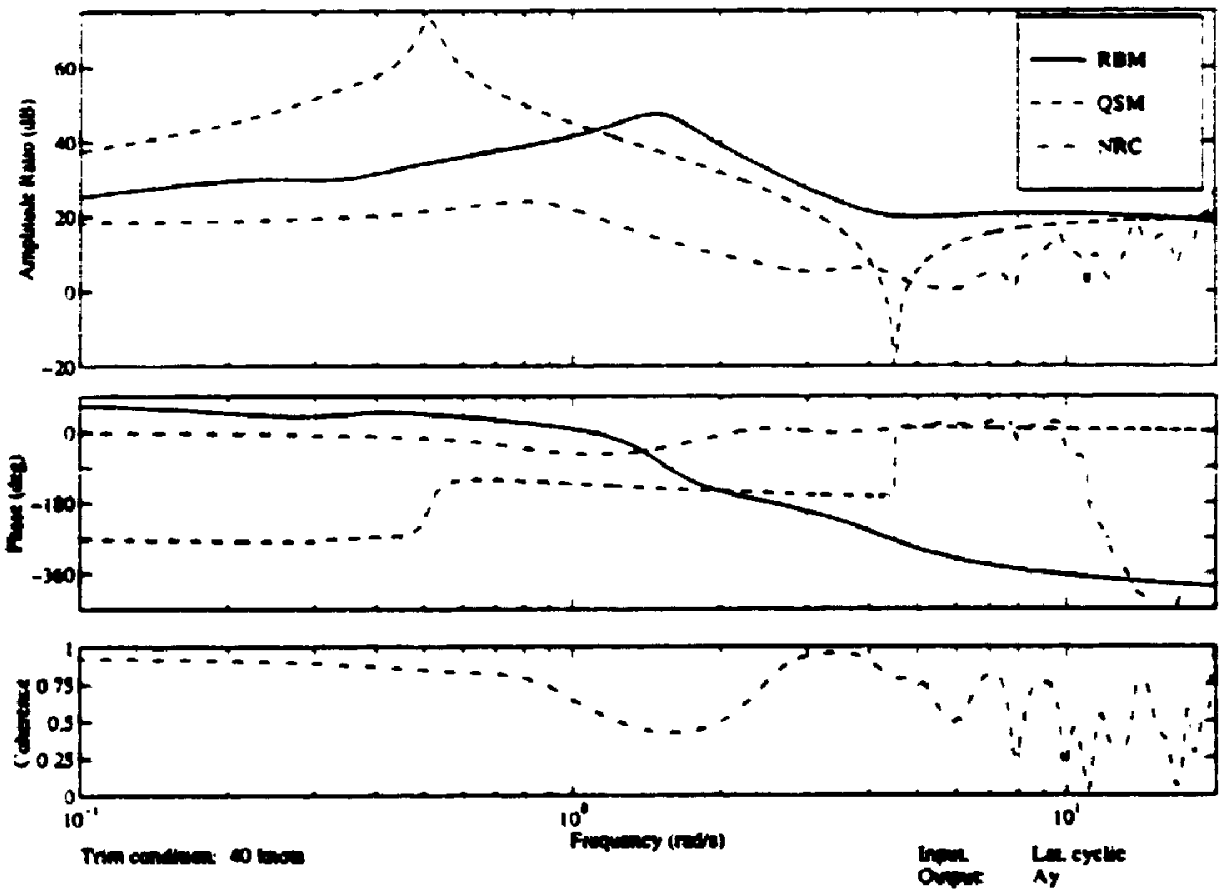

Figure 4.26: Lateral cyclic to lateral acceleration at 40 knots 
CHAPTER 4. MODEL IHLIDATION

$-1$
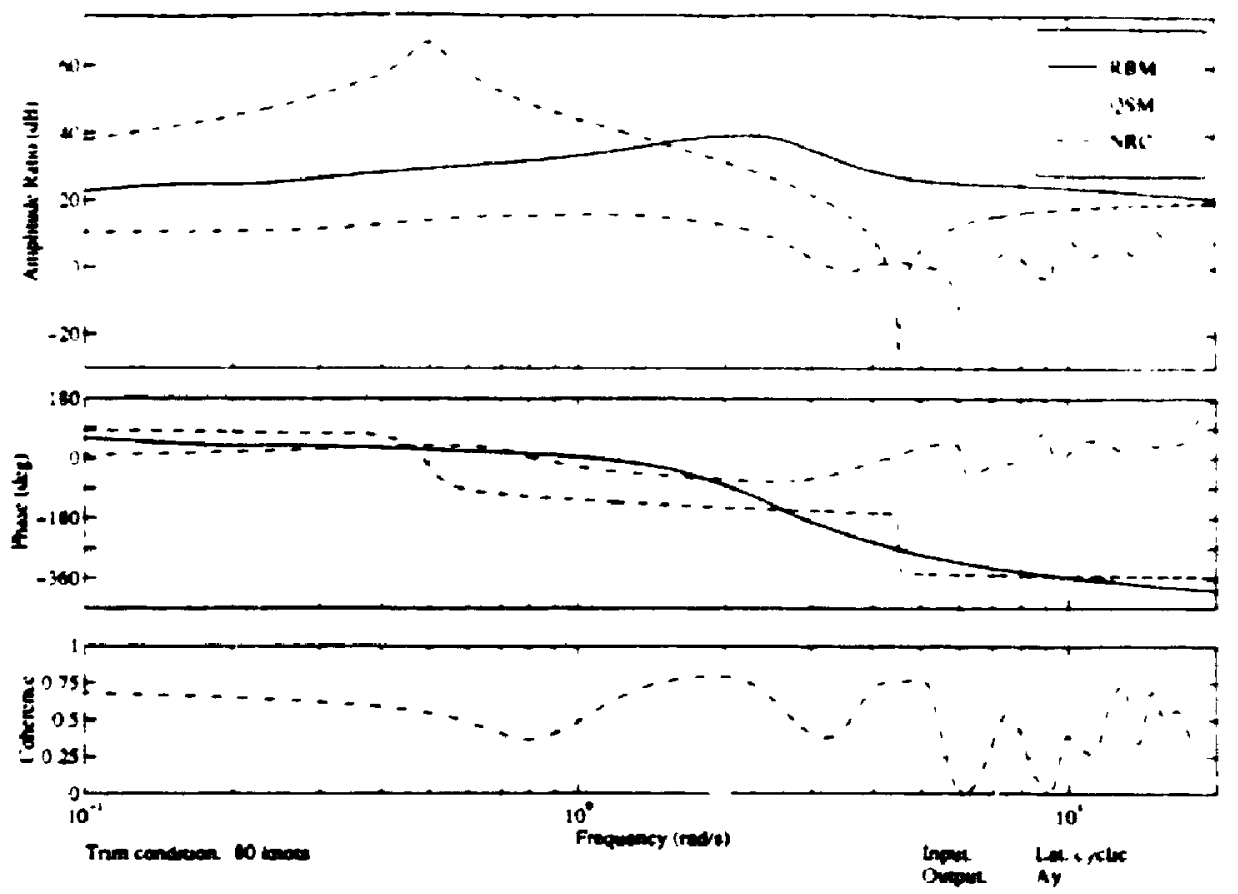

Figure 4.27: Lateral cyclic to lateral acceleration at 80 knots
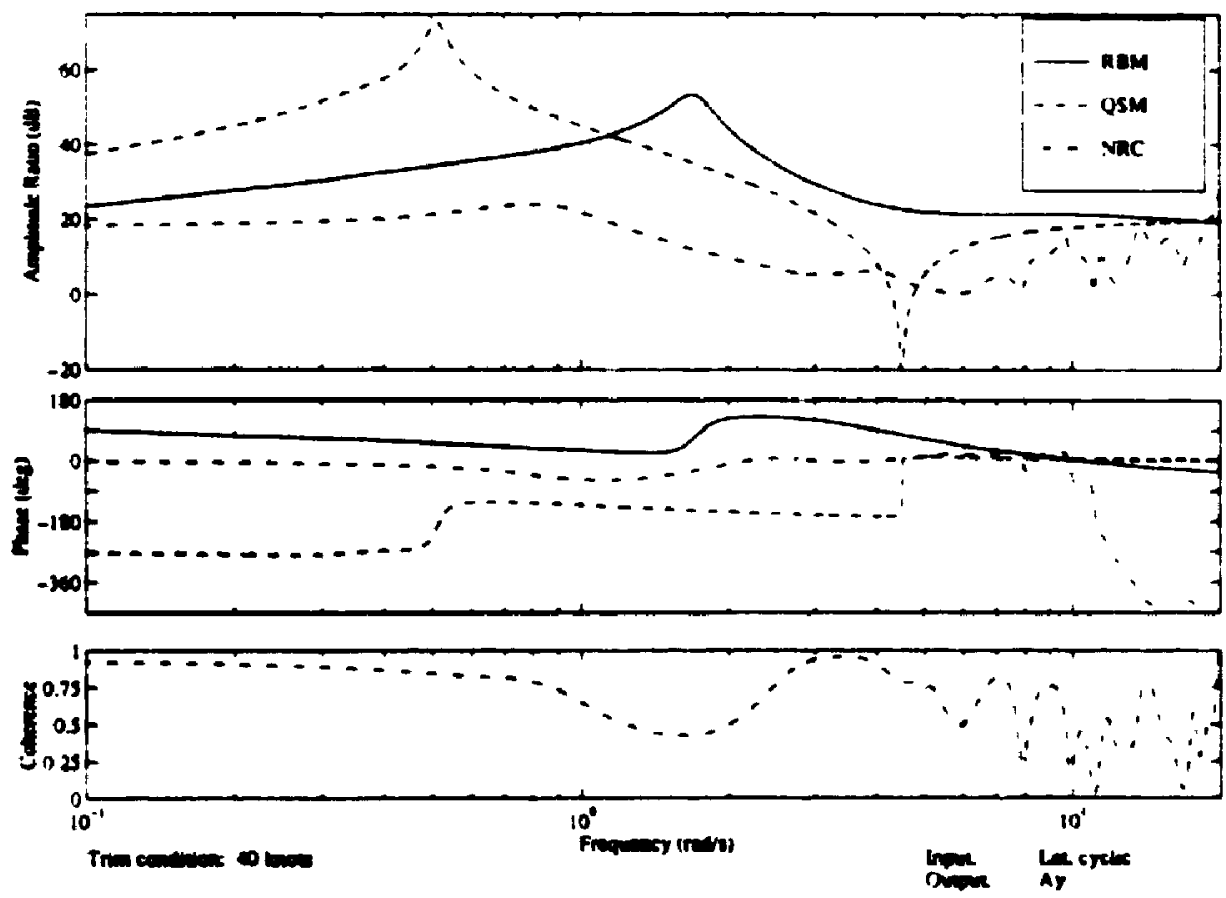

Figure 4.28: Lateral cyclic to lateral acceleration at 40 knots $(\Delta \bar{\phi}=0.01 \mathrm{deg})$ 


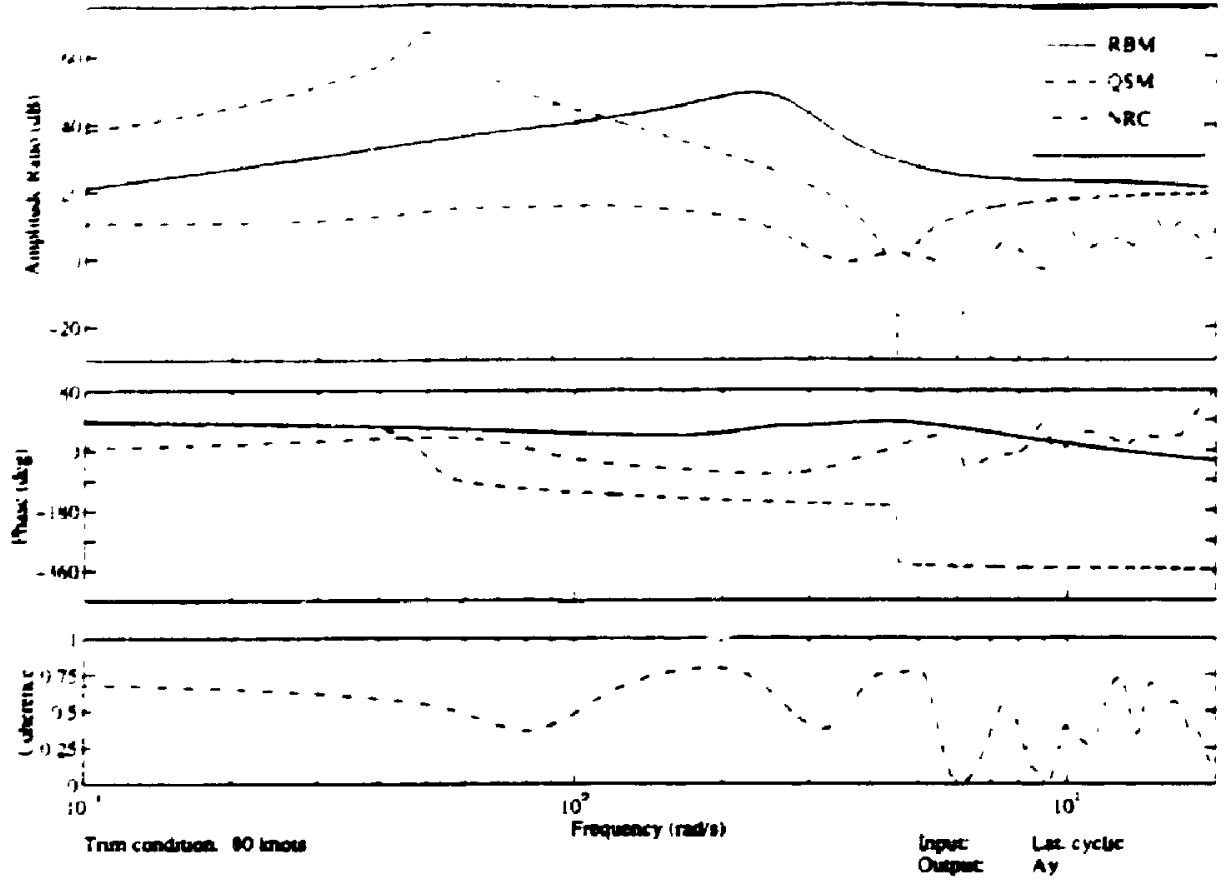

Figure 4.29: Lateral cyclic to lateral acceleration at $80 \mathrm{knots}(\Delta \bar{\phi}=0.01 \mathrm{deg})$

Lateral acceleration response from pedal inputs are plotted in figures $\mathbf{4 . 3 0}$ to $\mathbf{4 . 3 2}$. At hover. good agreement is found between the RBM and QS.M models. however. their predictions are poor in the mid to low frequency range. The high frequency phase and magnitude are in good arreement with the NRC data. however the low coherence of the data in this range makes comparison less meaningful. At both forward velocity trirn conditions the RBM response deviates from the QSM model at frequencies higher than $0.4 \mathrm{rad} / \mathrm{s}$ and does not provide an adequate high frequency match. The main reason for the poor match in forward flight is the lack of sidewash modeling at the tail rotor.

\subsubsection{Off-Axis Response}

The off-axis response of roll rate from longitudinal cyclic are shown in figures 4.33 to 4.35 . The RBM model is not in good agreement with the QSM model at any of the trim conditions, however, it better represents the true characteristics of the NRC Bell 205. This is believed to be the result of rotor dynamics excluded from the QSM model. During hover. figure 4.33. good agreement is found between the RBM model and NRC data at both high and low frequencies. The mid frequency response is deteriorated by two sharp 

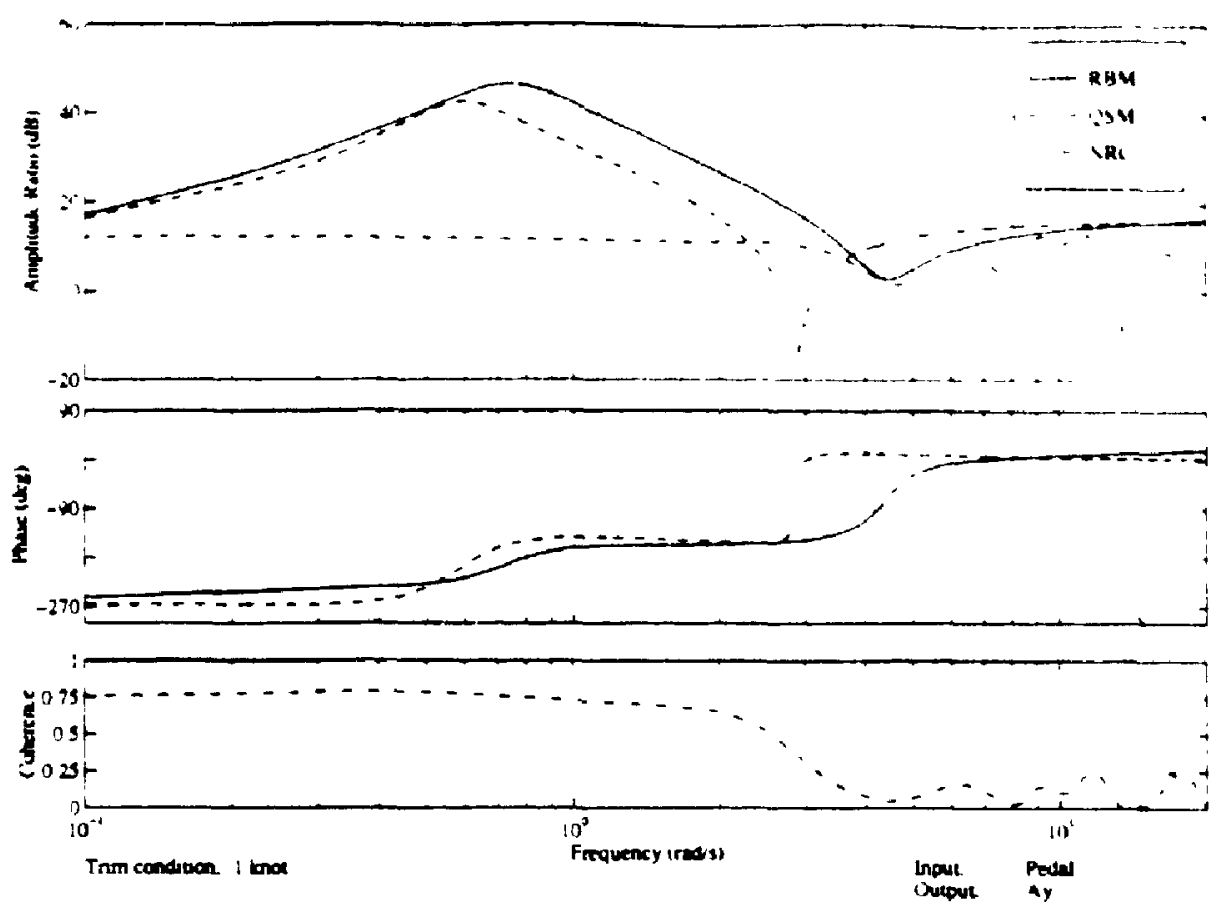

Figure 4.30: Pedal to lateral acceleration at hover
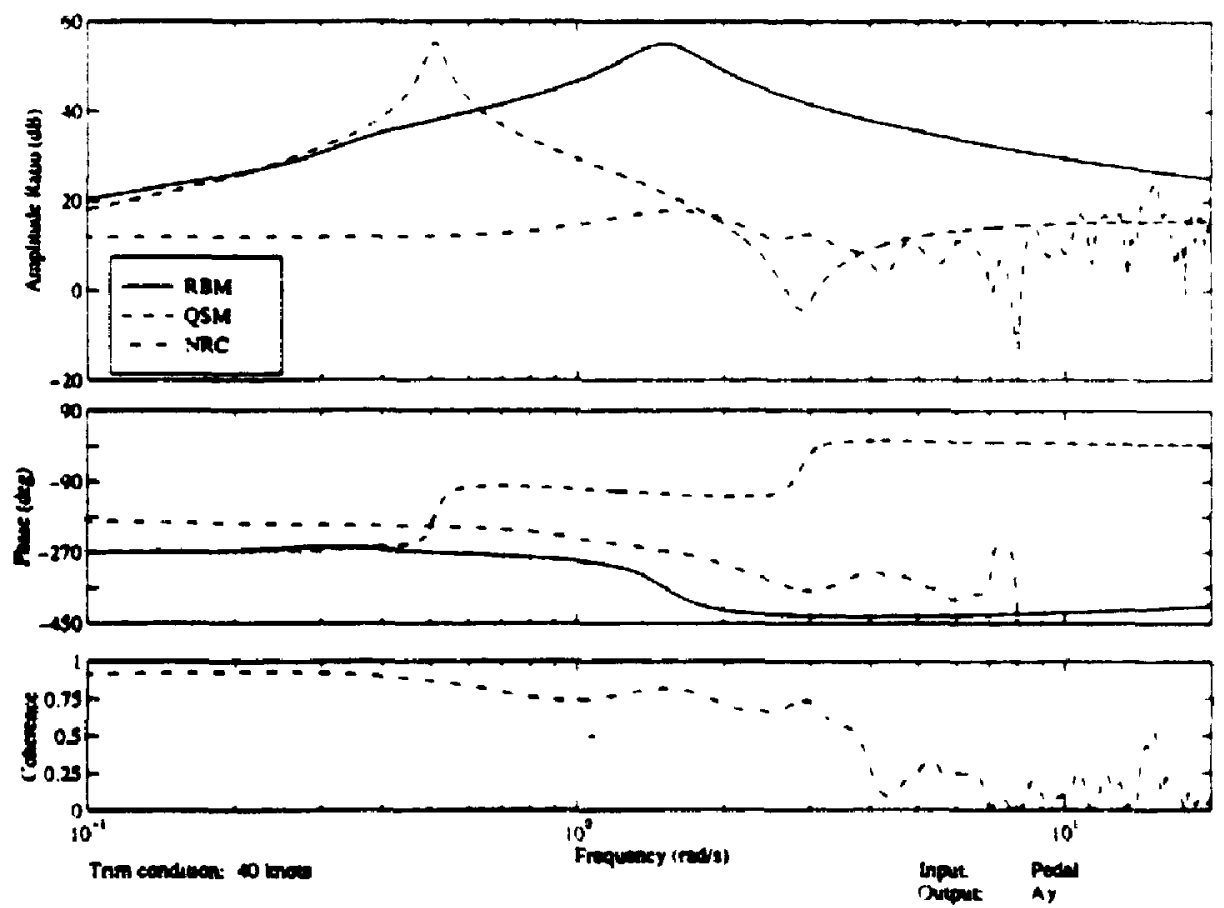

Figure 4.31: Pedal to lateral acceleration at 40 knots 


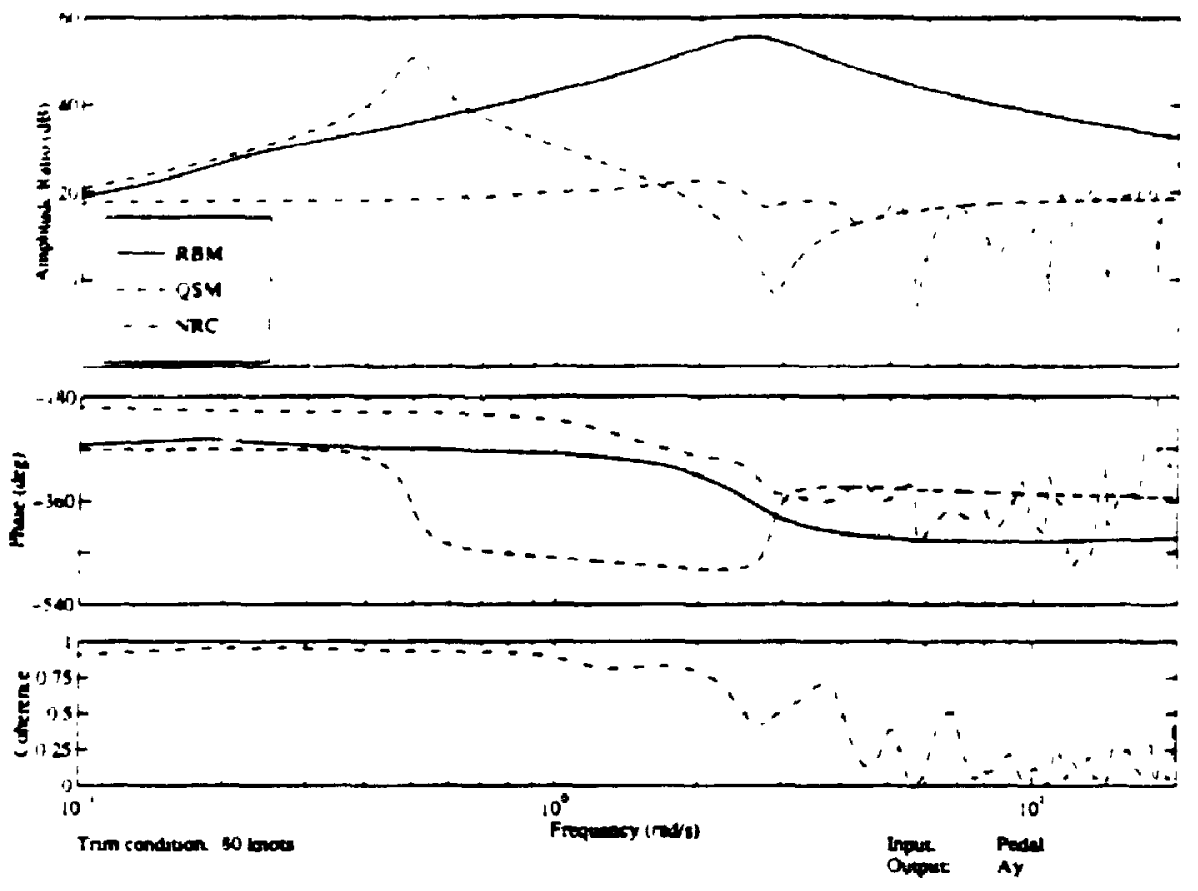

Figure 4.32: Pedal to lateral acceleration at 80 knots
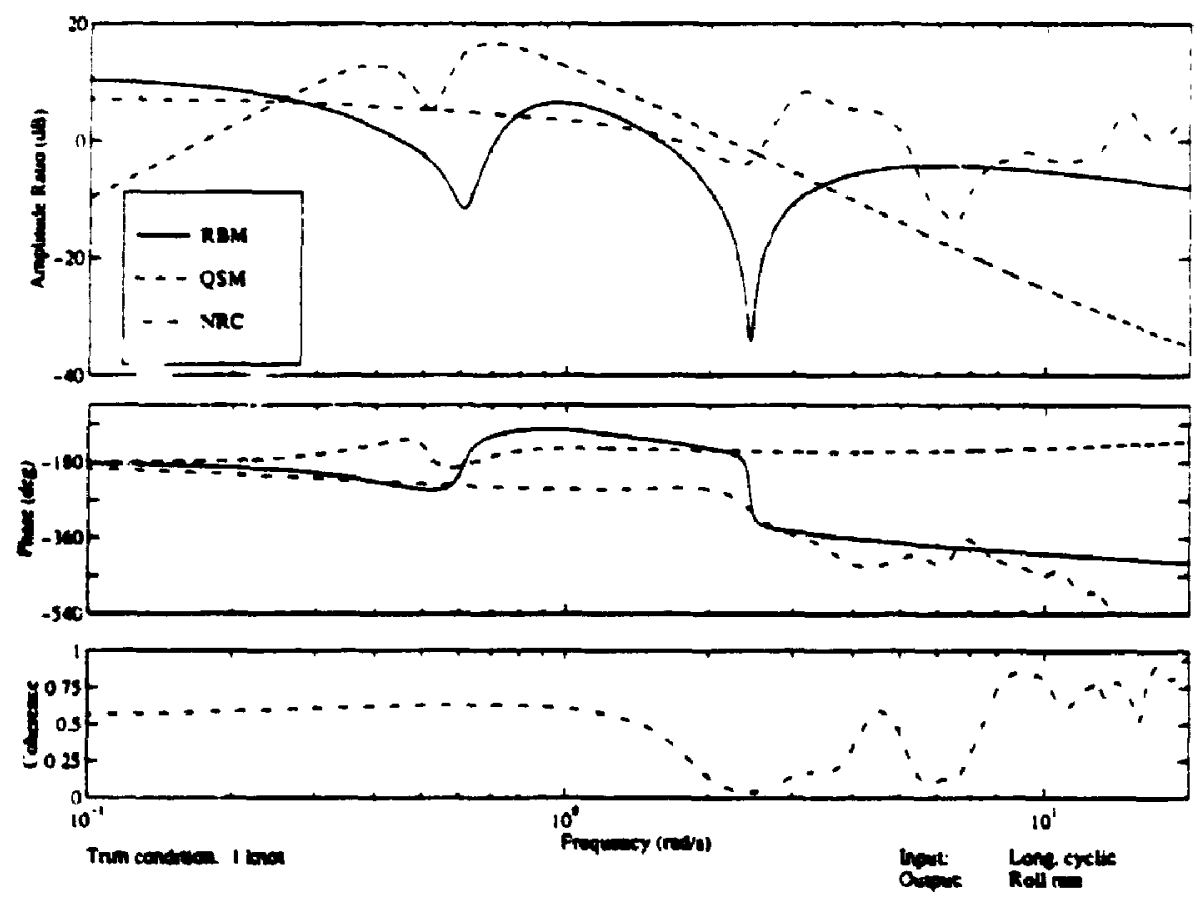

Figure 4.33: Longitudinal cyclic to roll rate at hover 


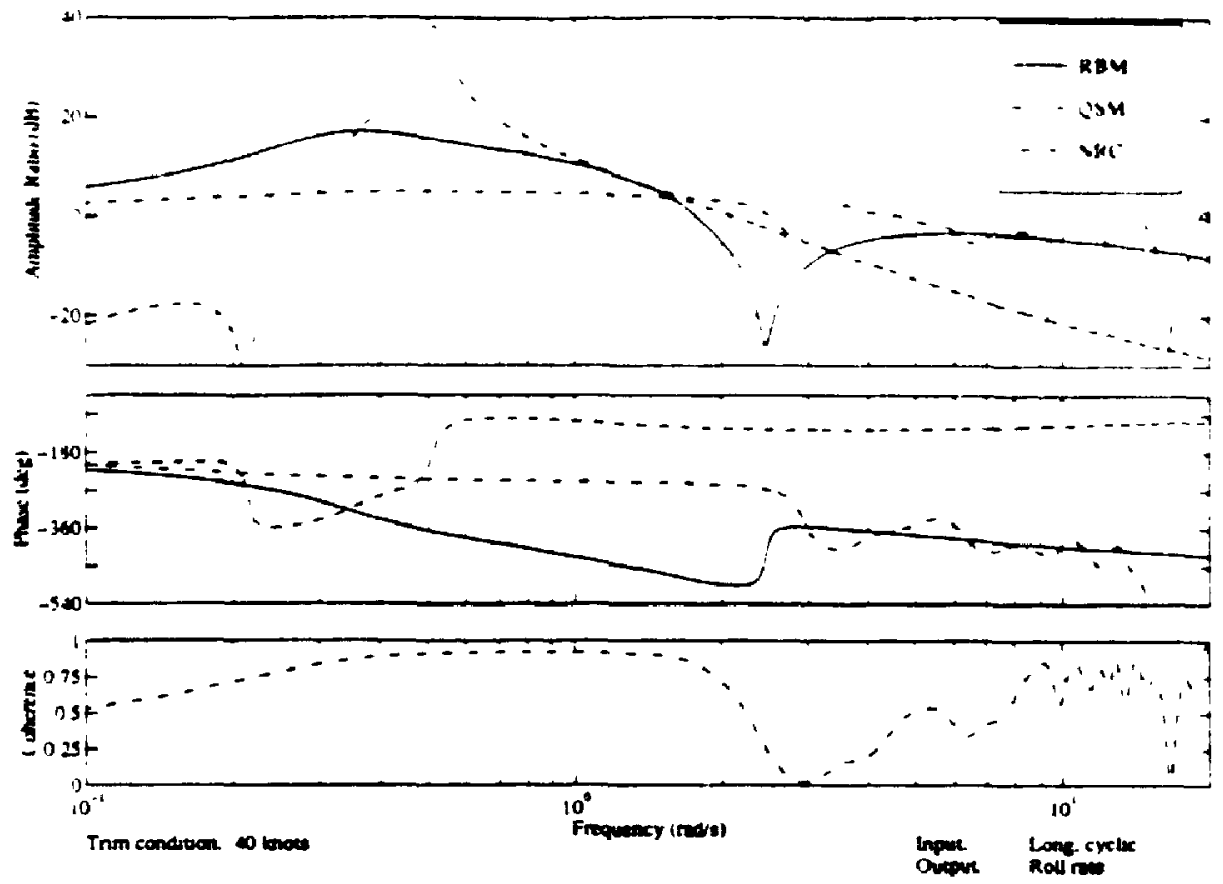

Figure 4.34: Longitudinal cyclic to roll rate at $\mathbf{4 0}$ knots

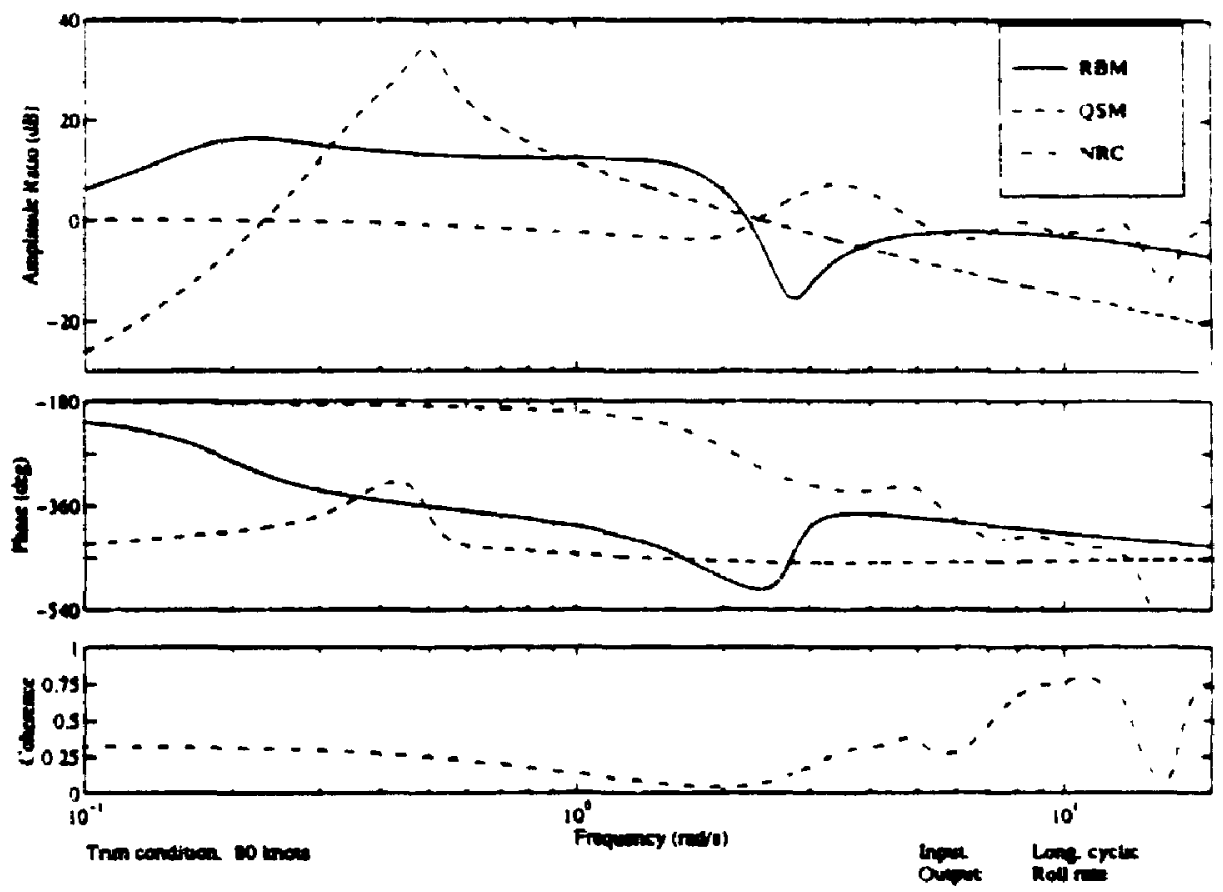

Figure 4.35: Longitudinal cyclic to roll rate at 80 knots 


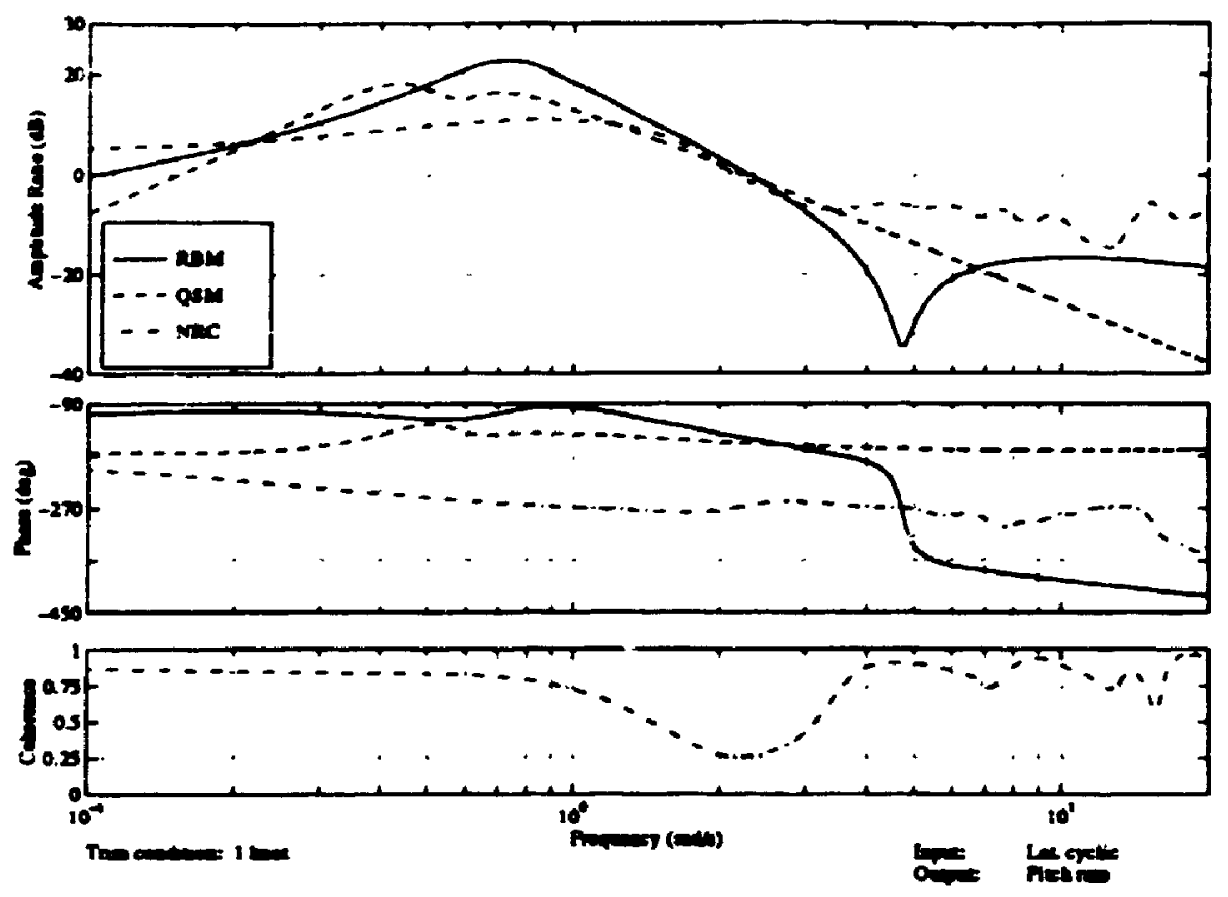

Figure 4.36: Lateral cyclic to pitch rate at hover

slope changes in magnitude which cause a corresponding $180^{\circ}$ phase shift. At 40 knots, figure 4.34, the model prediction is in good agreenent with the NRC data at frequencies above $2.5 \mathrm{rad} / \mathrm{s}$ and also at very low frequencies, breaking down elsewhere. At a forward speed of 80 knots, figure 4.35 , good agreement is found at frequencies above $3 \mathrm{rad} / \mathrm{s}$. In addition, the low frequency magnitude slope is in good agreement with the flight test data. Comparisons below $7 \mathrm{rad} / \mathrm{s}$ are less meaningful as the coherence is quite low.

The off-axis response of pitch rate from lateral cyclic are shown in figures 4.36 to 4.38 . As a general trend both RBM and QSM models predict the magnitude correctly below a frequency of $3 \mathrm{rad} / \mathrm{s}$. At frequenries above $6 \mathrm{rad} / \mathrm{s}$ only the $\mathrm{RBM}$ model correctly predicts the slope of the magnitude curve. Both models have a poor phase prediction throughout the frequency range of interest.

The collective to yaw rate response curves are plotted in figures 4.39 to 4.41 . The RBM and QSM models predict identical responses at hover. The predicted response does not match that of the flight test data, however, due to the low coherence of the flight test data, no conclusions can be drawn. At the 40 knots trim condition, figure 4.40 , the RBM and QSM models only agree at frequencies of $2 \mathrm{rad} / \mathrm{s}$ and up. As with the hover case, the 


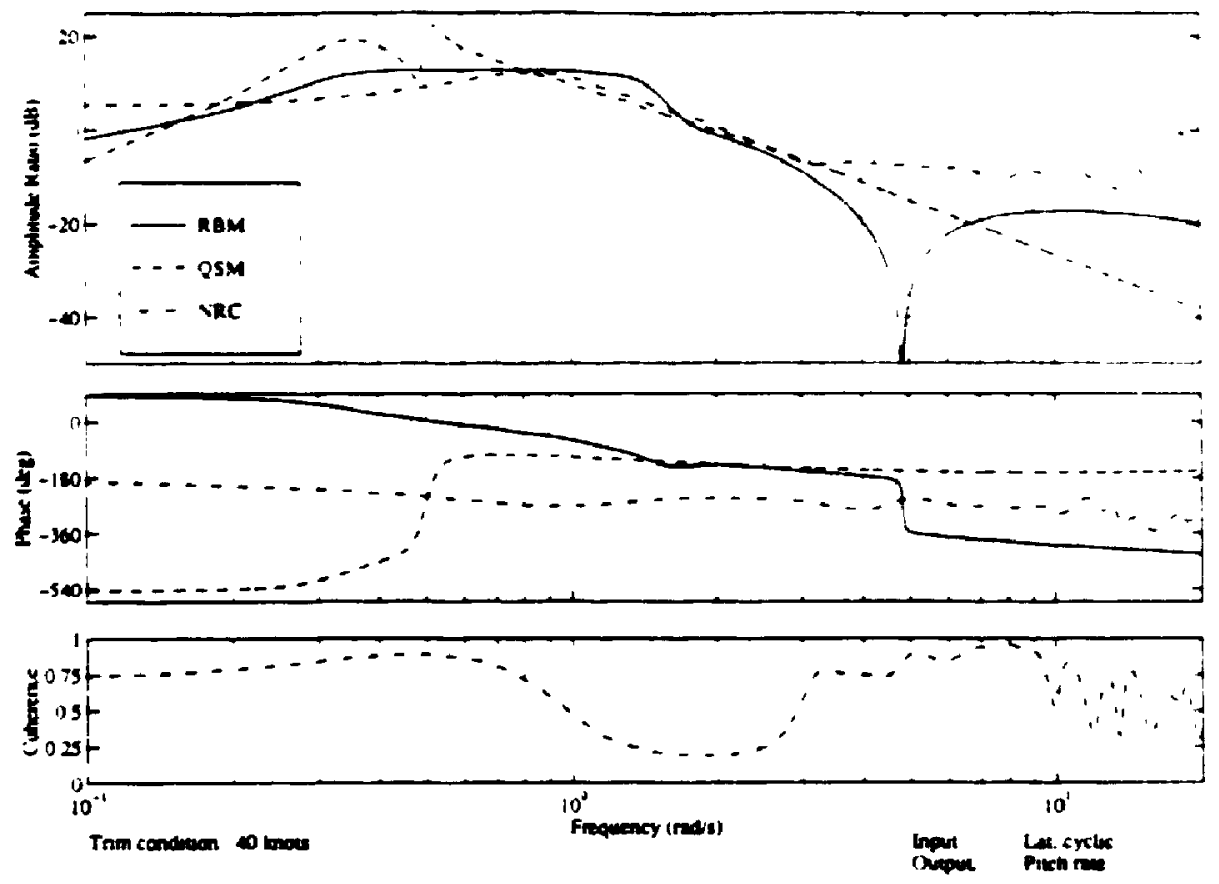

Figure 4.37: Lateral cyclic to pitch rate at 40 knots

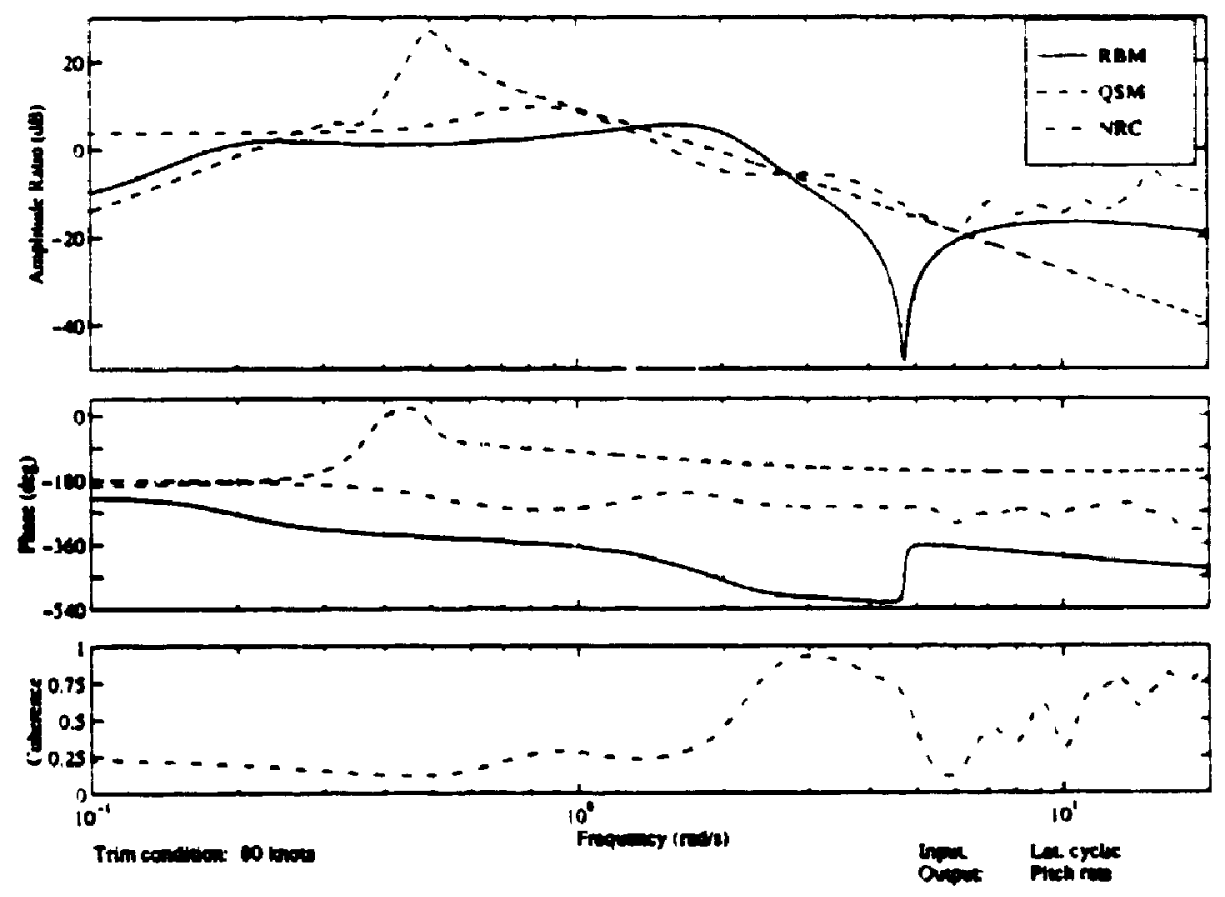

Figure 4.38: Lateral cyclic to pitch rate at 80 knots 


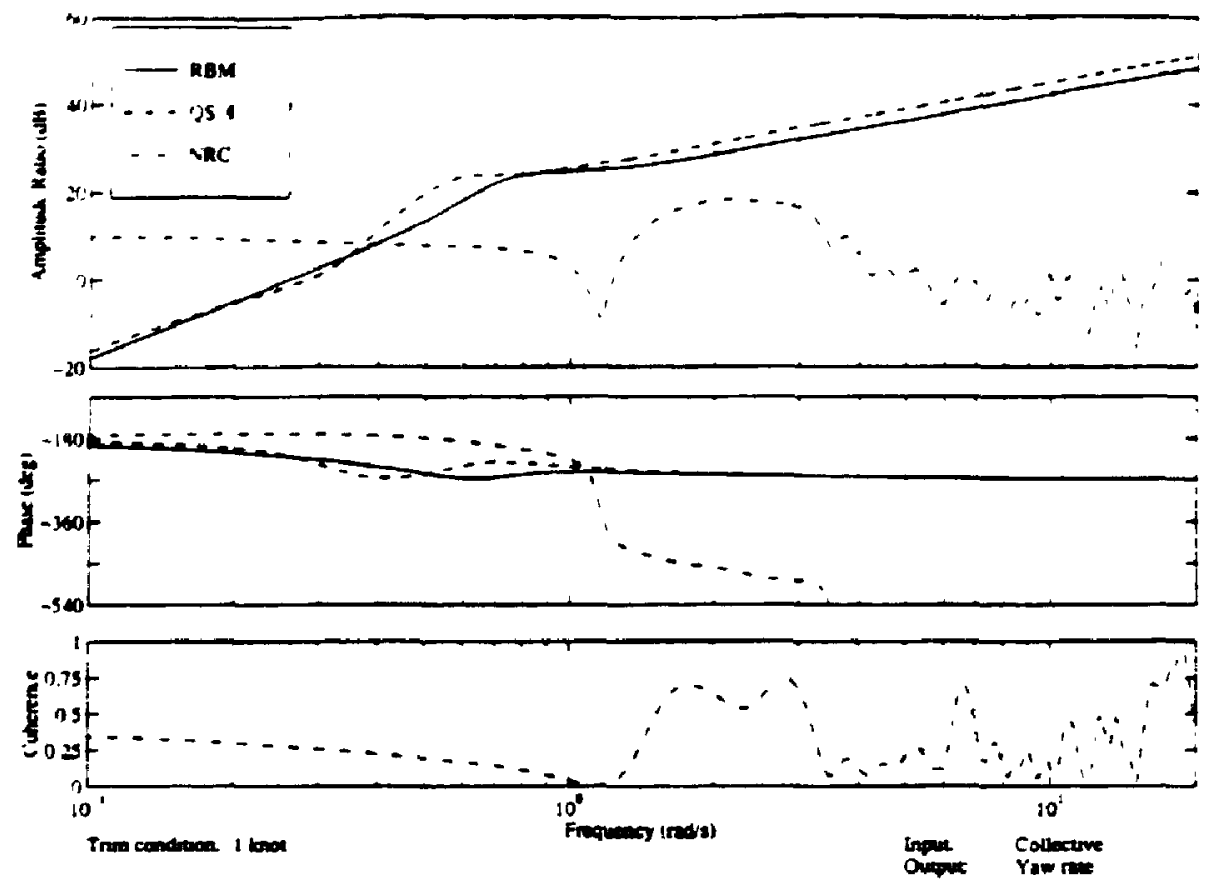

Figure 4.39: Collective to yaw rate at hover
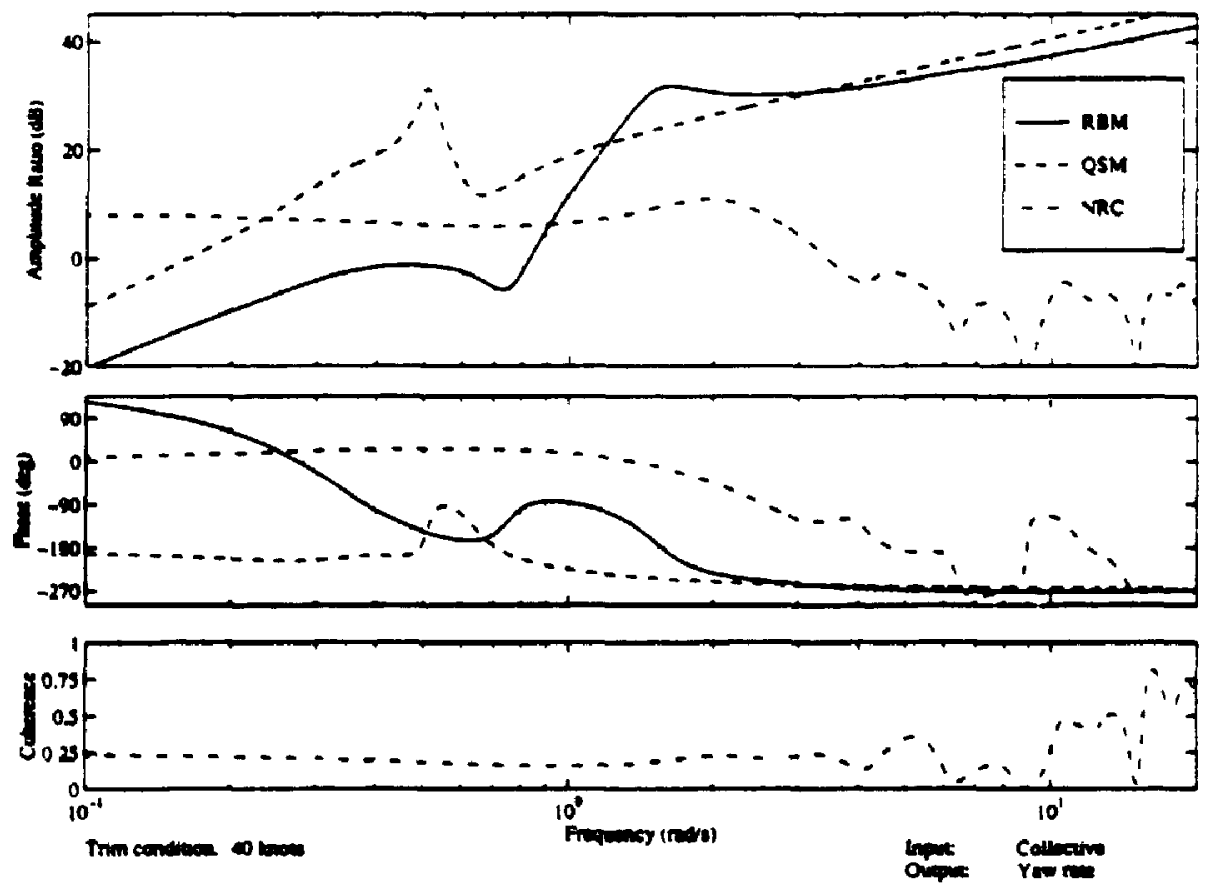

Figure 4.40: Collective to yaw rate at 10 knots 

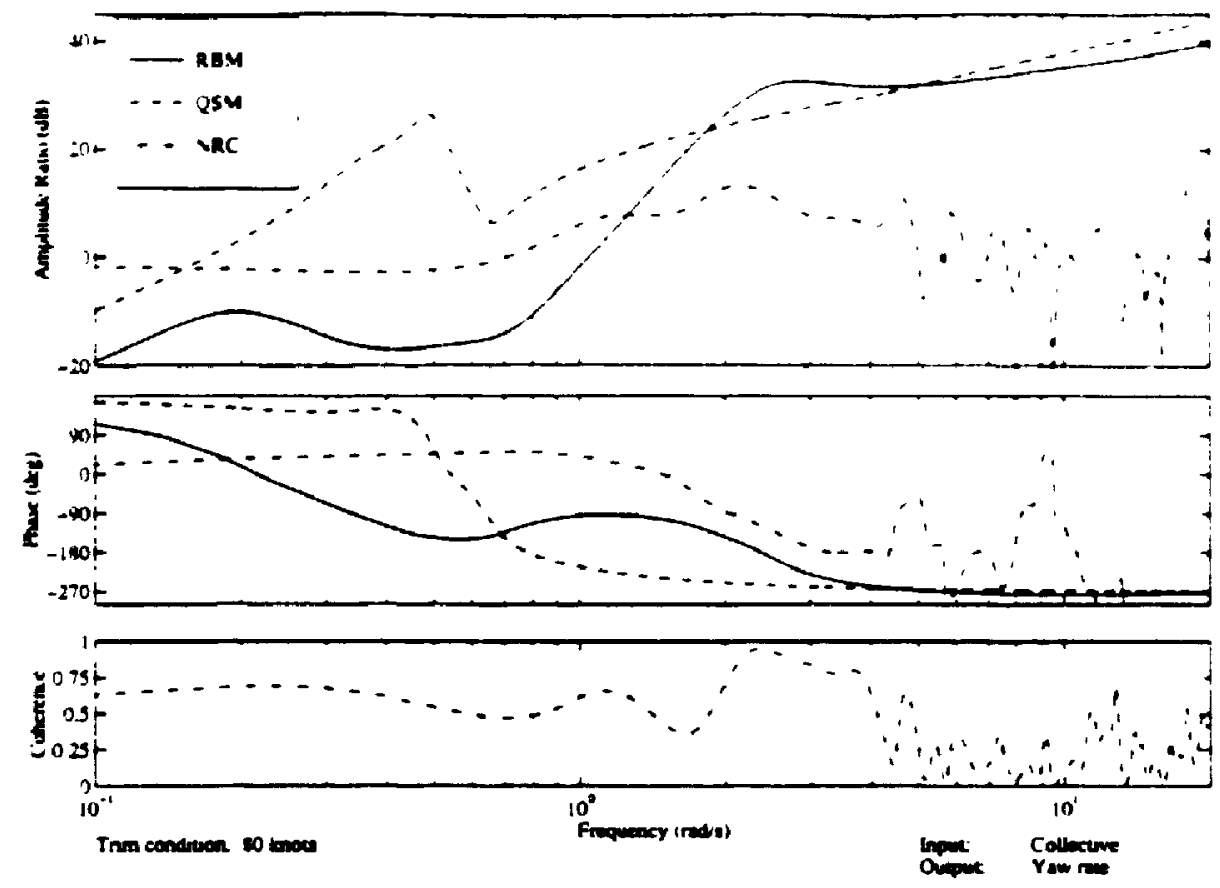

Figure 4.41: Collective to yaw rate at 80 knots

low coherence of the NRC data does not allow meaningful comparisons. The same general comments can be made for the 80 knots trim condition, figure 4.41 , however the coherence of the flight test data is high enough to make comparisons at frequencies bellow $4 \mathrm{rad} / \mathrm{s}$. Vieither of the models predict the response accurately.

\subsection{Main Rotor Force Transmission Modeling}

So far no attempt has been made to model the rotor-fuselage interface. It has been assumed that the forces produced by the rotor are directly transmitted to the fuselage through the main rotor shaft. This is however not the case as the flexible nature of the interface was seen to alter the response of the helicopter at frequencies above $10 \mathrm{rad} / \mathrm{s}$. The dynamic modeling of the rotor-fuselage interface is complex and is generally performed using modal techniques[27]. Due to the lack of data available to derive a model from first principles, a simple model is derived using frequency identification methods. Only the longitudinal model is documented as the process for the lateral case is similar.

The vertical, longitudinal, and lateral forces produced by the rotor are the thrust $H$-force, and $Y$-force respectively. The thrust is transmitted directly to the fuselage via a 

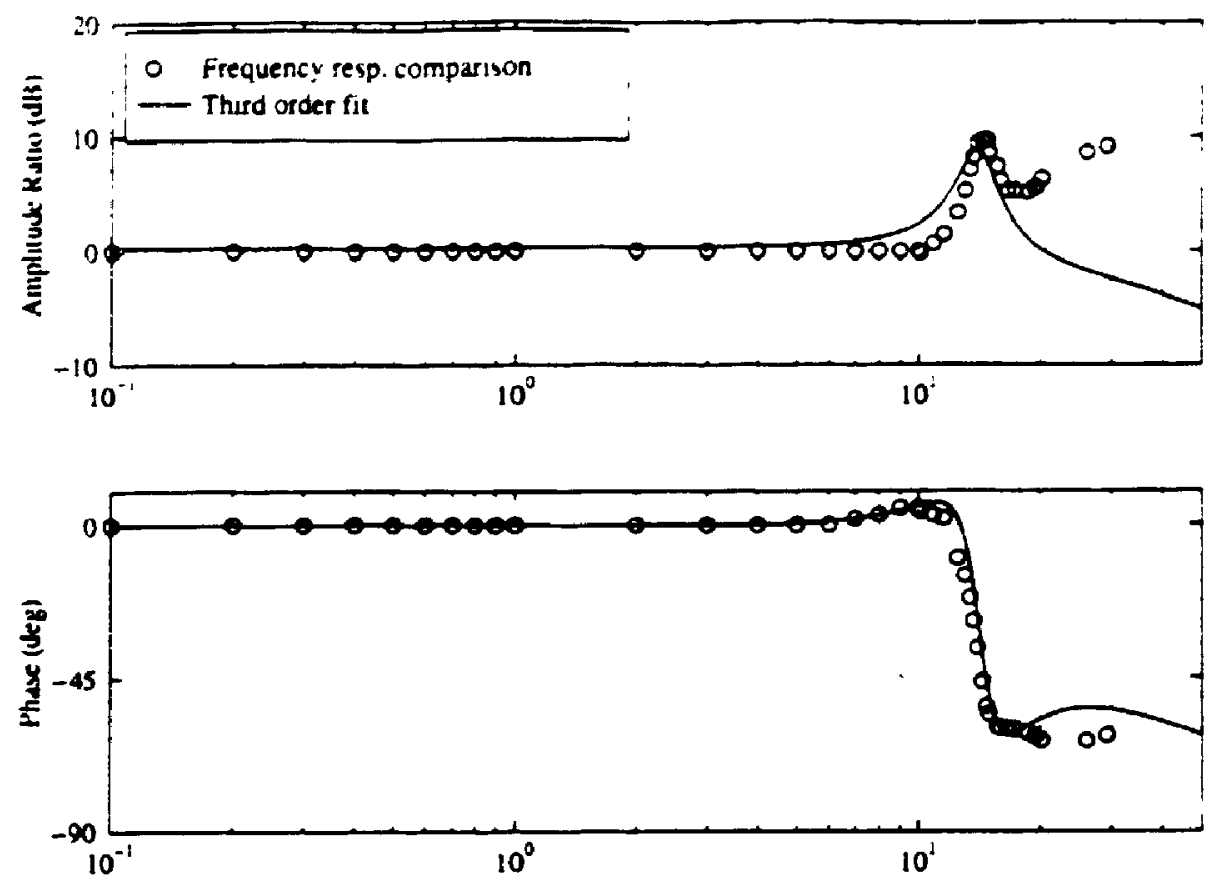

Figure 4.42: Longitudinal transmission mode

thrust bar and therefore only structural dynamics are involved which can be seen to have little effect upon the response of the helicopter from the vertical acceleration to collective input plots, figs. 4.15 to 4.19. However, the $H$ and $Y$ forces are not transmitted directly to the fuselage as the rotor-fuselage interface is flexible in these directions. The $\mathbf{H}$ and $\mathrm{Y}$ forces generated by the rotor are altered by the rotor-fuselage linkage and transmitted to the fuselage as $H_{e f f}$ and $Y_{\text {eff }}$. The dynamics of the linkage can be approximated by designing a filter which alters the response of the model to match that of the NPC flight test data at the dominant frequencies. The linkage dynamics in the longitudinal and lateral planes can be approximated from the on-axis pitch rate and roll rate responses respectively.

The difference between the RBM response and flight test data from figure 4.3 is plotted in figure 4.42. The points in the frequency range of 10 to $20 \mathrm{rad} / \mathrm{s}$ are directly from figure 4.3, the points below this range are set to zero in an attempt to limit the effect of the filter to the upper frequency region, and the points above $20 \mathrm{rad} / \mathrm{s}$ are chosen, somewhat arbitrarily, to shape the high frequency response of the filter.

The filter was designed with the "invfreqs" function of Matlab's signal processing 
toolbox ${ }^{[+3]}$ which is a complex-curve fitting function based on [5]. From the different order filters attempted. the third order filter provided the best match to the data shown as the solid line in figure 4.42 . The transfer function of this filter is:

$$
\frac{H_{. f f}}{H}=\frac{1}{1.0336} \frac{33.8317-8888 s^{2}+300.94697-8 s+i 234.473750}{s^{3}+38.45486251 s^{2}+299.0345856 s+6999.27171 .4}
$$

and is implemented in state-space form:

$$
H_{e f f}=\frac{1}{1.0336} c \bar{x}
$$

where $\bar{x}$ contains the transmission mode states and is defined according to:

$$
\begin{aligned}
\dot{\bar{x}} & =a \bar{x}+b H \\
a & =\left[\begin{array}{ccc}
-38.45486251 & -299.0345856 & -6999.271714 \\
1 & 0 & 0 \\
0 & 1 & 0
\end{array}\right] \\
c & =\left[\begin{array}{lll}
33.83177888 & 300.9469778 & 7234.475750
\end{array}\right]^{T} \\
b & =\left[\begin{array}{lll}
1 & 0 & 0
\end{array}\right]
\end{aligned}
$$

For linearizing purposes the trim states can be calculated by letting $\dot{\bar{x}}=0$ :

$$
\bar{x}_{0}=-a^{-1} b H_{0}
$$

Incorporating this filter into the non-linear computer simulation and linearizing ${ }^{5}$ about the hover trim condition gives rise to the frequency response plots shown in figures 4.43 to 4.45 , all others being unaltered.

\subsection{Trim Point Verification}

The trim states of the model can be compared to that of the flight test data and also to the AGAJ7407 version of the manufacturer's C81 Rotorcraft Flight Simulation Computer Program ${ }^{[27]}$. The linear QSM model ${ }^{[3]}$ used in the frequency response analysis is in fact developed by the $\mathrm{C} 81$ simulation program.

The C81 data presented in [3] is for a standard Bell UH-1H helicopter which differs from the NRC Bell 205A-1. The most significant difference between the two is the tail 

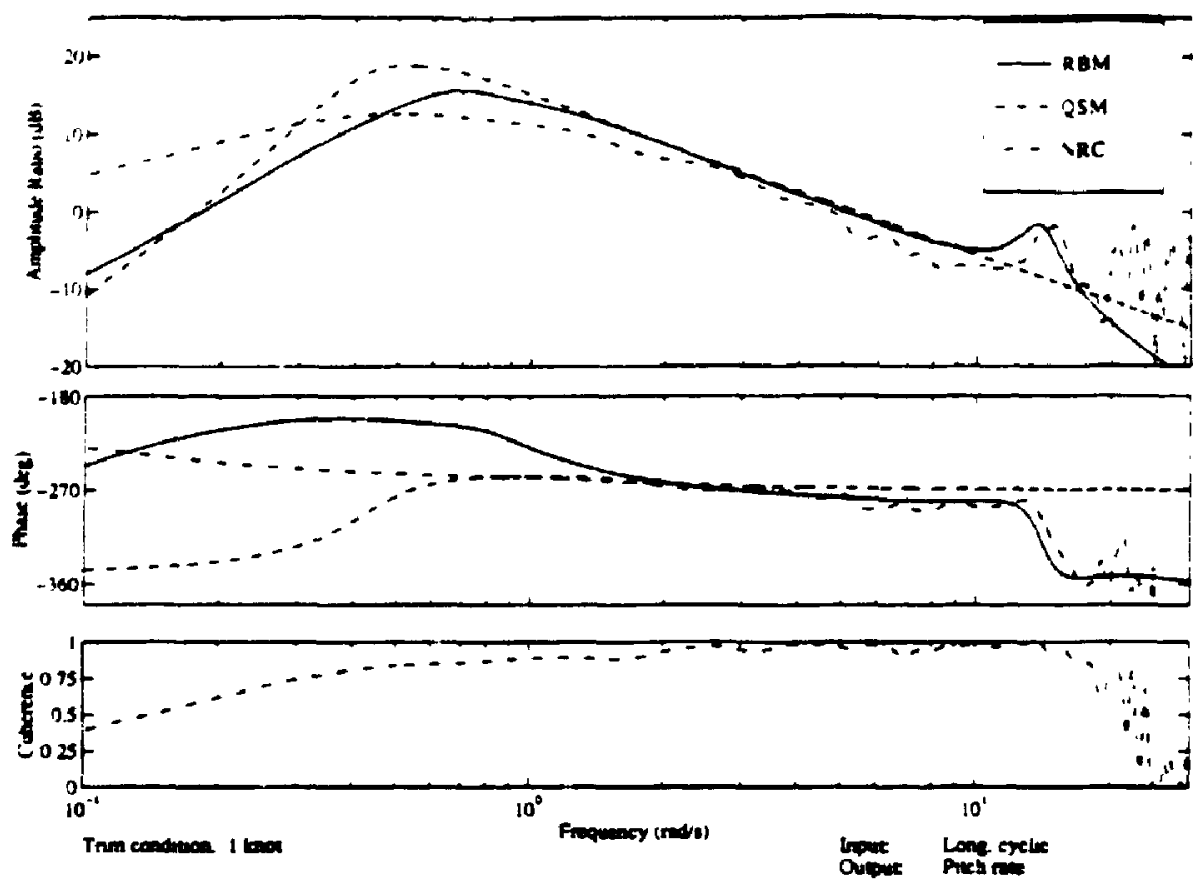

Figure 4.43: Longitudinal cyclic to pitch rate (hover)
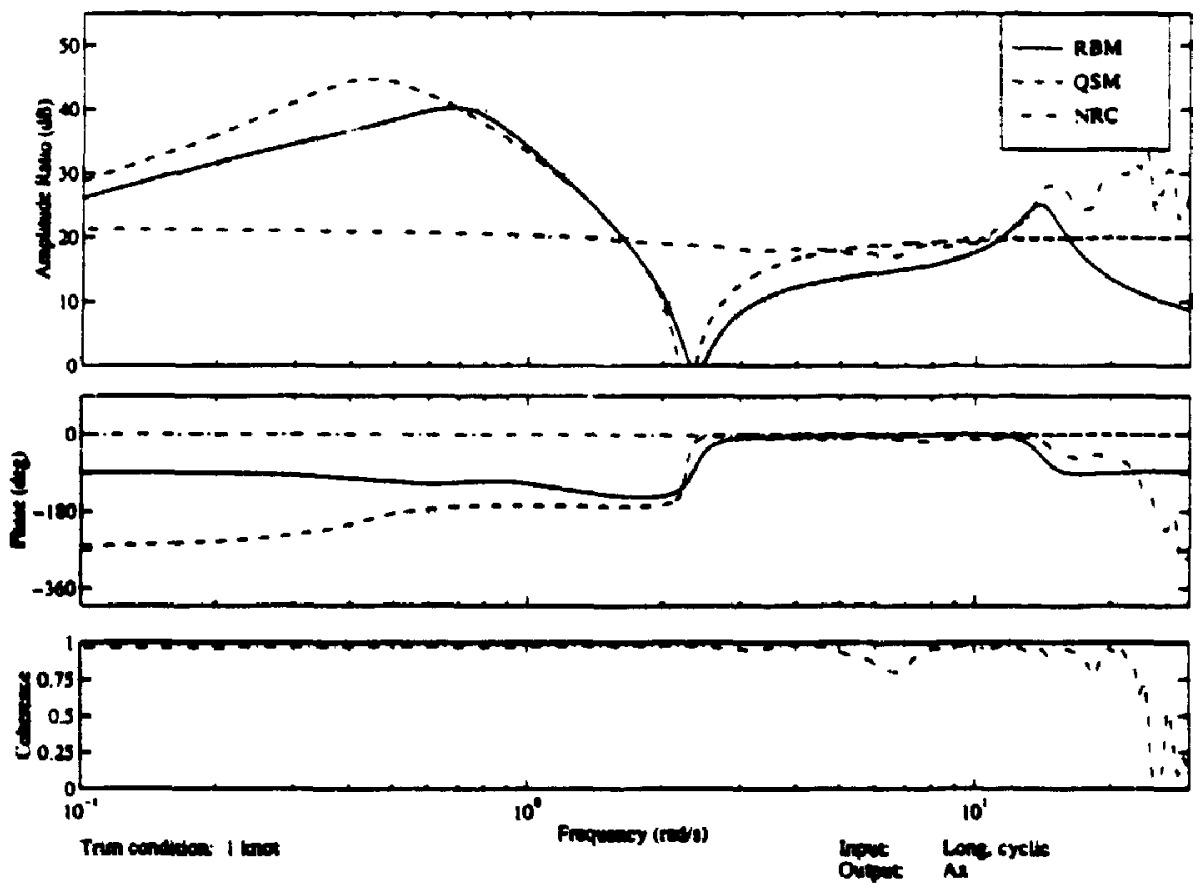

Figure 4.44: Longitudinal cyclic to forward acceleration (hover) 

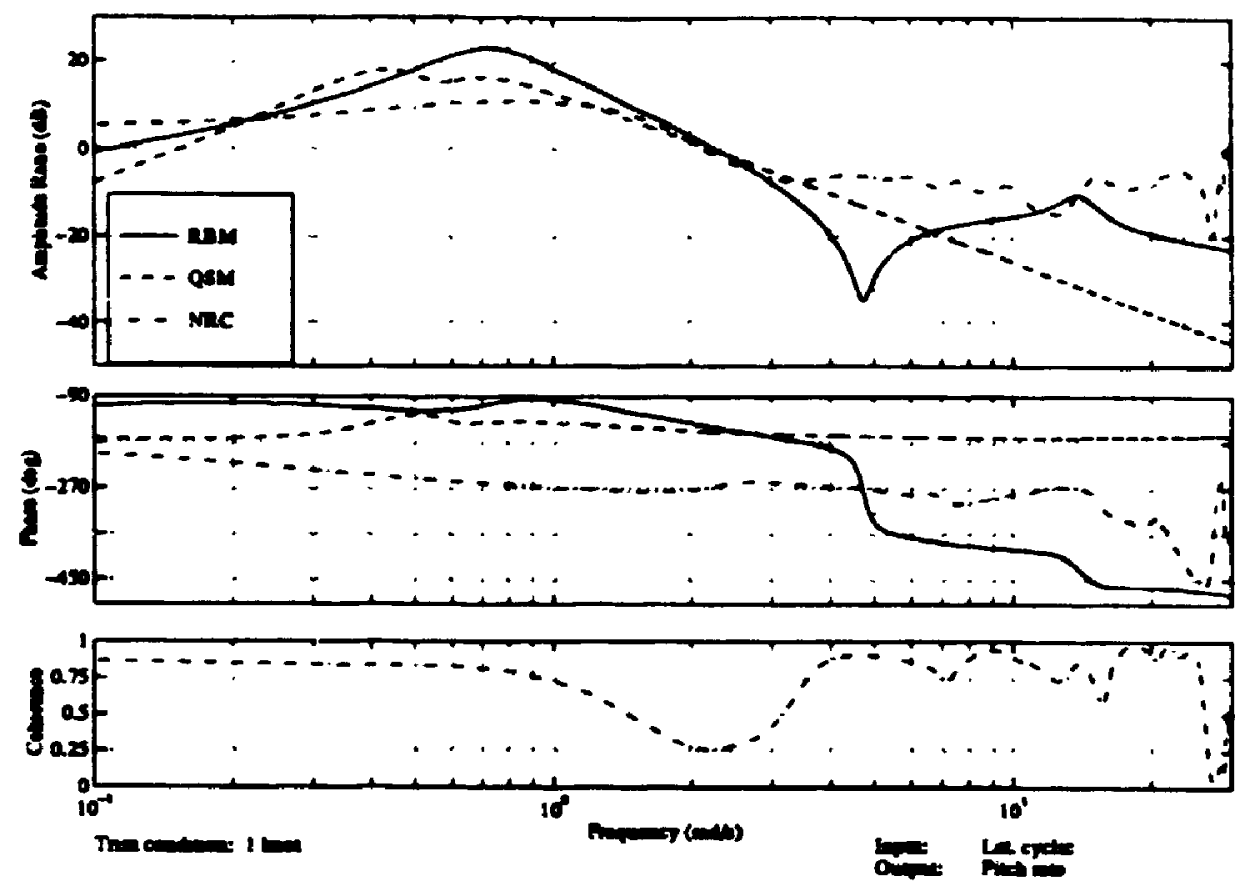

Figure 4.45: Lateral cyclic to pitch rate (hover)

rotor arrangement. The NRC Bell 205 has a right-side mounted tail rotor with a counter clockwise spin when viewed from the right side, whereas the UH-1H has a left-side mounted tail rotor and opposite spin. The direction of spin determines the direction of aerodynamic torque and thus effects the trim state of the helicopter (most evident in the longitudinal trim at hover). Trim comparisons between the RBM and C81 models are therefore performed with the tail rotor spin of the RBM model switched to match that of the C81 program. The tail rotor-vertical fin interaction is also different for the two configurations, however, this effect was assumed to be small and neglected. The trim states for the C81 and modified RBM models are presented in table 4.1 for the 1 knot, 40 knots, and 80 knots trim conditions. Good agreement is found between the two models, the greatest differences occurring at $\mathbf{4 0}$ knots where the complex main rotor wake impingement upon the stabilizer surfaces and the tail rotor becomes important.

Since the horizontal stabilizer-longitudinal cyclic gearing was not used during the Bell 205 flight tests, the incidence of the horizontal stabilizer was set to zero in the RBM before trim comparisons were made with the flight test data, as shown in table $4.2^{6}$. The trim

\footnotetext{
the $(\cdot)$ terms are derived using secondary measuremento
} 


\begin{tabular}{|c|c|c|c|c||c|c|}
\hline \multirow{2}{*}{} & \multicolumn{2}{|c|}{1 knot } & \multicolumn{2}{c|}{40 knot5 } & \multicolumn{2}{c|}{ 80 knots } \\
\cline { 2 - 7 } & RB.I & C81 & RBMI & C81 & RBM & C81 \\
\hline$\theta$ & 4.04 & 4.04 & 3.46 & 4.15 & 3.60 & 3.56 \\
\hline$\rho$ & -1.08 & -1.07 & -0.59 & -0.68 & -0.48 & -0.73 \\
\hline$u_{F}$ & 0.51 & 0.51 & 20.54 & 20.52 & 41.07 & 41.08 \\
\hline$v_{F}$ & 0.00 & 0.00 & -0.01 & -0.02 & -0.02 & -0.03 \\
\hline$w_{F}$ & 0.04 & 0.04 & 1.24 & 1.49 & 2.58 & 2.56 \\
\hline$\theta_{M R}$ & 10.54 & 10.35 & 9.23 & 9.10 & 9.10 & 9.14 \\
\hline$A_{s}$ & 0.57 & 0.49 & -0.01 & -0.40 & -0.20 & -1.60 \\
\hline$A_{c}$ & 1.12 & 1.04 & 1.14 & 1.66 & 0.95 & 0.97 \\
\hline$\theta_{T R}$ & 1.53 & 1.43 & 0.70 & 0.60 & 0.45 & 0.46 \\
\hline unit.
\end{tabular}

Table 4.1: Comparison of RBM and C81 trim states

\begin{tabular}{|c|c|c|c|c|c|c|}
\hline & \multicolumn{2}{|c|}{$1 \mathrm{knot}$} & \multicolumn{2}{|c|}{40 knots } & \multicolumn{2}{|c|}{80 knots } \\
\hline & RBM & NRC & RBM & NRC & RBM & NRC \\
\hline$\theta$ & 3.74 & 5.55 & 3.01 & $4.12(4.57)$ & 2.61 & $1.41(3.03)$ \\
\hline$\phi$ & -1.08 & -2.54 & -0.59 & 0.64 & -0.47 & 0.72 \\
\hline$u_{\mathbf{r}}$ & 0.51 & 0.35 & 20.54 & 22.86 & 41.11 & 38.71 \\
\hline$v_{f}$ & 0.00 & 0.00 & -0.01 & -0.52 & -0.02 & 6.10 \\
\hline$w_{f}$ & 0.03 & -0.06 & 1.08 & 1.83 & 1.87 & 2.13 \\
\hline$\theta_{M R}$ & 10.54 & -1.14 & 9.22 & -2.65 & 9.09 & -2.28 \\
\hline A, & 0.73 & -1.37 & 0.23 & $\cdot 2.26$ & -0.65 & -3.3 \\
\hline$A_{c}$ & 1.12 & -0.07 & 1.14 & -0.74 & 0.95 & -0.94 \\
\hline$\theta_{T A}$ & 1.53 & 1.17 & 0.70 & 0.36 & 0.45 & 0.16 \\
\hline
\end{tabular}

Table 4.2: Comparison of RBM and NRC Bell 205 trim states 


\begin{tabular}{|c|c|c|c|c|}
\hline & \multicolumn{2}{|c|}{$1 \mathrm{knot}$} & \multicolumn{2}{|c|}{10 knots } \\
\hline & RBII & $\therefore R C$ & RB.II & SRC \\
\hline$\theta_{M R}$ & 10.54 & 10.23 & 9.22 & 3.82 \\
\hline$A_{s}$ & 0.73 & 1.28 & 0.23 & 0.39 \\
\hline$A=$ & 1.12 & 1.82 & 1.14 & 1.15 \\
\hline$\theta_{\tau R}$ & 1.53 & 1.46 & 0.70 & 0.68 \\
\hline
\end{tabular}

Table 4.3: Control trim comparison with zero shift

values of the flight test data are obtained by averaging the first portion of the state traces presented in appendix B. The attiiudes in table 4.2 are in fair agreement given that the center of gravity position of the NRC Bell 205 may be different from that used in the simu. lation. However, the control positions are considerably different. Although the calibration for the control settings are found to be accurate from the frequency response analysis. their zero positions are suspect. For example, the negative collective stick required to trim the helicopter does not make physical sense. Table 4.3 shows the 1 knot and 40 knots control trim values of table 4.2 with the VRC control positions shifted by the difference found in the 80 knots data. This results in a good all around match at $\mathbf{4 0}$ knots and also at 1 knot in the collective and pedal inputs. The discrepancy in the 1 knot longitudinal and lateral cyclic inputs may be due to the difficulty experienced in reaching trim during hover. However more data must be analyzed in order to make firm conclusions as to the proper zero setting of the controls.

\subsection{Summary}

Frequency response plots were used to compare the dynamic response of the model to that of the NRC flight test data and also to the QSM model. It was shown that the RBM model performs as well and in some instances better than the QSM model in predicting the body motions of the Bell 205 helicopter. The yaw response in forward flight is, however, inadequate below $1.1 \mathrm{rad} / \mathrm{s}$ and should not be used for design where the low frequency region is of importance. Frequency-domain identification techniques were used to model the flexible rotor-fuselage interface in the longitudinal plane, thereby extending 
the model validity to frequencies of $20 \mathrm{rad} / \mathrm{s}$. Trim points were also compared showing good agreement. 


\section{Chapter 5}

\section{Conclusions and Recommendations}

\subsection{Conclusions}

The goal of this thesis was to provide a high order linear model. with both the rotor and fuselage degrees of freedom, to replace the model presently used at the Flight Research Laboratory of the NRC. The motivation behind such a change is the need for higher order dynamics which are necessary for the design of high-gain feedback control systems. Before the RBM model can be adopted by the $\mathrm{NRC}$ it is necessary to prove that it represents the Bell 205 at least as weli as the QSM model.

The analysis of the previous chapters show that the RBM model can be used to represent the body states of the Bell 205 as well as, and in some instances, better than the model presently being used. The only exception to this is the yaw channel which is only reliable at low speeds. The yaw model does not perform adequately at frequencies below $2.5 \mathrm{rad} / \mathrm{s}$ in forward flight and therefore can not be relied upon for the design of feedback control systems. This deficiency is due the unmodeled sidewash experienced by the tail rotor that is caused by the swirling motion of the main rotor wake.

The nonlinear simulation model developed is useful for the performance evaluation of linear feedback control systems, handling qualities research, and the design and evaluation of nonlinear feedback control systems. Feedback control laws that are based on the QS.M model and perform well in 6 D.O.F. QSM simulations are sometimes found to go unstable when implemented on the NRC Bell 205 helicopter. Previous work ${ }^{[1]}$ show that this is the result of rotor instability. The nonlinear RBM model can be used to quickly test control designs for rotor instability prior to flight test validation. 


\subsection{Recommendations}

Nifiough the body states of the RB.II model have been verified against flight test data. no - uch effort was made for the rotor or inflow states. one reason being the lack of data. During the course of this thesis. provisions were being made at NRC to install the required harduare and software to record the blade flapping angles. The validification of the rotor model wonld increase the usefulness of the model and would allow the tuning of the many rotor parameters which are presently approximated.

Further work can also be geared towards simulation of the engine governor. The RBMI model assumes that the engine governor is ideal and therefore the rotational speed remains constant. The dynamics of the engine governor can be expected to increase the lag in the ystem. For example a sudden increase in collective will cause a short term reduction in rotor speed which will affect the aerodynamic forces on the rotor and therefore delay the increase in lift.

The control actuators on the Bell 205 are used to translate pilot stick movement into blade feathering changes. The dynamics associated with these actuators are important for feedback control design and can be added to the RBM model as additional degrees of freedom. The control actuators will act to increase the lag in the system.

The main rotor sidewcsh at the tail rotor can be approximated with the flat-vortex wake theor: of reference [44] to extend the validity of the yaw model into the forward flight region. 
Appendix A

Trigonometric Identities 
The following trigonometric identities were used in the rotor equations of Chapter 2 to reduce all harmonics to the first order.

$$
\begin{aligned}
\sin ^{5} \psi & =\frac{10 \sin (\psi)-5 \sin (3 \psi)+\sin (5 \psi)}{16} \\
\cos ^{5} \psi & =\frac{10 \cos (\psi)+5 \cos (3 \psi)+\cos (5 \psi)}{16} \\
\sin ^{4} \psi \cos \psi & =\frac{2 \cos (\psi)-3 \cos (3 \psi)+\cos (5 \psi)}{16} \\
\cos ^{4} \psi \sin \psi & =\frac{2 \sin (\psi)+3 \sin (3 \psi)+\sin (5 \psi)}{16} \\
\sin ^{4} \psi & =\frac{3-4 \cos (2 \psi)+\cos (4 \psi)}{8} \\
\cos ^{4} \psi & =\frac{3+4 \cos (2 \psi)+\cos (4 \psi)}{8} \\
\sin ^{3} \psi \cos ^{3} \psi & =\frac{3 \sin (2 \psi)-\sin (6 \psi)}{32} \\
\sin ^{3} \psi \cos ^{2} \psi & =\frac{2 \sin (\psi)+\sin (3 \psi)-\sin (5 \psi)}{16} \\
\cos ^{3} \psi \sin ^{2} \psi & =\frac{2 \cos (\psi)-\cos (3 \psi)-\cos (5 \psi)}{16} \\
\sin ^{3} \psi \cos ^{2} \psi & =\frac{2 \sin (2 \psi)-\sin (4 \psi)}{8} \\
\cos ^{3} \psi \sin ^{2} \psi & =\frac{2 \sin (2 \psi)+\sin (4 \psi)}{8} \\
\sin ^{3} \psi & =\frac{3 \sin (\psi)-\sin (3 \psi)}{4} \\
\cos ^{3} \psi & =\frac{3 \cos (\psi)+\cos (3 \psi)}{4} \\
\sin ^{2} \psi \cos ^{2} \psi & =\frac{1-\cos (4 \psi)}{8} \\
\sin ^{2} \psi \cos ^{2} \psi & =\frac{\cos (\psi)-\cos (3 \psi)}{4} \\
\cos ^{2} \psi \sin ^{2} \psi & =\frac{\sin (\psi)+\sin (3 \psi)}{4} \\
\sin ^{2} \psi & =\frac{1-\cos (2 \psi)}{2} \\
\sin ^{2} \psi \cos ^{2} & =\frac{1+\cos (2 \psi)}{2} \\
2 & \frac{\sin (2 \psi)}{2} \\
& =1
\end{aligned}
$$




\section{Appendix B}

\section{NRC Bell 205 Flight Test Data}




\section{B.1 Hover Data}
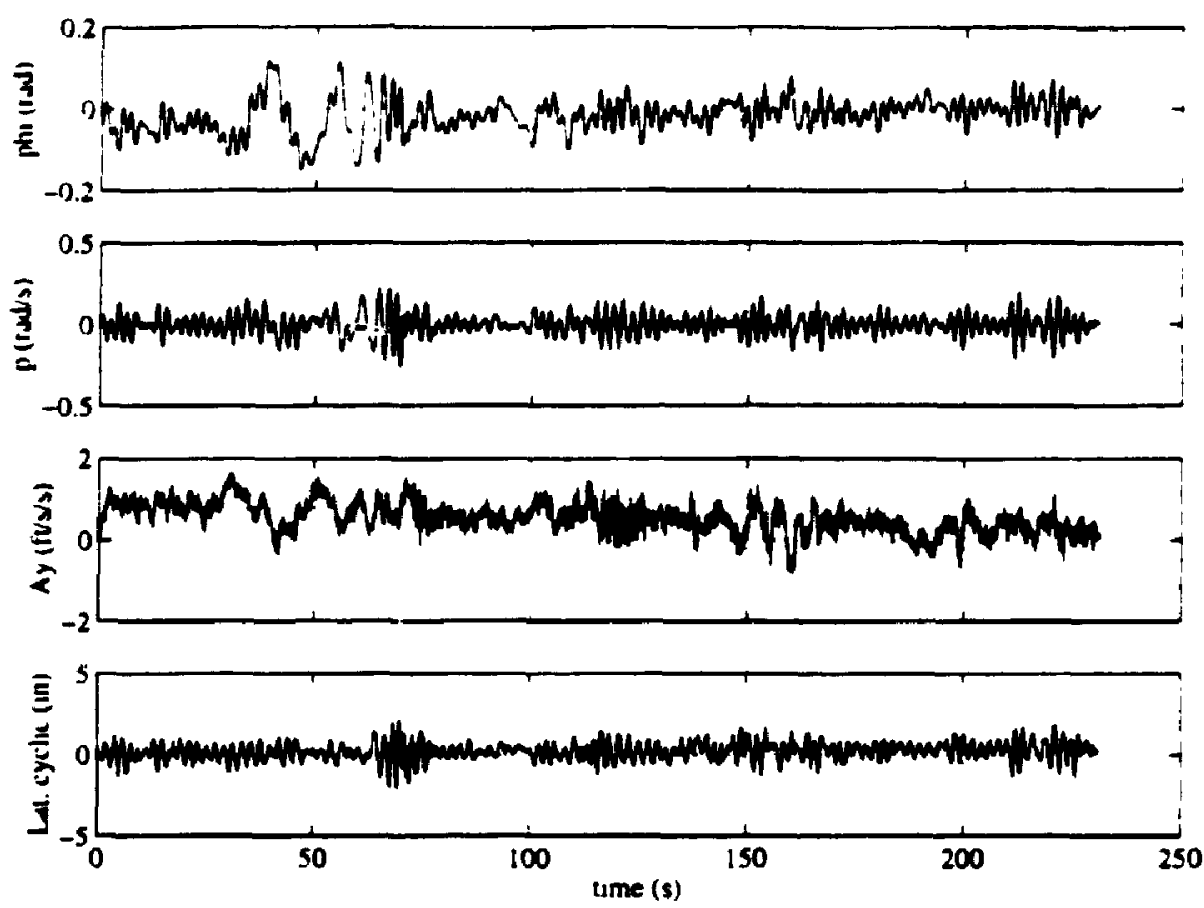

Figure B.1: Lateral channel frequency sweep
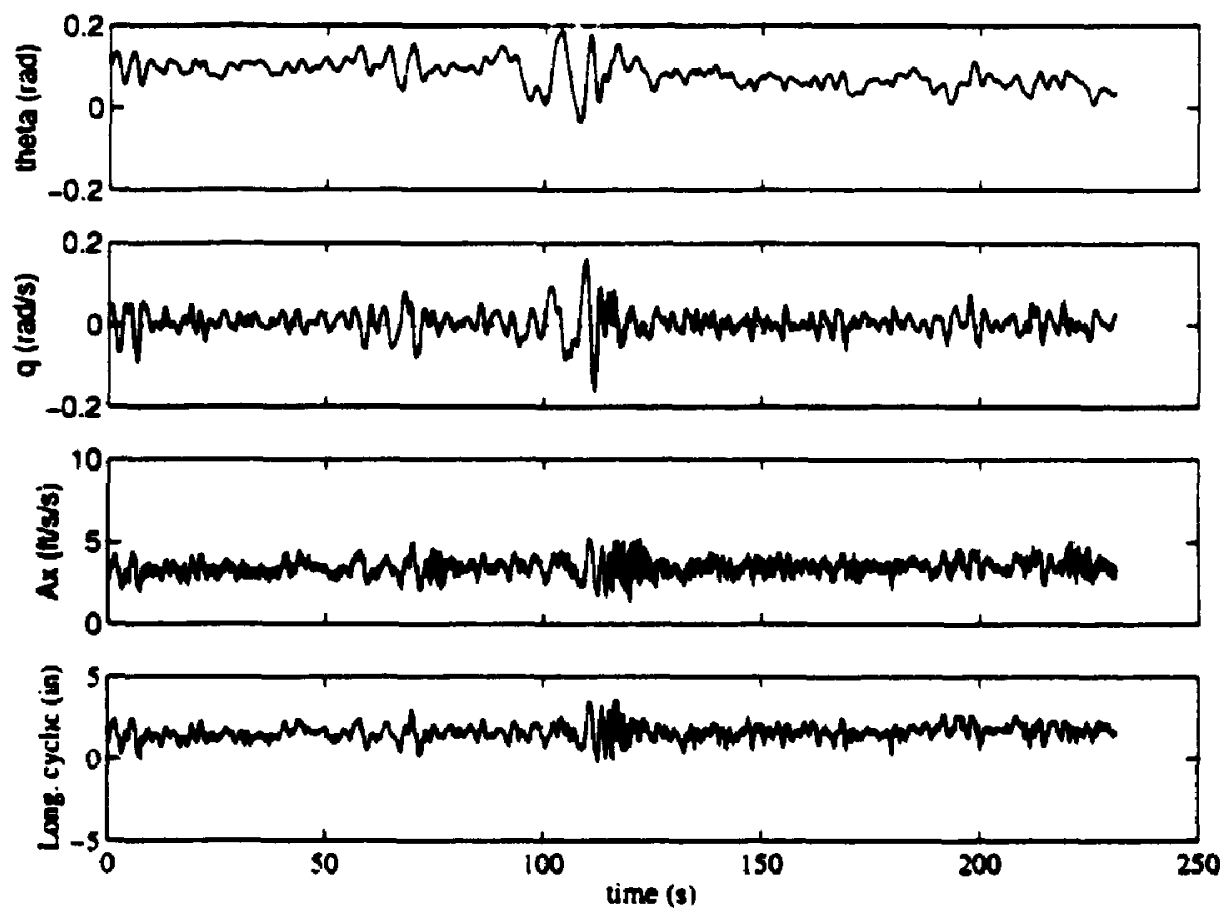

Figure B.2: Longitudinal channel frequency sweep 

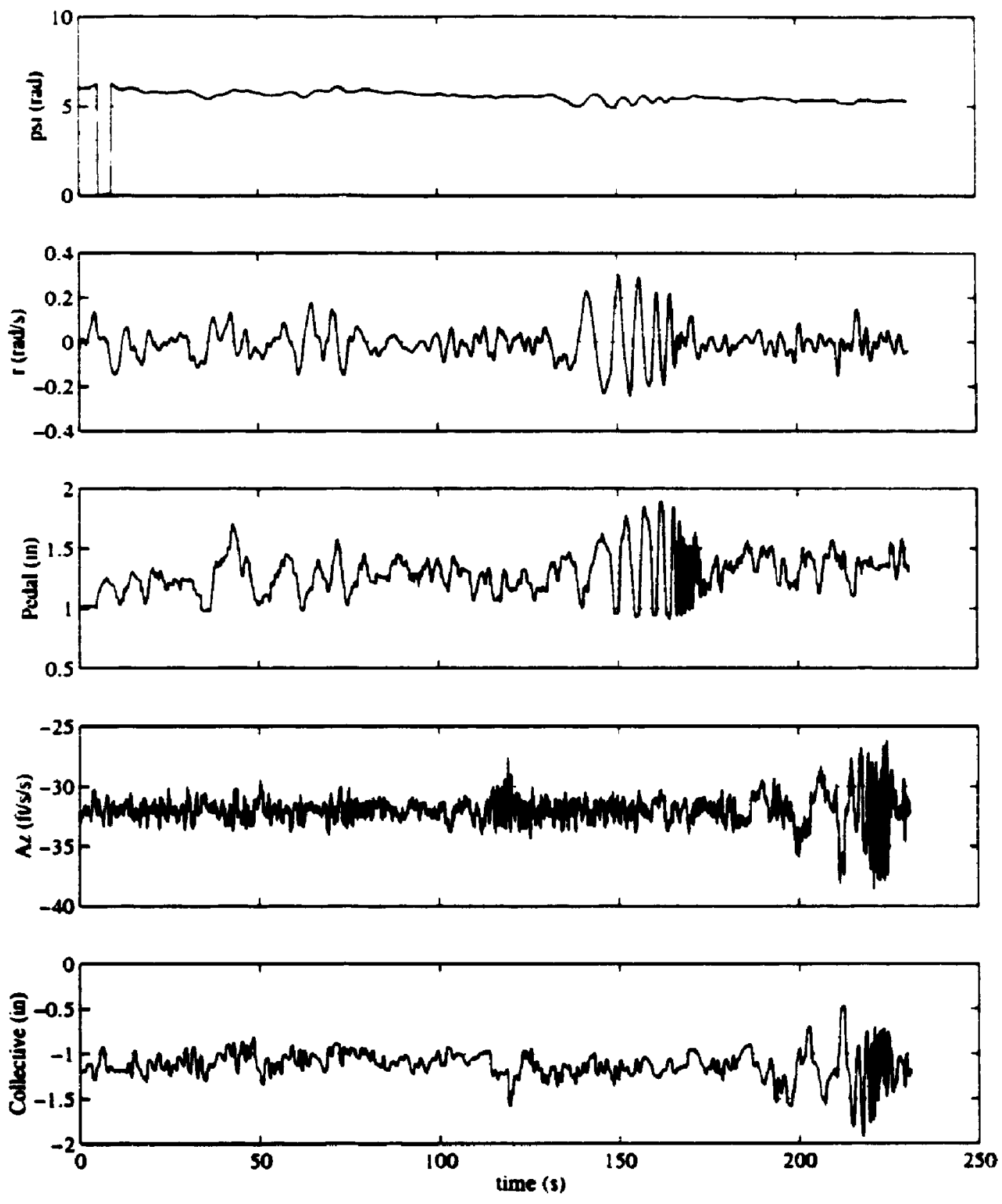

Figure B.3: Vertical and Yaw channel frequency sweeps 


\section{B.2 40 knots Data}
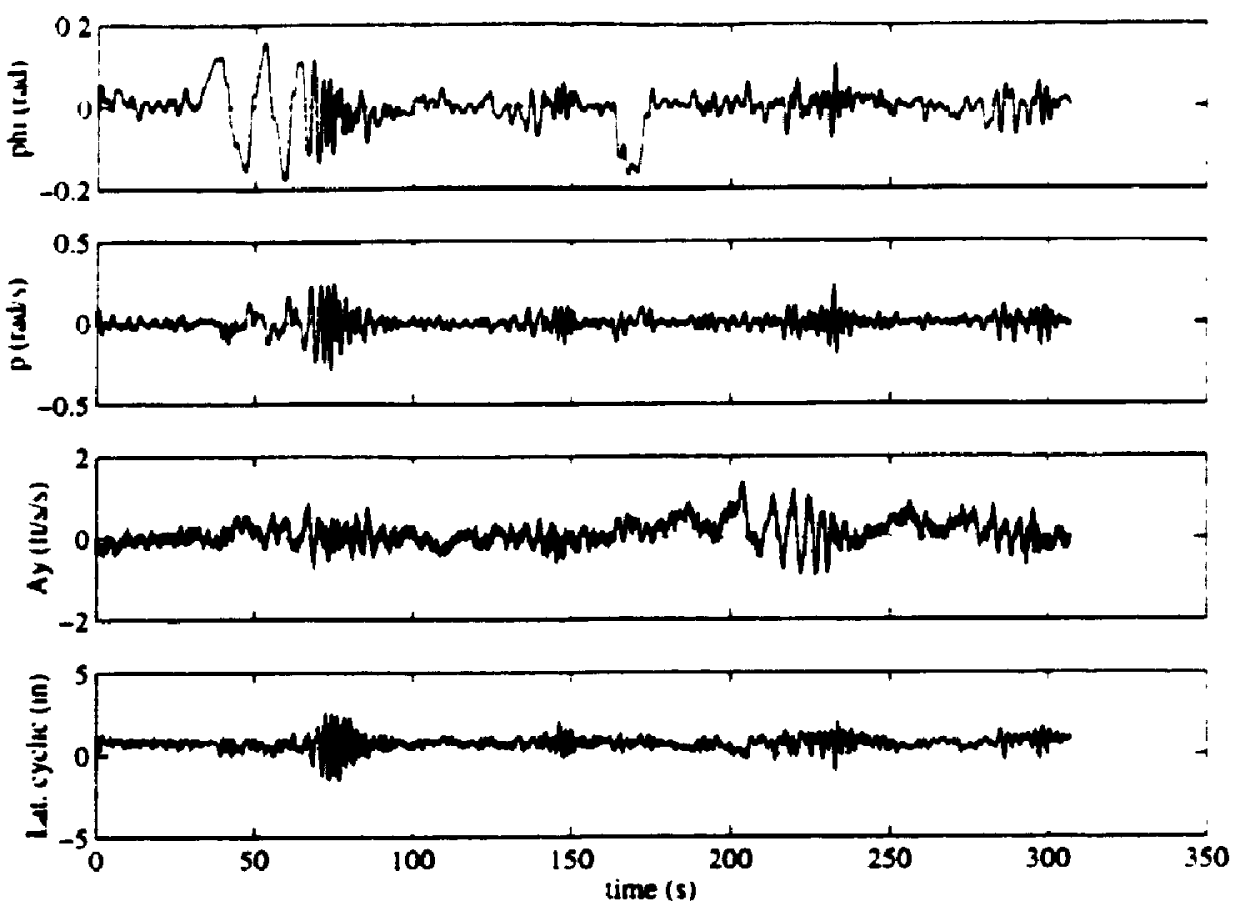

Figure B.4: Lateral channel frequency sweep
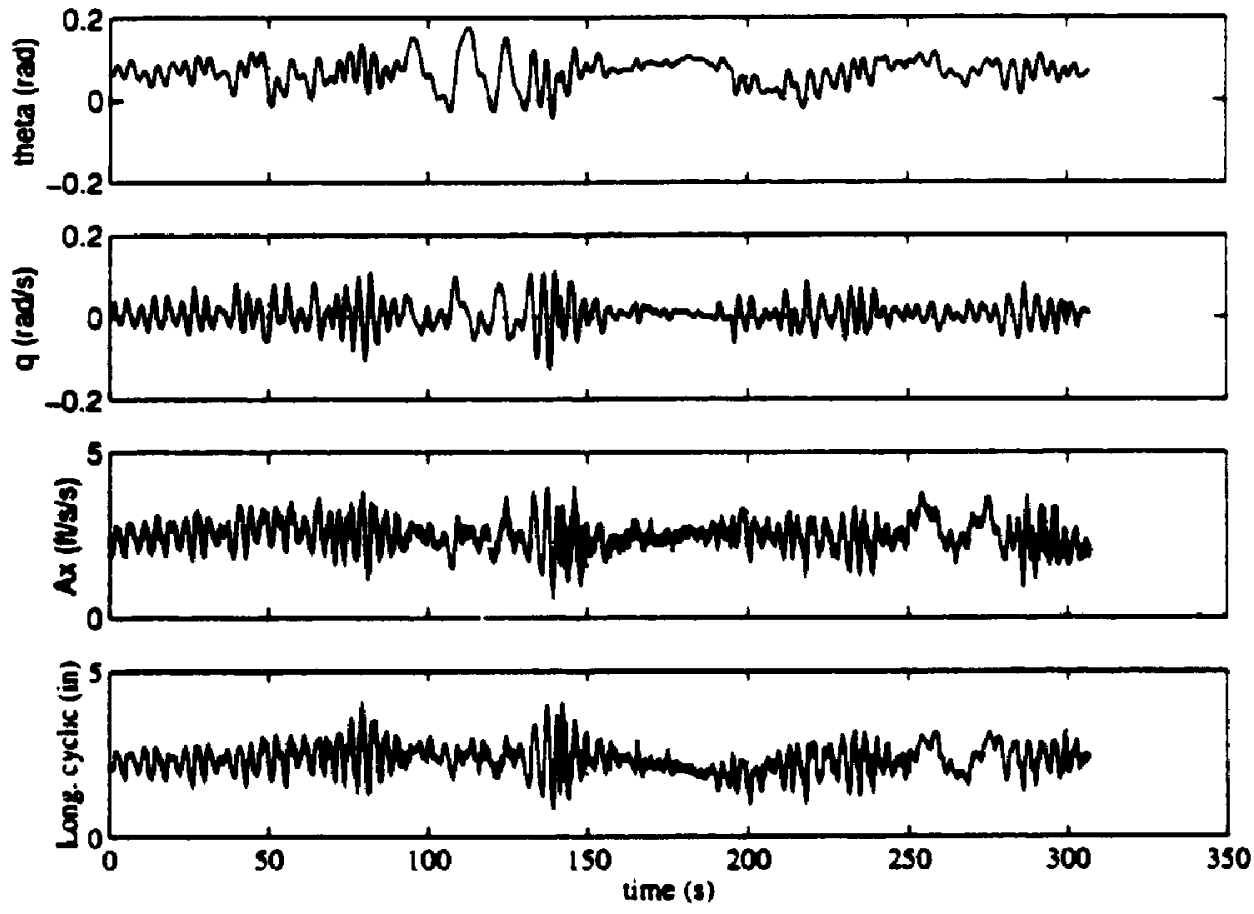

Figure B.5: Longitudinal channel frequency sweep 

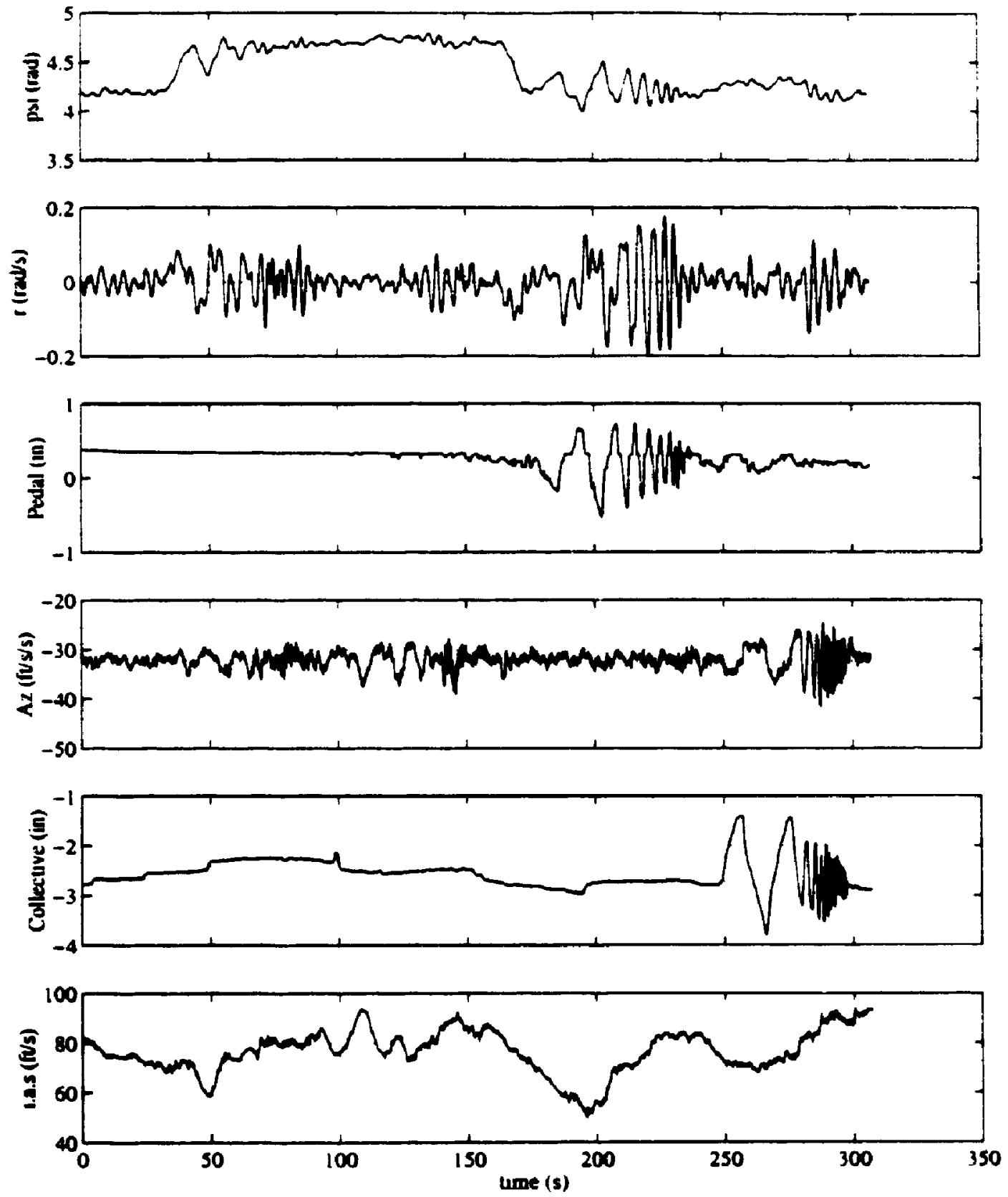

Figure B.6: Vertical and Yaw channel frequency sweeps 


\section{B.3 80 knots Data}
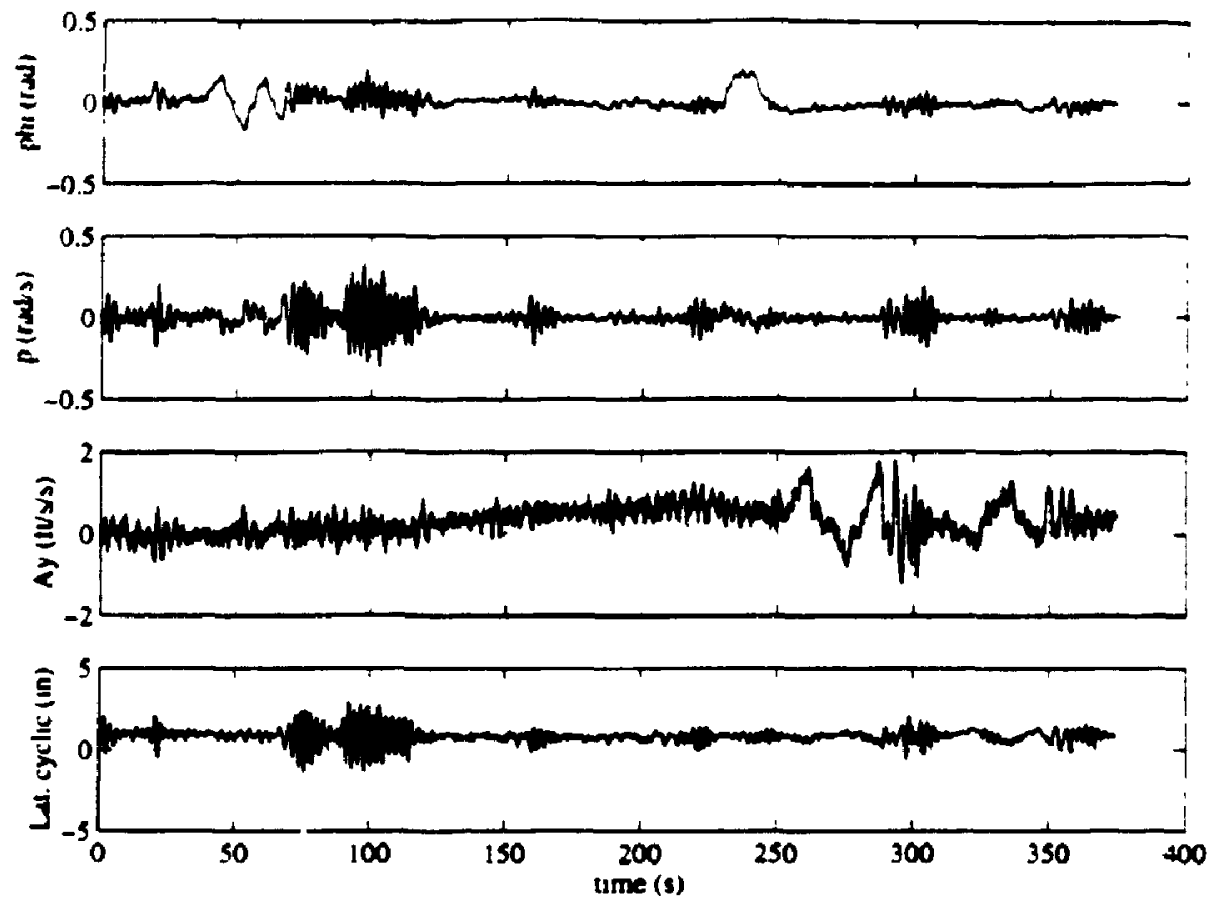

Figure B.7: Lateral channel frequency sweep
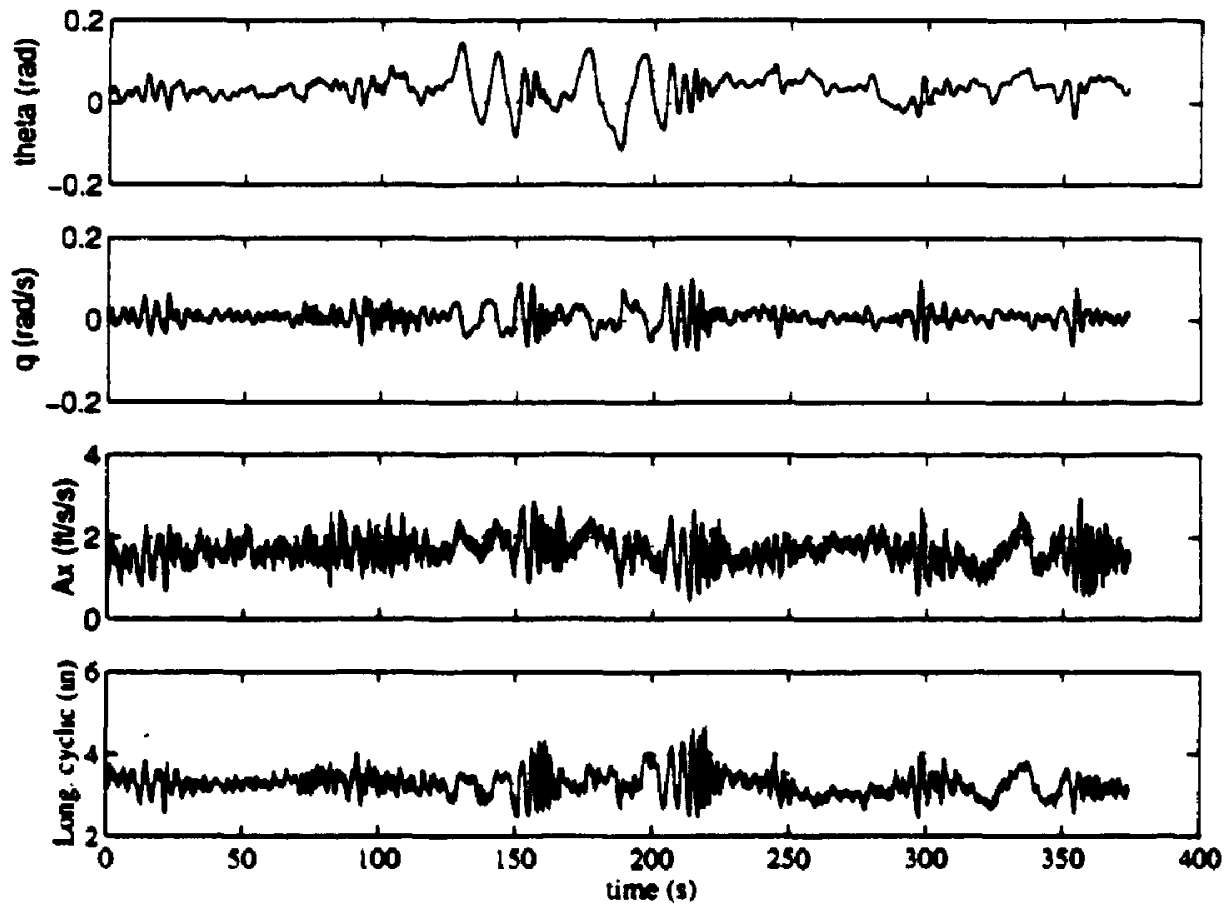

Figure B.8: Longitudinal channel frequency sweep 

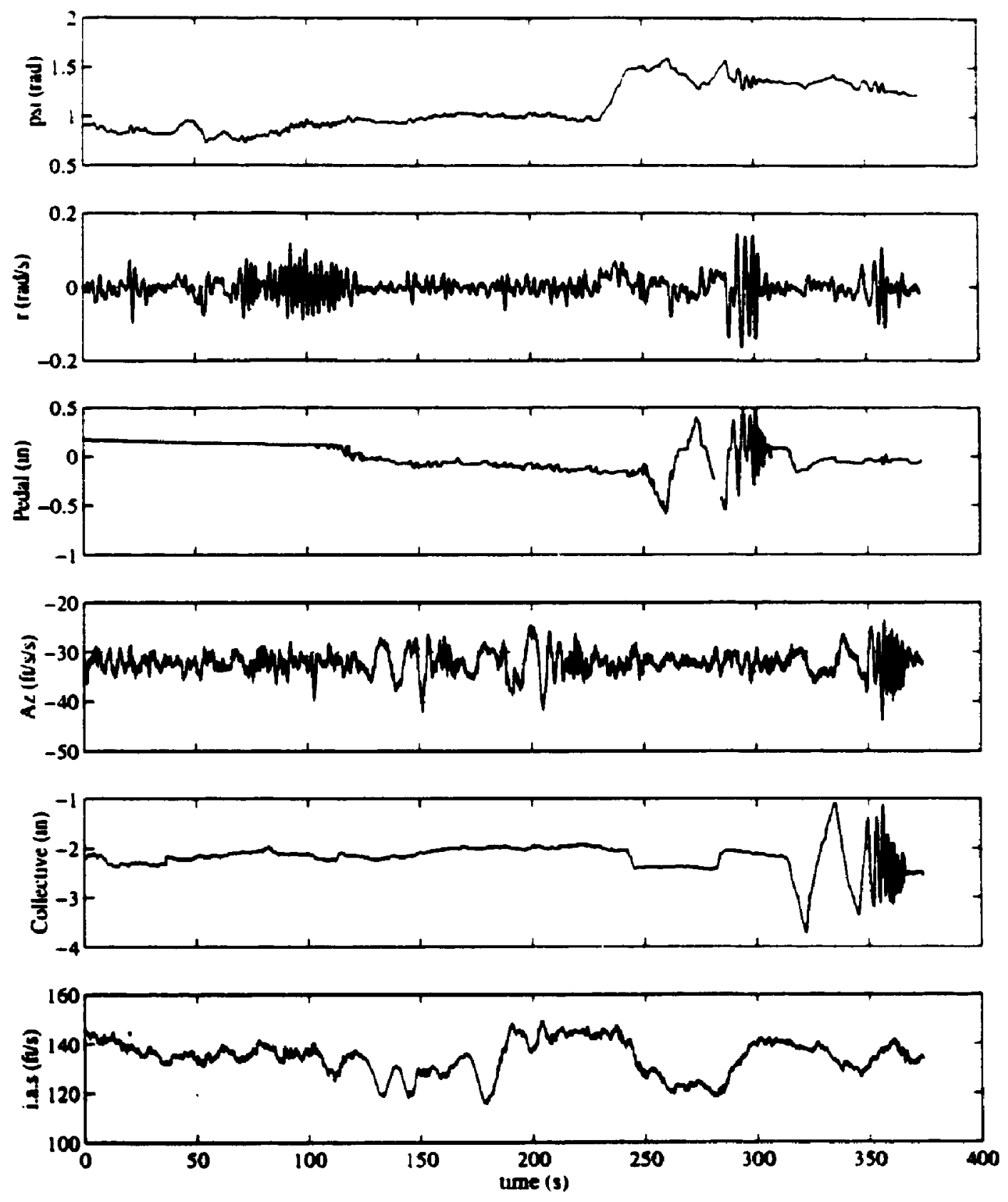

Figure B.9: Vertical and Yaw channel frequency sweeps 
Appendix C

Software Operating Manual 


\section{C.1 Compiling}

The program TRIMIMERPIT 4 is made up of two main files: 'Trimmerpit 4. for' and 'ForcesRD2.for'. The later is a collection of routines used to calculate the forces and angular accelerations of the main and tail rotors.

TRIMMERPIT 4 was written in standard Fortran $i-$ with the exception of extended source lines and extended continuation line limits. Inder the Sun Fortran compiler ${ }^{1}$ the following command can be used to compile the program:

677 -e -N1200 Trimmerpit4.for ForcesRD2.for

where -e allows extended source lines up to 132 characters and -N1200 allows up to 200 continuation lines. The routines in ForcesRD2.for are rarely changed and therefore it is beneficial to compile ForcesRD2.for separately and link later. In Sun Fortran:

$$
\text { fit - e -N1200 -c ForcesRD2.for }
$$

produces an object code file 'ForcesRD2.o' and later it can be linked:

$$
\text { fiT -e Trimmerpit4.for ForcesRD2.o }
$$

The file 'ForcesRD2.for' contains several include statements:

INCLUDE 'forces/HforceRD.for'

INCLUDE 'forces/YforceRD.for'

INCLUDE 'forces/ThrustRD.for'

INCLUDE 'forces/QtorqueRD.for'

INCLUDE 'forces/clmomRD.for'

INCLUDE 'forces/c2momRD.for'

INCLUDE 'forces/ddphetaRRD.for'

INCLUDE 'forces/ddphiRRD.for'

The file path above must be changed to conform to the path leading to the above files.

\footnotetext{
${ }^{1}$ Relesse 1.4
} 


\section{C.2 Program Main Menu}

The main menu of the program is shown bellow followed by a description of each option.

(1) FIND A,B MATRICES (about some trim point)

(2) RUN SIMULATION USING CONTROL

(3) FIND TRIM POINT

(4) RUN SIMULATION USING NO CONTROL

(5) QUIT

\section{C.2.1 (1) FIND A,B MATRICES (about some trim point)}

This option is used to calculate the linear model about some trim point. The matrices $A$ and $b$ are the state space matrices corresponding to:

$$
\dot{\boldsymbol{x}}=A \boldsymbol{x}+\boldsymbol{B} \boldsymbol{u}
$$

The routine will prompt the user for the file containing the trim states, TRIMFILE, and the file containing the state perturbations, PRTBFILE, which are to be used in deriving the linear model.

PRTBFILE must be an ASCII file containing eight numbers, one on each line:

1. number of iterations performed to find $\mathrm{dx} / \mathrm{dt}$

2. velocity perturbation $(\mathrm{m} / \mathrm{s})$

3. body angle perturbation (rad)

4. body angular rate perturbation ( $\mathrm{rad} / \mathrm{s})$

5. rotor angle perturbation (rad)

6. rotor angular rate perturbation ( $\mathrm{rad} / \mathrm{s})$

7. control perturbation (rad)

8. downwash perturbation $((\mathrm{m} / \mathrm{s}) /(\mathrm{m} / \mathrm{s}))$

9. tail rotor induce velocity perturbation $(\mathrm{m} / \mathrm{s})$ 
10. stabilizer bar state perturbation (rad)

The full $A$ and $B$ matrices are first derived and stored in ASTB and BST B. The state numbers that are included in ASLB and BSCB are contained in the data artay PS C.ACF. The state numbers contained in PSTATE correspond to the computer simulation itdte used throughout the program and are described in the header of the program. The 1 and $B$ matrices that are passed back to the main routines are a partitioned version of ASCB and BSUB. They are partitioned according to the values stored in the data array RSTATE. i.e. a value of 3 in RSTATE corresponds to the third row and column (or wtate) of ASCB and the third row (or state) of BSCB.

The $A$ and $B$ matrices are written, column-wise, to a the file 'Matrix.dat' (first $A$ id $d$ then $B$ ).

\section{C.2.2 (2) RUN SIMULATION U'SING CONTROL}

This option is used to run a simulation 1:sing the controller found in subroutine ODEI.VT. The user is asked for three inputs. First, the file name containing the trim states, serond. the file that contains the feedback gains required to stabilize the helicopter ${ }^{2}$, and third. the name of the output file where the results are to be stored.

For the present controller, the trim file must contain the following data:

- trim values of NSTATE(currently 27) states (one per line)

- trim values for control states, one per line

- TMR main rotor thrust at trim

- TTR tail rotor thrust at trim

- LAMDAM non-dimensional induced velocity (see routine DYNINFLOW)

- CFERR longitudinal velocity integral error

- VFERR lateral velocity integral error

- WFERR vertical velocity integral error

\footnotetext{
${ }^{2}$ see section C. 4
} 
- start time for simulation

- end time for simulation

\section{C.2.3 (3) FIND TRIM POINT}

This option finds the trim point of the helicopter by 'flying' to the trim point. This is usually achieved by starting at hover and commanding a certain longitudinal or lateral velocity. The simulation is run until all oscillation in the states are negligible and then the states are recorded as the trim states.

At this point in time this routine is rather crude and the user must manually change the time required to reach trim (about 15 to 20 seconds past the time the helicopter first. reaches the new set-point).

Future work should augment this routine with a linear regression algorithm which forces the helicopter state derivatives to zero by manipulating the controıs.

\section{C.2.4 (4) RUN SIMULATION USING NO CONTROL}

This option is identical to option (2) but now the control is held fixed at the trim values and the resulting motion of the helicopter recorded.

\section{C.3 Integration of States}

A Runga-Kutta method with adaptive step size control and truncation error correction is used to propagate the states with time. The driver routine is ODEINT which calls RKt and RKQC. All routines were taken from Press ${ }^{[36]}$ and modified.

The following are the integration parameters used in the main program:

KMAX - the maximum number of steps that can be stored

DXSAV-results are only stored at intervals of time greater than DXSAV

H.MIN- the minimurn allowed step size

H.MAX- the maximum allowed step size

EPS- a factor used to set accuracy of iesult

lote: The routines used to calculate the forces on tue helicopter (MAINFRC, TAILFRC, E.MPE.YAGE, FSLAGE) are only called once per time step to reduce computation time. 
They should be called from within the DERIVS routine rather than ODEINT if time is not a factor. However. the difference between the two methods was found to be insignificant for high accuracy (low EPS).

Dynamic error analysis[3i] shows that a step size of 0.0045 seconds or less is required to capture the correct dynamics of the rotor flapping frequencies. However this step size produces a 'noisy' signal in the rotor state derivatives and it is necessary to reduce the maximum step size to 0.001 seconds. This problem is likely due to instabilities in the integration routine which are common in helicopter simulation ${ }^{[46]}$.

\section{C.4 Applying Control}

Control is applied using the following code which can be found in the subroutine ODEINT:

$\mathrm{C}$

C - APPLY CONTROL IF THE REQUIRED TIME HAS ELAPSED

$\mathrm{C}$

CHECK $=\mathrm{X}-\mathrm{XOLD}$

IF ((CHECK .GE. TCONT) .AND. (CONTROL .EQ. .TRCE.)) THE.

WFERR $=0.0 \mathrm{DO}-\mathrm{Y}(12)$

VFERR $=$ VFERR $+\mathrm{Y}(11) \cdot 0.0 \mathrm{DO}$

LFERR $=$ UFERR $+\mathrm{Y}(10) \cdot 0.0 \mathrm{D} 0$

$\mathrm{C.V}(1)=\mathrm{CN}(1)+-1.0 \mathrm{D} 0^{*} \mathrm{KO}(1)^{*} \mathrm{WFERR}+\mathrm{KO}(2)^{*} \mathrm{DYDX}(12)$

LATSTK $=\mathrm{KC}(5)^{*} \mathrm{Y}(5)+\mathrm{KC}(6)^{*} \mathrm{Y}(6)+\mathrm{KC}(11)^{*} \mathrm{Y}(11)+\mathrm{IKC}(1)^{*} \mathrm{VFERR}$

LONSTK $=\mathrm{KS}(7)^{*} \mathrm{Y}(7)+\mathrm{KS}(8)^{*} \mathrm{Y}(8)+\mathrm{KS}(10)^{*} \mathrm{Y}(10)+\mathrm{IKS}(1)^{*} \mathrm{UFERR}$

$\mathrm{CN}(4)=\mathrm{CN}(4)+\operatorname{KOTR}(1)^{*} Y(9)+\operatorname{KOTR}(2)^{*} \mathrm{DYDX}(9)+0.1 \mathrm{D0} 0^{*}(20)$

$\mathrm{XOLD}=\mathrm{X}$

END IF

$\mathrm{C}$

C - CHANGE LONG. AND LAT. 'STICK' DEFLECTIONS ABOVE TO ACCOU.NT

C - FOR STABILIZER P.AR EFFECT IF THE STABILIZER IS ON

$\mathrm{C}$

IF (SBAR .EQ. YES) THEN

$\mathrm{CN}(2)=$ LONGSTK $\cdot Y(26)$ 


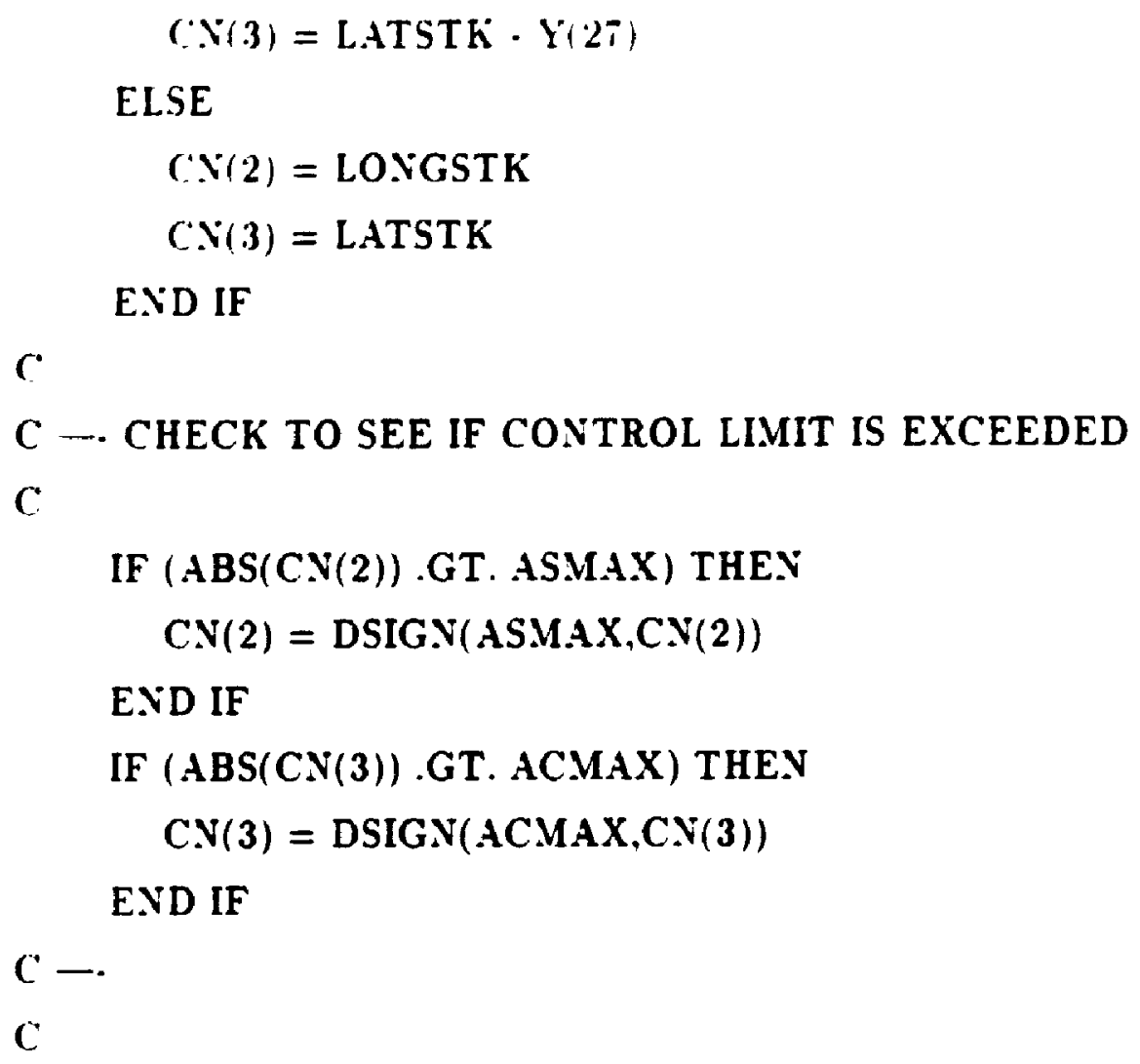

where.

$$
\begin{aligned}
\text { CN }= & \text { Control vector. } \\
\text { LONSTK,LATSTK = } & \text { Longitudinal and lateral cyclic respectively (before } \\
& \text { alteration due to stabilizer bar) } \\
Y= & \text { State vector. } \\
\mathrm{KC}= & \text { Feedback gain vector for lateral channel. } \\
\mathrm{KS}= & \text { Feedback gain vector for longitudinal channel. } \\
\mathrm{X}= & \text { Time. } \\
\text { XOLD = } & \text { Time at which control was last applied. } \\
\text { ASMAX ICMAX = } & \text { Physical limits on the longitudinal and lateral cyclic }
\end{aligned}
$$

If it is desired to command a $2 \mathrm{~m} / \mathrm{s}$ forward velocity then the following line should be exchanged with its counterpart above:

$$
\text { UFERR }=\text { UFERR }+Y(10)-2.0 D 0
$$


Control is only applied if the time elapsed since the last application of control. ( $H E(' K$. is greater than TCONT (currently $0.01 \mathrm{sec}$ ). Therefore control is only applied at discrete intervals of 0.01 seconds or greater. This was found to be tuecessary berause the collective( $\mathrm{C} . \mathrm{I}(1))$ and peddle $(\mathrm{CX}(4))$ control regimes are additive and tend to cause overcontrol ' when the adaptive integration routine takes rather small steps.

The longitudinal( $\mathrm{CV}(2))$ and lateral (C.V(3)) use a form of proportional derivative (PD) control on attitude and proportional-integral(PI) control on velocity. The integral errors are LFERR and VFERR respectively. The feedback gains are (found in file ciontrol.dat):

\begin{tabular}{|c|c|}
\hline $\mathrm{KC}(5)$ & $=5.5$ \\
\hline $\mathrm{KC}(6)$ & $=0.32$ \\
\hline $\mathrm{KC}(11)$ & $=3.0$ \\
\hline $\operatorname{IKC}(1)$ & $=0.05$ \\
\hline $\mathrm{KS}(7)$ & $=-10.0$ \\
\hline $\mathrm{KS}(8)$ & $=-0.32$ \\
\hline $\mathrm{KS}(10)$ & $=1.5$ \\
\hline $\operatorname{IKS}(1)$ & $=0.015$ \\
\hline $\mathrm{KO}(1)$ & $=0.05$ \\
\hline $\mathrm{KO}(2)$ & $=0.001$ \\
\hline KOTR(1) & $=0.05$ \\
\hline KOTR(2) & $=0.025$ \\
\hline
\end{tabular}

These gains tend to produce a rather tight controller and is not suitable for some applications. The gains used on the Bell 205 at the NRC are much lower and produce a slower response on the simulation. The NRC gains for lateral control are:

$$
\begin{aligned}
\mathrm{KC}(5) & =15-20 \mathrm{in} / \mathrm{rad}=0.4-0.53 \mathrm{rad} / \mathrm{rad} \\
\mathrm{KC}(6) & =15-21 \mathrm{in} /(\mathrm{rad} / \mathrm{sec})=0.4-0.56 \mathrm{rad} /(\mathrm{rad} / \mathrm{s}) \\
\mathrm{KC}(11) & =0.016 \mathrm{rad}(\text { of attitude }) /(\mathrm{ft} / \mathrm{s})=0.052 \mathrm{rad}(\text { of att. }) /(\mathrm{m} / \mathrm{s})
\end{aligned}
$$

where $\mathrm{KC}(11)$ is the feedback gain on velocity used to get a commanded roll attitude.

Commanding a sudden velocity increase greater than $8 \mathrm{~m} / \mathrm{s}$ ( $16 \mathrm{knots}$ ) tends to cause problems in the simulation, mainly in the dynamic inflow routine and control instability. Figure $C .1$ shows a sudden forward velocity command of $8 \mathrm{~m} / \mathrm{s}$ and the resulting saturation 

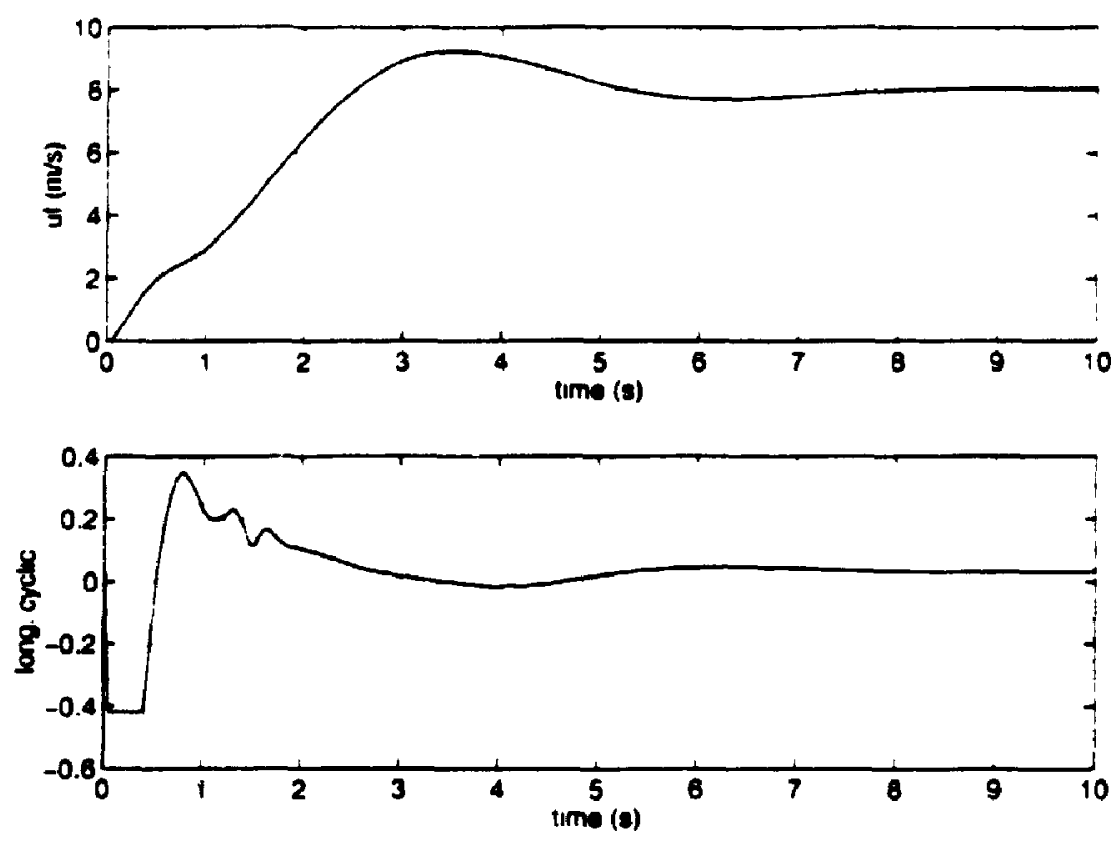

Figure C.1: Sudden forward velocity command of $8 \mathrm{~m} / \mathrm{s}$ from hover

of longitudinal cyclic. Control saturation and dynamic inflow problems are avoided by using a ramp speed command as follows:

IF ((X .GE. 0.0D0) .AND. (X .LT. 30.0D0)) THEN

UFERR $=$ UFERR + Y(10) $-1.0 \mathrm{DO} 0(\mathrm{X}-10.0 \mathrm{D} 0)$

ELSE IF ( $X$.GE. 40.0D0) THEN

UFERR $=$ UFERR $+Y(10) \cdot 30.0 D 0$

\section{ELSE}

UFERR $=$ UFERR $+\mathrm{Y}(10) \cdot 0.0 \mathrm{D} 0$

\section{END IF}

The above commands a linear speed increase from hover to $30 \mathrm{~m} / \mathrm{s}$ ( $58 \mathrm{knots}$ ) in 30 seconds, thus avoiding any instantaneous jerks in control. 


\section{Appendix D}

\section{Helicopter Parameters ${ }^{[3],[46-48]}$}




\begin{tabular}{|c|c|c|c|}
\hline Name & Symbol & Cinits & Value \\
\hline \multicolumn{4}{|c|}{ Helicopter inertia and mass (nominal) } \\
\hline Mass & $m$ & $\mathrm{~kg}$ & 3629 \\
\hline Pitch inertia & $I_{y y}$ & $\mathrm{~kg} \cdot \mathrm{m}^{2}$ & $1+684$ \\
\hline Roll inertia & $I_{x x}$ & $\mathrm{~kg} \cdot \mathrm{m}^{2}$ & 3966 \\
\hline Yaw inertia & $I_{z z}$ & $\mathrm{~kg} \cdot \mathrm{m}^{2}$ & 12541 \\
\hline Cross product of inertia & $I_{x z}$ & $\mathrm{~kg} \cdot \mathrm{m}^{2}$ & 1695 \\
\hline Center of gravity stationline & & in & $13 i$ \\
\hline Center of gravity buttline & & in & 0 \\
\hline Center of gravity waterline & & in & .57 .5 \\
\hline \multicolumn{4}{|l|}{ Lain rotor } \\
\hline Number of blades & $b$ & $\mathbf{N} / \mathbf{A}$ & 2 \\
\hline Hub type & & $\mathrm{N} / \mathrm{A}$ & Teetering \\
\hline Radius & $R$ & m & $i .3152$ \\
\hline Blade root cutout factor & $x_{0}$ & $\mathrm{~N} / \mathrm{A}$ & 0.0833 \\
\hline Chord & $c$ & $\mathrm{~m}$ & 0.5334 \\
\hline Section & & $\mathrm{N} / \mathrm{A}$ & VACA 0012 \\
\hline Lift curve slope & $a$ & $\mathrm{rad}^{-1}$ & 6.3 \\
\hline Undersling & $Z_{S H}$ & $\mathrm{~m}$ & 0.132 \\
\hline Blade twist & $\theta_{t}$ & $\mathrm{rad}$ & -0.19024 \\
\hline Longitudinal Shaft tilt & $i_{m}$ & $\mathrm{rad}$ & 0.0872665 \\
\hline Rotational speed & $\Omega$ & $\mathrm{rad} / \mathrm{s}$ & 33.9292 \\
\hline Flapping inertia ${ }^{\dagger}$ & $I_{y y}$ & $\mathrm{~kg} \cdot \mathrm{m}^{2}$ & 3292 \\
\hline Rotational inertia & $I_{z z}$ & $\mathrm{~kg} \cdot \mathrm{m}^{2}$ & 3304 \\
\hline Feathering inertia & $I_{x x}$ & $\mathrm{~kg} \cdot \mathrm{m}^{2}$ & 12 \\
\hline Flapping spring constant & & $\mathrm{N}-\mathrm{m} / \mathrm{rad}$ & 0 \\
\hline Pitch flap coupling & & $\mathrm{N} / \mathrm{A}$ & 0 \\
\hline Precone angle & $c_{0}$ & $\mathrm{rad}$ & 0.047124 \\
\hline Drag coefficient 0 & $c_{d_{0}}$ & $\mathrm{~N} / \mathrm{A}$ & 0.0084 \\
\hline Drag coefficient 1 & $c_{d_{1}}$ & $\mathrm{~N} / \mathrm{A}$ & 0 \\
\hline Drag coefficient 2 & $c_{d_{2}}$ & $\mathrm{~N} / \mathrm{A}$ & 0.36 \\
\hline Hub stationline & & in & 133.048 \\
\hline Hub waterline & & in & 141.844 \\
\hline
\end{tabular}




\begin{tabular}{|c|c|c|c|}
\hline Same & Symbol & Inits & Value \\
\hline \multicolumn{4}{|l|}{ Tail rotor } \\
\hline Number of blades & $b_{t n}$ & $\therefore / A$ & 2 \\
\hline Hub type & & $\mathrm{N} / \mathrm{A}$ & Teetering \\
\hline Radius & $R_{T R}$ & $\mathbf{m}$ & 1.2954 \\
\hline Blade root cutout factor & $x_{q_{T R}}$ & $N / A$ & $0.088: 2$ \\
\hline Chord & $c_{T R}$ & $\mathrm{~m}$ & 0.286 \\
\hline Lift curve slope & $a_{R R}$ & $\mathrm{rad}^{-1}$ & 6.6 \\
\hline Blade twist & $\theta_{t_{T R}}$ & rad & 0 \\
\hline Rotational speed & $\Omega_{T R}$ & $\mathrm{rad} / \mathrm{s}$ & $5.123 \Omega$ \\
\hline Flapping inertia ${ }^{\dagger}$ & $I_{y y_{T R}}$ & $\mathrm{~kg}-\mathrm{m}^{2}$ & 2.1094 \\
\hline Flapping spring constant & & $\mathrm{N}-\mathrm{m} / \mathrm{rad}$ & 0 \\
\hline Pitch flap coupling & & $\mathrm{N} / \mathrm{A}$ & $\mathbf{0}$ \\
\hline Precone angle & $c_{q_{T R}}$ & rad & 0 \\
\hline Drag coefficient 0 & $c_{d_{T R}}$ & $\mathrm{~N} / \mathrm{A}$ & 0.0084 \\
\hline Drag coefficient 1 & $c_{d_{1_{T R} R}}$ & $\mathrm{~N} / \mathrm{A}$ & $\mathbf{0}$ \\
\hline Drag coefficient 2 & $c_{d_{Z_{T R}}}$ & $\mathrm{~N} / \mathrm{A}$ & 0.36 \\
\hline Hub stationline & & in & 479.4 \\
\hline Hub waterline & & in & 137.6 \\
\hline Hub buttline & & in & -15.16 \\
\hline \multicolumn{4}{|l|}{ Horizontal stabilizer } \\
\hline Maximum lift coefficient & $\left(C_{L_{m}}\right)_{H S}$ & $\mathrm{~N} / \mathrm{A}$ & 1.2 \\
\hline Aspect ratio & $A \cdot R_{H S}$ & $\mathrm{~N} / \mathrm{A}$ & 2.009 \\
\hline Lift curve slope at zero sideslip & $a_{a_{H S}}$ & $\mathrm{rad}^{-1}$ & 3.9 .53 \\
\hline Planform area & $S_{H S}$ & $\mathrm{~m}^{2}$ & 1.016 \\
\hline Incidence angle & $i_{4} s$ & rad & variable \\
\hline Dynamic pressure ratio & $\eta_{H S}$ & $\mathrm{~N} / \mathrm{A}$ & 0.8 \\
\hline Center of pressure stationline & & in & 363.0 \\
\hline Center of pressure waterline & & in & 64.83 \\
\hline Center of pressure buttline & & in & \pm 28.1 \\
\hline
\end{tabular}

t all inerwas for complete rotor-hub cuembly 


\begin{tabular}{|c|c|c|c|}
\hline Vame & Symbol & Inits & Value \\
\hline \multicolumn{4}{|l|}{ lertical fin } \\
\hline Maximum lift coefficinnt & $\left(C_{L m}\right)_{i F}$ & $\therefore / A$ & 1.2 \\
\hline Aspect ratio & $A \cdot R_{V_{F}}$ & $X / A$ & 1.426 \\
\hline Lift curve slope at zero sideslip & $a_{q_{r},}$ & $\mathrm{rad}^{\cdot:}$ & 2.615 \\
\hline Planform area & $S_{V \cdot F}$ & $\mathrm{~m}^{2}$ & 1.0359 \\
\hline Incidence angle & $i_{F}$ & $\mathrm{rad}$ & 0 \\
\hline Dynamic pressure ratio & $\eta_{F}$ & $\mathrm{~N} / \mathrm{A}$ & 0.9 \\
\hline Center of pressure stationline & & in & 450.3 \\
\hline Center of pressure waterline & & in & 104.7 \\
\hline Center of pressure buttline & & in & 0.4 \\
\hline \multicolumn{4}{|l|}{ Fuseloge } \\
\hline Aerodynamic Reference stationline & & in & 137 \\
\hline Aerodynamic Reference stationline & & in & 57.5 \\
\hline Aerodynamic Reference stationline & & in & .57 .5 \\
\hline Drag, $\alpha=3=0^{\circ}$ & $\frac{D_{0}}{q}$ & $\mathrm{~m}^{2}$ & 0.659612 \\
\hline Drag variation with $\alpha$ & $\frac{\partial D / q}{\partial \alpha}$ & $\mathrm{m}^{2} / \mathrm{rad}$ & 0.789676 \\
\hline Drag variation with $\alpha^{2}$ & $\frac{\partial^{2} D / \Omega}{\partial \alpha^{2}}$ & $\mathrm{~m}^{2} / \mathrm{rad}^{2}$ & 14.492874 \\
\hline Drag variation with $3^{2}$ & $\frac{\partial^{2} D / q}{\partial s^{2}}$ & $\mathrm{~m}^{2} / \mathrm{rad}^{2}$ & 0 \\
\hline Drag at $\alpha=90^{\circ}$ & $\left.\frac{D}{q}\right|_{a=9}$ & $\mathrm{~m}^{2}$ & 8.361274 \\
\hline Drag at $\beta=90^{\circ}$ & $\left.\frac{D}{Q}\right|_{B=90^{\circ}}$ & $\mathrm{m}^{2}$ & 3.901928 \\
\hline Lift, $\alpha=3=0^{\circ}$ & $\frac{L_{0}}{q}$ & $\mathrm{~m}^{2}$ & 0.185806 \\
\hline Lift variation with $\alpha$ & $\frac{\partial L / q}{\partial \alpha}$ & $\mathrm{m}^{2} / \mathrm{rad}$ & 0.3241606 \\
\hline Sideforce variation with $\beta$ & $\frac{\partial Y / q}{\partial \beta}$ & $\mathrm{m}^{2} / \mathrm{rad}$ & 1.0962559 \\
\hline Rolling moment with $\beta$ & $\frac{\partial l / g}{\partial s}$ & $\mathrm{~m}^{3} / \mathrm{rad}$ & .3 .9643585 \\
\hline Rolling moment at $\beta=90^{\circ}$ & $\left.\frac{l}{3}\right|_{\beta=90^{\circ}}$ & $\mathrm{m}^{3}$ & 0 \\
\hline Pitch moment, $\alpha=\beta=0^{\circ}$ & $\frac{M_{0}}{q}$ & $\mathrm{~m}^{3}$ & 0.1953862 \\
\hline Pitch moment variation with $\alpha$ & $\frac{\partial M / Q}{\partial \alpha}$ & $\mathrm{m}^{3} / \mathrm{rad}$ & 8.6932719 \\
\hline Pitch moment at $\alpha=90^{\circ}$ & $\left.\frac{M}{9}\right|_{\alpha=900}$ & $\mathrm{~m}^{3}$ & 16.990108 \\
\hline Yaw moment variation with $\beta$ & $\frac{\partial N / q}{\partial s}$ & $\mathrm{~m}^{3} / \mathrm{rad}$ & .0 .1982179 \\
\hline Yaw moment at $\beta=90^{\circ}$ & $\left.\frac{v}{Q}\right|_{\beta=90^{\circ}}$ & $\mathrm{m}^{3}$ & $\cdot 11.3267386$ \\
\hline
\end{tabular}


Appendix E

Linear Model Parameters (Part I) 
The following tables define the $A$ and $B$ state space matrices of the linear RBM model used in chapter 4 . The left most column in each table contains the state derivative vector and the upper row in each table contains the state vector. The given parameters are in standard SI units so that the body and rotor state elements in the state vector have units of rad, $\mathrm{rad} / \mathrm{s}$, and $\mathrm{m} / \mathrm{s}$. The main rotor inflow states, $\left(\lambda_{0}, \lambda_{\theta}, \lambda_{c}\right)$, are dimensionless and the tail rotor inflow state, $v_{i_{\mathrm{r} n}}$, is in $\mathrm{m} / \mathrm{s}$. All the elements of the control vector $\left(\theta_{g_{R}}, A_{s}, A_{c}, \theta_{q_{R}}\right)$ have units of radians. The units of the trim states presented comply to the above except that all angular valtes are in $\mathrm{deg}$ and $\mathrm{deg} / \mathrm{s}$, and the controls are in inches.

Standard state perturbations ${ }^{1}$ are used to derive the li..._ar models presented here. Any deviation from these perturbations are clearly marked in the caption of the corresponding table.

\footnotetext{
'a defined in chapter 3
} 


\begin{tabular}{|c|c|c|c|c|c|c|c|c|}
\hline & \multicolumn{8}{|c|}{$1 \mathrm{knot}$} \\
\hline & $\theta_{R}$ & $\dot{\theta}_{R}$ & $\phi_{R}$ & $\dot{\phi}_{R}$ & $p$ & $q$ & $r$ & 4 \\
\hline$\dot{\theta}_{R}$ & 0.0000 & 1.0000 & 0.0000 & 0.0000 & 0.0000 & 0.0000 & 0.0000 & 0.0000 \\
\hline$\ddot{\theta}_{R}$ & 1.3778 & -28.1106 & -953.7717 & .67 .8515 & -69.9727 & -28.4630 & -3.1654 & 0.2654 \\
\hline$\dot{\phi}_{k}$ & 0.0000 & 0.0000 & 0.0000 & 1.0000 & 0.0000 & 0.0000 & 0.0000 & 0.0000 \\
\hline$\ddot{\phi}_{R}$ & 948.6362 & 67.8038 & .8 .2484 & -27.9589 & -28.1406 & 69.9424 & -4.0287 & -0.9506 \\
\hline$\dot{\boldsymbol{p}}$ & 7.4191 & 0.0447 & 12.1182 & -0.2191 & -0.4263 & 0.0720 & 0.6598 & -0.0055 \\
\hline$\dot{q}$ & 2.9253 & -0.0598 & -2.0295 & -0.0069 & -0.0160 & -0.2278 & 0.0062 & -0.0171 \\
\hline$\dot{r}$ & -2.6388 & 0.1156 & 5.5016 & 0.0777 & 0.5505 & 0.0935 & -2.1630 & 0.0051 \\
\hline$\dot{u}_{F}$ & -5.4903 & 0.1126 & 3.8205 & 0.0134 & 0.0297 & 0.1011 & -0.0528 & -0.0148 \\
\hline$\dot{v}_{F}$ & 3.8348 & 0.0130 & 5.5116 & -0.1132 & -0.2981 & 0.0298 & 0.3895 & -0.0042 \\
\hline$\dot{w}_{F}$ & -0.4737 & 0.0099 & 0.3337 & .0 .0065 & -0.1377 & 0.4559 & 0.8668 & 0.0253 \\
\hline$\dot{\phi}$ & 0.0000 & 0.0000 & 0.0000 & 0.0000 & 1.0000 & -0.0011 & 0.0596 & 0.0000 \\
\hline$\dot{\boldsymbol{\theta}}$ & 0.0000 & 0.0000 & 0.0000 & 0.0000 & 0.0000 & 0.9998 & 0.0189 & 0.0000 \\
\hline$\dot{v}_{i_{\text {TR }}}$ & .0 .0270 & 0.0000 & 0.0041 & 0.0000 & -93.8427 & -7.7374 & 399.3455 & 0.3751 \\
\hline$\dot{\lambda}_{0}$ & 0.0096 & .0 .0000 & 0.0000 & 0.0001 & 0.0034 & 0.0022 & -0.0147 & -0.0028 \\
\hline$\dot{\lambda}$, & .4 .8532 & 0.0000 & -0.0000 & 0.1431 & 0.0750 & -0.0116 & 0.0051 & 0.0048 \\
\hline$\dot{\lambda}_{c}$ & 0.0009 & 0.1431 & 4.8537 & -0.0000 & 0.0120 & 0.0751 & -0.0037 & 0.0316 \\
\hline \multicolumn{9}{|c|}{ Trim States: } \\
\hline & 1.7135 & 0.0000 & -1.6155 & 0.0000 & 0.0000 & 0.0000 & 0.0000 & 0.5135 \\
\hline
\end{tabular}

Table E.1: The RBM parameters at 1 knot (1/3) 


\begin{tabular}{|c|c|c|c|c|c|c|c|c|}
\hline & \multicolumn{8}{|c|}{$1 \mathrm{knot}$} \\
\hline & $v_{F}$ & $w_{r}$ & $\Phi$ & $\boldsymbol{\theta}$ & $v_{i_{T R}}$ & $\lambda_{0}$ & $\lambda$, & $\lambda_{c}$ \\
\hline$\dot{\theta}_{R}$ & 0.0000 & 0.0000 & 0.0000 & 0.0000 & 0.0000 & 0.0000 & 0.0000 & 0.0000 \\
\hline$\overline{\ddot{\theta}_{R}}$ & -0.9124 & 0.0298 & 0.0067 & -27.3593 & 0.0007 & -7.3826 & -0.3672 & 958.5659 \\
\hline$\dot{\phi}_{k}$ & 0.0000 & 0.0000 & 0.0000 & 0.0000 & 0.0000 & 0.0000 & 0.0000 & 0.0000 \\
\hline$\ddot{\phi}_{R}$ & -0.1781 & -0.0418 & -27.2123 & -0.0521 & 0.0561 & -10.2126 & 949.7490 & 7.2793 \\
\hline$\dot{\boldsymbol{p}}$ & -0.0931 & -0.0618 & -0.2136 & 0.0428 & -0.0764 & 15.0656 & 7.4346 & -9.3486 \\
\hline$\dot{\boldsymbol{q}}$ & -0.0477 & -0.0026 & -0.0067 & -0.0595 & -0.0007 & 6.0290 & 0.3669 & -0.9661 \\
\hline$\dot{\boldsymbol{r}}$ & 0.2372 & -0.0030 & 0.0719 & 0.1086 & 0.2290 & 1.7709 & -2.6375 & -5.0951 \\
\hline$\dot{u}_{\boldsymbol{r}}$ & 0.0052 & 0.0573 & 0.0130 & .9 .6797 & 1.7091 & -14.1323 & -0.7030 & 3.3744 \\
\hline$\dot{v}_{r}$ & -0.1312 & -0.0310 & 9.6773 & 0.0339 & -0.0971 & 7.1587 & 3.8424 & -4.1467 \\
\hline$\dot{w}_{F}$ & -0.0616 & -1.0508 & 0.1788 & -0.5789 & 0.0861 & 268.3364 & 0.1990 & -134.1748 \\
\hline$\dot{\Phi}$ & 0.0000 & 0.0000 & -0.0000 & 0.0000 & 0.0000 & 0.0000 & 0.0000 & 0.0000 \\
\hline$\dot{\boldsymbol{\theta}}$ & 0.0000 & 0.0000 & 0.0000 & 0.0000 & 0.0000 & 0.0000 & 0.0000 & 0.0000 \\
\hline$\dot{v}_{i \beta}$ & -45.6476 & -1.2708 & 0.0000 & 0.0146 & -60.5218 & -49.7707 & -0.0006 & 23.9353 \\
\hline$\dot{\lambda}_{0}$ & 0.0010 & 0.0358 & 0.0001 & 0.0027 & 0.0000 & .13 .5528 & -0.0045 & 6.6186 \\
\hline$\dot{\lambda}_{s}$ & -0.0317 & 0.0014 & 0.4579 & -0.0000 & 0.0000 & 0.0022 & -15.6843 & -0.1478 \\
\hline$\dot{\lambda}_{c}$ & 0.0049 & 0.0036 & 0.0000 & 0.4584 & 0.0000 & -0.0103 & -0.0000 & -15.8469 \\
\hline \multicolumn{9}{|c|}{ Trim States: } \\
\hline & -0.0006 & 0.3060 & -1.0833 & 3.4108 & 12.0360 & 0.0372 & -0.0001 & 0.0016 \\
\hline
\end{tabular}

Table E.2: The RBM parameters at 1 knot (2/3) 


\begin{tabular}{|c|c|c|c|c|}
\hline & \multicolumn{4}{|c|}{$1 \mathrm{knot}$} \\
\hline & $\theta_{\mathrm{MR}_{\mathrm{R}}}$ & A. & $A_{c}$ & $\theta_{q_{R}}$ \\
\hline$\dot{\theta}_{n}$ & 0.0000 & 0.0000 & 0.0000 & 0.0000 \\
\hline$\ddot{\theta}_{R}$ & 2.4853 & $\cdot 2.4523$ & .953 .4418 & 0.2759 \\
\hline$\phi_{k}$ & 0.0000 & 0.0000 & 0.0000 & 0.0000 \\
\hline$\ddot{\phi}_{R}$ & -0.1589 & -948.2837 & 8.8751 & $-8.2 \tau 25$ \\
\hline$\dot{\boldsymbol{p}}$ & -7.6328 & -7.4506 & -9.1150 & 11.1055 \\
\hline$\dot{q}$ & -0.8940 & 2.4540 & -2.0287 & -0.2759 \\
\hline$\dot{\boldsymbol{r}}$ & 13.2794 & 2.6341 & 2.3680 & -32.0203 \\
\hline$\dot{u}_{\text {r }}$ & 9.3144 & -4.5971 & 3.8205 & -0.0090 \\
\hline$\dot{v}_{F}$ & .4 .7553 & -3.8500 & -4.6445 & 13.3202 \\
\hline$\dot{w}_{\boldsymbol{F}}$ & -162.5201 & -0.9220 & 0.3348 & -0.0191 \\
\hline$\dot{\phi}$ & 0.0000 & 0.0000 & 0.0000 & 0.0000 \\
\hline$\dot{\boldsymbol{\theta}}$ & 0.0000 & 0.0000 & 0.0000 & 0.0000 \\
\hline$\dot{v}_{i_{i p R}}$ & 0.0000 & 0.0000 & 0.0000 & 4597.5533 \\
\hline$\dot{\lambda}_{0}$ & 2.7756 & 0.0088 & -0.0000 & 0.0000 \\
\hline$\dot{\lambda}_{1}$ & 0.0266 & 4.8454 & -0.0000 & 0.0000 \\
\hline$\dot{\lambda}_{c}$ & -0.0010 & -0.0000 & 4.8454 & 0.0000 \\
\hline \multicolumn{5}{|c|}{ Trim States: } \\
\hline & 10.5386 & 0.9063 & 1.1189 & 1.5313 \\
\hline
\end{tabular}

Table E.3: The RBM parameters at 1 knot (3/3) 


\begin{tabular}{|c|c|c|c|c|c|c|c|c|}
\hline & \multicolumn{8}{|c|}{$1 \mathrm{knot}$} \\
\hline & $\theta_{R}$ & $\dot{\theta}_{R}$ & $\phi_{R}$ & $\dot{\phi}_{n}$ & $p$ & $q$ & $r$ & $u_{F}$ \\
\hline$\dot{\theta}_{R}$ & 0.0000 & 1.0000 & 0.0000 & 0.0000 & 0.0000 & 0.0000 & 0.0000 & 0.0000 \\
\hline$\ddot{\theta}_{R}$ & 1.3779 & -28.1106 & -953.7715 & .67 .8515 & -69.9821 & -28.4691 & -3.0256 & 0.2650 \\
\hline$\dot{\phi}_{R}$ & 0.0000 & 0.0000 & 0.0000 & 1.0000 & 0.0000 & 0.0000 & 0.0000 & 0.0000 \\
\hline$\ddot{\alpha}$ & 948.6994 & 67.8040 & -8.2365 & -27.9607 & -28.1468 & 69.9417 & -3.9718 & -0.9498 \\
\hline$\dot{\boldsymbol{p}}$ & 7.3781 & 0.0445 & 12.1036 & -0.2179 & -0.4250 & 0.0719 & 0.6611 & -0.0055 \\
\hline$\dot{q}$ & 2.9223 & -0.0598 & -2.0294 & -0.0069 & -0.0162 & -0.2225 & 0.0054 & -0.0167 \\
\hline$\dot{r}$ & .2 .9002 & 0.1155 & 5.4975 & 0.0854 & 0.5583 & 0.0935 & -2.1605 & 0.0042 \\
\hline$u_{k}$ & -5.4836 & 0.1126 & 3.8205 & 0.0133 & 0.0293 & 0.1020 & .0 .0516 & -0.0174 \\
\hline$\dot{v}_{F}$ & 3.8348 & 0.0129 & 5.5047 & -0.1132 & -0.2993 & 0.0295 & 0.3884 & -0.0038 \\
\hline$\dot{w}_{F}$ & -0.4727 & 0.0099 & 0.3337 & -0.0065 & -0.1371 & 0.4643 & 0.8664 & 0.0230 \\
\hline$\dot{\phi}$ & 0.0000 & 0.0000 & 0.0000 & $0.00 c 0$ & 1.0000 & -0.0011 & 0.0572 & 0.0000 \\
\hline$\dot{\boldsymbol{\theta}}$ & 0.0000 & 0.0000 & 0.0000 & 0.0000 & 0.0000 & 0.9998 & 0.0189 & 0.0000 \\
\hline$\dot{v}_{i R}$ & -0.0267 & 0.0000 & 0.0036 & 0.0000 & -93.8267 & .7 .7167 & 399.2753 & 0.3731 \\
\hline$\dot{\lambda}_{0}$ & 0.0096 & -0.0000 & 0.0000 & 0.0001 & 0.0034 & 0.0020 & -0.0147 & -0.0028 \\
\hline$\dot{\lambda}_{\mathbf{s}}$ & -4.8532 & 0.0000 & -0.0000 & 0.1431 & 0.0751 & -0.0116 & 0.0048 & 0.0048 \\
\hline$\dot{\lambda}_{c}$ & $0.0 \mathrm{co9}$ & 0.1431 & 4.8537 & -0.0000 & 0.0120 & 0.0752 & -0.0037 & 0.0316 \\
\hline \multicolumn{9}{|c|}{ Trim States: } \\
\hline & 1.7135 & 0.0000 & -1.6155 & 0.0000 & 0.0000 & 0.0000 & 0.0000 & 0.5135 \\
\hline
\end{tabular}

Table E.4: The RBM parameters at 1 knot with $\Delta v_{\text {irR }}=0.01 \mathrm{~m} / \mathrm{s}(\mathrm{l} / 3)$ 


\begin{tabular}{|c|c|c|c|c|c|c|c|c|}
\hline & \multicolumn{8}{|c|}{$1 \mathrm{knot}$} \\
\hline & $v_{F}$ & $w_{F}$ & $\Phi$ & $\theta$ & $v_{\text {rR }}$ & $\lambda_{0}$ & $\lambda$, & $\lambda_{i}$ \\
\hline$\dot{\theta}_{R}$ & 0.0000 & 0.0000 & 0.0000 & 0.0000 & 0.0000 & 0.0000 & 0.0000 & 0.0000 \\
\hline$\ddot{\theta}_{R}$ & -0.9110 & 0.0270 & 0.0067 & .27 .3595 & 0.0007 & -6.5893 & -0.3656 & 958.1789 \\
\hline$\dot{\phi}_{R}$ & 0.0000 & 0.0000 & 0.0000 & 0.0000 & 0.0000 & 0.0000 & 0.0000 & 0.0000 \\
\hline$\ddot{\phi}_{R}$ & -0.1779 & -0.0417 & -27.2141 & -0.0520 & 0.0561 & -10.2211 & 949.8122 & 7.2780 \\
\hline$\dot{p}$ & -0.0932 & -0.0618 & -0.2124 & 0.0427 & -0.0764 & 15.0575 & 7.3937 & -9.3392 \\
\hline$\dot{q}$ & -0.0484 & 0.0005 & -0.0067 & -0.0593 & -0.0007 & 5.1613 & 0.3653 & -0.5422 \\
\hline$\dot{r}$ & 0.2370 & -0.0030 & 0.0794 & 0.1086 & 0.2290 & 1.7514 & -2.8989 & -5.0827 \\
\hline$\dot{u}_{F}$ & 0.0053 & 0.0547 & 0.0129 & -9.6810 & 8.5465 & -13.6837 & -0.7000 & $\$ .1494$ \\
\hline$\dot{v}_{F}$ & -0.1310 & -0.0309 & 9.6786 & 0.0330 & -0.1033 & 7.1592 & 3.8424 & -4.1445 \\
\hline$\dot{w}_{f}$ & -0.0621 & -1.0498 & 0.1786 & -0.5559 & 0.4137 & 267.9446 & 0.1993 & -133.9861 \\
\hline$\dot{\boldsymbol{\phi}}$ & 0.0000 & 0.0000 & -0.0000 & -0.0000 & 0.0000 & 0.0000 & 0.0000 & 0.0000 \\
\hline$\dot{\theta}$ & 0.0000 & 0.0000 & 0.0000 & 0.0000 & 0.0000 & 0.0000 & 0.0000 & 0.0000 \\
\hline$\dot{v}_{\dot{i}_{A}}$ & -45.6503 & -1.2705 & 0.0000 & 0.0146 & -60.5056 & -49.6817 & -0.0006 & 23.8915 \\
\hline$\dot{\lambda}_{0}$ & 0.0010 & 0.0358 & 0.0001 & 0.0027 & 0.0000 & -13.5487 & -0.0045 & 6.6166 \\
\hline$\dot{\lambda}$, & -0.0317 & 0.0014 & 0.4578 & -0.0000 & 0.0000 & 0.0027 & -15.6795 & -0.1480 \\
\hline$\dot{\lambda}_{c}$ & 0.0049 & 0.0036 & 0.0000 & 0.4583 & 0.0000 & .0 .0115 & -0.0000 & -15.841 .5 \\
\hline \multicolumn{9}{|c|}{ Trim States: } \\
\hline & -0.0006 & 0.3060 & -1.0833 & 3.4108 & 12.0360 & 0.0372 & -0.0001 & 0.0016 \\
\hline
\end{tabular}

Table E.5: The RBM parameters at 1 knot with $\Delta v_{i R}=0.01 \mathrm{~m} / \mathrm{s}(2 / 3)$ 


\begin{tabular}{|c|c|c|c|c|}
\hline & \multicolumn{4}{|c|}{1 knot } \\
\hline & $\theta_{g_{R B}}$ & A, & $A_{c}$ & $\theta_{g_{R}}$ \\
\hline$\dot{\theta}_{R}$ & 0.0000 & 0.0000 & 0.0000 & 0.0000 \\
\hline$\ddot{\theta}_{R}$ & 2.4051 & -2.4517 & $-953.44 ! 7$ & 0.2762 \\
\hline$\dot{\phi}_{R}$ & 0.0000 & 0.0000 & 0.0000 & 0.0000 \\
\hline$\ddot{\phi}_{n}$ & -0.1483 & -948.3475 & 8.8711 & -8.2725 \\
\hline$\dot{p}$ & -7.6306 & -7.4095 & -9.1110 & 11.1055 \\
\hline$\dot{q}$ & -0.7168 & 2.4535 & -2.0287 & -0.2762 \\
\hline$\dot{r}$ & 13.2744 & 2.8966 & 2.3678 & -32.0203 \\
\hline$\dot{u}_{r}$ & 8.9807 & -4.5962 & 3.8205 & -0.0090 \\
\hline$\dot{v}_{F}$ & -4.7539 & -3.8500 & -4.6425 & 13.3202 \\
\hline$\dot{w}_{F}$ & -162.5494 & -0.9219 & 0.3348 & -0.0190 \\
\hline$\dot{\phi}$ & 0.0000 & 0.0000 & 0.0000 & 0.0000 \\
\hline$\dot{\theta}$ & 0.0000 & 0.0000 & 0.0000 & 0.0000 \\
\hline$\dot{v}_{i r \Omega}$ & 0.0000 & 0.0000 & 0.0000 & 4597.5604 \\
\hline$\dot{\lambda}_{0}$ & 2.7756 & 0.0088 & -0.0000 & 0.0000 \\
\hline$\dot{\lambda}_{2}$ & 0.0265 & 4.8454 & -0.0000 & 0.0000 \\
\hline$\dot{\lambda}_{c}$ & -0.0010 & -0.0000 & 4.8454 & 0.0000 \\
\hline \multicolumn{5}{|c|}{ Trim States: } \\
\hline & 10.5386 & 0.9063 & 1.1189 & 1.5313 \\
\hline
\end{tabular}

Table E.6: The RBM parameters at 1 knot with $\Delta v_{i_{R}}=0.01 \mathrm{~m} / \mathrm{s}(3 / 3)$ 


\begin{tabular}{|c|c|c|c|c|c|c|c|c|}
\hline & \multicolumn{8}{|c|}{40 knots } \\
\hline & $\theta_{R}$ & $\dot{\theta}_{R}$ & $\phi_{k}$ & $\dot{\phi}_{n}$ & $\boldsymbol{p}$ & $q$ & $r$ & $u_{t}$ \\
\hline$\dot{\theta}_{R}$ & 0.0000 & 1.0000 & 0.0000 & 0.0000 & 0.0000 & 0.0000 & 0.0000 & 0.0000 \\
\hline$\ddot{\theta}_{R}$ & -0.6137 & -28.1094 & -957.2151 & -67.8133 & -69.4866 & -28.4165 & -3.9294 & 0.2315 \\
\hline$\dot{\phi}_{n}$ & 0.0000 & 0.0000 & 0.0000 & 1.0000 & 0.0000 & 0.0000 & 0.0000 & 0.0000 \\
\hline$\ddot{\phi}_{R}$ & 945.6856 & 67.6514 & -13.8873 & -27.9558 & -28.2774 & 69.3201 & -3.2283 & -0.7168 \\
\hline$\dot{p}$ & 6.9246 & 0.1955 & 16.9055 & -0.2204 & -0.3706 & 0.2141 & 0.6598 & -0.0047 \\
\hline$\dot{q}$ & 4.1755 & -0.0608 & -2.0055 & -0.0448 & -0.0289 & -0.2685 & 0.0069 & 0.0086 \\
\hline$\dot{r}$ & -2.5602 & 0.1407 & 6.2949 & 0.0616 & 0.5305 & 0.1292 & -2.1209 & 0.0039 \\
\hline$\dot{u}_{f}$ & -7.6525 & 0.1145 & 3.7712 & 0.1031 & 0.1258 & -1.0980 & -0.0694 & -0.0140 \\
\hline$\dot{v}_{F}$ & 3.5875 & 0.0849 & 7.7965 & -0.1130 & 0.8993 & 0.0994 & -19.6711 & $\cdot 0.0038$ \\
\hline$\dot{w}_{F}$ & -0.7268 & 0.0102 & 0.3279 & -0.2964 & -0.3669 & 20.3756 & 0.6745 & 0.0684 \\
\hline$\dot{\Phi}$ & 0.0000 & 0.0000 & 0.0000 & 0.0000 & 1.0000 & -0.0006 & 0.0592 & 0.0000 \\
\hline$\dot{\boldsymbol{\theta}}$ & 0.0000 & 0.0000 & 0.0000 & 0.0000 & 0.0000 & 0.9999 & 0.0104 & 0.0000 \\
\hline$\dot{v}_{i R R}$ & 7.0268 & 0.0000 & 0.0372 & 0.0000 & -63.8752 & 21.4130 & 284.0840 & -4.8053 \\
\hline$\dot{\lambda}_{0}$ & 0.1307 & -0.0000 & 0.0001 & 0.0052 & 0.0070 & 0.0157 & $\cdot 0.0116$ & .0 .0085 \\
\hline$\dot{\lambda}_{,}$ & -4.8352 & 0.0000 & 0.0015 & 0.1430 & 0.1297 & .0 .0091 & 0.0112 & 0.0037 \\
\hline$\dot{\lambda}_{c}$ & 0.3969 & 0.1431 & 4.8714 & -0.0000 & 0.0099 & 0.1383 & -0.0038 & 0.0013 \\
\hline \multicolumn{9}{|c|}{ Trim States: } \\
\hline & 1.0378 & 0.0000 & -0.3379 & 0.0000 & 0.0000 & 0.0000 & 0.0000 & 20.5418 \\
\hline
\end{tabular}

Table E.7: The RBM parameters at 40 knots (1/3) 


\begin{tabular}{|c|c|c|c|c|c|c|c|c|}
\hline & \multicolumn{8}{|c|}{40 knots } \\
\hline & $v_{F}$ & $w_{r}$ & $\Phi$ & $\boldsymbol{\theta}$ & $v_{i r R}$ & $\lambda_{0}$ & $\lambda$, & $\lambda_{c}$ \\
\hline$\dot{\theta}_{R}$ & 0.0000 & 0.0000 & 0.0000 & 0.0000 & 0.0000 & 0.0000 & 0.0000 & 0.0000 \\
\hline$\ddot{\theta}_{R}$ & -0.7924 & 0.0545 & 0.0438 & -27.3874 & 0.0010 & -1.1718 & -1.6625 & 956.4744 \\
\hline$\dot{\phi}_{R}$ & 0.0000 & 0.0000 & 0.0000 & 0.0000 & 0.0000 & 0.0000 & 0.0000 & 0.0000 \\
\hline$\ddot{\phi}_{R}$ & -0.2025 & -0.6808 & -27.2093 & -0.1991 & 0.0561 & 153.0478 & 949.6439 & -69.2231 \\
\hline$\dot{\boldsymbol{p}}$ & -0.0809 & -0.0557 & -0.2148 & 0.1880 & -0.0769 & 13.4213 & 7.4764 & -13.5926 \\
\hline$\dot{\boldsymbol{q}}$ & 0.0105 & -0.0293 & -0.0435 & .0 .0312 & -0.0010 & 0.1507 & 1.6515 & 0.9510 \\
\hline$\dot{\boldsymbol{r}}$ & 0.2438 & -0.0438 & 0.0562 & 0.1356 & 0.2361 & 10.9478 & -2.0929 & -10.6247 \\
\hline$\dot{u}_{r}$ & 0.0095 & 0.0816 & 0.1002 & -9.6441 & 1.7108 & -18.9489 & -3.7464 & 4.5025 \\
\hline$\dot{v}_{r}$ & .0 .1050 & -0.0247 & 9.6790 & 0.0976 & -0.0995 & 6.0153 & 3.8326 & -5.9753 \\
\hline$\dot{w}_{\boldsymbol{F}}$ & -0.0106 & -1.0315 & -0.1866 & -0.5902 & 0.0860 & 249.3049 & 10.0336 & -124.2577 \\
\hline$\dot{\phi}$ & 0.0000 & 0.0000 & -0.0000 & -0.0000 & 0.0000 & 0.0000 & 0.0000 & 0.0000 \\
\hline$\dot{\theta}$ & 0.0000 & 0.0000 & 0.0000 & 0.0000 & 0.0000 & 0.0000 & 0.0000 & 0.0000 \\
\hline$\dot{v}_{i_{f R}}$ & -32.6601 & 1.4445 & 0.0033 & 6.0455 & .58 .5602 & -233.7192 & -0.1123 & -95.2481 \\
\hline$\dot{\lambda}_{0}$ & 0.0004 & 0.0231 & 0.0052 & 0.1528 & 0.0000 & -10.0738 & -0.1799 & -0.2768 \\
\hline$\dot{\lambda}_{1}$ & -0.0060 & 0.0038 & 0.3631 & 0.0002 & 0.0000 & -0.8344 & -12.6696 & 0.4393 \\
\hline$\dot{\lambda}_{c}$ & 0.0041 & 0.0193 & 0.0002 & 0.7438 & 0.0000 & 20.5594 & -0.0072 & -36.2153 \\
\hline \multicolumn{9}{|c|}{ Trim States: } \\
\hline & -0.0126 & 1.2161 & -0.5945 & 3.388 & 4.4555 & 0.0159 & -0.0000 & 0.0192 \\
\hline
\end{tabular}

Table E.8: The RBM parameters at 40 knots (2/3) 


\begin{tabular}{|c|c|c|c|c|}
\hline & \multicolumn{4}{|c|}{40 knots } \\
\hline & $\theta_{q_{R}}$ & A, & $A_{c}$ & $\theta_{a_{T R}}$ \\
\hline$\dot{\theta}_{R}$ & 0.0000 & 0.0000 & 0.0000 & 0.0000 \\
\hline$\ddot{\theta}_{R}$ & 1.6503 & -0.9726 & -956.9356 & -0.1231 \\
\hline$\dot{\phi}_{R}$ & 0.0000 & 0.0000 & 0.0000 & 0.0000 \\
\hline$\ddot{\phi}_{R}$ & -211.6339 & -958.0036 & 3.5100 & -8.4167 \\
\hline$\dot{p}$ & -6.7184 & -8.0357 & -3.7751 & 11.2840 \\
\hline$\dot{q}$ & -0.4890 & 1.0256 & -2.0024 & 0.1231 \\
\hline$\dot{r}$ & 6.8949 & 1.6549 & 3.2389 & -32.4056 \\
\hline$\dot{u}_{p}$ & 10.9676 & -0.6652 & 3.7702 & -0.2115 \\
\hline$\dot{v}_{\boldsymbol{r}}$ & -3.9986 & -4.0841 & -2.0967 & 13.4918 \\
\hline$\dot{w}_{F}$ & -163.1945 & -20.7468 & 0.3425 & 0.0480 \\
\hline$\dot{\boldsymbol{\Phi}}$ & 0.0000 & 0.0000 & 0.0000 & 0.0000 \\
\hline$\dot{\theta}$ & 0.0000 & 0.0000 & 0.0000 & 0.0000 \\
\hline$\dot{v}_{i_{r}}$ & 0.0000 & 0.0000 & 0.0000 & 4656.7947 \\
\hline$\dot{\lambda}_{0}$ & 2.8039 & 0.3534 & -0.0002 & 0.0000 \\
\hline$\dot{\lambda}_{2}$ & 1.1026 & 4.8979 & -0.0000 & 0.0000 \\
\hline$\dot{\lambda}_{c}$ & -0.0016 & -0.0001 & 4.8629 & 0.0000 \\
\hline \multicolumn{5}{|c|}{ Trim States: } \\
\hline & 9.2313 & 0.0406 & 1.1361 & 0.6975 \\
\hline
\end{tabular}

Table E.9: The RBM parameters at 40 knots $(3 / 3)$ 


\begin{tabular}{|c|c|c|c|c|c|c|c|c|}
\hline & \multicolumn{8}{|c|}{40 knots } \\
\hline & $\boldsymbol{\theta}_{\boldsymbol{R}}$ & $\dot{\theta}_{R}$ & $\phi_{n}$ & $\dot{\phi}_{\mathbf{R}}$ & $p$ & $q$ & $r$ & $u_{F}$ \\
\hline$\dot{\theta}_{R}$ & 0.0000 & 1.0000 & 0.0000 & 0.0000 & 0.0000 & 0.0000 & 0.0000 & 0.0000 \\
\hline$\ddot{\theta}_{R}$ & -0.6137 & -28.1094 & -957.2151 & .67 .8133 & -69.4866 & -28.4165 & -3.9294 & 0.2315 \\
\hline$\dot{\phi}_{k}$ & 0.0000 & 0.0000 & 0.0000 & 1.0000 & 0.0000 & 0.0000 & 0.0000 & 0.0000 \\
\hline$\ddot{\phi}_{n}$ & 945.6856 & 67.6514 & -13.8873 & -27.9558 & -28.2774 & 69.3201 & -3.2283 & -0.7168 \\
\hline$\dot{p}$ & 6.9246 & 0.1955 & 16.9055 & -0.2204 & -0.3706 & 0.2141 & 0.6598 & -0.0047 \\
\hline$\dot{q}$ & 4.1755 & -0.0608 & -2.0055 & -0.0448 & -0.0289 & -0.2685 & 0.0069 & 0.0086 \\
\hline$\dot{r}$ & -2.5602 & 0.1407 & 6.2949 & 0.0616 & 0.5305 & 0.1292 & -2.1209 & 0.0039 \\
\hline$\dot{u}_{F}$ & -7.6525 & 0.1145 & 3.7712 & 0.1031 & 0.1258 & -1.0980 & -0.0694 & -0.0140 \\
\hline$\dot{v}_{F}$ & 3.5875 & 0.0849 & 7.7965 & -0.1130 & 0.8993 & 0.0994 & -19.6711 & -0.0038 \\
\hline$\dot{w}_{F}$ & -0.7268 & 0.0102 & 0.3279 & -0.2964 & -0.3669 & 20.3756 & 0.6745 & 0.0684 \\
\hline$\dot{\phi}$ & 0.0000 & 0.0000 & 0.0000 & 0.0000 & 1.0000 & -0.0006 & 0.0592 & 0.0000 \\
\hline$\dot{\boldsymbol{\theta}}$ & 0.0000 & 0.0000 & 0.0000 & 0.0000 & 0.0000 & 0.9999 & 0.0104 & 0.0000 \\
\hline$\dot{v}_{i_{R}}$ & 7.0268 & 0.0000 & 0.0372 & 0.0000 & -63.8752 & 21.4130 & 284.0840 & -4.8053 \\
\hline$\dot{\lambda}_{0}$ & 0.1307 & -0.0000 & 0.0001 & 0.0052 & 0.0070 & 0.0157 & -0.0116 & -0.0085 \\
\hline$\dot{\lambda}_{s}$ & -4.8352 & 0.0000 & 0.0015 & 0.1430 & 0.1297 & .0 .0091 & 0.0112 & 0.0037 \\
\hline$\dot{\lambda}_{c}$ & 0.3969 & 0.1431 & 4.8714 & -0.0000 & 0.0099 & 0.1383 & -0.0038 & 0.0013 \\
\hline \multicolumn{9}{|c|}{ Trim States: } \\
\hline & 1.0378 & 0.0000 & -0.3379 & 0.0000 & 0.0000 & 0.0000 & 0.0000 & 20.5418 \\
\hline
\end{tabular}

Table E.10: The RBM parameters at 40 knots with $\Delta \bar{\phi}=0.01$ deg $(1 / 3)$ 


\begin{tabular}{|c|c|c|c|c|c|c|c|c|}
\hline & \multicolumn{8}{|c|}{40 knots } \\
\hline & $v_{F}$ & $w_{F}$ & $\Phi$ & $\boldsymbol{\theta}$ & $v_{i R R}$ & $\lambda_{0}$ & $\lambda$, & $\lambda$. \\
\hline$\dot{\theta}_{R}$ & 0.0000 & 0.0000 & 0.0000 & 0.0000 & 0.0000 & 0.0000 & 0.0000 & 0.0000 \\
\hline$\ddot{\theta}_{R}$ & -0.7924 & 0.0545 & 4.3843 & -2738.7360 & 0.0010 & -1.1718 & -1.6625 & 956.47 .44 \\
\hline$\phi_{R}$ & 0.0000 & 0.0000 & 0.0000 & 0.0000 & 0.0000 & 0.0000 & 0.0000 & 0.0000 \\
\hline$\ddot{\phi}_{R}$ & -0.2025 & -0.6808 & -2720.9286 & -19.9139 & 0.0561 & 153.0478 & 949.6439 & -69.2231 \\
\hline$\dot{\boldsymbol{p}}$ & -0.0809 & -0.0557 & -21.4811 & 18.8036 & .0 .0769 & 13.4213 & 7.4764 & .13 .5926 \\
\hline$\dot{q}$ & 0.0105 & -0.0293 & -4.3546 & -3.1222 & -0.0010 & 0.1507 & 1.6515 & 0.9510 \\
\hline$\dot{r}$ & 0.2438 & -0.0438 & 5.6244 & 13.5606 & 0.2361 & 10.9478 & -2.0929 & -10.6247 \\
\hline$\dot{u}_{F}$ & 0.0095 & 0.0816 & 10.0224 & 4.7366 & 0.0188 & -18.9489 & -3.7464 & 4.5025 \\
\hline$\dot{v}_{F}$ & -0.1050 & -0.0247 & -1.1922 & 8.1525 & -0.0986 & 6.0153 & 3.8326 & -5.9753 \\
\hline$\dot{w}_{F}$ & -0.0106 & -1.0315 & -28.7105 & -1.6611 & 0.0008 & 249.3049 & 10.0336 & $-124.257 i$ \\
\hline$\dot{\phi}$ & 0.0000 & 0.0000 & -0.0000 & -0.0000 & 0.0000 & 0.0000 & 0.0000 & 0.0000 \\
\hline$\dot{\boldsymbol{\theta}}$ & 0.0000 & 0.0000 & 0.0000 & 0.0000 & 0.0000 & 0.0000 & 0.0000 & 0.0000 \\
\hline$\dot{v}_{\text {trR }}$ & -32.6601 & 1.4445 & 0.3326 & 604.5461 & -58.5602 & -233.7192 & .0 .1123 & .95 .2481 \\
\hline$\dot{\lambda}_{0}$ & 0.0004 & 0.0231 & 0.5157 & 15.2844 & 0.0000 & -10.0738 & -0.1799 & .0 .2768 \\
\hline$\dot{\lambda}_{s}$ & -0.0060 & 0.0038 & 36.3138 & 0.0161 & 0.0000 & -0.8344 & -12.6696 & 0.439 .3 \\
\hline$\dot{\lambda}_{c}$ & 0.0041 & 0.0193 & 0.0210 & 74.3829 & 0.0000 & 20.5594 & -0.0072 & -36.2153 \\
\hline \multicolumn{9}{|c|}{ Trim States: } \\
\hline & -0.0126 & 1.2161 & .0 .5945 & 3.388 & 4.4555 & 0.0159 & -0.0000 & 0.0192 \\
\hline
\end{tabular}

Table E.11: The RBM parameters at 40 knots with $\Delta \bar{\phi}=0.01 \mathrm{deg}(2 / 3)$ 


\begin{tabular}{|c|c|c|c|c|}
\hline & \multicolumn{4}{|c|}{40 knots } \\
\hline & $\theta_{q_{1 R}}$ & A. & $A_{c}$ & $\theta_{a_{R}}$ \\
\hline$\dot{\theta}_{R}$ & 0.0000 & 0.0000 & 0.0000 & 0.0000 \\
\hline$\ddot{\theta}_{R}$ & 1.6503 & -0.9726 & -956.9356 & -0.1231 \\
\hline$\dot{\phi}_{R}$ & 0.0000 & 0.0000 & 0.0000 & 0.0000 \\
\hline$\ddot{\phi}_{n}$ & -211.6339 & -958.0036 & 3.5100 & -8.4167 \\
\hline$\dot{p}$ & -6.7184 & -8.0357 & -3.7751 & 11.2840 \\
\hline$\dot{q}$ & -0.4890 & 1.0256 & -2.0024 & 0.1231 \\
\hline$\dot{\boldsymbol{r}}$ & 6.8949 & 1.6549 & 3.2389 & .32 .4056 \\
\hline$\dot{u}_{F}$ & 10.9676 & -0.6652 & 3.7702 & -0.2115 \\
\hline$\dot{v}_{p}$ & -3.9986 & -4.0841 & -2.0967 & 13.4918 \\
\hline$\dot{w}_{\boldsymbol{F}}$ & -163.1945 & -20.7468 & 0.3425 & 0.0480 \\
\hline$\dot{\phi}$ & 0.0000 & 0.0000 & 0.0000 & 0.0000 \\
\hline$\dot{\theta}$ & 0.0000 & 0.0000 & 0.0000 & 0.0000 \\
\hline$\dot{v}_{i_{-R}}$ & 0.0000 & 0.0000 & 0.0000 & 4656.7947 \\
\hline$\dot{\lambda}_{0}$ & 2.8039 & 0.3534 & -0.0002 & 0.0000 \\
\hline$\dot{\lambda}_{2}$ & 1.1026 & 4.8979 & .0 .0000 & 0.0000 \\
\hline$\dot{\lambda}_{c}$ & -0.0016 & -0.0001 & 4.8629 & 0.0000 \\
\hline \multicolumn{5}{|c|}{ Trim States: } \\
\hline & 9.2313 & 0.0406 & 1.1361 & 0.6975 \\
\hline
\end{tabular}

Table E.12: The RBM parameters at 40 knots with $\Delta \bar{\phi}=0.01 \operatorname{deg}(3 / 3)$ 


\begin{tabular}{|c|c|c|c|c|c|c|c|c|}
\hline & \multicolumn{8}{|c|}{80 knots } \\
\hline & $\theta_{R}$ & $\dot{\theta}_{R}$ & $\phi_{R}$ & $\dot{\phi}_{\mathrm{n}}$ & $p$ & $q$ & $r$ & 4 \\
\hline$\dot{\theta}_{R}$ & 0.0000 & 1.0000 & 0.0000 & 0.0000 & 0.0000 & 0.0000 & 0.0000 & 0.0000 \\
\hline$\ddot{\theta}_{R}$ & -1.0193 & -28.1086 & -967.5865 & -67.1977 & -69.2442 & -28.2238 & -6.1490 & 0.2178 \\
\hline$\dot{\phi}_{k}$ & 0.0000 & 0.0000 & 0.0000 & 1.0000 & 0.0000 & 0.0000 & 0.0000 & 0.0000 \\
\hline$\ddot{\phi}_{k}$ & 935.4164 & 67.6229 & -15.0610 & -27.9384 & -28.1524 & 69.0583 & -4.2933 & .0 .5807 \\
\hline$\dot{\boldsymbol{p}}$ & 6.6181 & 0.2251 & 17.7522 & -0.2306 & -0.4058 & 0.2424 & 0.6521 & -0.0024 \\
\hline$\dot{q}$ & 4.3807 & -0.0615 & -2.0143 & -0.0602 & -0.0525 & -0.4527 & 0.0154 & 0.0165 \\
\hline$\dot{r}$ & -0.3206 & 0.1291 & 5.8551 & -0.0253 & 0.4474 & 0.1234 & -2.2758 & 0.0012 \\
\hline$\dot{u}_{*}$ & -8.1642 & 0.1158 & 3.7637 & 0.1502 & 0.1754 & -2.7097 & -0.0912 & $\cdot 0.02 .40$ \\
\hline$\dot{v}_{F}$ & 3.2659 & 0.1003 & 8.2482 & -0.1122 & 2.5200 & 0.1113 & .40 .1280 & -0.0017 \\
\hline$\dot{w}_{F}$ & -0.7592 & 0.0105 & 0.3183 & -0.5980 & -0.6634 & 40.6978 & 0.6010 & 0.0975 \\
\hline$\dot{\Phi}$ & 0.0000 & 0.0000 & 0.0000 & 0.0000 & 1.0000 & -0.0006 & 0.0691 & 0.0000 \\
\hline$\dot{\theta}$ & 0.0000 & 0.0000 & 0.0000 & 0.0000 & 0.0000 & 1.0000 & 0.0083 & 0.0000 \\
\hline$\dot{v}_{i_{T R}}$ & -0.0701 & 0.0000 & -0.0004 & 0.0000 & -60.4359 & 5.9161 & 274.9886 & .2 .1851 \\
\hline$\dot{\lambda}_{0}$ & 0.1421 & -0.0000 & 0.0001 & 0.0104 & 0.0123 & 0.0103 & -0.0104 & .0 .0058 \\
\hline$\dot{\lambda}_{3}$ & -4.7824 & -0.0000 & 0.0047 & 0.1431 & 0.1372 & -0.0078 & 0.0178 & 0.0030 \\
\hline$\dot{\lambda}_{c}$ & 0.4717 & 0.1431 & 4.9242 & -0.0000 & 0.0096 & 0.1449 & -0.0031 & -0.0014 \\
\hline \multicolumn{9}{|c|}{ Trim States: } \\
\hline & 0.7155 & 0.0000 & -0.2289 & 0.0000 & 0.0000 & 0.0000 & 0.0000 & 41.057 .5 \\
\hline
\end{tabular}

Table E.13: The RBM parameters at 80 knots (1/3) 


\begin{tabular}{|c|c|c|c|c|c|c|c|c|}
\hline & \multicolumn{8}{|c|}{80 knots } \\
\hline & $v_{f}$ & $w_{r}$ & $\Phi$ & $\theta$ & $v_{i=8}$ & $\lambda_{0}$ & $\lambda$, & $\lambda_{c}$ \\
\hline$\dot{\theta}_{R}$ & 0.0000 & 0.0000 & 0.0000 & 0.0000 & 0.0000 & 0.0000 & 0.0000 & 0.0000 \\
\hline$\ddot{\theta}_{R}$ & -0.7717 & 0.0914 & 0.0590 & -27.4013 & 0.0013 & -4.7848 & -2.1924 & 958.7622 \\
\hline$\dot{\phi}_{n}$ & 0.0000 & 0.0000 & 0.0000 & 0.0000 & 0.0000 & 0.0000 & 0.0000 & 0.0000 \\
\hline$\ddot{\phi}_{R}$ & -0.1813 & -1.3412 & -27.1924 & -0.2277 & 0.0555 & 319.1390 & 949.0563 & -151.2739 \\
\hline$\dot{\boldsymbol{p}}$ & -0.1109 & -0.0599 & -0.2247 & 0.2179 & -0.0777 & 14.6819 & 7.8229 & -15.2667 \\
\hline$\dot{\boldsymbol{q}}$ & 0.0079 & -0.0711 & -0.0585 & -0.0173 & -0.0013 & 4.8276 & 2.1737 & -1.8681 \\
\hline$\dot{r}$ & 0.2562 & -0.0556 & -0.0282 & 0.1217 & 0.2510 & 13.8043 & 0.8526 & -11.5658 \\
\hline$\dot{u}_{\boldsymbol{F}}$ & 0.0097 & 0.0855 & 0.1460 & -9.7085 & 1.7104 & -24.7905 & -5.3421 & 9.8903 \\
\hline$\dot{v}_{F}$ & -0.1111 & -0.0270 & 9.6737 & 0.1151 & -0.1061 & 6.6294 & 3.8066 & -6.8459 \\
\hline$\dot{w}_{F}$ & -0.0108 & -1.0511 & -0.4997 & -0.6915 & 0.1031 & 248.2936 & 20.2676 & -123.6116 \\
\hline$\dot{\phi}$ & 0.0000 & 0.0000 & 0.0000 & 0.0000 & -0.0000 & 0.0000 & 0.0000 & 0.0000 \\
\hline$\dot{\theta}$ & 0.0000 & 0.0000 & -0.0000 & 0.0000 & 0.0000 & 0.0000 & 0.0000 & 0.0000 \\
\hline$\dot{v}_{\dot{i_{R}}}$ & -31.5197 & -0.0118 & -0.0001 & -0.0918 & -81.2885 & 0.8940 & 0.0013 & 2.0096 \\
\hline$\dot{\lambda}_{0}$ & 0.0004 & 0.0203 & 0.0103 & 0.3488 & 0.0000 & -9.5041 & -0.3595 & -7.2947 \\
\hline$\dot{\lambda}$ & -0.0023 & 0.0071 & 0.5245 & 0.0004 & 0.0000 & $\cdot 1.6732$ & -18.3352 & 0.8648 \\
\hline$\dot{\lambda}_{e}$ & 0.0039 & 0.0113 & 0.0004 & 1.3655 & 0.0000 & 55.1264 & -0.0129 & -74.9665 \\
\hline \multicolumn{9}{|c|}{ Trim States: } \\
\hline & -0.0237 & 2.8388 & 0.6329 & 3.9554 & 2.1259 & 0.0081 & 0.0000 & 0.0110 \\
\hline
\end{tabular}

Table E.14: The RBM parameters at 80 knots (2/3) 


\begin{tabular}{|c|c|c|c|c|}
\hline & \multicolumn{4}{|c|}{80 knots } \\
\hline & $\theta_{\text {ons }}$ & A. & $A_{c}$ & $\theta_{9 R}$ \\
\hline$\dot{\theta}_{R}$ & 0.0000 & 0.0000 & 0.0000 & 0.0000 \\
\hline$\ddot{\theta}_{R}$ & 2.9583 & .0 .0207 & .967 .3349 & -0.1507 \\
\hline$\dot{i}_{k}$ & 0.0000 & 0.0000 & 0.0000 & 0.0000 \\
\hline$\ddot{\phi}_{R}$ & -428.0545 & -987.8830 & 2.6643 & -8.7018 \\
\hline$\dot{\boldsymbol{p}}$ & -7.8227 & -9.0554 & -2.9396 & 11.6685 \\
\hline$\dot{q}$ & -3.1508 & 0.0354 & -1.9980 & 0.1507 \\
\hline$\dot{r}$ & 4.3754 & -1.9743 & 2.7814 & -33.5296 \\
\hline$\dot{u}_{F}$ & 16.1084 & 2.4245 & 3.7613 & -0.3630 \\
\hline$\dot{v}_{F}$ & -4.5054 & -4.3656 & -1.6525 & 13.9658 \\
\hline$\dot{w}_{r}$ & -167.6928 & -41.1896 & 0.3529 & -0.0017 \\
\hline$\dot{\boldsymbol{\Phi}}$ & 0.0000 & 0.0000 & 0.0000 & 0.0000 \\
\hline$\dot{\boldsymbol{\theta}}$ & 0.0000 & 0.0000 & 0.0000 & 0.0000 \\
\hline$\dot{v}_{i_{r \Omega}}$ & 0.0000 & 0.0000 & 0.0000 & 4820.3934 \\
\hline$\dot{\lambda}_{0}$ & 2.8876 & 0.7070 & -0.0004 & 0.0000 \\
\hline$\dot{\lambda}_{s}$ & 2.2074 & 5.0562 & -0.0001 & 0.0000 \\
\hline$\dot{\lambda}_{c}$ & -0.0021 & -0.0003 & 4.9156 & 0.0000 \\
\hline \multicolumn{5}{|c|}{ Trim States: } \\
\hline & 9.1514 & $\cdot 1.3941$ & 0.9486 & 0.4530 \\
\hline
\end{tabular}

Table E.15: The RBM parameters at 80 knots (3/3) 


\begin{tabular}{|c|c|c|c|c|c|c|c|c|}
\hline & \multicolumn{8}{|c|}{80 knots } \\
\hline & $\theta_{R}$ & $\dot{\theta}_{R}$ & $\phi_{n}$ & $\dot{\phi}_{\mathbf{n}}$ & $p$ & $q$ & $r$ & $u_{r}$ \\
\hline$\dot{\theta}_{R}$ & 0.0000 & 1.0000 & 0.0000 & 0.0000 & 0.0000 & 0.0000 & 0.0000 & 0.0000 \\
\hline$\ddot{\theta}_{R}$ & -1.0193 & -28.1086 & -967.5865 & .67 .7977 & -69.2442 & -28.2238 & -6.1490 & 0.2178 \\
\hline$\dot{\phi}_{n}$ & 0.0000 & 0.0000 & 0.0000 & 1.0000 & 0.0000 & 0.0000 & 0.0000 & 0.0000 \\
\hline$\ddot{\phi}_{R}$ & 935.4164 & 67.6229 & -15.0610 & .27 .9384 & .28 .1524 & 69.0583 & -4.2933 & -0.5807 \\
\hline$\dot{\boldsymbol{p}}$ & 6.6181 & 0.2251 & 17.7522 & -0.2306 & -0.4058 & 0.2424 & 0.6521 & -0.0024 \\
\hline$\dot{q}$ & 4.3807 & -0.0615 & -2.0143 & -0.0602 & -0.0525 & -0.4527 & 0.0154 & 0.0165 \\
\hline$\dot{r}$ & -0.3206 & 0.1291 & 5.8551 & -0.0253 & 0.4474 & 0.1234 & -2.2758 & 0.0012 \\
\hline$\dot{u}_{r}$ & -8.1642 & 0.1158 & 3.7637 & 0.1502 & 0.1754 & -2.7097 & -0.0912 & -0.0240 \\
\hline$\dot{v}_{f}$ & 3.2659 & 0.1003 & 8.2482 & -0.1122 & 2.5200 & 0.1113 & -40.1280 & -0.0017 \\
\hline$\dot{w}_{F}$ & -0.7592 & 0.0105 & 0.3183 & .0 .5980 & -0.6634 & 40.6978 & 0.6010 & 0.0975 \\
\hline$\dot{\phi}$ & 0.0000 & 0.0000 & 0.0000 & 0.0000 & 1.0000 & -0.0006 & 0.0691 & 0.0000 \\
\hline$\dot{\boldsymbol{\theta}}$ & 0.0000 & 0.0000 & 0.0000 & 0.0000 & 0.0000 & 1.0000 & 0.0083 & 0.0000 \\
\hline$\dot{v}_{\dot{i} \Omega}$ & -0.0701 & $0.000 c$ & -0.0004 & 0.0000 & -60.4359 & 5.9161 & 274.9886 & -2.1851 \\
\hline$\dot{\lambda}_{0}$ & 0.1421 & .0 .0000 & 0.0001 & 0.0104 & 0.0123 & 0.0103 & -0.0104 & -0.0058 \\
\hline$\dot{\lambda}$ & .4 .7824 & .0 .0000 & 0.0047 & 0.1431 & 0.1372 & -0.0078 & 0.0178 & 0.0030 \\
\hline$\dot{\lambda}_{c}$ & 0.4717 & 0.1431 & 4.9242 & .0 .0000 & 0.0096 & 0.1449 & -0.0031 & -0.0014 \\
\hline \multicolumn{9}{|c|}{ Trim States: } \\
\hline & 0.7155 & 0.0000 & -0.2289 & 0.0000 & 0.0000 & 0.0000 & 0.0000 & 41.0575 \\
\hline
\end{tabular}

Table E.16: The RBM parameters at 80 knots with $\Delta \bar{\phi}=0.01 \mathrm{deg}(1 / 3)$ 


\begin{tabular}{|c|c|c|c|c|c|c|c|c|}
\hline & \multicolumn{8}{|c|}{80 knots } \\
\hline & $v_{F}$ & $w_{F}$ & $\Phi$ & $\theta$ & $v_{i s}$ & $\lambda_{0}$ & $\lambda$, & $\lambda_{i}$ \\
\hline$\dot{\theta}_{R}$ & 0.0000 & 0.0000 & 0.0000 & 0.0000 & 0.0000 & 0.0000 & 0.0000 & 0.0000 \\
\hline$\ddot{\theta}_{R}$ & -0.7717 & 0.0914 & 11.7993 & .5480 .2226 & 0.0013 & -4.7842 & -2.3244 & 958.82339 \\
\hline$\dot{\phi}_{R}$ & 0.0000 & 0.0000 & 0.0000 & 0.0000 & 0.0000 & 0.0000 & 0.0000 & 0.0000 \\
\hline$\ddot{\phi}_{R}$ & -0.1813 & -1.3412 & -5438.3125 & -45.3676 & 0.0555 & 319.1391 & 949.0670 & -150.9565 \\
\hline$\dot{p}$ & -0.1109 & .0 .0599 & -45.0641 & 43.4762 & -0.0777 & 14.6819 & 7.8220 & -15.5668 \\
\hline$\dot{q}$ & 0.0079 & -0.0711 & -11.7029 & -3.4962 & -0.0013 & 4.8270 & 2.3053 & -1.9230 \\
\hline$\dot{\boldsymbol{r}}$ & 0.2562 & -0.0556 & -6.3754 & 23.5997 & 0.2510 & 13.8043 & 0.8470 & -11.8537 \\
\hline$\dot{u}_{F}$ & 0.0097 & 0.0855 & 29.1924 & 5.0152 & 0.0192 & -24.7906 & .5 .5901 & 10.0133 \\
\hline$\dot{v}_{\boldsymbol{F}}$ & -0.1111 & -0.0270 & -12.0311 & 19.5323 & -0.1052 & 6.6294 & 3.8066 & $\cdot 6.9709$ \\
\hline$\dot{w}_{F}$ & -0.0108 & -1.0511 & -116.1876 & -3.7429 & 0.0012 & 248.2939 & 20.2462 & -123.60 .54 \\
\hline$\dot{\boldsymbol{\Phi}}$ & 0.0000 & 0.0000 & 0.0000 & 0.0000 & -0.0000 & 0.0000 & 0.0000 & 0.0000 \\
\hline$\dot{\boldsymbol{\theta}}$ & 0.0000 & 0.0000 & -0.0000 & 0.0000 & 0.0000 & 0.0000 & 0.0000 & 0.0000 \\
\hline$\dot{v}_{i_{T R}}$ & -31.5197 & -0.0118 & -0.0173 & -24.9110 & -81.2885 & 0.8729 & 0.0017 & 2.4125 \\
\hline$\dot{\lambda}_{0}$ & 0.0004 & 0.0203 & 2.0614 & 70.3410 & 0.0000 & -9.5070 & -0.3594 & -7.2713 \\
\hline$\dot{\lambda}_{3}$ & -0.0023 & 0.0071 & 104.5892 & 0.0771 & 0.0000 & -1.6732 & -18.3134 & 0.9058 \\
\hline$\dot{\lambda}_{c}$ & 0.0039 & 0.0113 & 0.0773 & 274.1796 & 0.0000 & 55.1203 & -0.0128 & -74.9049 \\
\hline \multicolumn{9}{|c|}{ Trim States: } \\
\hline & -0.0237 & 2.8388 & 0.6329 & 3.9554 & 2.1259 & 0.0081 & 0.0000 & 0.0110 \\
\hline
\end{tabular}

Table E.17: The RBM parameters at 80 knots with $\Delta \bar{\phi}=0.01 \mathrm{deg}(2 / 3)$ 


\begin{tabular}{|c|c|c|c|c|}
\hline & \multicolumn{4}{|c|}{1 knot } \\
\hline & $\theta_{g_{R R}}$ & A, & $A_{c}$ & $\theta_{2 R}$ \\
\hline$\dot{\theta}_{R}$ & 0.0000 & 0.0000 & 0.0000 & 0.0000 \\
\hline$\ddot{\theta}_{R}$ & 2.9583 & -0.0207 & -967.3349 & -0.1507 \\
\hline$\dot{\phi}_{k}$ & 0.0000 & 0.0000 & 0.0000 & 0.0000 \\
\hline$\ddot{\phi}_{R}$ & -428.0545 & -987.8830 & 2.6643 & -8.7018 \\
\hline$\dot{p}$ & -7.8227 & -9.0554 & 2.9396 & 11.6685 \\
\hline$\dot{q}$ & -3.1508 & 0.0354 & -1.9980 & 0.1507 \\
\hline$\dot{r}$ & 4.3754 & -1.9743 & 2.7814 & -33.5296 \\
\hline$\dot{u}_{k}$ & 16.1084 & 2.4245 & 3.7613 & -0.3630 \\
\hline$\dot{v}_{\boldsymbol{F}}$ & -4.5054 & -4.3656 & -1.6525 & 13.9658 \\
\hline$\dot{w}_{F}$ & -167.6928 & -41.1896 & 0.3529 & -0.0017 \\
\hline$\dot{\phi}$ & 0.0000 & 0.0000 & 0.0000 & 0.0000 \\
\hline$\dot{\boldsymbol{\theta}}$ & 0.0000 & 0.0000 & 0.0000 & 0.0000 \\
\hline$\dot{v}_{i R}$ & 0.0000 & 0.0000 & 0.0000 & 4820.3934 \\
\hline$\dot{\lambda}_{0}$ & 2.8876 & 0.7070 & -0.0004 & 0.0000 \\
\hline$\dot{\lambda}_{1}$ & 2.2074 & 5.0562 & .0 .0001 & 0.0000 \\
\hline$\dot{\lambda}_{c}$ & -0.0021 & -0.0003 & 4.9156 & 0.0000 \\
\hline \multicolumn{5}{|c|}{ Trim States: } \\
\hline & 1.53 & 1.43 & 0.70 & 0.60 \\
\hline
\end{tabular}

Table E.18: The RBM parameters at 80 knots with $\Delta \bar{\phi}=0.01 \mathrm{deg}(3 / 3)$ 
Appendix F

Linear Model Parameters (Part II) 
The following tables define the A and B state space matrices of the linsar RBM model with the longitudinal transmission mode $\left(x_{1}, x_{2}, x_{3}\right)$. The units of the state vector and control vector are as stated in Appendix E. 


\begin{tabular}{|c|c|c|c|c|c|c|c|c|}
\hline & \multicolumn{8}{|c|}{$1 \mathrm{kno}^{+}$} \\
\hline & $\theta_{R}$ & $\dot{\theta}_{R}$ & $\phi_{n}$ & $\dot{\phi}_{2}$ & $p$ & $q$ & $r$ & $u_{5}$ \\
\hline$\dot{\theta}_{R}$ & 0.0000 & 1.0000 & 0.0000 & 0.0000 & 0.0000 & 0.0000 & 0.0000 & 0.0000 \\
\hline$\ddot{\theta}_{R}$ & 4.2897 & -28.1704 & -955.8002 & -67.8582 & -69.9922 & -28.5384 & -3.0391 & 0.2685 \\
\hline$\dot{\phi}_{n}$ & 0.0000 & 0.0000 & 0.0000 & 1.0000 & 0.0000 & 0.0000 & 0.0000 & 0.0000 \\
\hline$\ddot{\phi}_{R}$ & 948.6994 & 67.8040 & -8.2365 & -27.9607 & -28.1468 & 69.9417 & -3.9718 & -0.9498 \\
\hline$\dot{p}$ & 7.3781 & 0.0445 & 12.1036 & -0.2179 & -0.4250 & 0.0719 & 0.6611 & -0.0055 \\
\hline$\dot{q}$ & 0.0105 & 0.0000 & -0.0007 & -0.0002 & -0.0062 & -0.1532 & 0.0188 & -0.0202 \\
\hline$\dot{r}$ & -2.9002 & 0.1155 & 5.4975 & 0.0854 & 0.5583 & 0.0935 & -2.1605 & 0.0042 \\
\hline$\dot{u}_{r}$ & 0.0002 & -0.0000 & .0 .0000 & 0.0007 & 0.0104 & -0.0285 & -0.0769 & -0.0109 \\
\hline$\dot{v}_{F}$ & 3.8348 & 0.0129 & 5.5047 & -0.1132 & -0.2993 & 0.0295 & 0.3884 & -0.0038 \\
\hline$\dot{w}_{F}$ & 0.0071 & 0.0000 & -0.0005 & -0.0076 & -0.1388 & 0.4529 & 0.8642 & 0.0235 \\
\hline$\dot{\phi}$ & 0.0000 & 0.0000 & 0.0000 & 0.0000 & 1.0000 & -0.0011 & 0.0572 & 0.0000 \\
\hline$\dot{\theta}$ & 0.0000 & 0.0000 & 0.0000 & 0.0000 & 0.0000 & 0.9998 & 0.0189 & 0.0000 \\
\hline$\dot{v}_{i R}$ & -0.0267 & 0.0000 & 0.0036 & 0.0000 & -93.8267 & -7.7167 & 399.2753 & 0.3731 \\
\hline$\dot{\lambda}_{0}$ & 0.0096 & -0.0000 & 0.0000 & 0.0001 & 0.0034 & 0.0020 & -0.0147 & -0.0028 \\
\hline$\dot{\lambda}_{s}$ & -4.8532 & 0.0000 & -0.0000 & 0.1431 & 0.0751 & -0.0116 & 0.0048 & 0.0048 \\
\hline$\dot{\lambda}_{c}$ & 0.0009 & 0.1431 & 4.8537 & .0 .0000 & 0.0120 & 0.0752 & -0.0037 & 0.0316 \\
\hline$x_{1}$ & 19976.6040 & -410.2209 & -13917.6500 & -46.0266 & -68.9574 & -475.3430 & -92.1999 & 23.7401 \\
\hline$x_{2}$ & 0.0000 & 0.0000 & 0.0000 & 0.0000 & 0.0000 & 0.0000 & 0.0000 & 0.0000 \\
\hline$x_{3}$ & 0.0000 & 0.0000 & 0.0000 & 0.0000 & 0.0000 & 0.0000 & 0.0000 & 0.0000 \\
\hline \multicolumn{9}{|c|}{ Trim States: } \\
\hline & 1.8314 & 0.0000 & -1.6149 & 0.0000 & 0.0000 & 0.0000 & 0.0000 & 0.5136 \\
\hline
\end{tabular}

Table F.1: The RBM parameters at 1 knot with transmission mode (1/3) 
1 knot

\begin{tabular}{|c|c|c|c|c|c|c|c|c|}
\hline & \multicolumn{8}{|c|}{1 knot } \\
\hline & $v_{F}$ & $w_{F}$ & $\Phi$ & $\theta$ & $v_{i R}$ & $\lambda_{0}$ & $\lambda$, & $\lambda_{c}$ \\
\hline$\dot{\theta}_{R}$ & 0.0000 & 0.0000 & 0.0000 & 0.0000 & 0.0000 & 0.0000 & 0.0000 & 0.0000 \\
\hline$\ddot{\theta}_{R}$ & .0 .9129 & 0.0443 & 0.0002 & -27.4176 & 0.0007 & -10.8101 & -0.0059 & 962.2521 \\
\hline$\dot{\phi}_{n}$ & 0.0000 & 0.0000 & 0.0000 & 0.0000 & 0.0000 & 0.0000 & 0.0000 & 0.0000 \\
\hline$\ddot{\phi}_{R}$ & -0.1779 & -0.0417 & -27.2141 & -0.0520 & 0.0561 & -10.2211 & 949.8122 & 7.2780 \\
\hline$\dot{\boldsymbol{p}}$ & -0.0932 & -0.0618 & -0.2124 & 0.0427 & -0.0764 & 15.0575 & 7.3937 & -9.3392 \\
\hline$\dot{q}$ & -0.0465 & -0.0168 & -0.0002 & -0.0012 & -0.0007 & 9.3820 & 0.0056 & -4.6154 \\
\hline$\dot{\boldsymbol{r}}$ & 0.2370 & -0.0030 & 0.0794 & 0.1086 & 0.2290 & 1.7514 & -2.8989 & -5.0827 \\
\hline$\dot{u}_{f}$ & 0.0017 & 0.0874 & 0.0006 & -9.7905 & 0.0229 & -21.6325 & -0.0226 & 10.8201 \\
\hline$\dot{v}_{F}$ & -0.1310 & -0.0309 & 9.6786 & 0.0330 & -0.0955 & 7.1592 & 3.8424 & -4.1445 \\
\hline$\dot{w}_{\boldsymbol{F}}$ & -0.0624 & -1.0469 & 0.1776 & -0.5655 & 0.0003 & 267.2492 & 0.2585 & -133.3150 \\
\hline$\dot{\phi}$ & 0.0000 & 0.0000 & -0.0000 & -0.0000 & 0.0000 & 0.0000 & 0.0000 & 0.0000 \\
\hline$\dot{\boldsymbol{\theta}}$ & 0.0000 & 0.0000 & 0.0000 & 0.0000 & 0.0000 & 0.0000 & 0.0000 & 0.0000 \\
\hline$\dot{v}_{\text {rR }}$ & -45.6503 & -1.2705 & 0.0000 & 0.0146 & -60.5067 & -49.6817 & -0.0006 & 23.8915 \\
\hline$\dot{\lambda}_{0}$ & 0.0010 & 0.0358 & 0.0001 & 0.0027 & 0.0000 & -13.5487 & -0.0045 & 6.6166 \\
\hline$\dot{\lambda}$ & -0.0317 & 0.0014 & 0.4578 & -0.0000 & 0.0000 & 0.0027 & -15.6795 & -0.1480 \\
\hline$\dot{\lambda}_{c}$ & 0.0049 & 0.0036 & 0.0000 & 0.4583 & 0.0000 & -0.0115 & -0.0000 & -15.8415 \\
\hline$x_{1}$ & -12.9854 & 119.1873 & -44.7463 & -398.8099 & 0.0000 & -28956.0320 & 2467.7828 & 27943.4510 \\
\hline$x_{2}$ & 0.0000 & 0.0000 & 0.0000 & 0.0000 & 0.0000 & 0.0000 & 0.0000 & 0.0000 \\
\hline$x_{3}$ & 0.0000 & 0.0000 & 0.0000 & 0.0000 & 0.0000 & 0.0000 & 0.0000 & 0.0000 \\
\hline \multicolumn{9}{|c|}{ Trim States: } \\
\hline & -0.0006 & 0.0294 & $\cdot 1.0825$ & 3.2771 & 1.2029 & 0.0371 & -0.0001 & 0.0016 \\
\hline
\end{tabular}

Table F.2: The RBM parameters at 1 knot with transmission mode (2/3) 


\begin{tabular}{|c|c|c|c|c|c|c|c|}
\hline & \multicolumn{7}{|c|}{$1 \mathrm{knot}$} \\
\hline & $x_{1}$ & $x_{2}$ & $x_{3}$ & $\theta_{\text {YRR }}$ & A, & $A_{c}$ & $\theta_{g_{R}}$ \\
\hline$\dot{\theta}_{R}$ & 0.0000 & 0.0000 & 0.0000 & 0.0000 & 0.0000 & 0.0000 & 0.0000 \\
\hline$\ddot{\theta}_{R}$ & -0.0048 & -0.0424 & -1.0204 & 5.1667 & 0.0128 & -955.4705 & 0.2763 \\
\hline$\dot{\phi}_{n}$ & 0.0000 & 0.0000 & 0.0000 & 0.0000 & 0.0000 & 0.0000 & 0.0000 \\
\hline$\ddot{\phi}_{n}$ & 0.0000 & 0.0000 & -0.0105 & -0.1483 & -948.3475 & 8.8711 & -8.2725 \\
\hline$\dot{p}$ & 0.0000 & 0.0000 & 0.0143 & -7.6306 & $-\mathrm{T} .4095$ & -9.1110 & 11.1055 \\
\hline$\dot{q}$ & 0.0048 & 0.0424 & 1.0204 & -3.4783 & -0.0111 & 0.0000 & -0.2763 \\
\hline$\dot{r}$ & 0.0000 & 0.0000 & -0.0426 & 13.2744 & 2.8966 & 2.3678 & -32.0204 \\
\hline$\dot{u}_{f}$ & -0.0090 & -0.0799 & -1.9214 & 14.1814 & 0.0451 & -0.0000 & -0.0090 \\
\hline$\dot{v}_{F}$ & 0.0000 & 0.0000 & 0.0178 & -4.7539 & -3.8500 & -4.6425 & 13.3202 \\
\hline$\dot{w}_{F}$ & -0.0008 & -0.0070 & -0.1680 & -162.0943 & .0 .5158 & 0.0006 & -0.0189 \\
\hline$\dot{\boldsymbol{\Phi}}$ & 0.0000 & 0.0000 & 0.0000 & 0.0000 & 0.0000 & 0.0000 & 0.0000 \\
\hline$\dot{\boldsymbol{\theta}}$ & 0.0000 & 0.0000 & 0.0000 & 0.0000 & 0.0000 & 0.0000 & 0.0000 \\
\hline$\dot{v}_{i R}$ & 0.0000 & 0.0000 & 10.4938 & 0.0000 & 0.0000 & 0.0000 & 4597.5618 \\
\hline$\dot{\lambda}_{0}$ & 0.0000 & 0.0000 & 0.0000 & 2.7756 & 0.0088 & -0.0000 & 0.0000 \\
\hline$\dot{\lambda}$, & 0.0000 & 0.0000 & 0.0000 & 0.0265 & 4.8454 & -0.0000 & 0.0000 \\
\hline$\dot{\lambda}_{c}$ & 0.0000 & 0.0000 & 0.0000 & -0.0010 & -0.0000 & 4.8454 & 0.0000 \\
\hline$x_{1}$ & -38.4549 & -299.0346 & -6999.2717 & 18945.3960 & 16907.7740 & -13917.6700 & 0.0000 \\
\hline$x_{2}$ & 1.0000 & 0.0000 & 0.0000 & 0.0000 & 0.0000 & 0.0000 & 0.0000 \\
\hline$x_{3}$ & 0.0000 & 1.0000 & 0.0000 & 0.0000 & 0.0000 & 0.0000 & 0.0000 \\
\hline \multicolumn{8}{|c|}{ Trim States: } \\
\hline & 0.0000 & 0.0000 & 0.1681 & 10.5354 & 0.97016 & 1.1190 & 1.5301 \\
\hline
\end{tabular}

Table F.3: The RBM parameters at 1 knot with transmission mode (3/3) 


\section{References}

[1] Hall, W. E. Jr.; Bryson, A. E. Jr., "Inclusion of Rotor Dynamics in Controller Design of Helicopters", Journal of Aircraft Vol. 10, No. 4, April 1973.

[2] Takahashi, M. D., “A Flight-Dynamic Helicopter Mathematical Model with a Single Flap-Lag-Torsion Main Rotor", NASA TM 102267, Feb. 1990.

[3] Heffley, Robert K.; Jewell, Wayne F.; Lehman, John M.; Van Winkle, Richard A., "A Compilation and Analysis of Helicopter Handling Qualities Data, Volume One: Data Compilation”, NASA C.R. 3144, August 1979.

[4] Sattler, D.E., "The National Aeronautical Establishment Airborne Simulation Facility", NRC NAE MISC 58, May 1984.

[5] Yen, Jin; McLarty, Tyce T., "Analysis of Rotor-Fuselage Coupling and its Effects on Rotorcraft Stability and Responsen, Vertica Vol. 3, pp. 205 to 219, 1979.

[6] Gould, D.G.;Hindson, W.S., "Estimation of the Lateral-Directional Stability Derivatives of a Helicopter from Flight Measurements", NRC LR-S72, NRC No. 13882, Ottawa, Dec. 1973.

[7] deLeeuw, J.H.;Hui, K., "The Application of Linear Maximum Likelihood Estimation of Aerodynamic Derivatives for the Bell-205 and Bell-206", NAE AN-48, NRC No. 28442, Ottawa, October 1987.

[8] Hui, K.;Baillie, S., "Improving Prediction: The Incorporation of Simplified Rotor Dynamics in a Mathematical Model of the Bell 412HP", Canadian Aeronautics and Space Journal, Vol 40, No. 4, Dec. 1994. 
[9] Chen. Robert T. N., "Effects of Primary Rotor Parameters on Flapping Dynamics". NASA TP $1431,1980$.

[10] Ellis, Charles W., "Effects of Articulated Rotor Dynamics on Helicopter Automatic Control System Requirements", Aeronautical Engineering Review Vol. 12, No. T. July 1953.

[11] Heimbold, R. L.; Griffith, C. D., "Synthesis of an Electromechanical Control System for a Compound Hingeless Rotor Helicopter”, JAHS ${ }^{1}$ 17-2, April 1972.

[12] Hall, Wilton E. Jr., "Computational Methods for the Synthesis of Rotary-Wing VTOL Aircraft Control Systems", Ph.D. Dissertation August 1971, Stanford Univ., Stanford, Calif.

[13] Hansen, Raymond S., "Toward a Better Understanding of Helicopter Stability Deriva. tives", JAHS Vol. 29, No. 1, 1984.

[14] Peters, David A.; HaQuang, Ninh, "Dynamic Inflow for Practical Applications", JAHS Technical Note, October 1988.

[15] Ormiston, Robert A., “Application of Simplified Inflow Models to Rotorcraft Dynamic Analysis", JAHS Vol. 21, No. 3, Juiy 1976. (Technical Notes)

[16] Gaonkar, G. H.; Peters, David A., "Review of Dynamic Inflow Modeling for Rotorcraft Flight Dynamics", AIAA No. 86-0845, 1986.

[17] Gaonkar, G. H.; Peters, David A., "Effectiveness of Current Dynamic Inflow Models in Hover and Forward Flight", JAHS Vol. 31, No. 2, 1986.

[18] Chen, Robert T.N.; Hindson, William S., "Influences of Dynamic Inflow on the Helicopter Vertical Response”, NASA T.M. 88327, June 1986.

[19] Zhao, Xin; Curtise, H. C. Jr., "A Study of Helicopter Stability and Control Including Blade Dynamics", NASA CR 183245, Oct. 1988.

[20] Wiesner, Wayne; Kohler, Gary, "Tail Rotor Performance in Presence of Main Rotor, Ground, and Winds", Proceedings of the 29th Annual Forum of the American Helicopter Society, May 1973.

'Jourad of the American Helicopter Society 
[21] Lynn, R. R.; Robinson, F. D.; Batra, N. N.; Duhon, J. M., "Tail Rotor Design Part I-Aerodynamics", Proceedings of the 25th Annual Forum of the American Helicopter Society, May 1969.

[22] Talbot, Peter D.; Tinling, Bruce E.; Decker William A.; Chen, Robert T. N., "A Mathematical Model of a Single Main Rotor Helicopter for Piloted Simulation". NASA T.M. 84281, Sept. 1982.

[23] Chen, Robert T. N., “A Simplified Rotor System Mathematical Model for Piloted Flight Dynamics Simulation”, NASA T.M. 78575, May 1979.

[24] Prouty, Raymond W., Helicopter Performance, Stability and Control, Boston: P.W.S. Publishers, 1986.

[25] Bramwell, A. R. S., Helicopter Dynamics, London: Arnold, 1976.

[26] Heffley, Robert K.; Mnich, Marc A., "Minimum-complexity Helicopter Simulation Math Model", NASA CR 177476, April 1988.

[27] McLarty, Tyce. T., "Rotorcraft Flight Simulation with Coupled Rotor Aeroelastic Stability Analysis Volume I: Engineer's Manualn", USAAMRDL Technical Report 7641 A, May 1977.

[28] Nikolsky, Alexander A., Helicopter Analysis, New York: John Wiley \& Sons Inc., 1951.

[29] Johnson, Wayne, Helicopter Theory, Princeton Univ. Press, 1980.

[30] Pitt, Dale M.; Peters, David A., "Theoretical Prediction of Dynamic-Inflow Derivatives", Vertica Vol. 5, pp. 21 to 34, 1981.

[31] Carpenter, P.J.; Fridovich, B., "Effect of Rapid-Pitch Increase on the Thrust and Induced-Velocity Response of a Full-Scale Helicopter Rotorn, NACA TN $3044,1953$.

[32] Jewel, Joseph W. Jr.; Heyson, Harry H., "Charts of the Induced Velocity Near a Lifting Rotor", NASA MEMO 4-15-59L, May 1959.

[33] Heyson, Harry H.; Katzoff, S., "Induced Velocities Near a Lifting Rotor with Nonuniform Disk Loading",NACA Rept. 1319, 1957. 
[34] Critzos, Chris C.; Heyson, Harry H.:Boswinkle, Robert W. Jr., “Aerodynamic Characteristics of NACA 0012 Airfoil Section at Angles of Attack from $0^{\circ}$ to $180^{\circ}$. N.ICA T.N 3361, Jan. 1995.

[35] Davis, J. M.; Bennete, R. C.; Blankenship, B. L., "Rotorcraft Flight Simulation with Aeroelastic Rotor and Improved Aerodynamic representation" USAAMRDL-TR-it10A, Forth Eustis, Va., June 1974.

[36] Press, William H., Numerical Recipes in Fortran, the Art of Scientific Computing Ind ed., Cambridge, England, U. P. 1992.

[37] Gilbert, E. G., "Dynamic Error Analysis of Digital and Combined Analog-Digital Computer Systems”, SIMULATION vol. 6 No. 4, April 1966, pp. 241-257.

[38] Howe, R.M.; Fogarty, L.E., "Computer Considerations for Real Time Simulation of A Generalized Rotor Model”, NASA CR-2877, Sept. 1977.

[39] Tischler, Mark B.; Leung, Joseph G.M.; Dugan, Daniel C., "Frequency-Domain Identification of XU-15 Tilt-Rotor Aircraft Dynamics in Hovering Flight”, JAHS Vol. 30, No. 2, 1985.

[40] Bendat, Julius S.; Piersol, Allan G., Engineering Applications of Correlation And Spectral Analysis, 2nd ed., John Wiley \& Sons Inc., Toronto, 199j.

[41] Bendat, Julius S.; Piersol, Allan G., Random Data, Analysis and Measurement Procedures 2nd ed., John Wiley \& Sons Inc., Toronto, 1986.

[42] Marple, S. L. Jr. Digital Spectral Analysis with Applications, Prentice-Hall, Englewood Cliff, New Jersey, 1987.

[43] Krauss, Thomas P.;Shure, Loren; Little, John N., Signal Processing Toolbox Users' Guide for Use with Matlab, The Math Works Inc., 1994.

[44] Baskin, V. E.;Vil'dgrude, L. S.; Vozhdayer, Ye. S.; Maykaper, G. I., "Theory of the Lifting Airscrew”, NASA TT F-823, Feb. 1976.

[45] Levi, E. C., "Complex-Curve Fitting", IRE Trans. on Automatic Control, Vol. AC-4, pp. 37.44, 1959. 
BIBLIOGRAPHY

153

[16] Mope, Andreas, "Validation of a Mathematical Model for Piloted Simulation of a Bell 205 Helicopter ” MSt. Dissertation 1989, University of Toronto, Canada.

[47] Reedy, K. R. "Effect of Rotor Wake Geometry Variation on Hover Induced Power Estimation for a UH-1H Iroquois Helicopter" Aerodynamics Tech. Memorandum 384, Oct. 1986.

[48] Williams, M. J.; Arney, A. M., Helicopter Hover Performance Estimation Comparison with UH-1H Iroquois Flight Data Aerodynamic Tech. Memorandum 377. 

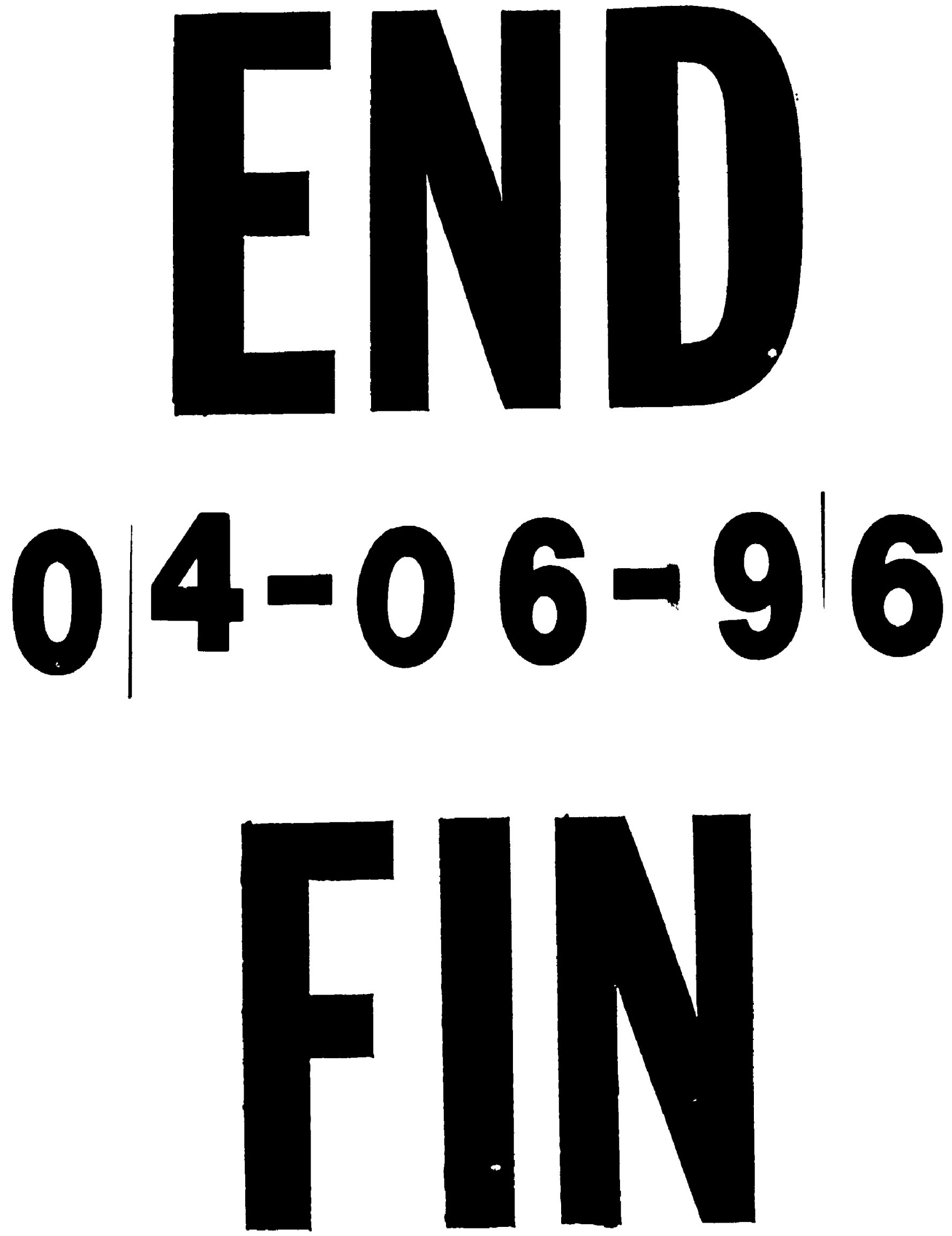